Quantitative and ecological aspects of $\underset{\text { population heterogeneity }}{\text { Listeria monocytogenes }}$

Karin Metselaar 

Quantitative and ecological aspects of Listeria monocytogenes population heterogeneity 


\section{Thesis committee}

\section{Promotors}

Prof. Dr Marcel H. Zwietering

Professor of Food Microbiology

Wageningen University

Prof. Dr Tjakko Abee

Personal chair at the Laboratory of Food Microbiology

Wageningen University

\section{Co-promotor}

Dr Heidy M.W. den Besten

Assistant professor, Laboratory of Food Microbiology

Wageningen University

\section{Other members}

Prof. Dr Michiel Kleerebezem, Wageningen University

Prof. Dr Arie H. Havelaar, University of Florida, Gainesville, United States of America

Dr Kostas Koutsoumanis, Aristotle University of Thessaloniki, Greece

Dr J an-Willem Sanders, Unilever R\&D Vlaardingen B.V.

This research was conducted under the auspices of the Graduate School VLAG (Advanced studies in Food Technology, Agrobiotechnology, Nutrition and Health Sciences) 


\title{
Quantitative and ecological aspects of Listeria monocytogenes population heterogeneity
}

\author{
Karin I. Metselaar
}

\section{Thesis}

submitted in fulfilment of the requirements for the degree of doctor at Wageningen University

by the authority of the Rector Magnificus

Prof. Dr A.P.J. Mol,

in the presence of the

Thesis Committee appointed by the Academic Board

to be defended in public

on Wednesday 25 May 2016

at 4 p.m. in the Aula. 
Karin I. Metselaar

Quantitative and ecological aspects of Listeria monocytogenes population heterogeneity

174 pages.

$\mathrm{PhD}$ thesis, Wageningen University, Wageningen, NL (2016)

With references, with summaries in English and Dutch

ISBN 978-94-6257-766-4 


\section{Table of Contents}

$\begin{array}{ll}\text { Chapter } 1 & 7\end{array}$

General introduction and outline of the thesis

Chapter 2

Isolation and quantification of highly acid resistant variants

of Listeria monocytogenes

\section{Chapter 3}

Diversity of acid stress resistant variants of Listeria monocytogenes and the potential role of ribosomal protein S21 encoded by rpsU

\section{Chapter 4}

Performance of stress resistant variants of Listeria monocytogenes in mixed species biofilms with Lactobacillus plantarum

Chapter 5

Modeling and validation of ecological behaviour of

Listeria monocytogenes wild type and stress resistant variants

Chapter 6

General discussion, conclusions and future perspectives

Summary

Acknowledgements

About the author

List of publications 


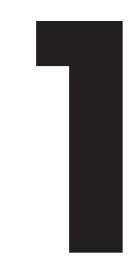

General introduction and outline of the thesis 


\section{Listeria monocytogenes: from soil organism to human pathogen}

Bacterial survival in many different environments requires a good strategy and the ability to rapidly adapt. Listeria monocytogenes is such a bacterium which is capable of thriving in many different environments [1]. L. monocytogenes can be considered a soil organism, a human pathogen and an inhabitant of foods and food-processing settings. In these different niches, many types of stress can be encountered [2] which includeamongst others thelimited availability of nutrientsin the soil, heat treatments during food processing, low temperature during storage of food products and the low $\mathrm{pH}$ of the stomach [1]. During its transmission from soil to the human host, L. monocytogenes has to cope with a range of stresses and indeed, L. monocytogenes is one of the most robust non-spore forming foodborne pathogens. This ubiquitous pathogen can grow under a wide range of temperatures, from $\sim 0^{\circ} \mathrm{C}$ to $45^{\circ} \mathrm{C}$ and it can tolerate high salt concentrations up to $12 \%(\mathrm{w} / \mathrm{v})$. The minimum $\mathrm{pH}$ required for growth is generally around $\mathrm{pH} 4.3$ [3]. This wide range of environmental conditions under which L. monocytogenes can grow and survive make it a difficult pathogen to eliminate from food processing environments. Notably, L. monocytogenes is in the top three of agents causing death due to foodborne disease [4], which highlights the importance of effective measures for L. monocytogenes control. A lot of scientific research has focused on understanding the mechanisms behind the ability of L. monocytogenes to survive, grow and adapt under suboptimal conditions as better understanding of foodborne pathogens, including their behaviour and natural habitats will lead to better control measures in food production.

\section{Ecological niches}

Due to its robustness and adaptive behaviour, L. monocytogenes can be present in different ecological niches. The transmission cycle of L. monocytogenes and the different niches encountered during this transmission cycle are displayed in Figure 1.1. L. monocytogenes is ubiquitously present in the environment, and soil was shown to be an important niche [5-7]. The incidence of L. monocytogenes in soil is relatively high and was reported to be between 8 and $44 \%$ of analysed soil samples [7]. The concentration on the other hand is relatively low and in most cases reported to be in the order of magnitude of $1-100 \mathrm{cfu} / \mathrm{g}$ in positive samples [8]. Soil types and characteristics can be very different between geographical regions and these characteristics can have a strong effect on L. monocytogenes survival [9].

Soil pH seems to be an important determinant for L. monocytogenes survival as well. Survival for more than 32 days in soils of $\mathrm{pH}$ of 6.5 and 6.9 was observed, while it did not persist for more than 6 days in a forest soil characterized by a low pH of 
5.2 [5].The temperature in soil can vary, depending on the geographical location and the season. Higher survival of L. monocytogenes was observed at lower temperature [6], although at higher altitude, and thus lower temperature, the incidence was lower than at lower altitudes, most likely related to less human and animal contact in these higher areas [9]. Animals are another reservoir for L. monocytogenes and the bacterium has been frequently isolated from livestock, with a higher incidence in cattle than in sheep and pigs [5]. Animal products like raw milk, can be a direct source of human contamination, but are also an important transmission vehicle towards food processing environments. From either soil directly or from animal faeces, plant produce can get contaminated by L. monocytogenes as well, which is another important vehicle, as well as a transmission route towards food processing environments [10]. Although fresh produce and animal products can be a cause of L. monocytogenes infection in humans, infections mostly occur through processed food products like deli meats [11, 12]. Once present in food processing environments, L. monocytogenes can be a difficult pathogen to eliminate and persistent presence in food processing facilities is repeatedly reported [13-15].

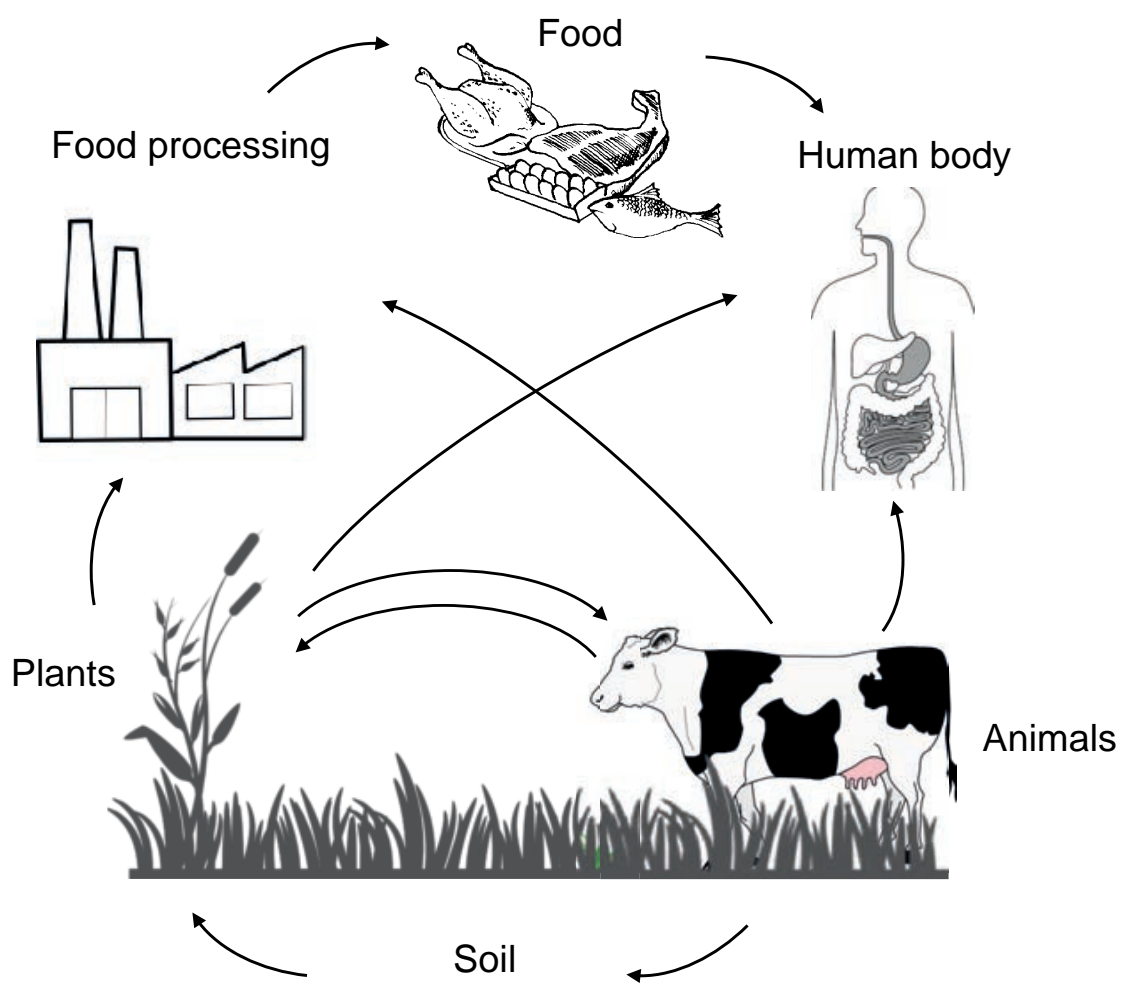

Figure 1. 1: Schematic representation of the transmission cycle and different ecological niches of Listeria monocytogenes. 


\section{Biofilm formation}

When present in natural or food processing environments, L. monocytogenes can switch to a biofilm life cycle [16]. A biofilm is a microbiologically derived sessile community characterized by cells that are irreversibly attached to a surface or to each other, are embedded in a matrix of extracellular polymeric substances and exhibit an altered phenotype with respect to growth rate and gene expression [16]. Biofilms are a challenge for the food industry, because they are difficult to remove from processing lines. Bacteria within biofilms show increased resistance towards cleaning and disinfection strategies compared to their planktonic counterparts $[13,17]$. There are several potential causes for the increased resistance of biofilms. One of them can be attributed to the protected environment at the inner layers of the biofilms. Also, cells embedded in a biofilm were shown to grow slower than in planktonic cultures and reduced growth rate has been implicated with increased resistance to environmental stresses [16]. The ability to form biofilms on surfaces is dependent on several environmental conditions. Temperature was shown to be an important factor in initial adherence to surfaces, with a higher adherence potential at higher temperatures [18-20]. Also nutrient availability, $\mathrm{pH}$ and the presence of other microflora are important determinants in biofilm formation [17, 20, 21]. Heterogeneity exists within biofilms, because the conditions at different locations in the biofilm are different. Oxygen and nutrient availability are variable depending on the age and location of the biofilm and this leads to differences in gene expression $[22,23]$. Also in biofilms, the potential to adapt to different environments assists in the survival and persistent presence of L. monocytogenes in food processing environments. Biofilms are thought to be a major source of contamination in food processing facilities, mostly as a result of detachment of cells from biofilms which end up in food products $[13,23,24]$. The contamination of products with L. monocytogenes is often associated with contaminated equipment or environments during processing and especially when the product supports growth of L. monocytogenes, this might be a potential food safety issue [17]. This is highlighted by for example the large listeriosis outbreak that occurred in Canada in 2008, which was attributed to sliced deli meats [25]. Contaminated mechanical meat slicers were identified as the most likely source of L. monocytogenes contamination of the deli meats [26]. L. monocytogenes was already found to be present in the production facilities in the weeks prior to the outbreak but sanitation procedures used prior to the outbreak were ineffective. Outbreaks like the one reported in Canada in 2008, which was responsible for 57 people ill and 22 deaths, highlights the importance of effective measures to control L. monocytogenes in food processing environments. 


\section{Adaptation to niches}

Apart from its robustness and ability to form biofilms, L. monocytogenes has great potential to adapt to rapidly changing environments. This ability is mostly due to the presence of several sigma factors [27]. Sigma factors are subunits of RNA polymerase which are responsible for recognition of the promotor region for a certain gene and for transcription initiation of that gene. By transcription initiation, sigma factors enable expression of new sets of genes under changing environmental conditions [2]. Alternative sigma factors, like $\sigma^{\mathrm{B}}$, are inactive when the cells are not stressed, but when a stressful environment is encountered, rapid activation of the alternative sigma factor and thus gene expression involved in general or specific stress response is initiated. $\sigma^{\mathrm{B}}$ has been shown to be involved in the response to different types of stress that can be encountered outside the host as well as inside the host [28-30]. $\sigma^{\mathrm{B}}$ can be considered a major factor in the ability of L. monocytogenes to withstand different harsh conditions and it can therefore contribute to its ubiquitous presence in different environmental niches. The ability to adapt to different niches and the problems this can cause is highlighted by several outbreaks in the last decades.

\section{Foodborne pathogen}

Most of the human L. monocytogenes infections are foodborne [13]. Upon ingestion, L. monocytogenes switches from soil bacterium to human pathogen and a whole range of other stresses is encountered before successful invasion. Throughout this infection route, a number of hurdles have to be overcome [31, 32]. The low $\mathrm{pH}$ in the stomach is the first hurdle and has the purpose of keeping harmful bacteria outside. The $\mathrm{pH}$ of the gastric fluid within the stomach is usually around $\mathrm{pH} 1.5$, but can increase to between $\mathrm{pH} 3$ and 5 after eating [33]. After stomach passage, L. monocytogenes enters the blood stream via the intestinal epithelium and subsequently moves to the liver and spleen. Whereas the healthy part of the human population mostly has to deal with flu-like symptoms or self-limiting gastrointestinal complaints upon L. monocytogenes infection, listeriosis is a significant health risk for new-born infants, elderly people, immunocompromised people and pregnant women and their foetus [12, 34]. Although the number of L. monocytogenes outbreaks and sporadic cases are relatively low, the impact of such outbreaks is very significant with a case-fatality rate usually as high as 20-30\% [12]. L. monocytogenes is therefore amongst the foodborne pathogens causing most deaths annually $[35,36]$ and the highest number of disability adjusted life years per case [37]. Examples of recent outbreaks are the Canada outbreak discussed above, and a large L. monocytogenes outbreak in the US in 2011 which was attributed to contaminated cantaloupes. The contamination source in this outbreak was most likely a combination of unsuitable equipment and pour hygienic practices during cleaning and storage of the whole cantaloupes. This outbreak resulted in 147 
people ill and 22 deaths [38]. The high burden of disease, high mortality rate and the high cost of illness of around $\$ 2.6$ billion in the US in 2012 [39] definitely make L. monocytogenes a pathogen of major concern and the topic of research for many years since its recognition as foodborne pathogen in the 1980s [40].

\section{Population heterogeneity}

\section{Non-linear inactivation}

Due to the serious nature of listeriosis the regulations for L. monocytogenes in food products are very strict and L. monocytogenes must be absent in 25 grams in products that support growth of L. monocytogenes ( 5 samples) or are intended for infants and special medical purposes (10 samples) [41]. As mentioned above, L. monocytogenes can be present in raw materials or in food processing environments and therefore most food products receive a processing step to inactivate not only L. monocytogenes but also other pathogens and spoilage organisms. When all individuals in a bacterial population behave identical, the survival curve upon a processing step will follow first order kinetics, resulting in a linear inactivation curve when the number of survivors is plotted on a logarithmic scale against time [42]. In practice, linear inactivation curves are the exception rather than the rule and often, the presence of a shoulder and/ or a tail is observed [43]. The presence of a shoulder can indicate that some time is needed before the cells are affected by the stress and is more often observed upon exposure to mild stress. Shoulders can also be caused by clumping of the cells and with more severe stress exposure, the shoulder length generally decreases [42]. The presence of a tail indicates that a part of the population is more resistant to the stress than the main population. Significant tailing upon inactivation treatment has also been reported for multiple types of stress, including acid and high hydrostatic pressure inactivation [44, 45] and for several microorganisms [46]. An example of tailing is shown in Figure 1.2

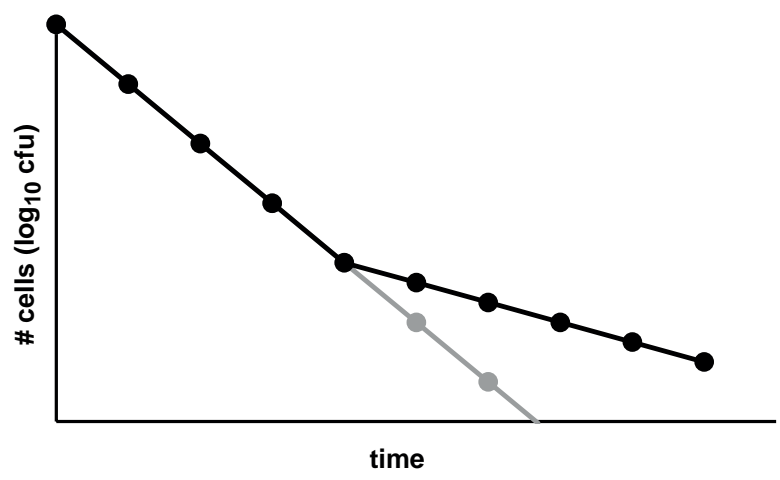

Figure 1.2: Schematic representation of tailing of an inactivation curve (black line) versus linear inactivation (grey line) upon stress exposure of a bacterial population. 


\section{Heterogeneity in stress response}

The presence of tailing indicates a heterogeneous stress response and the presence of different phenotypes. Generating variable phenotypes may be beneficial for the survival of the population under adverse conditions, although in many cases it is a process that costs a lot of energy. Heterogeneity might not be ideal for bacteria under homogenous conditions but mathematical studies support the idea that in a variable environment a heterogeneous population can outcompete a homogeneous population [47]. A heterogeneous stress response of a bacterial population can have several causes and can roughly be divided in phenotypic and genotypic heterogeneity. Generally, upon transfer to a stressful environment, bacteria activate adaptive stress networks, leading to transcription, translation and activation of stress-related cellular components. Heterogeneity in the expression of these stress regulatory genes, which are not caused by mutations, are considered to underlie phenotypic heterogeneity and can have several causes [48]. Bistability is one such example and occurs in populations of genetically identical cells, grown in homogeneous and identical environments and is considered a stochastic process [49]. Bistability is the phenomenon in which expression of certain genes is high in one part of the population and low in another part of the population. This difference is caused by random stochastic fluctuations in biochemical reactions in the cell. At the population level this results in two different phenotypes within the population [47]. Another strategy that results in a phenotypically heterogeneous stress response is bethedging [47]. Bet-hedging will increase the overall fitness of the population because some offspring will have the correct adaptation for any given situation. An example of bet-hedging is the presence of cells in a dormant, non-dividing state which confers increased stress resistance, also referred to as bacterial persisters. This phenotype is classified as a transient characteristic [50], which is expressed by almost all bacterial species and was recently also confirmed in L. monocytogenes [51]. Mechanisms of bacterial persister formation are not well understood as they are small in numbers and transient and can change with the type of stress and environment. The switch from normal growth to persistence and vice versa is stochastic and epigenetic in nature [52]. Recent findings have shown that a variety of environmental conditions can induce the formation of persister subpopulations, including starvation, carbon source transitions, DNA damage-induced SOS response, and exposure to antibiotics [53, 54]. Because of their dormant, stress resistant phenotype, bacterial persisters are likely objects to become domestic flora in food production lines or to end up in biofilms. When the same strain is isolated from the same niche two or more times with some time in between, this is also referred to as persistence. However, their reoccurrence is not necessarily caused by bet-hedging, which makes the term 'persisters' confusing. Persistent presence of strains in natural or food production niches can also be caused by other types of heterogeneity or strain characteristics. 


\section{Stable stress resistant variants}

Another cause for tailing of inactivation curves can be the presence of stable stress resistant variants. The difference between tailing caused by physiological heterogeneity and the presence of stable resistant variants is that physiological characteristics are transient; when survivors of the tail are sub-cultured and exposed to stress again, they will show an equal sensitivity as the main population. Stable stress resistant variants on the other hand harbour a mutation which makes them more stress resistant than the main population. Although both transient and stable resistance may be relevant in food processing, stable resistant variants are the main topic of the work in this thesis. The difference between tailing caused by phenotypic and genotypic heterogeneity is illustrated in Figure 1.3.
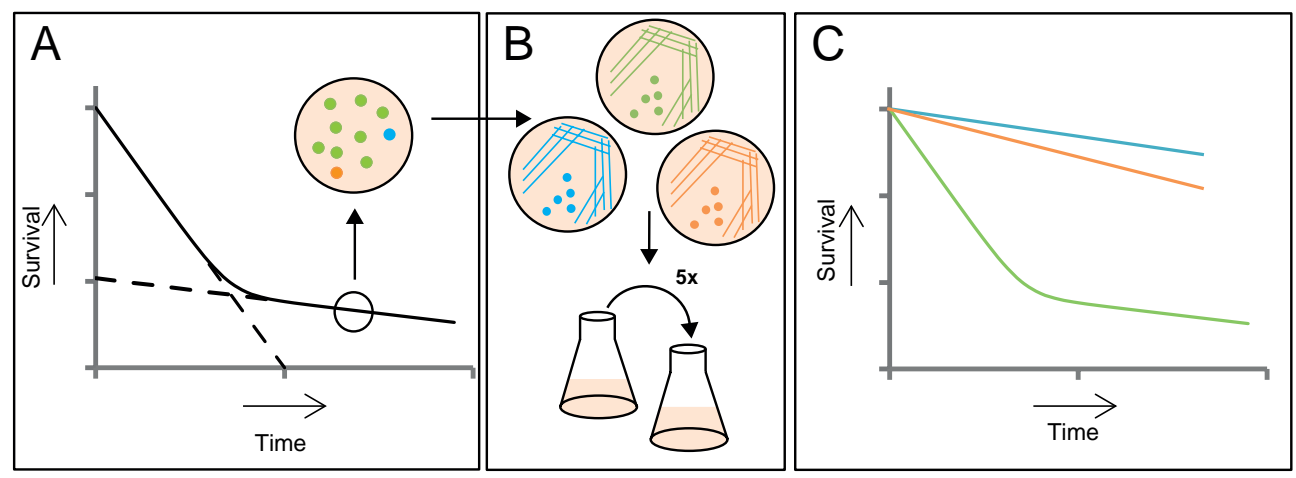

Figure 1.3: Schematic presentation of genotypic and phenotypic heterogeneity. (A) Tailing is observed upon stress exposure. The population in the tail consists of both phenotypically more resistant cells (green colonies) and stable resistant variants (blue and orange colonies) (A). When colonies are randomly selected and inoculated in fresh medium followed by repeated propagation providing a genetically stable culture (B) and tested on stress resistance again, the phenotypically resistant isolates (green) show the same kinetic as the initial WT and the stable resistant variants show increased stress resistance (C). Adapted from Abee et al. [55].

The presence of a stable stress resistant variant has been established and described in detail for L. monocytogenes exposed to high hydrostatic pressure (HHP). Karatzas and Bennik [56] were the first to isolate a pressure tolerant variant of L. monocytogenes Scott A after a single exposure of a mid-exponential phase culture to $400 \mathrm{MPa}$ for 20 minutes. Besides an increased tolerance towards HHP, the variant was characterized by reduced growth rate over a temperature range of $8-30^{\circ} \mathrm{C}$, lower final $\mathrm{OD}_{600}$, the variant was immotile and the cells were about twice as long as the WT cells. The variant showed increased resistance to heat, acid and hydrogen peroxide as well. Later it was found that the increased resistance of this variant was due to a deletion of 3 basepairs in the glycine repeat region of ctsR, resulting in a single amino acid deletion [57]. ctsR encodes a Class III heat shock repressor, which negatively regulates the clp heat shock genes. The observed deletion in ctsR resulted 
in a defect in the repressor function of CtsR with a constant activation of the Clp proteases and increased stress resistance as a result. It was shown that this type of stable stress resistant variant was not a single case for this specific strain, as later more ctsR variants of different L. monocytogenes strains were isolated upon HHP exposure [45, 58, 59]. Van Boeijen et al. [60] showed that also heat exposure leads to selection for stable stress resistant variants with a mutation in ctsR. However, not all the isolated heat and HHP resistant variants had a mutation in their ctsR gene, indicating other underlying mechanisms that still have to be unravelled. An extensive phenotypic characterization of a set of 24 HHP resistant variants of L. monocytogenes showed a large phenotypic diversity amongst the variants [59]. In a phenotypic cluster analysis the ctsR variants clustered together. Another large cluster was made up of immotile variants. Virulence of the variants was evaluated as well [61]. The ctsR variants showed reduced virulence potential in a mouse model, but some other variants, with an unknown mutation, showed similar virulenceas the WT in mouse spleen and liver. Combined with increased heat and acid resistance, these types of variants are a potential food safety risk. The work that has been performed on stable stress resistant variants in the past decade showed that a large diversity exists within the L. monocytogenes population. This diversity potentially allows for growth and survival under a wide range of environmental conditions and can therefore be considered a strategy to thrive in different ecological niches that can be encountered by the population. However, many aspects regarding the mechanisms behind the origin of the variants and the actual impact on food safety still has to be unravelled.

\section{Microbial inactivation in food preservation}

\section{Mild preservation}

When a bacterial population encounters a stringent enough treatment with the aim to inactivate the population, the presence of heterogeneity, either with a physiological or genetic background, is not of major concern. During the past decade consumers have been increasingly concerned about the processing or treatment history of food products. The industry has seen an increased demand for food produced with limited use of chemical preservation. The demand for fresh food with a long shelf life presents considerable challenges to the industry regarding prevention of contamination and growth of human pathogens in their products [17, 62]. To answer to the demand for minimally processed foods, the trend in preservation has shifted to milder heat treatments and new preservation methods like HHP and pulsed electric field (PEF). These milder treatments lead to improved product quality, 
maintenance of nutritional value and better sensory properties. When applying these mild preservation techniques, the presence of small, resistant subpopulations, with a higher survival rate than the main population, might become a concern with respect to food safety. Especially the use of HHP to ensure food safety has been shown to induce tailing, suggesting a small portion of the population to be relatively resistant to the applied pressure. This phenomenon seems to be general as it was observed for several microorganisms (e.g. Salmonella, Escherichia coli, L. monocytogenes). Thus, the trend towards mild preservation highlights the importance to gain more knowledge about the presence and impact of population heterogeneity in L. monocytogenes.

\section{Acid as food preservative}

Acid is an important hurdle for bacteria to overcome, both in food products as well as during gastric passage and in the macrophage phagosome [33]. Acid resistance can therefore be considered an important niche factor [63]. L. monocytogenes developed different strategies to deal with acidic environments. Acid resistance is affected by the growth phase, history of the cells and the environmental conditions [64]. Stationary phase cells are more acid resistant than exponential phase cells. Growth under or exposure to mild, non-lethal $\mathrm{pH}$ stress confers protection to subsequent exposure to lethal $\mathrm{pH}$, a phenomenon known as the acid tolerance response (ATR) [65]. The acid resistance of an organism is determined by the combination of inducible strategies to remove protons, alkalinize the environment, change the composition of the cell membrane and produce general stress proteins. The most important and most wellstudied system in L. monocytogenes is the glutamate decarboxylase (GAD) system [66]. An extracellular glutamate is imported by an antiporter in exchange for an intracellular $\gamma$-aminobutyrate (GABA). Each molecule of glutamate is decarboxylated by a decarboxylase to produce a molecule of GABA. During this process a proton is consumed. This results in an increase of the cytoplasmic $\mathrm{pH}$ and thus protects the cell against the acidic environmental conditions [67]. Recently, a new model for the GAD system has been proposed by Karatzas et al. [68], in which two different GAD systems were discriminated. The intracellular GAD system uses metabolically synthesized glutamate and the extracellular system uses environmental glutamate which is accumulated in the cell by dedicated transporters. The GAD system is visualized in Figure 1.4.

Sigma factor B $\left(\sigma^{B}\right)$ plays a major role in the general stress response of $\mathrm{L}$. monocytogenes and it also plays a role in acid stress resistance. $\sigma^{\mathrm{B}}$ regulates the expression of several stress proteins with protein repair and chaperone activity functions, such as DnaK, GroEL, HtrA and the Clp proteases [27]. $\sigma^{\mathrm{B}}$ plays a role in acid resistance through different pathways. Firstly, it has a role in the ATR, as 
it was shown that a $\sigma^{\mathrm{B}}$ deletion mutant showed a lower degree of ATR than the WT strain. Also, the GAD system as described above is reported to be at least partially $\sigma^{\mathrm{B}}$ regulated [30].

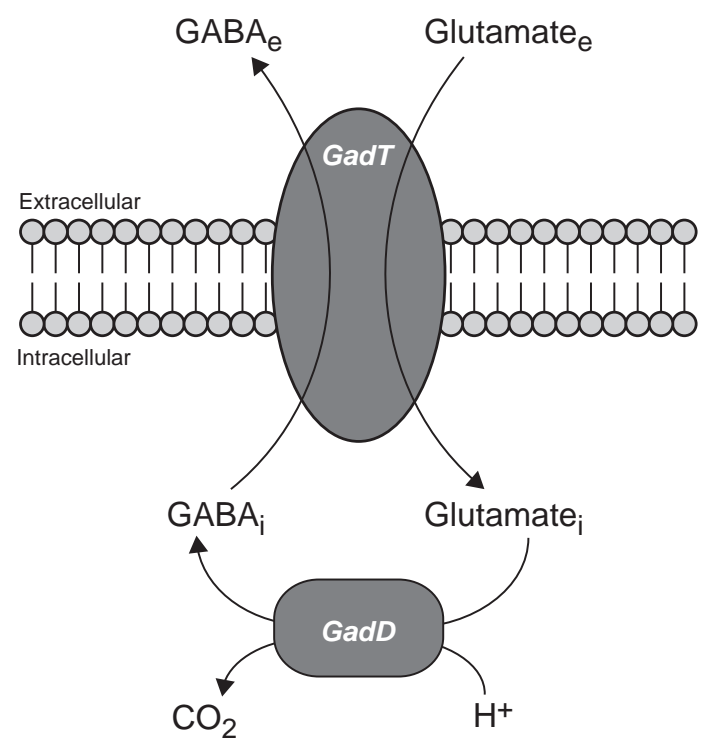

Figure 1.4: Model of the glutamate decarboxylase system in L. monocytogenes as proposed by Karatzas et al. Adapted from [68].

\section{Predictive microbiology}

Predictive microbiology, or quantitative microbiology, is a growing discipline within food microbiology and aims to develop and use mathematical and statistical models to describe microbial behaviour. Modelling can be used to quantitatively describe growth or inactivation kinetics but also to predict microbial behaviour under certain conditions [70]. The benefit of predictive microbiology is that, when done appropriately, it can reduce the amount of labour intensive and costly challenge tests. An assumption when using predictive models is that biological responses to environmental factors are reproducible. It is important that predictive models are validated, as microbial heterogeneity, interaction between different environmental factors or inaccuracy in measuring the environmental factors may lead to systematic errors in predictions. Also several variability factors (e.g. strain, biological and experimental variability) should be taken into account. Therefore, the outcome of predictive models should be considered an indication rather than an absolute value. A wealth of microbial growth and inactivation models have been developed in the recent years and the choice of the appropriate model is critical when describing and predicting behaviour. The model with the smallest number of parameters that 
adequately describe the data is preferred over a more complex model [71, 72]. Another criterion is that models with parameters that have a biological meaning are preferred over models with mathematical parameters since this makes interpretation of the parameters easier. Various models describing linear and also non-linear inactivation kinetics are available in literature. Table 1.1 shows models that are able to describe different inactivation patterns and range from the most simple first-order model to the more complex models that are able to describe biphasic inactivation with a shoulder.

Predictive models are often used in Quantitative Microbial Risk Assessment (QMRA). QMRA is a process which is used as a tool to evaluate the risk associated with the consumption of a food product [79]. QMRA consists of four steps in which the severity of a hazard is combined with the prevalence and the concentration of this hazard: hazard identification, hazard characterization, exposure assessment, and risk characterization [8o, 81]. The presence of population diversity can affect the outcome of risk assessments as it may have implications for stress resistance or virulence. Variability is one of the major challenges in the field of predictive microbiology. Variability can be caused by experimental variation like pipetting errors, biological variation caused by the use of different cultures or strain variability. But also the presence of heterogeneity within a population makes it difficult to predict the behaviour under different environmental conditions. Better quantitative knowledge on population heterogeneity will allow for incorporation of heterogeneity in predictive models and risk assessments which will lead to more realistic predictions. 


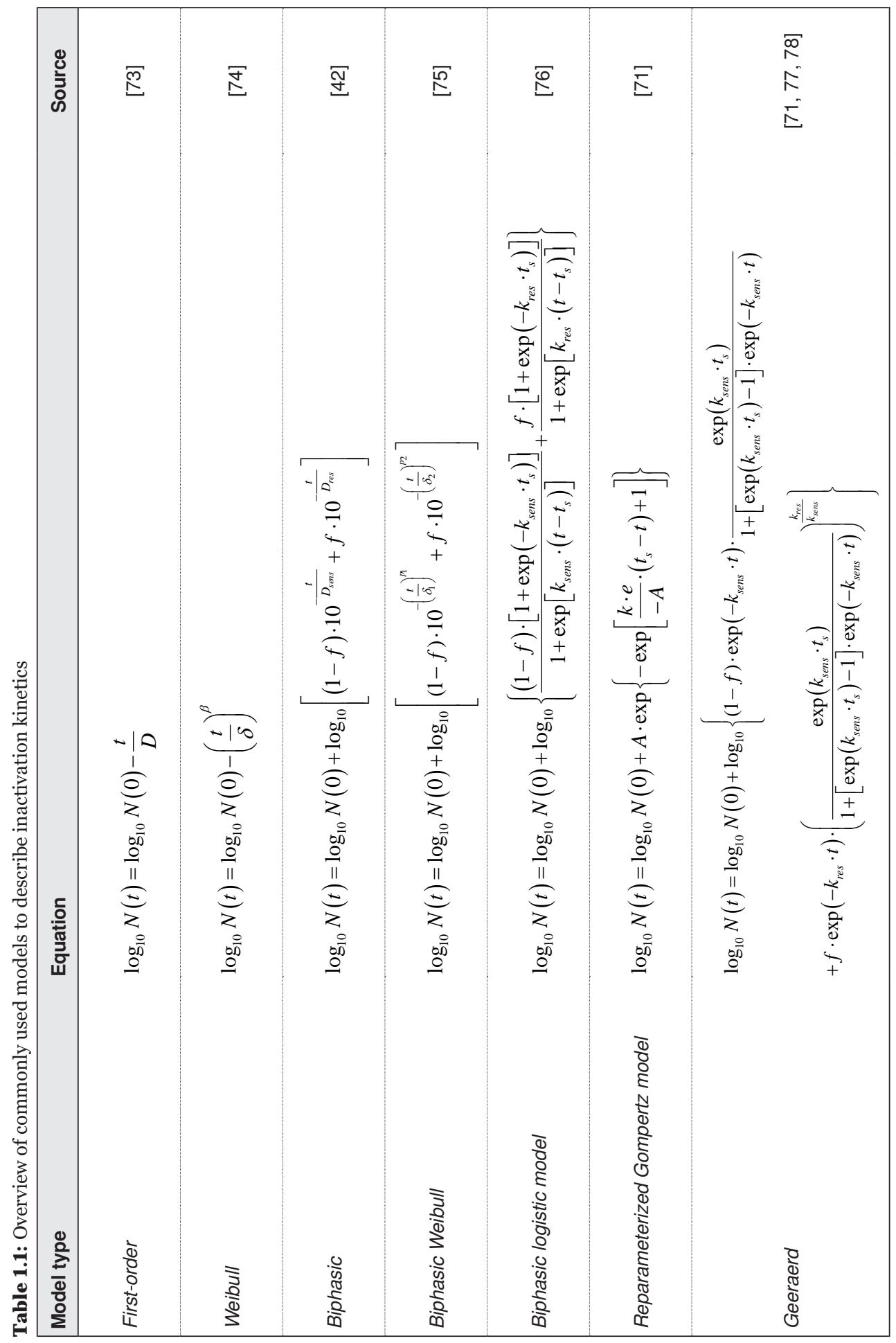




\section{Aim and outline of the thesis}

The tailing of L. monocytogenes stress-induced inactivation curves and conceivable roles of stress resistant variants has been well established and qualitative data is available for a number of variants isolated upon HHP and heat exposure. The ecological behaviour and the potential impact of these variants on food safety are not studied in detail yet. In order to evaluate this, more knowledge is needed on the conditions that may lead to selection for stress resistant variants. Also, more insights in the mechanisms underlying the increased resistance of the variants may help to understand their behaviour. And lastly, more quantitative knowledge is needed to incorporate the behaviour of the stress resistant variants into predictive models and risk assessments. The objective of this research was to evaluate if $\mathrm{L}$. monocytogenes population diversity and the presence of stable resistant variants is a general phenomenon that is observed upon different types of stress exposure and to evaluate the ecological behaviour of these stable resistant variants. The approach followed to reach the objective was to get more qualitative, quantitative and mechanistic knowledge on the behaviour of stable stress resistant variants and to extent the knowledge to another type of stress, namely acid stress. Acid stress was chosen as it is an important hurdle both in food preservation, as well as in stomach survival. Figure 1.5 displays an overview of the different topics addressed in this thesis. In Chapter 2, the non-linear inactivation kinetics of L. monocytogenes upon acid exposure were quantitatively described. A commonly used biphasic inactivation model was reparameterized, which improved the statistical performance of the model and resulted in more accurate estimation of the resistant fraction within L. monocytogenes WT populations. The observed tailing suggested that stable stress resistant variants might also be found upon acid exposure. Evaluation of the population in the tail indeed resulted in theisolation of stable acid resistant variants.

In Chapter 3, these variants were further characterized phenotypically and cluster analysis was performed. Whole genome sequencing of a set of variants was performed and a new potential target gene responsible for the increased resistant phenotype was identified. The identified mutation was found in all variants that comprised the same phenotypic cluster. Chapter 4 and 5 focus on the ecological behaviour and potential impact of stress resistant variants on food safety. In Chapter 4 , the performance in mixed species biofilms with Lactobacillus plantarum was evaluated. It was hypothesized that the acid resistant variants might also show better survival in biofilms with L. plantarum, which provide an acidic environment by lactose fermentation. Increased acid resistance turned out not to be directly related to increased survival, although some variants show significantly better survival than the WT or other variants. Increased performance in biofilm mode may be a risk 
factor for persistent presence and thus for food safety. In Chapter 5, quantitative data on survival and robustness was obtained for the WT and variants, which was subsequently used to predict the behaviour of WT and variants under different environmental conditions and along a model food chain. This gave more insight in the trade-off between increased stress resistance and growth capacity and the potential impact of this trade-off on food safety. Finally, in Chapter 6 the results of the work in this thesis are combined, the relevance and impact are discussed and recommendations for future research are presented. Overall, the work presented in this thesis provided more insight in the mechanisms underlying increased resistance of stress resistant variants, a better overview of the presence of stress resistant variants in populations of $\mathrm{L}$. monocytogenes and quantitative data on the behaviour of stress resistant variants which can be implemented in predictive microbiology and quantitative risk assessments.

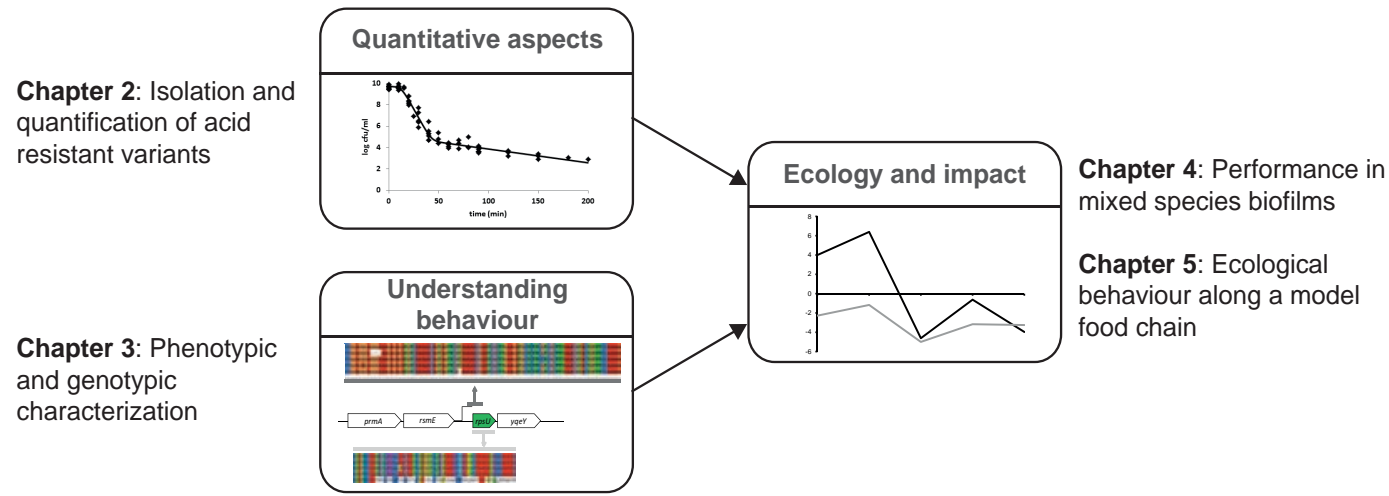

Figure 1.5: Overview of the different research topics addressed in this thesis. 


\section{References}

1. Freitag, N.E., G.C. Port, and M.D. Miner, Listeria monocytogenes - From saprophyte to intracellular pathogen. Nature Reviews Microbiology, 2009. 7(9): p. 623-628.

2. Chaturongakul, S., S. Raengpradub, M. Wiedmann, and K.J. Boor, Modulation of stress and virulence in Listeria monocytogenes. Trends in Microbiology, 2008. 16(8): p. 388-396.

3. Van der Veen, S., R. Moezelaar, T. Abee, and M.H.J. Wells-Bennik, The growth limits of a large number of Listeria monocytogenes strains at combinations of stresses show serotype and nichespecific traits. J ournal of Applied Microbiology, 2008. 105(5): p. 1246-1258.

4. CDC, CDC estimates of foodborne illness in the United States. 2011: http://www.cdc.gov/ foodborneburden/PDFs/FACTSHEET_A_FINDINGS_updated4-13.pdf.

5. Locatelli, A., A. Spor, C. J olivet, P. Piveteau, and A. Hartmann, Biotic and abiotic soil properties influence survival of Listeria monocytogenes in soil. PLoS ONE, 2013. 8(10).

6. McLaughlin, H.P., P.G. Casey, J . Cotter, C.G.M. Gahan, and C. Hill, Factors affecting survival of Listeria monocytogenes and Listeria innocua in soil samples. Archives of Microbiology, 2011. 193(11): p. 775-785.

7. Vivant, A.L., D. Garmyn, and P. Piveteau, Listeria monocytogenes, a down-to-earth pathogen. Frontiers in Cellular and Infection Microbiology. 2013 Nov 28;3:87.

8. Dowe, M.J., E.D. J ackson, J.G. Mori, and C.R. Bell, Listeria monocytogenes survival in soil and incidence in agricultural soils. J ournal of Food Protection, 1997. 60(10): p. 1201-1207.

9. Linke, K., I. Rückerl, K. Brugger, R. Karpiskova, J. Walland, S. Muri-Klinger, . . . B. Stessl, Reservoirs of Listeria species in three environmental ecosystems. Applied and Environmental Microbiology, 2014. 80(18): p. 5583-5592.

10. Weller, D., M. Wiedmann, and L.K. Strawn, Spatial and temporal factors associated with an increased prevalence of Listeria monocytogenes in spinach fields in New York State. Applied and Environmental Microbiology, 2015. 81(17): p. 6059-6069.

11. FDA, Interpretative summary: Quantitative assessment of relative risk to public health from foodborne Listeria monocytogenes among selected categories of ready-to-eat foods. 2003.

12. Lomonaco, S., D. Nucera, and V. Filipello, The evolution and epidemiology of Listeria monocytogenes in Europe and the United States. Infection, Genetics and Evolution, 2015. 35: p. 172-183.

13. Ferreira, V., M. Wiedmann, P. Teixeira, and M.J. Stasiewicz, Listeria monocytogenes persistence in food-associated environments: Epidemiology, strain characteristics, and implications for public health. J ournal of Food Protection, 2014. 77(1): p. 150-170.

14. Holch, A., K. Webb, O. Lukjancenko, D. Ussery, B.M. Rosenthal, and L. Gram, Genome sequencing identifies two nearly unchanged strains of persistent Listeria monocytogenes isolated at two different fish processing plants sampled 6 years apart. Applied and Environmental Microbiology, 2013. 79(9): p. 2944-2951.

15. Verghese, B., M. Lok, J. Wen, V. Alessandria, Y. Chen, S. Kathariou, and S. Knabel, comK prophage junction fragments as markers for Listeria monocytogenes genotypes unique to individual meat and poultry processing plants and a model for rapid niche-specific adaptation, biofilm formation, and persistence. Applied and Environmental Microbiology, 2011. 77(10): p. 3279-3292.

16. Donlan, R.M. and J.W. Costerton, Biofilms: Survival mechanisms of clinically relevant microorganisms. Clinical Microbiology Reviews, 2002. 15(2): p. 167-193.

17. Møretrø, T. and S. Langsrud, Listeria monocytogenes: biofilm formation and persistence in foodprocessing environments. Biofilms, 2004. 1(02): p. 107-121.

18. Belessi, C.E.A., A.S. Gounadaki, A.N. Psomas, and P.N. Skandamis, Efficiency of different sanitation methods on Listeria monocytogenes biofilms formed under various environmental conditions. International J ournal of Food Microbiology, 2011. 145(SUPPL. 1): p. S46-S52.

19. Kadam, S.R., H.M.W. den Besten, S. van der Veen, M.H. Zwietering, R. Moezelaar, and T. Abee, Diversity assessment of Listeria monocytogenes biofilm formation: Impact of growth condition, serotype and strain origin. International J ournal of Food Microbiology, 2013. 165(3): p. 259-264.

20. Nilsson, R.E., T. Ross, and J.P. Bowman, Variability in biofilm production by Listeria monocytogenes correlated to strain origin and grouth conditions. International Journal of Food Microbiology, 2011. 150(1): p. 14-24. 
21. Carpentier, B. and D. Chassaing, Interactions in biofilms between Listeria monocytogenes and resident microorganisms from food industry premises. International Journal of Food Microbiology, 2004. 97(2): p. 111-122.

22. Grote, J., D. Krysciak, and W.R. Streit, Phenotypic heterogeneity, a phenomenon that may explain why quorum sensing does not always result in truly homogenous cell behavior. Applied and Environmental Microbiology, 2015. 81(16): p. 5280-5289.

23. Bridier, A., P. Sanchez-Vizuete, M. Guilbaud, J.C. Piard, M. Naïtali, and R. Briandet, Biofilm-associated persistence of food-borne pathogens. Food Microbiology, 2015. 45, Part B(0): p. 167178.

24. Brooks, J.D. and S.H. Flint, Biofilms in the food industry: Problems and potential solutions. International J ournal of Food Science and Technology, 2008. 43(12): p. 2163-2176.

25. Currie, A., J.M. Farber, C. Nadon, D. Sharma, Y. Whitfield, C. Gaulin, . . . U. Sierpinska, Multi-provincelisteriosis outbreak linked to contaminated deli meat consumed primarily in institutional settings, Canada, 2008. Foodborne Pathogens and Disease, 2015. 12(8): p. 645-652.

26. Weatherill, S., Report of the independent investigator into the 2008 listeriosis outbreak. 2009, Government of Canada.

27. O'Byrne, C.P., K.A.G. Karatzas, S.S. Allen I. Laskin, and M.G. Geoffrey, Chapter 5: The Role of Sigma B in the stress adaptations of Listeria monocytogenes: Overlaps between stress adaptation and virulence, in Advances in Applied Microbiology. 2008, Academic Press. p. 115-140.

28. Abram, F., E. Starr, K.A.G. Karatzas, K. Matlawska-Wasowska, A. Boyd, M. Wiedmann, . . C.P. O'Byrne, Identification of components of the sigma B regulon in Listeria monocytogenes that contribute to acid and salt tolerance. Applied and Environmental Microbiology, 2008. 74(22): p. 68486858.

29. Chaturongakul, S., S. Raengpradub, M.E. Palmer, T.M. Bergholz, R.H. Orsi, Y. Hu, . . . K.J . Boor, Transcriptomic and phenotypic analyses identify coregulated, overlapping regulons among PrfA, CtsR, HrcA, and the alternative sigma factors $\sigma \mathrm{b}, \sigma \mathrm{c}, \sigma \mathrm{h}$, and $\sigma \mathrm{l}$ in Listeria monocytogenes. Applied and Environmental Microbiology, 2011. 77(1): p. 187-200.

30. Wemekamp-Kamphuis, H.H., J .A. Wouters, P.P.L.A. De Leeuw, T. Hain, T. Chakraborty,

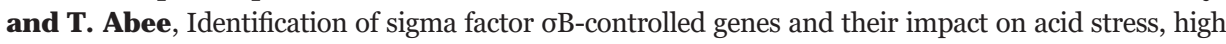
hydrostatic pressure, and freezesurvival in Listeria monocytogenes EGD-e. Applied and Environmental Microbiology, 2004. 70(6): p. 3457-3466.

31. Kathariou, S., Listeria monocytogenes virulence and pathogenicity, a food safety perspective. J ournal of Food Protection, 2002. 65(11): p. 1811-1829.

32. Vázquez-Boland, J.A., M. Kuhn, P. Berche, T. Chakraborty, G. Domínguez-Bernal, W. Goebel, . . . J. Kreft, Listeria pathogenesis and molecular virulence determinants. Clinical Microbiology Reviews, 2001. 14(3): p. 584-640.

33. Cotter, P.D. and C. Hill, Surviving the acid test: Responses of Gram-positive bacteria to low $\mathrm{pH}$. Microbiology and Molecular Biology Reviews, 2003. 67(3): p. 429-453.

34. Lyytikäinen, O., U.M. Nakari, S. Lukinmaa, E. Kela, N. Nguyen Tran Minh, and A. Siitonen, Surveillance of listeriosis in Finland during 1995-2004. Euro surveillance : bulletin européen sur les maladies transmissibles = European communicable disease bulletin., 2006. 11(6): p. 82-85.

35. Scallan, E., R.M. Hoekstra, F.J. Angulo, R.V. Tauxe, M.A. Widdowson, S.L. Roy, . . . P.M. Griffin, Foodborne illness acquired in the United States-Major pathogens. Emerging Infectious Diseases, 2011. 17(1): p. 7-15.

36. Thomas, M.K., R. Murray, L. Flockhart, K. Pintar, A. Fazil, A. Nesbitt, . . . F. Pollari, Estimates of foodborne illness-related hospitalizations and deaths in Canada for 30 specified pathogens and unspecified agents. Foodborne Pathogens and Disease, 2015. 12(10): p. 820-827.

37. Havelaar, A.H., J .A. Haagsma, M.J.J . Mangen, J .M. Kemmeren, L.P.B. Verhoef, S.M.C. Vijgen, . . . W. van Pelt, Disease burden of foodborne pathogens in the Netherlands, 2009. International J ournal of Food Microbiology, 2012. 156(3): p. 231-238.

38. McCollum, J.T., A.B. Cronquist, B.J. Silk, K.A. J ackson, K.A. O'Connor, S. Cosgrove, . . . B.E. Mahon, Multistate outbreak of listeriosis associated with cantaloupe. New England J ournal of Medicine, 2013. 369(10): p. 944-953. 
39. Hoffmann, S., M.B. Batz, and J.G. Morris Jr, Annual cost of illness and quality-adjusted life year losses in the united states due to 14 foodborne pathogens. J ournal of Food Protection, 2012. 75(7): p. 1292-1302.

40. Seeliger, H.P.R., Listeriosis - History and actual developments. Infection, 1988. 16(2 Supplement): p. S80-S84.

41. European Commission, Commission Regulation (EC) No 1441/2007 of 5 December 2007 amending Regulation (EC) No 2073/2005 on microbiological criteria for foodstuffs. 2007.

42. Cerf, O., Tailing of survival curves of bacterial spores. J ournal of Applied Microbiology, 1977. 42(1): p. 1-19.

43. van Boekel, M.A.J.S., On the use of the Weibull model to describe thermal inactivation of microbial vegetative cells. International J ournal of Food Microbiology, 2002. 74(1- 2): p. 139-159.

44. Rajkovic, A., N. Smigic, M. Uyttendaele, H. Medic, L. de Zutter, and F. Devlieghere, Resistance of Listeria monocytogenes, Escherichia coli O157:H7 and Campylobacter jejuni after exposureto repetitive cycles of mild bactericidal treatments. Food Microbiology, 2009. 26(8): p. 889-895.

45. Van Boeijen, I.K.H., R. Moezelaar, T. Abee, and M.H. Zwietering, Inactivation kinetics of three Listeria monocytogenes strains under high hydrostatic pressure. Journal of Food Protection, 2008. 71(10): p. 2007-2013.

46. Geeraerd, A.H., C.H. Herremans, and J.F. Van Impe, Structural model requirements to describe microbial inactivation during a mild heat treatment. International J ournal of Food Microbiology, 2000. 59(3): p. 185-209.

47. Veening, J.W., W.K. Smits, and O.P. Kuipers, Bistability, epigenetics, and bet-hedging in bacteria. Annual Review of Microbiology, 2008. 62: p. 193-210.

48. Avery, S.V., Microbial cell individuality and the underlying sources of heterogeneity. Nature Reviews Microbiology, 2006. 4(8): p. 577-587.

49. Dubnau, D. and R. Losick, Bistability in bacteria. Molecular Microbiology, 2006. 61(3): p. 564-572.

50. Lewis, K., Persister cells, in Annual Review of Microbiology. 2010. p. 357-372.

51. Knudsen, G.M., Y. Ng, and L. Gram, Survival of bactericidal antibiotic treatment by a persister subpopulation of Listeria monocytogenes. Applied and Environmental Microbiology, 2013. 79(23): p. 7390-7397.

52. Balaban, N.Q., J. Merrin, R. Chait, L. Kowalik, and S. Leibler, Bacterial persistence as a phenotypic switch. Science, 2004. 305(5690): p. 1622-1625.

53. van der Veen, S. and T. Abee, Bacterial SOS response: A food safety perspective. Current Opinion in Biotechnology, 2011. 22(2): p. 136-142.

54. Helaine, S. and E. Kugelberg, Bacterial persisters: formation, eradication, and experimental systems. Trends in Microbiology, 2014. 22(7): p. 417-424.

55. Abee, T., J. Koomen, K.I. Metselaar, M.H. Zwietering, and H.M.W. Den Besten, Impact of pathogen population heterogeneity and stress resistant variants on food safety. Annual Reviews in Food Science and Technology, 2016. 7:439-456.

56. Karatzas, K.A.G. and M.H.J . Bennik, Characterization of a Listeria monocytogenes Scott A isolate with high tolerance towards high hydrostatic pressure. Applied and Environmental Microbiology, 2002. 68(7): p. 3183-3189.

57. Karatzas, K.A.G., J .A. Wouters, C.G.M. Gahan, C. Hill, T. Abee, and M.H.J. Bennik, TheCtsR regulator of Listeria monocytogenes contains a variant glycine repeat region that affects piezotolerance, stress resistance, motility and virulence. Molecular Microbiology, 2003. 49(5): p. 1227-1238.

58. Karatzas, K.A.G., V.P. Valdramidis, and M.H.J . Wells-Bennik, Contingency locus in ctsR of Listeria monocytogenes Scott A: A strategy for occurrence of abundant piezotolerant isolates within clonal populations. Applied and Environmental Microbiology, 2005. 71(12): p. 8390-8396.

59. Van Boeijen, I.K.H., A.A.E. Chavaroche, W.B. Valderrama, R. Moezelaar, M.H. Zwietering, and T. Abee, Population diversity of Listeria monocytogenes LO28: Phenotypic and genotypic characterization of variants resistant to high hydrostatic pressure. Applied and Environmental Microbiology, 2010. 76(7): p. 2225-2233.

60. Van Boeijen, I.K.H., C. Francke, R. Moezelaar, T. Abee, and M.H. Zwietering, Isolation of highly heat-resistant Listeria monocytogenes variants by use of a kinetic modeling-based sampling scheme. Applied and Environmental Microbiology, 2011. 77(8): p. 2617-2624. 
61. Van Boeijen, I.K.H., P.G. Casey, C. Hill, R. Moezelaar, M.H. Zwietering, C.G.M. Gahan, and T. Abee, Virulence aspects of Listeria monocytogenes LO28 high pressure-resistant variants. Microbial Pathogenesis, 2013. 59-60: p. 48-51.

62. Tompkin, R.B., Control of Listeria monocytogenes in the food-processing environment. J ournal of Food Protection, 2002. 65(4): p. 709-725.

63. Hill, C., Virulence or niche factors: What's in a name? J ournal of Bacteriology, 2012. 194(21): p. 57255727.

64. Davis, M.J., P.J . Coote, and C.P. O’Byrne, Acid tolerance in Listeria monocytogenes: Theadaptive acid tolerance response (ATR) and growth-phase-dependent acid resistance. Microbiology, 1996. 142(10): p. 2975-2982.

65. O'Driscoll, B., C.G.M. Gahan, and C. Hill, Adaptive acid tolerance response in Listeria monocytogenes: Isolation of an acid-tolerant mutant which demonstrates increased virulence. Applied and Environmental Microbiology, 1996. 62(5): p. 1693-1698.

66. Cotter, P.D., K. O'Reilly, and C. Hill, Role of the glutamate decarboxylase acid resistance system in the survival of Listeria monocytogenes LO28 in low pH foods. J ournal of Food Protection, 2001. 64(9): p. 1362-1368.

67. Feehily, C. and K.A.G. Karatzas, Role of glutamate metabolism in bacterial responses towards acid and other stresses. J ournal of Applied Microbiology, 2013. 114(1): p. 11-24.

68. Karatzas, K.A.G., L. Suur, and C.P. O'Byrne, Characterization of the intracellular glutamate decarboxylase system: Analysis of its function, transcription, and role in the acid resistance of various strains of Listeria monocytogenes. Applied and Environmental Microbiology, 2012. 78(10): p. 35713579.

69. Ferreira, A., D. Sue, C.P. O'Byrne, and K.J . Boor, Role of Listeria monocytogenes $\sigma B$ in survival of lethal acidic conditions and in the acquired acid tolerance response. Applied and Environmental Microbiology, 2003. 69(5): p. 2692-2698.

70. Zwietering, M.H. and H.M.W. den Besten, Modelling: One word for many activities and uses. Food Microbiology, 2011. 28(4): p. 818-822.

71. Den Besten, H.M.W., M. Mataragas, R. Moezelaar, T. Abee, and M.H. Zwietering, Quantification of the effects of salt stress and physiological state on thermotolerance of Bacillus cereus ATCC 10987 and ATCC 14579. Applied and Environmental Microbiology, 2006. 72(9): p. 5884-5894.

72. Zwietering, M.H., Quantitative risk assessment: Is more complex always better? Simple is not stupid and complex is not always more correct. International J ournal of Food Microbiology, 2009. 134(1-2): p. 57-62.

73. Stumbo, C.R., Thermal bacteriology in food processing. 1973: Academic Press, NewYork.

74. Mafart, P., O. Couvert, S. Gaillard, and I. Leguerinel, On calculating sterility in thermal preservation methods: Application of the Weibull frequency distribution model. International J ournal of Food Microbiology, 2002. 72(1-2): p. 107-113.

75. Coroller, L., I. Leguerinel, E. Mettler, N. Savy, and P. Mafart, General model, based on two mixed weibull distributions of bacterial resistance, for describing various shapes of inactivation curves. Applied and Environmental Microbiology, 2006. 72(10): p. 6493-6502.

76. Whiting, R.C., Modeling bacterial survival in unfavorable environments. Journal of Industrial Microbiology, 1993. 12(3-5): p. 240-246.

77. Geeraerd, A.H., V. Valdramidis, and J.F. Van Impe, GInaFiT, a freeware tool to assess non-loglinear microbial survivor curves. International J ournal of Food Microbiology, 2005. 102(1): p. 95-105.

78. Geeraerd, A.H., V.P. Valdramidis, and J.F. Van Impe, Erratum to "GInaFiT, a freeware tool to assess non-log-linear microbial survivor curves" (vol 102, pg 95, 2005). International J ournal of Food Microbiology, 2006. 110(3): p. 297-297.

79. Nauta, M.J., Separation of uncertainty and variability in quantitative microbial risk assessment models. International J ournal of Food Microbiology, 2000. 57(1-2): p. 9-18.

80. Lammerding, A.M. and G.M. Paoli, Quantitative risk assessment: An emerging tool for emerging foodborne pathogens. Emerging Infectious Diseases, 1997. 3(4): p. 483-487.

81. Notermans, S. and P. Teunis, Quantitativerisk analysis and the production of microbiologically safe food: An introduction. International J ournal of Food Microbiology, 1996. 30(1-2): p. 3-7. 


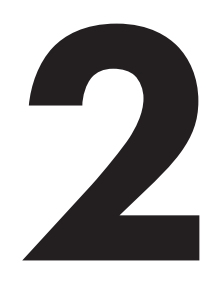

\title{
Isolation and quantification of highly acid resistant variants of Listeria monocytogenes
}

\author{
K.I. Metselaar, H.M.W. den Besten, T. Abee, \\ R. Moezelaar and M.H. Zwietering
}

Published in:

International Journal of Food Microbiology (2013) 166:508-514. 


\section{Abstract}

Heterogeneity in stress response of bacteria is one of the biggest challenges posed by minimal processing, which aims at finding the balance between microbiologically stable foods while maintaining the characteristics of fresh products. In this study, exposure of Listeria monocytogenes $\mathrm{LO} 28$ to acid stress, which can be encountered in the food processing environment as well as in the human body upon ingestion, led to inactivation kinetics showing considerable tailing, which was described by a biphasic inactivation model. Stable acid resistant variants of L. monocytogenes LO28 were isolated after exposure of late-exponential phase cells to $\mathrm{pH} 3.5$ for 90 $\mathrm{min}$. The resulting 23 stable resistant isolates could be divided in three groups: (a) highly increased acid resistance $\left(<1 \log _{10}\right.$ reduction, $\left.n=16\right)$, (b) slightly increased acid resistance (1-3 $\log _{10}$ reduction, $n=6$ ) and (c) one isolate showing a variable acid stress response. The highly acid resistant group showed increased resistance to the tested $\mathrm{pH}$ range of 2.5 to 3.5 in both late-exponential and stationary phase. Increased acid resistance showed to be significantly correlated to reduced growth rate. The Weibull model was reparameterized, resulting in improved parameter estimation, and was used to estimate the inactivation kinetics at mild $\mathrm{pH}$. Studying the growth boundaries of the wild type and a representative set of variants indicated that the increased resistance of the variants was only related to survival of severe $\mathrm{pH}$ stress but did not allow for better growth or survival at mild $\mathrm{pH}$ stress. This study shows that acid exposure of late-exponential phase cells reveals the presence of acid resistant subpopulations and that there is a phenotypic diversity amongst them. The occurrence of heterogeneity and stress resistant subpopulations may lead to a higher number of surviving microorganisms than expected. Also, stress resistant subpopulations can become part of the domestic flora in a food production line. The currently isolated acid resistant variants are a new group of stress resistant variants and underline the importance of gaining more insight in the mechanisms underlying this heterogeneity and increased resistance. 


\section{Introduction}

Listeria monocytogenes is a foodborne pathogen causing the rare but severe disease listeriosis [1]. Its robustness and ability to grow and survive under harsh conditions such as low temperature, high salt and low $\mathrm{pH}$ makes it a difficult pathogen to eliminate [2]. An additional feature that can have implications for food safety is the often observed heterogeneity in stress response of pathogens, which can result in tailing of inactivation curves [3]. Besides phenotypic heterogeneity, the observed tailing may suggest the presence of a stress resistant subpopulation [4]. Such resistant subpopulations can lead to a higher number of surviving organisms than expected after a preservation treatment, especially with the recent trend towards mild preservation. These subpopulations can also become part of the domestic flora in food processing environments, leading to product (re)contamination with these resistant organisms. Indeed, previous studies have demonstrated heterogeneity and occurrence of stress resistant variants within L. monocytogenes populations [5-7]. Exposure to heat and high hydrostatic pressure (HHP) led to considerable tailing in the inactivation curves. These inactivation kinetics could be described by a biphasic model, which assumes a stress sensitive and a stress resistant population. Isolation of survivors from the tail revealed that some were stably more resistant to the previously applied stress, while others were only temporarily more resistant. For a fraction of these stable resistant variants it was shown that they are genetically different from the main population [5]. Also, these subpopulations possessed a great phenotypic diversity. Most of the L. monocytogenes LO28 variants isolated after HHP treatment also showed increased acid resistance [8]. Acid survival is important for microorganisms both in surviving low $\mathrm{pH}$ hurdles encountered in food preservation as well as in surviving the acidic conditions encountered during gastric passage [9]. Acid resistance is therefore considered an important niche factor contributing to L. monocytogenes infection capacity [10]. A recent study by Van Boeijen et al. [11] revealed that most of the previously isolated HHP variants were less virulent than the $\mathrm{LO} 28$ wild type, but two variants which also showed increased heat and acid resistance, retained full virulence in a mouse model.

Acid inactivation of L. monocytogenes has been widely studied before [9, 12-15] and, as for heat and HHP stress, non-linearity in acid inactivation has been observed [1416]. The objectives of this study were to quantitatively describe the inactivation of late-exponential phase cells of L. monocytogenes $\mathrm{LO}_{2} 8$ upon acid exposure and to determine if a single acid exposure can lead to the selection of stable resistant variants. Although somevariants have been isolated after prolonged acid exposure of stationary phase cells [16], the occurrence and characterization of acid resistant subpopulations has notbeen studied in detail. This study highlights that L. monocytogenes populations are heterogeneous in their acid resistance resulting in biphasic inactivation kinetics and selection of highly acid resistant variants. 


\section{Materials and Methods}

\section{Bacterial strains and culturing conditions}

Listeria monocytogenes LO28 wild type (WT) (Wageningen UR Food \& Biobased Research, the Netherlands) was used in this study. This strain, originally isolated from a healthy pregnant carrier, has previously been used in our laboratory to study the presence of stress resistant subpopulations [5-7]. The stock culture was kept in $15 \%$ (v/v) glycerol (Fluka, Buchs) at $-80^{\circ} \mathrm{C}$, and before the experiments cells from stock were grown for 2 days at $30^{\circ} \mathrm{C}$ on brain heart infusion (BHI) agar (Oxoid, Hampshire). A single colony was used to inoculate $20 \mathrm{ml} \mathrm{BHI} \mathrm{broth.} \mathrm{After} \mathrm{overnight}$ (ON) growth (18-22 h) at $30^{\circ} \mathrm{C}$ (Innova 4335; New Brunswick Scientific, Edison, $\mathrm{NJ}$ ) with shaking at $160 \mathrm{rpm}, 0.5 \%(\mathrm{v} / \mathrm{v})$ inoculum was added to fresh BHI broth. Cells were grown in $\mathrm{BHI}$ at $30^{\circ} \mathrm{C}$ until late-exponential growth phase $\left(\mathrm{OD}_{600}=0.4\right.$ 0.5 (Spectrophotometer Novaspec II, Pharmacia Biotech) reached after 4-5 h) or stationary phase (18-22 $\mathrm{h}$ of growth, $\left.\mathrm{OD}_{600} \sim 2.0\right)$. Late-exponential and stationary phase cultures were used for further experiments.

\section{Inactivation kinetics at low pH}

Acid inactivation experiments were performed as previously described by Van der Veen and Abee [15] with modifications. Briefly, $100 \mathrm{ml}$ late-exponential phase culture was harvested by centrifuging for $5 \mathrm{~min}$ at $2880 \mathrm{x} \mathrm{g}$ (Centrifuge $5804 \mathrm{R}$, Eppendorf), resuspended in $10 \mathrm{ml} \mathrm{BHI}$ broth, centrifuged again for $5 \mathrm{~min}$ at 2880 $\mathrm{x} \mathrm{g}$, and resuspended in $10 \mathrm{ml} \mathrm{BHI} \mathrm{broth} \mathrm{(pre-warmed} \mathrm{at} 37^{\circ} \mathrm{C}$ ) adjusted to $\mathrm{pH} 2.5$, 3.0 or 3.5 with $10 \mathrm{M} \mathrm{HCl}$. The tube was placed in a shaking waterbath at $37^{\circ} \mathrm{C}$ and at different time intervals, samples were taken, serially diluted in BHI broth and plated on BHI agar using a spiral plater (Eddy J et, IUL S.A.). Samples were taken up to 10,60 and $200 \mathrm{~min}$ for $\mathrm{pH} 2.5,3.0$ and 3.5 respectively. Plates were incubated at $30^{\circ} \mathrm{C}$ and counted after 4- 6 days to allow recovery of all cells. Combined data of 6-9 independent experiments were used for further data analysis.

\section{Isolation of acid resistant variants}

Colonies of LO28 WT, obtained after $\mathrm{pH} 3.5$ treatment for 90 min were streaked on fresh BHI plates and incubated at $30^{\circ} \mathrm{C}$ for 2 days. Subsequently, one colony was inoculated in $10 \mathrm{ml} \mathrm{BHI}$ broth and incubated at $30^{\circ} \mathrm{C}$. Every $24 \mathrm{~h}, 0.5 \%(\mathrm{v} / \mathrm{v})$ was used to start a fresh culture, and this was repeated for 5 days in a row. This was done to assure a genetically stable population. After 5 days the culture was frozen at $-80^{\circ} \mathrm{C}$ in $15 \%$ (v/v) glycerol until further use. In total, 100 isolates from 5 experiments on 
different days were obtained with this method. Late-exponential phase cells of all isolates were then retested for acid resistance. For this, isolates were grown to lateexponential phase and harvested by centrifuging $50 \mathrm{ml}$ of cell suspension for $5 \mathrm{~min}$ at $2880 \mathrm{x}$ g. The cells were resuspended in $5 \mathrm{ml} \mathrm{BHI} \mathrm{broth,} \mathrm{centrifuged} \mathrm{again} \mathrm{for} 5$ min at $2880 \mathrm{x} \mathrm{g}$ and resuspended in $0.5 \mathrm{ml}$ PPS $(0.1 \% \mathrm{w} / \mathrm{v}$ bacteriological peptone (Oxoid, Hampshire, England) and $0.8 \% \mathrm{w} / \mathrm{v} \mathrm{NaCl}$ (Merck)). This cell suspension was added to a tube, containing $4.5 \mathrm{ml}$ prewarmed BHI adjusted to $\mathrm{pH} 2.5$ by $10 \mathrm{M}$ $\mathrm{HCl}$ and a small magnetic stirring bar. This tube was placed in a small flask, in which water of $37^{\circ} \mathrm{C}$ was pumped, to ensure a constant temperature and accurate mixing of the cells using a magnetic stirrer. Samples were taken at $\mathrm{t}=0$ (just after adding the cells to the $\mathrm{BHI}$ ) and $3.5 \mathrm{~min}$, serially diluted in $\mathrm{BHI}$ to restore neutral $\mathrm{pH}$ and plated on $\mathrm{BHI}$ agar using a spiral plater and incubated for 4-6 days at $30^{\circ} \mathrm{C}$. Isolates that showed increased acid resistance (more than $1 \log _{10}$ higher survival compared to the WT) in late-exponential phase were tested at least three times on different days, to determine whether they were stably more acid resistant. Afterwards, the stable acid resistant variants were also tested three times for acid resistance in stationary phase. The same procedure was followed, with the modification that $10 \mathrm{ml}$ cell suspension was used and samples were taken after $15 \mathrm{~min}$ of exposure to $\mathrm{pH} 2.5$, to obtain a similar reduction as late-exponential phase cells after $3.5 \mathrm{~min}$. The mean $\log _{10}$ reduction was calculated by subtracting the $\log _{10}$ count after acid exposure from the initial $\log _{10}$ count. Acid resistance of the tested isolates was compared to the acid resistance of the WT by performing a Student t-test on the mean reduction in $\log _{10}$ count after 3.5 or $15 \mathrm{~min}$ at $\mathrm{pH} 2.5$ for late-exponential and stationary phase cells respectively.

\section{Model fitting}

A biphasic model with shoulder [17-19] was fitted to the inactivation data using TableCurve 2D (version 5.01 for Windows) as is shown in equation 1.

$$
\begin{aligned}
& \log _{10}\left(N_{t}\right)=\log _{10}\left(N_{0}\right)+\log _{10}\left((1-f) \cdot \exp \left(-k_{\text {sens }} \cdot t\right) \cdot \frac{\exp \left(k_{\text {sens }} \cdot S_{l}\right)}{1+\left(\exp \left(k_{\text {sens }} \cdot S_{l}\right)-1\right) \cdot \exp \left(-k_{\text {sens }} \cdot t\right)}\right. \\
& \left.+f \cdot \exp \left(-k_{\text {res }} \cdot t\right) \cdot\left(\frac{\exp \left(k_{\text {sens }} \cdot S_{l}\right)}{1+\left(\exp \left(k_{\text {sens }} \cdot S_{l}\right)-1\right) \cdot \exp \left(-k_{\text {sens }} \cdot t\right)}\right)^{\frac{k_{\text {res }}}{k_{\text {sens }}}}\right)
\end{aligned}
$$

In which $\mathrm{N}_{\mathrm{t}}$ is the number of cells at time $\mathrm{t}$ (in min), $\mathrm{N}_{0}$ is the number of cells at $t=0$, $\mathrm{k}_{\text {sens }}$ and $\mathrm{k}_{\mathrm{res}}$ are the specific inactivation rates of the sensitive population and the 
resistant fraction, respectively $\left(\mathrm{min}^{-1}\right)$. $\mathrm{S}_{1}$ is the shoulder length (in $\mathrm{min}$ ) and $\mathrm{f}$ is the stress resistant fraction. For all further calculations, the mean parameter estimates of the TableCurve output were used. The D-lue of both subpopulations was calculated from their inactivation rate, without taking the shoulder into account:

$$
D=\frac{\ln (10)}{k}
$$

An F test was used to test if the fitting performance of the model was statistically accepted. The f value was calculated using the following equation [19]:

$$
f=\frac{\text { MSE }_{\text {model }}}{\mathrm{MSE}_{\text {data }}}
$$

In which $\mathrm{MSE}_{\text {model }}$ is the mean square error of the model and $\mathrm{MSE}_{\text {data }}$ the mean square error of the data for replicate values. The resulting f value was tested against a critical F table value (95\% confidence). A lower f value than the critical Fvalue indicated that the model describes the data well.

\section{Reduction of model parameters}

A stepwise procedure was followed to reduce the number of parameters of the model (equation 1), as described by Den Besten et al. [19]. Briefly, a t-test was used to test the significance of the parameter estimates. Parameters that were not significant were stepwise removed from the model until all parameters were significant. Additionally, a lack-of-fit test was performed to confirm if the reduction of the parameters was statistically acceptable. The f value to test if a parameter could be excluded was calculated by the following equation:

$$
f=\frac{\left(R S S_{2}-R S S_{1}\right) /\left(D F_{2}-D F_{1}\right)}{R S S_{1} / D F_{1}}
$$

In which $\mathrm{RSS}_{1}$ and $\mathrm{RSS}_{2}$ are the residual sum of squares of the full model and the reduced model respectively and $\mathrm{DF}_{1}$ and $\mathrm{DF}_{2}$ the degrees of freedom of both models.

\section{Maximum specific growth rate}

$50 \mu \mathrm{l} \mathrm{ON}$ culture of WT and all 23 variants was inoculated in $10 \mathrm{ml} \mathrm{BHI}$ or BHI with $\mathrm{pH}$ adjusted to 5.0 by $10 \mathrm{M} \mathrm{HCl}$, at an initial concentration of $\sim 10^{7} \mathrm{cfu} / \mathrm{ml}$. $200 \mathrm{pl}$ of this culture was transferred to a 96-wells plate in triplicate for each variant and the WT. BHI and BHI pH 5.0 were used as blanks. Growth was monitored by incubating the 96-wells plate in a spectrophotometer (Tecan Infinite F200, Tecan Group Ltd.) set at $30^{\circ} \mathrm{C}$ and measuring the $\mathrm{OD}_{595}$ every $10 \mathrm{~min}$ for $24 \mathrm{~h}$, with $5 \mathrm{sec}$ of shaking before each measurement. The blanks were subtracted from the readings 
and the natural logarithm of the OD values was calculated. The maximum specific growth rate $\left(\mu_{\max }\right)$ was calculated by taking the slope of the $\ln (\mathrm{OD})$ against time $(\mathrm{h})$. Experiments were performed in biological duplicates.

\section{Minimum pH for growth}

In order to determine the minimum $\mathrm{pH}$ at which the WT and 7 selected variants were able to grow, ON cultures were inoculated in erlenmeyer flasks containing BHI with the $\mathrm{pH}$ adjusted to $4.2,4.3,4.4$ and 4.5 by $10 \mathrm{M} \mathrm{HCl}$, at an initial concentration of $\sim 10^{7}$ cells $/ \mathrm{ml}$. Flasks were incubated at $30^{\circ} \mathrm{C}$ with aeration at $160 \mathrm{rpm}$. Growth or inactivation was monitored up to 8 days by $24-72 \mathrm{~h}$ interval sampling. Samples were serially diluted in $\mathrm{BHI}$ and plated on $\mathrm{BHI}$ agar. Plates were incubated at $30^{\circ} \mathrm{C}$ and counted after 4- 6 days of incubation. Experiments were performed in duplicate. Inactivation data were compared by fitting a Weibull model [20] (equation 5) and a reparameterized Weibull model (equation 6) to the replicate data:

$$
\log _{10}\left(N_{t}\right)=\log _{10}\left(N_{0}\right)-\left(\frac{t}{\delta}\right)^{\beta}
$$

in which $\delta$ the time needed for the first decimal reduction (h); and $\beta$, a fitting parameter that defines the shape of the curve.

$$
\log _{10}\left(N_{t}\right)=\log _{10}\left(N_{0}\right)-\Delta \cdot\left(\frac{t}{t_{\Delta D}}\right)^{\beta}
$$

in which $\Delta$ is the number of decimal reductions; $\mathrm{t}_{\Delta \mathrm{D}^{\mathrm{D}}}$, the time needed to reduce the initial number of microorganism with $\Delta$ decimals (h); and $\beta$, a fitting parameter that defines the shape of the curve. $\Delta$ was set at 3 and the other parameters were estimated by TableCurve 2D version 5.01 for Windows. The correlation between the model parameters was investigated using Matlab (version R2012a). The estimates for parameter $t_{3 D^{\prime}}$, the time needed to reach a $3 \log _{10}$ reduction, were compared by a Student t-test.

\section{Results and discussion}

\section{Acid inactivation kinetics}

In this study L. monocytogenes $\mathrm{LO} 28$ was inactivated at $\mathrm{pH} 2.5,3.0$ and 3.5 and at all tested $\mathrm{pH}$ conditions the inactivation curves showed considerable tailing (Figure 2.1). The shape of the inactivation data suggested the presence of an acid sensitive 
and an acid resistant population. Therefore, a biphasic model (equation 1) was fitted to the data and an F-test confirmed that the fit was acceptable for all tested $\mathrm{pH}$ conditions ( $\mathrm{p}$-value of $0.146,0.401$ and 0.442 for $\mathrm{pH} 2.5,3.0$ and 3.5 respectively).
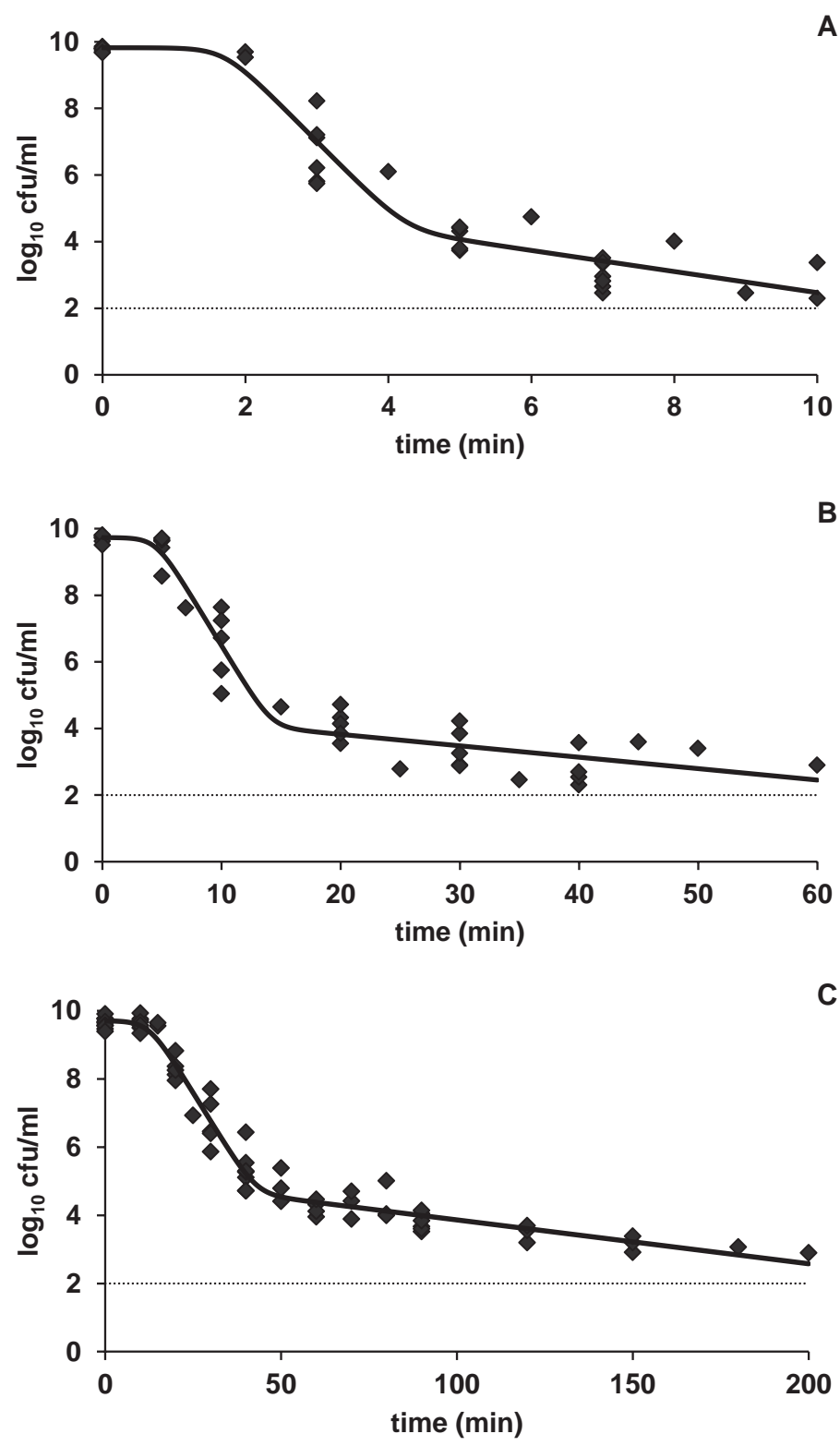

Figure 2.1: Inactivation kinetics of late-exponential phase cells of L. monocytogenes LO28 WT in BHI adjusted with $\mathrm{HCl}$ to $\mathrm{pH} 2.5$ (A), pH 3.0 (B) and $\mathrm{pH} 3.5(\mathrm{C})$ at $37^{\circ} \mathrm{C}$. A biphasic model with shoulder is fitted to the inactivation data [17-19]. The horizontal line represents the detection limit. 
However, the parameter estimation for $\mathrm{f}$ was not found significant for $\mathrm{pH} 2.5$ and 3.0, and only slightly significant for $\mathrm{pH} 3.5(\mathrm{p}=0.039)$ despite the clearly observed tailing (see Figure 2.1). Removing this parameter, and thereby also removing the parameter $\mathrm{k}_{\text {res }}$ did not result in a better fit according to the F-test. It has been reported before that this biphasic model cannot significantly estimate the resistant fraction while clear tailing was observed, but removing this parameter was not acceptable either [19]. To date, this model has been widely used to describe biphasic inactivation kinetics, but in many cases without reporting the values or significance of the parameter estimate for $f$ [21-23]. Since the initial number of cells $\left(\mathrm{N}_{0}\right)$ was estimated on a $\log _{10}$ scale, we considered it plausible to also estimate $\mathrm{f}$ on a $\log _{10}$ scale. This resulted in the following equation:

$$
\begin{aligned}
& \log _{10}\left(N_{t}\right)=\log _{10}\left(N_{0}\right)+\log 10\left(\left(1-10^{\log _{10}(f)}\right) \cdot \exp \left(-k_{\text {sens }} \cdot t\right) \cdot \frac{\exp \left(k_{\text {sens }} \cdot S_{l}\right)}{1+\left(\exp \left(k_{\text {sens }} \cdot S_{l}\right)-1\right) \cdot \exp \left(-k_{\text {sens }} \cdot t\right)}\right. \\
& \left.+10^{\log _{\text {I10 }}(f)} \cdot \exp \left(-k_{\text {res }} \cdot t\right) \cdot\left(\frac{\exp \left(k_{\text {sens }} \cdot S_{l}\right)}{1+\left(\exp \left(k_{\text {sens }} \cdot S_{l}\right)-1\right) \cdot \exp \left(-k_{\text {sens }} \cdot t\right)}\right)^{\frac{k_{\text {ress }}}{k_{\text {sens }}}}\right)
\end{aligned}
$$

Fitting this adjusted model to the inactivation data resulted in the same parameter estimates, but with a significant estimate for $\log _{10}(\mathrm{f})$. We therefore propose to estimate $\log _{10}(\mathrm{f})$ instead of $\mathrm{f}$ when using this biphasic model. The parameter estimates obtained by equation 7 are shown in Table 2.1. The estimated resistant fractions were comparable at the tested $\mathrm{pH}$ conditions and this resistant fraction was also comparable to the resistant fraction that was found for the same strain exposed to HHP stress [6]. The D-values of the sensitive population at $\mathrm{pH} 2.5$, 3.0 and 3.5 were $0.46,1.70$ and 5.99 min, respectively. The resistant fraction was characterized by a higher decimal reduction time of around 10-fold (7-17 fold) with D-values of 3.16, 29.2 and $77.8 \mathrm{~min}$ respectively. It can be concluded that, after $\log _{10}$-transformation of $\mathrm{f}$, the biphasic model with shoulder is a suitable model to describe acid inactivation of L. monocytogenes $\mathrm{LO}_{2} 8$ and estimate the fraction of resistant cells. The significant tailing suggested that stress resistant cells could be isolated from the tail. 
Table 2.1: Fitted model parameters for adjusted biphasic inactivation model (equation 7 ) with shoulder for L. monocytogenes LO28 WT in BHI adjusted to $\mathrm{pH} 2.5,3.0$ and 3.5 at $37^{\circ} \mathrm{C}$. The $95 \%$ confidence interval of each parameter estimate is indicated between brackets

\begin{tabular}{|llll|}
\hline parameter & $\mathbf{p H} 2.5$ & $\mathbf{p H} 3.0$ & $\mathbf{p H ~ 3 . 5}$ \\
\hline $\log _{10} N_{0}$ & 9.8 & 9.7 & 9.7 \\
& {$[9.4 ; 10.3]$} & {$[9.2 ; 10.3]$} & {$[9.5 ; 9.9]$} \\
$k_{\text {sens }}\left(\mathrm{min}^{-1}\right)$ & 4.98 & 1.35 & 0.38 \\
& {$[2.66 ; 7.30]$} & {$[0.89 ; 1.82]$} & {$[0.33 ; 0.44]$} \\
$k_{\text {res }}\left(\mathrm{min}^{-1}\right)$ & 0.73 & 0.08 & 0.03 \\
& {$[0.25 ; 1.21]$} & {$[0.02 ; 0.14]$} & {$[0.02 ; 0.04]$} \\
Shoulder $(\mathrm{min})$ & 1.7 & 4.5 & 12.4 \\
& {$[1.04 ; 2.36]$} & {$[2.48 ; 6.46]$} & {$[9.15 ; 15.57]$} \\
Resistant fraction & $1.89 \cdot 10^{-5}$ & $4.19 \cdot 10^{-6}$ & $1.93 \cdot 10^{-5}$ \\
& {$\left[1.20 \cdot 10^{-6} ; 3.02 \cdot 10^{-4}\right]$} & {$\left[5.01 \cdot 10^{-7} ; 3.47 \cdot 10^{-5}\right]$} & {$\left[7.41 \cdot 10^{-6} ; 5.01 \cdot 10^{-5}\right]$} \\
\hline
\end{tabular}

\section{Stable acid resistant variants}

To explore whether acid treatment resulted indeed in selection of stableacid resistant variants, 100 colonies obtained after acid treatment of 90 min at pH 3.5 were subcultured five times and therefore considered to have a stable genotype. Retesting in late-exponential phase resulted in 23 out of 100 isolates with a significantly lower reduction in viable counts after severe acid treatment ( $\mathrm{pH} 2.5$ for $3.5 \mathrm{~min}$ ) than the $\mathrm{WT}$ and were therefore considered to have a stable acid resistant phenotype (Figure $2.2 \mathrm{~A}$ ). Sixteen of the 23 variants were highly resistant for a treatment of $\mathrm{pH} 2.5$ for $3.5 \mathrm{~min}$, showing less than $1 \log _{10}$ reduction after acid treatment, compared to an average of $4 \log _{10}$ reduction for the WT. Six isolates were slightly but significantly more acid resistant than the WT, with a reduction of $2-3 \log _{10}$ after 3.5 min at pH 2.5. One variant, number 7 , was tested several times, showed increased acid resistance in all cases, but the variability between experiments was relatively large with a reduction ranging from 0.6 to $2.7 \log _{10} \mathrm{cfu} / \mathrm{ml}$. Subsequently, all 23 stable resistant variants were tested for acid resistance in stationary phase as well. 

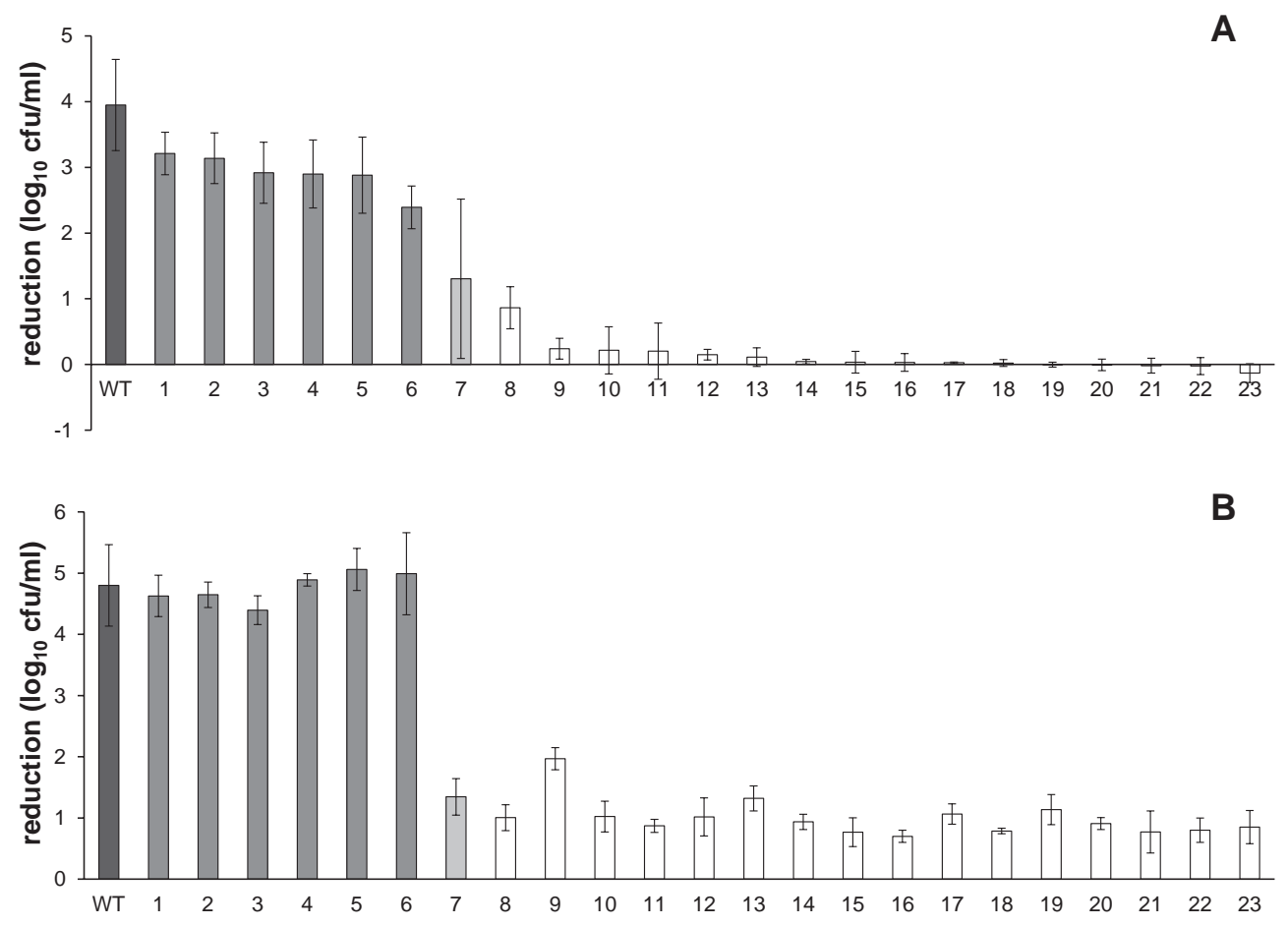

Figure 2.2: Acid resistance of L. monocytogenes $\mathrm{LO}_{2} 8 \mathrm{WT}$ and stable resistant variants. Acid resistance is expressed as the reduction in $\log _{10} \mathrm{cfu} / \mathrm{ml}$ after $\mathrm{pH} 2.5$ treatment for $3.5 \mathrm{~min}$ for late-exponential (A) and $15 \mathrm{~min}$ for stationary phase (B) cells of L. monocytogenes LO28 WT and variants obtained after 90 $\mathrm{min}$ at $\mathrm{pH}$ 3.5. Error bars represent the standard deviation of the mean reduction of at least 3 independent experiments.

It is generally known that acid resistance is growth phase dependent [14] and therefore it was expected that cells grown until stationary phase would be more resistant to acid stress. Both the WT and the 23 variants were indeed more resistant to $\mathrm{pH} 2.5$ in stationary phase and longer inactivation time was necessary to obtain a relevant reduction. Figure 2.2B shows a similar pattern in acid resistance for stationary phase cells as for late-exponential phase cells. The highly resistant group could clearly be distinguished, showing a $1-2 \log _{10}$ reduction after $15 \mathrm{~min}$ at $\mathrm{pH} 2.5$, while the WT showed around $5 \log _{10}$ reductions. Variant 9 of the highly resistant group, is significantly less resistant than the other variants within this group in stationary phase. The slightly resistant group did not show a significantly increased resistance to $\mathrm{pH} 2.5$ in stationary phase. Variant 7, that showed a quite high variation in acid resistance during late-exponential phase, which is most likely growth phase related [14, 24], falls clearly within the group of highly acid resistant variants in stationary phase, confirming the stable acid resistance of this isolate. Inactivation kinetics in time were studied in more detail for 2 variants. Besides the increased 
acid shock survival at pH 2.5, variants 7 and 15 showed increased resistance over a prolonged period of time (Figure 2.3) at $\mathrm{pH} 2.5,3.0$ and 3.5. In order to quantify the difference in inactivation kinetics, equation 7 as well as the reduced models were fitted to the data, until all parameters were significant. This approach resulted in fitting the linear inactivation model to variant 15 for all $\mathrm{pH}$ conditions. This was in line with previous results, since linear inactivation was also observed for resistant L. monocytogenes variants isolated after HHP treatment which were exposed to HHP again [5]. The parameter estimates when fitting the full model for variant 7 at $\mathrm{pH}$ 2.5 were marginally significant ( $\mathrm{p}$-value of 0.03 and 0.04 for $\mathrm{k}_{\text {res }}$ and $\mathrm{f}$ respectively) and therefore a lack-of-fit test was performed to determine if the parameters could be reduced. The lack-of-fit test accepted the elimination of the shoulder and the tail, and therefore the linear inactivation model was selected to fit the data at $\mathrm{pH} 2.5$. At $\mathrm{pH} 3.0$ the parameters could not be excluded and the full model was applied. At pH 3.5, both thet-test and the lack-of-fit test indicated the linear model to be acceptable.

The parameter estimates (Table 2.2) indicated that the variants 7 and 15 were indeed more acid resistant than the WT at pH 2.5, 3.0 and 3.5 over a prolonged period of time. The $\mathrm{k}$ of variant 15 at $\mathrm{pH} 2.5$ and 3.5 was rather similar to $\mathrm{k}_{\mathrm{res}}$ of the WT at $\mathrm{pH}$ 2.5 and 3.5. Interestingly, the $\mathrm{k}$ of variant 7 is higher than $\mathrm{k}_{\mathrm{res}}$ of the WT at $\mathrm{pH} 2.5$, but when the $\mathrm{pH}$ is increasing, the $\mathrm{k}_{\mathrm{res}}(\mathrm{pH} 3.0)$ and $\mathrm{k}$ (pH 3.5) of variant 7 are similar to the $\mathrm{k}_{\mathrm{res}}$ of the WT and the $\mathrm{k}$ of variant 15. The fact that $\mathrm{k}_{\mathrm{res}}$ of the WT population is not exactly the same as the $\mathrm{k}$ of the variants can be explained by the assumption that there are multiple subpopulations present in the tail, as is also clear from Figure 2.2.

Table 2.2: Fitted model parameters for adjusted biphasic inactivation model with shoulder and linear inactivation model $[17,18]$ for L. monocytogenes variants 7 and 15 in BHI adjusted to $\mathrm{pH} 2.5,3.0$ and 3.5 at $37^{\circ} \mathrm{C}$. The $95 \%$ confidence interval of each parameter estimate is indicated between brackets

\begin{tabular}{|c|c|c|c|c|}
\hline & parameter & pH 2.5 & pH 3.0 & pH 3.5 \\
\hline \multirow[t]{5}{*}{ Variant 7} & $\log _{10} N_{0}$ & $\begin{array}{l}9.76 \\
{[8.91,10.62]}\end{array}$ & $\begin{array}{l}9.68 \\
{[9.32,10.05]}\end{array}$ & $\begin{array}{l}9.74 \\
{[9.61,9.88]}\end{array}$ \\
\hline & $k_{\text {sens }}\left(\min ^{-1}\right)$ & - & $\begin{array}{l}0.36 \\
{[0.24,0.49]}\end{array}$ & - \\
\hline & $k_{\text {res }}\left(\min ^{-1}\right)^{\mathrm{a}}$ & $\begin{array}{l}1.48 \\
{[1.17,1.80]}\end{array}$ & $\begin{array}{l}0.10 \\
{[0.02,0.18]}\end{array}$ & $\begin{array}{l}0.02 \\
{[0.01,0.02]}\end{array}$ \\
\hline & Shoulder (min) & - & $\begin{array}{l}7.1 \\
{[1.30,12.96]}\end{array}$ & - \\
\hline & Resistant fraction & & $\begin{array}{l}1.31 \cdot 10^{-3} \\
{\left[3.39 \cdot 10^{-5}, 5.0\right.}\end{array}$ & \\
\hline \multirow[t]{2}{*}{ Variant 15} & $\log _{10} N_{0}$ & $\begin{array}{l}10.08 \\
{[9.45,10.71]}\end{array}$ & $\begin{array}{l}10.00 \\
{[9.65,10.35]}\end{array}$ & $\begin{array}{l}9.64 \\
{[9.49,9.79]}\end{array}$ \\
\hline & $k\left(\min ^{-1}\right)$ & $\begin{array}{l}0.77 \\
{[0.62,0.92]}\end{array}$ & $\begin{array}{l}0.16 \\
{[0.13,0.19]}\end{array}$ & $\begin{array}{l}0.01 \\
{[0.01,0.02]}\end{array}$ \\
\hline
\end{tabular}

${ }^{\text {a }}$ Parameter $\mathrm{k}\left(\mathrm{min}^{-1}\right)$ for variant 7 at $\mathrm{pH} 2.5$ and 3.5 

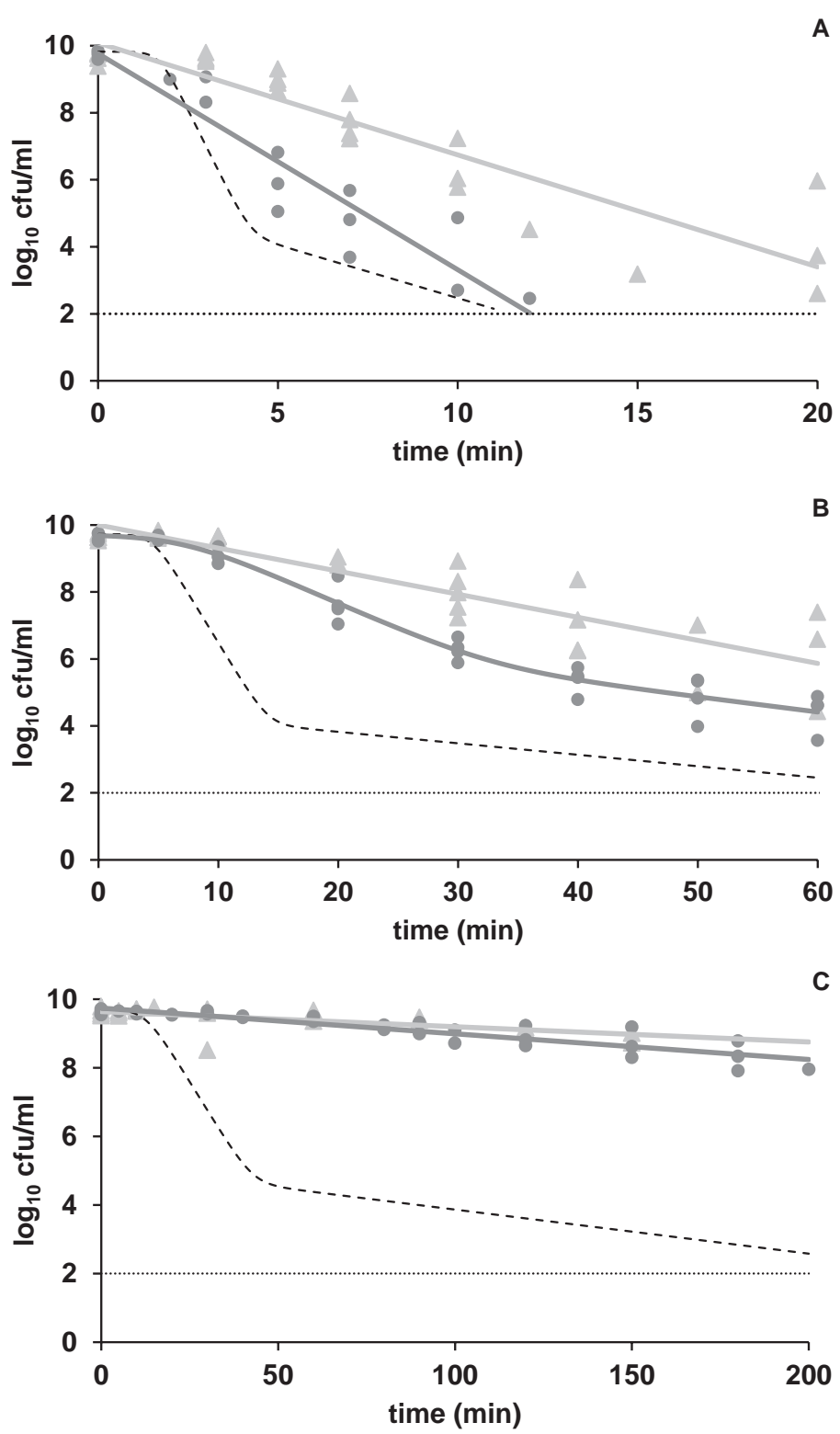

Figure 2.3: Inactivation kinetics of late-exponential phase cells of L. monocytogenes variant $7(\bullet)$ and variant $15(\triangle)$ in $\mathrm{BHI}$ adjusted with $\mathrm{HCl}$ to $\mathrm{pH} 2.5(\mathrm{~A}), \mathrm{pH} 3.0$ (B) and $\mathrm{pH} 3.5(\mathrm{C})$ at $37^{\circ} \mathrm{C}$. The biphasic model with shoulder, or a reduced model is fitted to the data and represented by a light gray (variant 15) or dark gray (variant 7) line. The fit of the biphasic model to the WT, obtained from figure 2.1, is indicated by the dotted line. The horizontal line represents the detection limit.

The $_{\text {res }}$ of the WT is therefore a combination of all the different isolates obtained from the tail. 23 of the 100 isolates were stably more resistant while the other isolates were only temporarily more resistant, most likely as a result of phenotype switching [25]. 
The resistant fraction within the WT population exposed to $\mathrm{pH} 3.5$ was estimated to be between $5.0 \cdot 10^{-5}$ and $7.4 \cdot 10^{-6}$ (Table 2.1) and therefore the stable resistant fraction ( $23 \%$ of the tested isolates) can be estimated to be between $1.2 \cdot 10^{-5}$ and $1.7 \cdot 10^{-6}$. This value is similar to the stable resistant fraction of variants obtained with HHP treatment of L. monocytogenes LO28 found by Van Boeijen et al. [6].

\section{Growth under optimal and mild pH conditions}

To determine any difference in growth behavior under optimal conditions, as well as under mild $\mathrm{pH}$ stress, growth of the $\mathrm{WT}$ and 23 stable resistant isolates was monitored in $\mathrm{BHI}$ and in $\mathrm{BHI}$ at $\mathrm{pH}$ 5.0. The highly resistant group of variants showed a slightly reduced growth rate in BHI (see Figure 2.4). The only exceptional variant with regard to growth is number 9 . This variant has a strongly reduced growth rate, which is about half the growth rate of theWT in BHI as well as in BHI set at pH 5.0 at $30^{\circ} \mathrm{C}$. Also, this variant was less acid resistant in stationary phase than the rest of the highly resistant group. Therefore this variant was excluded from linear regression at both optimal $\mathrm{pH}$ and mild $\mathrm{pH}$ stress.
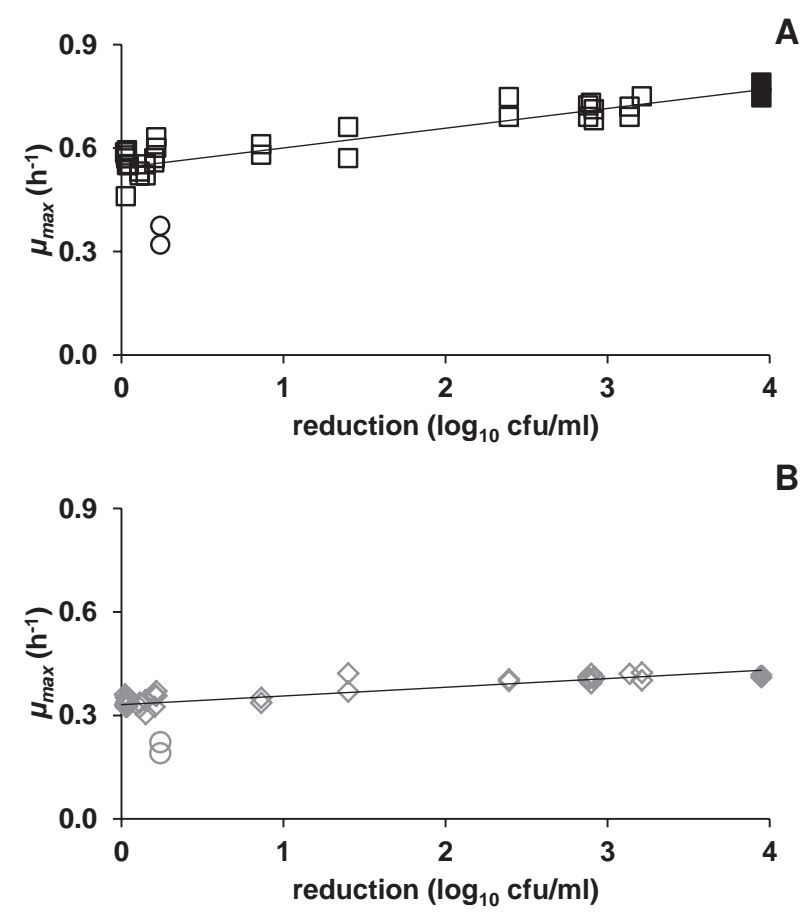

Figure 2.4: Maximum specific growth rates $\left(\mathrm{h}^{-1}\right)$ of WT (closed symbols) and 23 stable resistant variants (open symbols) in $\mathrm{BHI}(\mathrm{A})$ and in $\mathrm{BHI}$ set at $\mathrm{pH} 5.0$ by $10 \mathrm{M} \mathrm{HCl}$ (B) at $30^{\circ} \mathrm{C}$ plotted against the reduction in viable count of late-exponential phase cells at $\mathrm{pH} 2.5$ for $3.5 \mathrm{~min}$. Variant 9 is excluded from linear regression due to its particular behaviour (see text for explanation) and is indicated by 0 . 
A slight but significant correlation was found between acid resistance and maximum specific growth rate with a p-value of 0.000 and $R^{2}$ is 0.80 (if variant 9 is included in the regression the regression coefficients remain almost equal, only $\mathrm{R}^{2}$ reduces to 0.63 ). At $\mathrm{pH}$ 5.0 there is a slight but significant correlation between growth rate and acid resistance ( $p$-value of 0.000 and $R^{2}$ is 0.81 , if variant 9 is included in the regression $\mathrm{R}^{2}$ reduces to 0.53), but the regression coefficient (0.023) is lower than at neutral $\mathrm{pH}$ (0.053), suggesting that the effect observed at neutral $\mathrm{pH}$ is not so distinct at lower $\mathrm{pH}$. The observed reduction in growth rates of the highly acid resistant variants at optimal conditions is in line with reduced growth rates for some of the HHP resistant variants described in earlier studies [7, 8]. However, previously isolated acid resistant mutants from stationary phase [16] showed no difference in growth rate compared to the WT under optimal conditions but an increased growth rate at mild $\mathrm{pH}$ stress. In contrast to these previously isolated mutants, our variants showed similar or even slightly reduced growth rates at $\mathrm{pH} 5.0$, suggesting that a different type of variants was isolated.

\section{Behaviour around the growth boundary}

In order to determine if the increased resistance to severe $\mathrm{pH}$ might also be related to increased survival around the growth/no growth boundary, a representative set of 7 variants was selected based on their acid resistance, growth characteristics and experiment of isolation. All variants and the WT were able to grow at $\mathrm{pH} 4.5$ (data not shown). The growth/no growth boundary of the WT and all variants was determined to be at $\mathrm{pH} 4.4$. At $\mathrm{pH} 4.3$ and $4.2 \mathrm{a}>3 \log _{10}$ reduction was observed for all variants and the WT within 6-8 days.

Table 2.3: Correlation coefficients between the parameters $\beta$ and $\delta$ (equation 5), and $\beta$ and $t_{3 D}$ (equation 6) for the WT and 7 variants in $\mathrm{BHI}$ at $30^{\circ} \mathrm{C}$ set at $\mathrm{pH} 4.2$ or 4.3 by $10 \mathrm{M} \mathrm{HCl}$

\begin{tabular}{|lcccc|}
\hline & $\mathrm{pH} 4.2$ & $\mathrm{pH} 4.3$ & \\
\cline { 2 - 5 } & $\beta$ and $\delta$ & $\beta$ and $t_{3 D}$ & $\beta$ and $\delta$ & $\beta$ and $t_{3 D}$ \\
\hline WT & 0.95 & 0.38 & 0.95 & 0.01 \\
Variant 3 & 0.86 & -0.64 & 0.97 & 0.42 \\
Variant 7 & 0.60 & -0.91 & 0.95 & -0.25 \\
Variant 9 & 0.89 & -0.52 & 0.96 & 0.40 \\
Variant 13 & 0.94 & -0.25 & 0.96 & 0.29 \\
Variant 14 & 0.95 & -0.08 & 0.94 & -0.42 \\
Variant 15 & 0.94 & -0.35 & 0.95 & -0.21 \\
Variant 23 & 0.94 & -0.18 & 0.95 & -0.07 \\
\hline
\end{tabular}


The inactivation data showed a downward concave shape [20] and therefore the Weibull model (equation 5) was fitted to the data. In this model however, the parameters $\beta$ and $\delta$ are known to be structurally strongly correlated [20]. This was also the case for the variants at $\mathrm{pH} 4.3$ and for most of the variants at $\mathrm{pH}$ 4.2 (Table 2.3). Therefore, a reparameterized Weibull model (equation 6) was fitted to the inactivation data. In this adjusted version of the model, the time to the first $\Delta$ decimal reductions is calculated instead of the time to the first decimal reduction. By setting $\Delta$ at 3 , the parameter correlations were acceptable $(<0.95)$ for all cases and allowed for parameter estimation without extrapolating out of the experimental range. In food industry, the time to 3 decimal reductions is more relevant than the time to the first decimal reduction. Therefore the parameter $t_{3 \mathrm{D}}$ was used to quantitatively compare the behaviour of different strains. Parameter $\beta$ was comparable between the WT and the variants, ranging from 1.76 to 2.73 at $\mathrm{pH}$ 4.2 and ranging from 1.73 to 2.54 at $\mathrm{pH} 4.3$. The estimations for parameter $\mathrm{t}_{3 \mathrm{D}}$ are shown in Figure 2.5. Unlike at severe $\mathrm{pH}$, there was no clear distinction between the WT and the different groups of variants at $\mathrm{pH}$ values close to the growth boundary. Although some variants did show a slightly significantly different $t_{3 D}$ value, the inactivation of the WT and variants at mild $\mathrm{pH}$ stress ( $\mathrm{pH} 4.2$ and 4.3) was much more similar than at more severe $\mathrm{pH}$ stress (Figure 2.5). This leads to the conclusion that the mechanisms responsible for the increased acid resistance are most likely only related to the survival during severe $\mathrm{pH}$ stress.

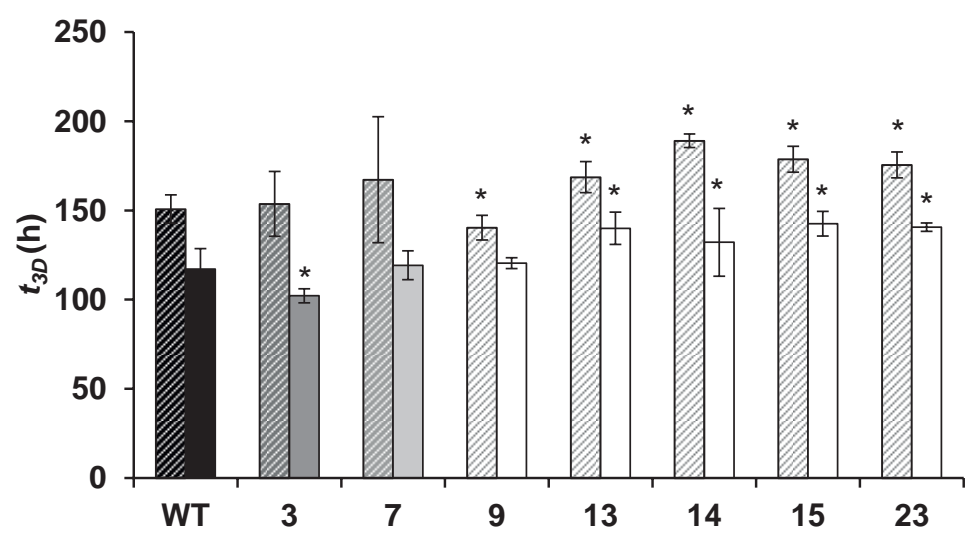

Figure 2.5: Time to the first $3 \log _{10}$ reductions $\left(t_{3 \mathrm{D}}\right)$ for $\mathrm{WT}$ and a set of resistant variants in BHI set at $\mathrm{pH} 4.2$ (solid bars) and $\mathrm{pH} 4.3$ (dashed bars) at $30^{\circ} \mathrm{C}$. The $\mathrm{t}_{3 \mathrm{D}}$-value is the parameter estimate obtained by fitting a reparameterized Weibull model through the data of a duplicate experiment. Error bars represent the $95 \%$ confidence interval of the parameter estimate. Significant differences from the WT $(p<0.05)$ are indicated by*. 


\section{Implications of acid resistant subpopulations}

Acid is an important hurdle in both food preservation and gastric passage of pathogens [9]. Several researchers reported the occurrence of stress resistant subpopulations upon inactivation treatment using different types of stress [5, 6, 16]. Here, we show that also acid treatment of late-exponential phase cells results in the selection of highly resistant variants of L. monocytogenes LO28. We have described and compared the acid inactivation kinetics of the WT and the obtained acid resistant variants. It is known that stress resistance of $\mathrm{L}$. monocytogenes is subject to strain variability [26]. In a study from Dykes and Moorhead [27] 15 clinical isolates were tested on their acid survival (stationary phase, $2 \mathrm{~h}$ at $\mathrm{pH}$ 2.5). All isolates showed less than $1 \log _{10}$ reduction over this time period, making them more resistant than LO28 WT and the currently isolated variants. It is not surprising that clinical isolates, which apparently survived the stomach, displayed such high acid resistance. The acid resistance of the currently isolated variants is comparable to the acid resistance of LO28 HHP variants [8]. Although LO28 is not amongst the most acid resistant L. monocytogenes strains, this study shows that variants with highly increased acid resistance can be selected for by acid stress exposure. The increased survival in both late-exponential and stationary phase can be highly beneficial for the organism to survive gastric passage. This relevance is underlined by the generally high acid resistance of clinical isolates. Mild acid stress adaptation might increase resistance towards lethal acid stress even further [14, 28]. Although the variants did not show better growth at food relevant $\mathrm{pH}$, they do have a better chance of survival at lowpH. The low inactivation constant at $\mathrm{pH} 2.5$ by which variant 15 and probably also the other highly resistant variants are characterized, may increase the chances that even low numbers of microorganisms present in food products will pass the stomach and can subsequently cause infection in humans.

This study showed that there is a phenotypic diversity amongst the selected acid resistant subpopulations. The differences in acid resistance and growth behaviour observed between previously isolated variants and the currently isolated variants suggest that different types of stress resistant variants are selected for under different conditions. Interestingly, the resistant fraction found in this study is very similar to the resistant fraction found after HHP treatment [6]. Quantification of the inactivation data and the resistant fraction allows for prediction of the stable resistant fraction within a population. The mechanisms underlying the increased acid resistance remain to be elucidated. This group of variants extends the group of previously isolated variants from different types of stress under different conditions and underlines the importance of gaining more insights in their characteristics and how these stress resistant subpopulations are being generated within a population. 


\section{References}

1. Cossart, P., Listeriology (1926-2007): the rise of a model pathogen. Microbes and Infection, 2007. 9(10): p. 1143-1146.

2. Hill, C., P.D. Cotter, R.D. Sleator, and C.G.M. Gahan, Bacterial stress response in Listeria monocytogenes: J umping the hurdles imposed by minimal processing. International Dairy J ournal, 2002. 12(2-3): p. 273-283.

3. Avery, S.V., Microbial cell individuality and the underlying sources of heterogeneity. Nature Reviews Microbiology, 2006. 4(8): p. 577-587.

4. Cerf, O., Tailing of survival curves of bacterial spores. J ournal of Applied Microbiology, 1977. 42(1): p. 1-19.

5. Van Boeijen, I.K.H., C. Francke, R. Moezelaar, T. Abee, and M.H. Zwietering, Isolation of highly heat-resistant Listeria monocytogenes variants by use of a kinetic modeling-based sampling scheme. Applied and Environmental Microbiology, 2011. 77(8): p. 2617-2624.

6. Van Boeijen, I.K.H., R. Moezelaar, T. Abee, and M.H. Zwietering, Inactivation kinetics of three Listeria monocytogenes strains under high hydrostatic pressure. J ournal of Food Protection, 2008. 71(10): p. 2007-2013.

7. Karatzas, K.A.G. and M.H.J. Bennik, Characterization of a Listeria monocytogenes Scott A isolate with high tolerance towards high hydrostatic pressure. Applied and Environmental Microbiology, 2002. 68(7): p. 3183-3189.

8. Van Boeijen, I.K.H., A.A.E. Chavaroche, W.B. Valderrama, R. Moezelaar, M.H. Zwietering, and T. Abee, Population diversity of Listeria monocytogenes LO28: Phenotypic and genotypic characterization of variants resistant to high hydrostatic pressure. Applied and Environmental Microbiology, 2010. 76(7): p. 2225-2233.

9. Cotter, P.D. and C. Hill, Surviving the acid test: Responses of Gram-positive bacteria to low pH. Microbiology and Molecular Biology Reviews, 2003. 67(3): p. 429-453.

10. Hill, C., Virulence or niche factors: What's in a name? Journal of Bacteriology, 2012. 194(21): p. 5725-5727.

11. Van Boeijen, I.K.H., P.G. Casey, C. Hill, R. Moezelaar, M.H. Zwietering, C.G.M. Gahan, and T. Abee, Virulence aspects of Listeria monocytogenes LO28 high pressure-resistant variants. Microbial Pathogenesis, 2013. 59-60: p. 48-51.

12. Cotter, P.D., C.G.M. Gahan, and C. Hill, A glutamate decarboxylase system protects Listeria monocytogenes in gastric fluid. Molecular Microbiology, 2001. 40(2): p. 465-475.

13. Cotter, P.D., K. O'Reilly, and C. Hill, Role of the glutamate decarboxylase acid resistance system in the survival of Listeria monocytogenes LO28 in low pH foods. J ournal of Food Protection, 2001. 64(9): p. 1362-1368.

14. Davis, M.J., P.J . Coote, and C.P. O’Byrne, Acid tolerance in Listeria monocytogenes: The adaptive acid tolerance response (ATR) and growth-phase-dependent acid resistance. Microbiology, 1996. 142(10): p. 2975-2982.

15. Van der Veen, S. and T. Abee, Contribution of Listeria monocytogenes RecA to acid and bile survival and invasion of human intestinal Caco-2 cells. International J ournal of Medical Microbiology, 2011. 301(4): p. 334-340.

16. O'Driscoll, B., C.G.M. Gahan, and C. Hill, Adaptive acid tolerance response in Listeria monocytogenes: Isolation of an acid-tolerant mutant which demonstrates increased virulence. Applied and Environmental Microbiology, 1996. 62(5): p. 1693-1698.

17. Geeraerd, A.H., V. Valdramidis, and J.F. Van Impe, GInaFiT, a freeware tool to assess nonlog-linear microbial survivor curves. International J ournal of Food Microbiology, 2005. 102(1): p. 95-105.

18. Geeraerd, A.H., V.P. Valdramidis, and J.F. Van Impe, Erratum to "GInaFiT, a freeware tool to assess non-log-linear microbial survivor curves" (vol 102, pg 95, 2005). International J ournal of Food Microbiology, 2006. 110(3): p. 297-297. 
19. Den Besten, H.M.W., M. Mataragas, R. Moezelaar, T. Abee, and M.H. Zwietering, Quantification of the effects of salt stress and physiological state on thermotolerance of Bacillus cereus ATCC 10987 and ATCC 14579. Applied and Environmental Microbiology, 2006. 72(9): p. 5884-5894.

20. Mafart, P., O. Couvert, S. Gaillard, and I. Leguerinel, On calculating sterility in thermal preservation methods: Application of the Weibull frequency distribution model. International J ournal of Food Microbiology, 2002. 72(1-2): p. 107-113.

21. Belloch, C., M.C. Gurrea, A. Tárrega, F. Sampedro, and J.V. Carbonell, Inactivation of microorganisms in orange juice by high-pressure homogenization combined with its inherent heating effect. European Food Research and Technology, 2012. 234(5): p. 753-760.

22. Roth, S., J . Feichtinger, and C. Hertel, Characterization of Bacillus subtilis spore inactivation in low-pressure, low-temperature gas plasma sterilization processes. J ournal of Applied Microbiology, 2010. 108(2): p. 521-531.

23. Salomão, B.C.M., J J . Churey, G.M.F. Aragão, and R.W. Worobo, Modeling Penicillium expansum resistance to thermal and chlorine treatments. J ournal of Food Protection, 2009. 72(12): p. 2618-2622.

24. Browne, N. and B.C.A. Dowds, Acid stress in the food pathogen Bacillus cereus. J ournal of Applied Microbiology, 2002. 92(3): p. 404-414.

25. Veening, J.W., W.K. Smits, and O.P. Kuipers, Bistability, epigenetics, and bet-hedging in bacteria. Annual Review of Microbiology, 2008. 62: p. 193-210.

26. Lianou, A., J.D. Stopforth, Y. Yoon, M. Wiedmann, and J.N. Sofos, Growth and stress resistance variation in culture broth among Listeria monocytogenes strains of various serotypes and origins. J ournal of Food Protection, 2006. 69(11): p. 2640-2647.

27. Dykes, G.A. and S.M. Moorhead, Survival of osmotic and acid stress by Listeria monocytogenes strains of clinical or meat origin. International J ournal of Food Microbiology, 2000. 56(2- 3): p. 161166.

28. Skandamis, P.N., Y. Yoon, J.D. Stopforth, P.A. Kendall, and J.N. Sofos, Heat and acid tolerance of Listeria monocytogenes after exposure to single and multiple sublethal stresses. Food Microbiology, 2008. 25(2): p. 294-303. 


\section{3}

Diversity of acid stress resistant variants of Listeria monocytogenes and the potential role of ribosomal protein $\mathrm{S} 21$ encoded by rpsU

K.I. Metselaar, H.M.W. den Besten, J. Boekhorst, S.A.F.T. van Hijum, M.H. Zwietering and T. Abee

Published in:

Frontiers in Microbiology (2015) 6:422. 


\section{Abstract}

The dynamic response of microorganisms to environmental conditions depends on the behavior of individual cells within the population. Adverse environments can select for stable stress resistant subpopulations. In this study, we aimed to get more insight in the diversity within Listeria monocytogenes $\mathrm{LO} 28$ populations, and the genetic basis for the increased resistance of stable resistant fractions isolated after acid exposure. Phenotypic cluster analysis of 23 variants resulted in three clusters and four individual variants and revealed multiple-stress resistance, with both unique and overlapping features related to stress resistance, growth, motility, biofilm formation and virulence indicators. A higher glutamate decarboxylase (GAD) activity correlated with increased acid resistance. Whole genome sequencing revealed mutations in rpsU, encoding ribosomal protein S21 in the largest phenotypic cluster, while mutations in ctsR, which were previously shown to be responsible for increased resistance of heat and high hydrostatic pressure (HHP) resistant variants, were not found in the acid resistant variants. This underlined that large population diversity exists within one L. monocytogenes strain and that different adverse conditions drive selection for different variants. The finding that acid stress selects for rpsU variants provides potential insights in the mechanisms underlying population diversity of $\mathrm{L}$. monocytogenes. 


\section{Introduction}

Listeria monocytogenes is a ubiquitous microorganism, which may encounter a variety of environmental conditions during its transmission from soil to the human gastro-intestinal tract [1]. In the last decades, many researchers have demonstrated that microbial populations are not isogenic but comprise phenotypic and genotypic heterogeneity. The presence of subpopulations, each of which is prepared to survive and multiply under a specific condition allows the organism to survive in a wide range of environmental conditions. Van Boeijen et al. [2] showed that a set of HHP resistant variants of L. monocytogenes LO28 had different phenotypic and genotypic characteristics. The variants showed highly increased resistance towards multiple types of stress, different degrees of motility and different growth rates. Despite the fact that the presence of stable resistant subpopulations has been clearly demonstrated not only for L. monocytogenes [3, 4] but also for other microorganisms $[5,6]$, the mechanisms behind the increased stress resistance of these subpopulations are poorly understood. A mutation in the class III heat shock repressor ctsR was shown to be responsible for the increased HHP resistant phenotype for some L. monocytogenes variants $[2,7,8]$. Mutations in ctsR can lead to a defect in the repression of a number of chaperone encoding genes like clpC which results in transcription of these stress response genes. ctsR variants have been isolated after HHP stress in several L. monocytogenes strains and is the only mutation identified in stress resistant variants until now. Interestingly, the HHP resistant variants also showed increased resistance to other types of stress, including heat and acid stress. This led to the hypothesis that also heat and acid stress could select for this type of variants. Both heat and acid resistant variants have been isolated recently $[8,9]$ and for most of these heat resistant variants a mutation in ctsR was found which appears to be responsible for its increased heat resistance. However, the acid resistant variants are not further characterized yet and it is not known if these variants harbour the same mutation or if acid stress selects for a different type of variants. One of the most important and well-studied systems in acid resistance of L. monocytogenes is the glutamate decarboxylase (GAD) system [10]. An extracellular glutamate molecule is imported by an antiporter in exchange for an intracellular $\gamma$-aminobutyrate (GABA) molecule. Each molecule of glutamate is decarboxylated by a decarboxylase to produce a molecule of GABA. During this process a proton is consumed. This results in an increase of the cytoplasmic $\mathrm{pH}$ and thus protects the cell against the acidic environmental conditions [11]. Recently, a new model for the GAD system has been proposed by Karatzas et al. [12], in which two different GAD systems were discriminated. The intracellular GAD system uses metabolically synthesized glutamate and the extracellular system uses environmental glutamate 
which is accumulated in the cell by dedicated transporters. The intracellular system for the production of GABA seems to be important for the LO28 strain in Brain Heart Infusion (BHI), which we also used in our study. Our study aims to get more insight in the population diversity of L. monocytogenes $\mathrm{LO} 28$ and the mechanisms underlying the multiple stress resistance. The combined phenotypic and genotypic approach led to new insights in the mechanisms of increased stress resistance of $\mathrm{L}$. monocytogenes $\mathrm{LO} 28$ variants. Our findings emphasize the genotypic diversity within the L. monocytogenes population and indicate that different types of stress lead to the selection for different types of multiple stress resistant variants.

\section{Materials and Methods}

\section{Bacterial strains and culturing conditions}

Listeria monocytogenes LO28 wild type (WT) (Wageningen UR Food \& Biobased Research, the Netherlands) and acid resistant variants [9] were used in this study. The stock culture was kept in $15 \%$ (v/v) glycerol (Fluka, Buchs) at $-80^{\circ} \mathrm{C}$, and before the experiments cells from stock were grown for 2 days at $30^{\circ} \mathrm{C}$ on brain heart infusion (BHI) agar (Oxoid, Hampshire). A single colony was used to inoculate $20 \mathrm{ml}$ BHI broth. After overnight (ON) growth $\left(18-22 \mathrm{~h}\right.$ ) at $30^{\circ} \mathrm{C}$ (Innova 4335; New Brunswick Scientific, Edison, NJ) with shaking at $160 \mathrm{rpm}, 0.5 \%(\mathrm{v} / \mathrm{v})$ inoculum was added to fresh $\mathrm{BHI}$ broth. Cells were grown in $\mathrm{BHI}$ at $30^{\circ} \mathrm{C}$ until late-exponential growth phase $\left(\mathrm{OD}_{600}=0.4-0.5\right.$ reached after 4-5 h) or stationary phase (18-22 h of growth, $\mathrm{OD}_{600}$ 2.0). Independent triplicates were performed for each experiment, unless indicated otherwise. For stress experiments, plates were incubated for $4-6$ days at $30^{\circ} \mathrm{C}$ to allow recovery of the cells. Results were expressed as reduction in $\log _{10} \mathrm{cfu} / \mathrm{ml}$ by taking the difference in $\log _{10} \mathrm{cfu} / \mathrm{ml}$ at $\mathrm{t}_{0}$ and $\mathrm{t}_{\mathrm{x}}$. For all other experiments, plates were incubated for 2 days at $30^{\circ} \mathrm{C}$.

\section{Growth characteristics}

The grouth rate at $37^{\circ} \mathrm{C}$ and $7^{\circ} \mathrm{C}$ was determined by the 2 -fold dilution (2FD) method as described by Biesta-Peters et al. [13]. Briefly, stationary phase cultures were used for this experiment and diluted in BHI broth to an initial concentration of $\sim 5 \cdot 10^{5}$ $\mathrm{cfu} / \mathrm{ml}$ which was confirmed by plating on BHI agar plates. From this culture, four 2FD in BHI broth were made in duplicate in a 100 well honeycomb plate and the final volume in each well was $200 \mu$. The plate was incubated in the Bioscreen C (Oy Growth Curves AB Ltd, Helsinki, Finland). The Bioscreen C was set to $37^{\circ} \mathrm{C}$ or $7^{\circ} \mathrm{C}$ with medium shaking and the $\mathrm{OD}_{600}$ was measured every 10 or 30 min respectively. 
For the $7^{\circ} \mathrm{C}$ measurements, the Bioscreen $\mathrm{C}$ was placed in a cold room with an ambient temperature of $\sim 4^{\circ} \mathrm{C}$. This was done up to 3 weeks, until all wells reached an $\mathrm{OD}_{600}$ of at least 0.2 (time to detection (TTD). The maximum specific growth rate was determined by taking the reciprocal of the slope between the TTD and the $\ln \left(\mathrm{N}_{0}\right)$ for the four two-fold dilutions. These experiments were done with biological duplicates $\left(7^{\circ} \mathrm{C}\right)$ and triplicates $\left(37^{\circ} \mathrm{C}\right)$.

\section{Heat resistance}

Heat inactivation experiments were carried out at $55 \pm 0.3^{\circ} \mathrm{C}$ in a water bath with shaking at $160 \mathrm{rpm}$ (Julabo SW 23, Julabo Labortechnik, Germany). 400 pl lateexponential phase culture was transferred into $40 \mathrm{ml}$ preheated BHI broth. After 6 min, $1 \mathrm{ml}$ was taken and decimally diluted in peptone physiological salt (PPS; $0.1 \%$ peptone and $0.85 \% \mathrm{NaCl}$ ). A separate Erlenmeyer containing $\mathrm{BHI}$ broth at room temperature was used for the $\mathrm{t}=0$ measurement. Appropriate dilutions were spiral plated on BHI agar (Eddy J et, IUL Instruments).

\section{Hydrogen peroxide resistance}

Late-exponential phase cells were exposed to $420 \mathrm{mMH}_{2} \mathrm{O}_{2}$ for 9 min. This time point was based on the inactivation kinetics of the WT and selected from the data points representing the fast inactivation phase preceding tailing, to achieve a significant reduction in plate counts but with final numbers well above the detection limit. The colony count at $\mathrm{t}=0 \mathrm{~min}$ was determined by adding directly $1 \mathrm{ml}$ of thelate-exponentialculture into $9 \mathrm{ml}$ PPS. The appropriate amount of $30 \%$ (w/v) $\mathrm{H}_{2} \mathrm{O}_{2}$ was added to the late-exponential phase culture and incubated at $30^{\circ} \mathrm{C}$ in a shaking waterbath. After 9 min $1 \mathrm{ml}$ was taken, added to $9 \mathrm{ml}$ PPS and appropriate dilutions were made. Diluting and plating were done immediately to avoid continuous $\mathrm{H}_{2} \mathrm{O}_{2}$ inactivation in PPS.

\section{Benzalkonium chloride resistance}

Resistance of late-exponential phase cells to benzalkonium chloride (BAC) was determined similarly as described for hydrogen peroxide. Prior to the experiment, the count at $\mathrm{t}=0$ was determined by serial dilution of the late-exponential phase culture in PPS. Then, $200 \mu \mathrm{l}$ of a $5 \mathrm{~g} / \mathrm{L}$ BAC stock solution was added to $50 \mathrm{ml}$ late-exponential phase culture, resulting in a $20 \mathrm{mg} / \mathrm{L}$ final concentration. The culture was incubated at $30^{\circ} \mathrm{C}$ in a waterbath shaking at $160 \mathrm{rpm}$ and after $5 \mathrm{~min}$, a sample was taken and decimally diluted in PPS. Appropriate dilutions were spiral plated on BHI agar. 


\section{Glutamate decarboxylase activity}

Glutamate decarboxylase activity was tested for both late-exponential phase cells and stationary phase cells. $100 \mathrm{ml}$ culture was used, of which $50 \mathrm{ml}$ was used for $\mathrm{t}=0$ measurements and $50 \mathrm{ml}$ for measurements after exposure to $\mathrm{pH} 4.0$ for 60 minutes. This was chosen since no inactivation was observed for this conditions (data not shown) but it is described by Karatzas et al. [12] that the GAD system is activated under these conditions. Cells were harvested by centrifugation at $7000 \mathrm{x} \mathrm{g}$ and were resuspended in either BHI adjusted to $\mathrm{pH} 4.0$ by $10 \mathrm{M} \mathrm{HCl}$ (acid exposed samples) or $\mathrm{BHI}$ ( $\mathrm{t}=0$ samples). GABA concentration was determined on cell lysates of $\mathrm{t}=0$ and acid exposed samples and $\mathrm{GABA}_{\mathrm{e}}$ concentration was determined in the medium of the acid exposed cells. Cell lysates were prepared as follows: $50 \mathrm{ml}$ culture was harvested by centrifugation at $9000 \mathrm{x} \mathrm{g}$. The cell pellet was resuspended in $1 \mathrm{ml}$ BHI, resulting in a cell count of $\sim 10^{11} \mathrm{cells} / \mathrm{ml}$. Cell suspensions (or media) were boiled for 10 minutes in an oil bath to lyse all the cells. Subsequently, the cell lysates or media were centrifuged in a tabletop centrifuge for 10 minutes at 9503 $\mathrm{x}$ g. 500 $\mathrm{ll}$ of the supernatant was transferred to a clean tube and used for GABA determination. A commercial preparation known as GABase (Sigma-Aldrich, the Netherlands) was used to determine the $\mathrm{GABA}_{i}$ and $\mathrm{GABA}_{\mathrm{e}}$ concentrations. GABA was quantified as described by Tsukatani et al. [14] with modifications of Karatzas et al. [15] and O'Byrne et al. [16]. To correct for possible differences in cell density and cell size, the GABA results were corrected for total protein content. Total protein determination was done by using the Pierce BCA Protein Assay Kit (Thermo Scientific, the Netherlands). Total protein was extracted using B-PER ${ }^{\mathrm{TM}}$ Bacterial Protein Extraction Reagent (Thermo Scientific, the Netherlands) according to the manufacturer's instructions.

\section{Motility testing}

The motility of the strains was tested as described previously [2]. Briefly, semisolid medium containing $0.25 \%$ (wt/vol) agar (Oxoid), $1 \%$ (wt/vol) bacteriological peptone (Oxoid), 0.5\% (wt/vol) NaCl (Merck), 0.005\% (wt/vol) 2,3,5-triphenyltetrazolium chloride (Sigma-Aldrich, the Netherlands) was inoculated by stabbing a single colony into the medium. After 3 days of incubation at $30^{\circ} \mathrm{C}$, motile strains showed a red cloudy pattern as a result of the reduction of 2,3,5- triphenyltetrazolium chloride to formazan caused by bacterial metabolism. The LO28 WT and immotile HHP variant 17 [2] were used as positive and negative control respectively. Isolates which showed no or reduced motility at $30^{\circ} \mathrm{C}$ were retested at $25^{\circ} \mathrm{C}$ to confirm reduced motility, since it is know that motility is temperature dependent [17]. 


\section{Biofilm formation}

Biofilm formation was studied in $\mathrm{BHI}$ at $30^{\circ} \mathrm{C}$ under static conditions. ON cultures were inoculated in fresh $\mathrm{BHI}$ broth $(0.5 \% \mathrm{v} / \mathrm{v})$ and a polystyrene 12-well plate was filled with $3 \mathrm{ml}$ of this cell culture in duplicate. The polystyrene plates were incubated at $30^{\circ} \mathrm{C}$ for $48 \pm 2 \mathrm{~h}$. After incubation, the supernatant was removed and the biofilms were washed 3 times with phosphate buffered saline (PBS). Biofilms were resuspended in $1 \mathrm{ml}$ PBS by rigorous pipetting, serially diluted in PBS and plated on BHI agar. Plates were incubated at $30^{\circ} \mathrm{C}$ and counted after 2 days.

\section{Virulence indicators}

Lysis of the vacuole and thereby escape from the phagosomes, is critical in L. monocytogenes virulence [18]. Phosphatidylcholine phospholipase C (PC-PLC), or lechitinase, production is necessary for the breakdown of the bacterial plasma membrane after invasion in a human host cell, and is therefore considered a virulence factor in L. monocytogenes [19]. PC-PLC activity was measured as described by Coffey et al. [20]. Briefly, $2 \mu \mathrm{ON}$ culture was spotted in triplicate on BHI agar plates, containing 3\% $\mathrm{NaCl}(\mathrm{w} / \mathrm{v})$ and $5 \%(\mathrm{v} / \mathrm{v})$ egg yolk. Plates were incubated for 2 days at $37^{\circ} \mathrm{C}$ and PC-PLC activity was determined by evaluating the precipitation zone around the colonies. The second factor which is responsible for vacuole escape is the pore-forming hemolysin, Listeriolysin O (LLO) [18]. LLO activity can be measured by degradation of red blood cells. Hemolysis was tested according to the ISO 112901 method. Single colonies were streaked on blood agar plates containing $6 \%$ sheep blood. Plates were examined after 24 hours of incubation at $37^{\circ} \mathrm{C}$ and marked either ++ (clear light zone of hemolysis), + (clear light zone under the colony, but not around) or - (no clear zone) for hemolysis.

\section{gDNA extraction}

Genomic DNA of L. monocytogenes ON cultures was isolated by GenElute Bacterial Genomic DNA Kit (Sigma-Aldrich) using the manufacturers protocol for Grampositives. Instead of the supplied Elution Solution, $10 \mathrm{mM}$ Tris- $\mathrm{HCl} \mathrm{pH} 8.5$ was used for the elution step to avoid residues of EDTA which can interfere with the sequencing reaction. gDNA concentration and purity was measured with an Eppendorf BioPhotometer. gDNA was stored at $-20^{\circ} \mathrm{C}$ until further use in amplicon sequencing and whole genome sequencing. 


\section{Amplification and sequencing of cts $R$ and $r p s U$}

Primers used for amplification of ctsR [3] and rpsU are listed in Table 3.1. Primers used for the amplification were designed on the genome of L. monocytogenes EGDe by Primer3 and checked to also work for LO28. The amplification was performed with REDTaq DNA Polymerase (Sigma-Aldrich), at an annealing temperature of $55^{\circ} \mathrm{C}$ and with an elongation time of $80 \mathrm{~s}$ in a MyCycler PCR instrument (Bio-Rad, the Netherlands). For variant 14 , for which no rpsU PCR product was found, another primer pair was designed (rpsU_ 14, Table 3.1). The PCR products (1.2 kb and 900bp for ctsR and rpsU respectively) were purified by QIAquick PCR Purification Kit (Qiagen, the Netherlands) and sent for sequence analysis (Base Clear B.V., Leiden, the Netherlands). Sequences of the variants were compared to the WT sequence in BioEdit (v7.1.3).

Table 3.1: primers used for PCR followed by amplicon sequencing

\begin{tabular}{|c|c|c|c|}
\hline \multicolumn{4}{|c|}{ Primers used for PCR followed by amplicon sequencing } \\
\hline target & forward primer $\left(5^{\prime} \rightarrow 3^{\prime}\right)$ & reverse primer $\left(5^{\prime} \rightarrow 3^{\prime}\right)$ & source \\
\hline$c t s R$ & GCAGGGATAAACGCTGAAAG & ACTCCGGACATCCAACTC & [3] \\
\hline rpsU & CGCGTAGTCCTCCACAATGA & GCCAGAGAAGGCGAGGATAG & This study \\
\hline$r p s U_{-} 14$ & ACGATTTCATGTTGACGATGC & CGGTCCATTAGCCTTGTTGT & This study \\
\hline \multicolumn{4}{|c|}{ Primers used for RT-PCR } \\
\hline target & forward primer $\left(5^{\prime} \rightarrow 3^{\prime}\right)$ & reverse primer $\left(5^{\prime} \rightarrow 3^{\prime}\right)$ & source \\
\hline $16 S$ & GATGCATAGCCGACCTGAGA & TGCTCCGTCAGACTTTCGTC & [2] \\
\hline tpi & AACACGGCATGACACCAATC & CACGGATTTGACCACGTACC & [2] \\
\hline$c t s R$ & GATTAATGGTTGCGGCATTG & CAAAGCAACTAACATCGCTCT & [2] \\
\hline$c / p C$ & AGTCGATGTTTGGCGATGAG & TGGAGGAGCCCCAACTAAAC & [2] \\
\hline $\operatorname{sig} B$ & GAAGCAATGGAAATGGGAAA & CATCATCCGTACCACCAACA & This study \\
\hline $\operatorname{gadD2}$ & AATACCTTGCCCATGCAGTC & GGCTTGGAAATCTTGGATGA & {$[15]$} \\
\hline $\operatorname{gadT2}$ & CACGGCTAAAATCGCAAAAT & GGAAGCTTCAACAAAAATGT & {$[15]$} \\
\hline$r p s U$ & CGTTTAAAGCGACGAAGAGCA & CGGAGGGAGGGAAAGAGAGA & This study \\
\hline
\end{tabular}

\section{Gene expression of target genes}

Gene-expression was studied in late-exponential phase. $1 \mathrm{ml}$ cell culture was harvested by centrifugation at $13684 \mathrm{x} \mathrm{g}$ (Heraeus Biofuge Pico). The cell pellet was resuspended in $1 \mathrm{ml}$ TRI reagent (Applied Biosystems) and flash frozen in liquid nitrogen. Samples were stored at $-80^{\circ} \mathrm{C}$ until RNA extraction. RNA extraction was performed using the Direct-zol RNA MiniPrep kit (Zymo Research) according to 
the manufacturer's instructions, including the DNase treatment. RNA quantity was measured on the Nanodrop and the RNA was checked on degradation by gel electrophoresis. RNA samples were stored at $-80^{\circ} \mathrm{C}$. cDNA synthesis was performed by SuperScript III reverse transcriptase (Invitrogen). The real-time PCR was performed using SYBRgreen PCR MasterMix (Applied Biosystems) in a Bio-Rad CFX96 RT-PCR machine. The following steps were performed: initial denaturation (4 min at $95^{\circ} \mathrm{C}$ ), amplification ( 40 cycles of $95^{\circ} \mathrm{C}$ for $15 \mathrm{~s}, 59^{\circ} \mathrm{C}$ for $1 \mathrm{~min}$ ) and a melting step $\left(65-95^{\circ} \mathrm{C}\right.$ with $0.5^{\circ} \mathrm{C}$ steps) to confirm a single product was formed. A standard curve was included to calculate the PCR efficiency for each primer pair. Primers used in this study were taken from literature or designed by Primer3 and are listed in Table 3.1. 16S and tpi were used as reference genes and evaluated by BestKeeper (version 1) [21]. Relative expression ratios were calculated with the pairwise fixed reallocation randomization test using the relative expression software tool (REST) [22].

\section{Whole genome sequencing and structural variation (SV) analysis}

Genomic DNA of LO28 WT and variants 3, 7, 9, 10, 12, 14, 15, 17, 21 and 22 was sent to GATC Biotech (Germany) for library preparation and Illumina paired end sequencing. The WT reads were assembled using the Ray assembler [23] and annotated with RAST [24]. For the SV (SNPs and small insertion and deletions) analysis, low-quality ends of reads (Phred score 540 ) were trimmed and reads shorter than 31 bases were removed with Trim Galore (http:/ / www.bioinformatics. babraham.ac.uk/projects/trim_galore/). SV analysis was done with the tool ROVAR (http://trac.nbic.nl/rovar), which uses BLAST-like alignment tool (BLAT) [25] version 34, to align reads to a repeat-masked reference sequence. A stringent filtering approach was used to minimize false-positive SVs calls: (1) a SNP should be supported by at least 1 forward read and 1 reverse read, (2) by at least 5 unique reads, (3) by at least 20 reads in total, and (4) at least $99 \%$ of the reads mapping to the region of the SNP should support the SNP.

\section{Clustering of variants based on phenotype}

Initial clustering was performed with the WT and a set of 7 selected variants (3, 7, 9, 13, 14, 15 and 23) for which a total of 14 phenotypes were determined. The phenotypes that were included in the clustering are: acid- , heat- , $\mathrm{BAC}$ and $\mathrm{H}_{2} \mathrm{O}_{2}$-resistance of late-exponential phase cells, maximum specific growth rate in $\mathrm{BHI}$ at $7^{\circ} \mathrm{C}, 37^{\circ} \mathrm{C}$ (Figure 3.1 ), $30^{\circ} \mathrm{C}$ and in $\mathrm{pH} 5.0$ at $30^{\circ} \mathrm{C}$ [9], biofilm formation, motility, hemolysis and phospholipase activity (Table 3.2), GAD activity (Figure 3.3) and cell size (data not shown). Hierarchical clustering was performed in SPSS (IBM SPSS Statistics 19) by Euclidian distance and within group linkage. Values were standardized on 
z-values. Based on the 6 phenotypes that were determined for all 23 variants (growth at $7^{\circ} \mathrm{C}$ and $37^{\circ} \mathrm{C}$, acid resistance, motility, hemolysis and phospholipase activity) the remaining 16 variants were added to a matching cluster if possible.

\section{Statistical analysis}

A 2-tailed Student's t-test was used to determine statistically significant differences between the WT and variants for the phenotypes. A $\mathrm{p}<0.05$ was considered significant.

\section{Results}

\section{Growth characteristics and selection of model variants}

Twenty-threestableacidresistantvariants of L. monocytogenes LO28 were previously divided into 3 groups, based on their acid resistance: slightly acid resistant, highly acid resistant and variably acid resistant [9]. The maximum specific growth rate was determined for these 23 variants in $\mathrm{BHI}$ broth at $7^{\circ} \mathrm{C}$ and at $37^{\circ} \mathrm{C}$ (Figure 3.1). At $37^{\circ} \mathrm{C}$ variant 9 clearly grew slower than the wild type (WT). Also variants 12 and 13 showed a significantly reduced growth rate at $37^{\circ} \mathrm{C}$.

Variants 8 and 12, although displaying a significantly lower $\mu_{\max }$ than the WT $(0.053$ and 0.058 versus $0.076 \mathrm{~h}^{-1}$ respectively), did not show such a dramatically decreased growth rate The other variants showed similar growth as the WT at $37^{\circ} \mathrm{C}$ and the clear trend between acid resistance and growth rate which was established at $30^{\circ} \mathrm{C}$ [9] was not so apparent at $37^{\circ} \mathrm{C}$. However, at $7^{\circ} \mathrm{C}$ there was a clear reduction in growth rate for the most highly acid resistant variants. Only variants 10 and 13 of the highly resistant group did not show a significantly lower $\mu_{\max }$ than the WT, all the other highly resistant variants did. as the other variants from the highly resistant group $\left(\mu_{\max }<0.035 \mathrm{~h}^{-1}\right)$. Variant 7 showed a significantly reduced growth rate. Within the group of slightly resistant variants, only variant 5 had a significantly lower growth rate than the WT. The acid resistance and growth characteristics of the 23 variants were the basis for selecting seven model variants for further detailed phenotypic characterization, namely variants 3, 7, 9, 13, 14, 15 and 23. 

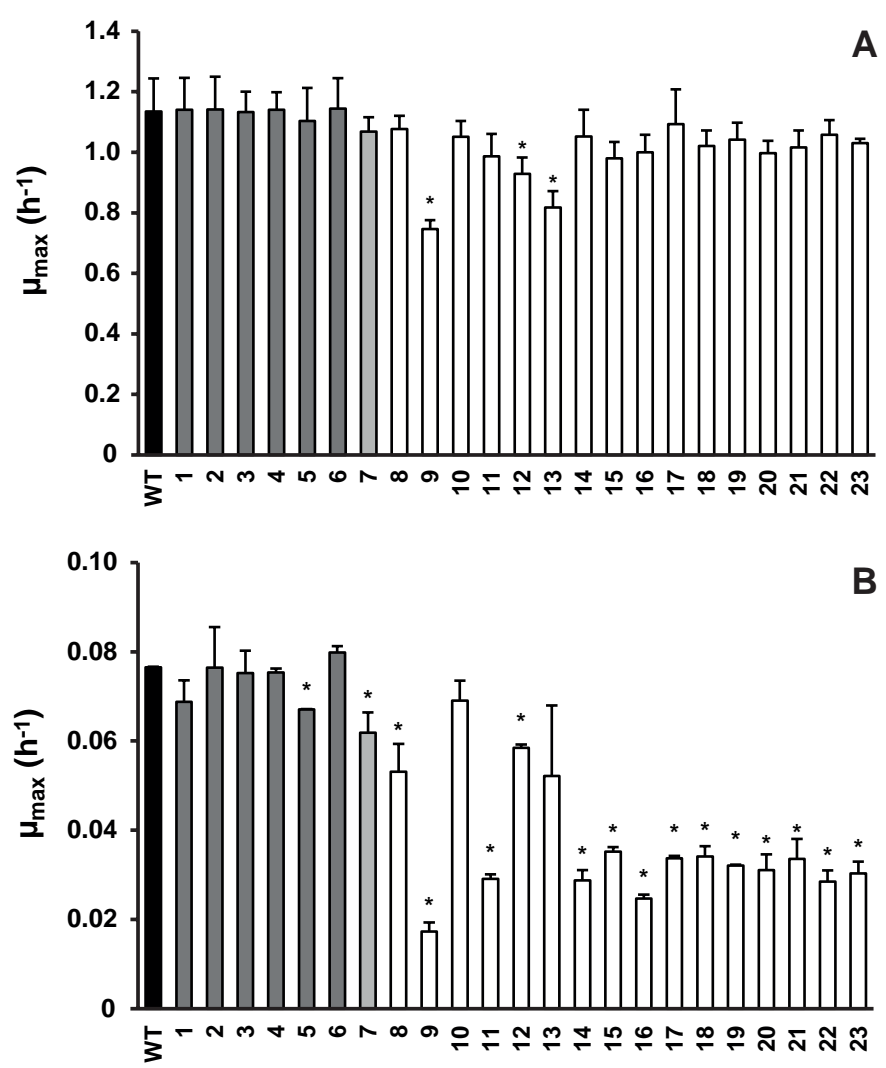

Figure 3.1: Maximum specific grouth rate of $\mathrm{L}$. monocytogenes $\mathrm{LO} 28 \mathrm{WT}$ and acid resistant variants in $\mathrm{BHI}$ at $37^{\circ} \mathrm{C}(\mathrm{A})$ and $7^{\circ} \mathrm{C}(\mathrm{B})$, determined by the 2 fold dilution method. Dark grey bars indicate the slightly resistant variants, light grey the variable resistant variant and white the highly acid resistant variants [9]. Error bars represent the standard deviation of the mean for three (A) or two (B) independent replicates. Significantly different values for $\mu_{\max }$ compared to the WT are indicated by $*(\mathrm{p}<0.05)$

\section{Multiple stress resistance and the role of the glutamate decarboxylase system}

Late-exponential-phase cells of the selected variants and the WT were exposed to heat, hydrogen peroxide and benzalkonium chloride (BAC). Heat and acid resistance were correlated in these variants (Figures 3.2A and 3.2B). The five variants from the highly acid resistant group also showed highly increased survival after 6 minutes exposure to $55^{\circ} \mathrm{C}$ compared to the WT. Whereas the WT shows an almost $4 \log _{10} \mathrm{cfu} /$ $\mathrm{ml}$ reduction after the exposure time of 6 minutes, there was less than $0.5 \log _{10} \mathrm{cfu} /$ $\mathrm{ml}$ reduction for these five variants. Variant 7 , showing variable acid resistance, was also significantly more heat resistant than the WT, but with $1 \log _{10}$ reduction less resistant than the highly resistant group. Slightly acid resistant variant 3 did not show significantly increased heat survival. Exposure to the disinfectants hydrogen 

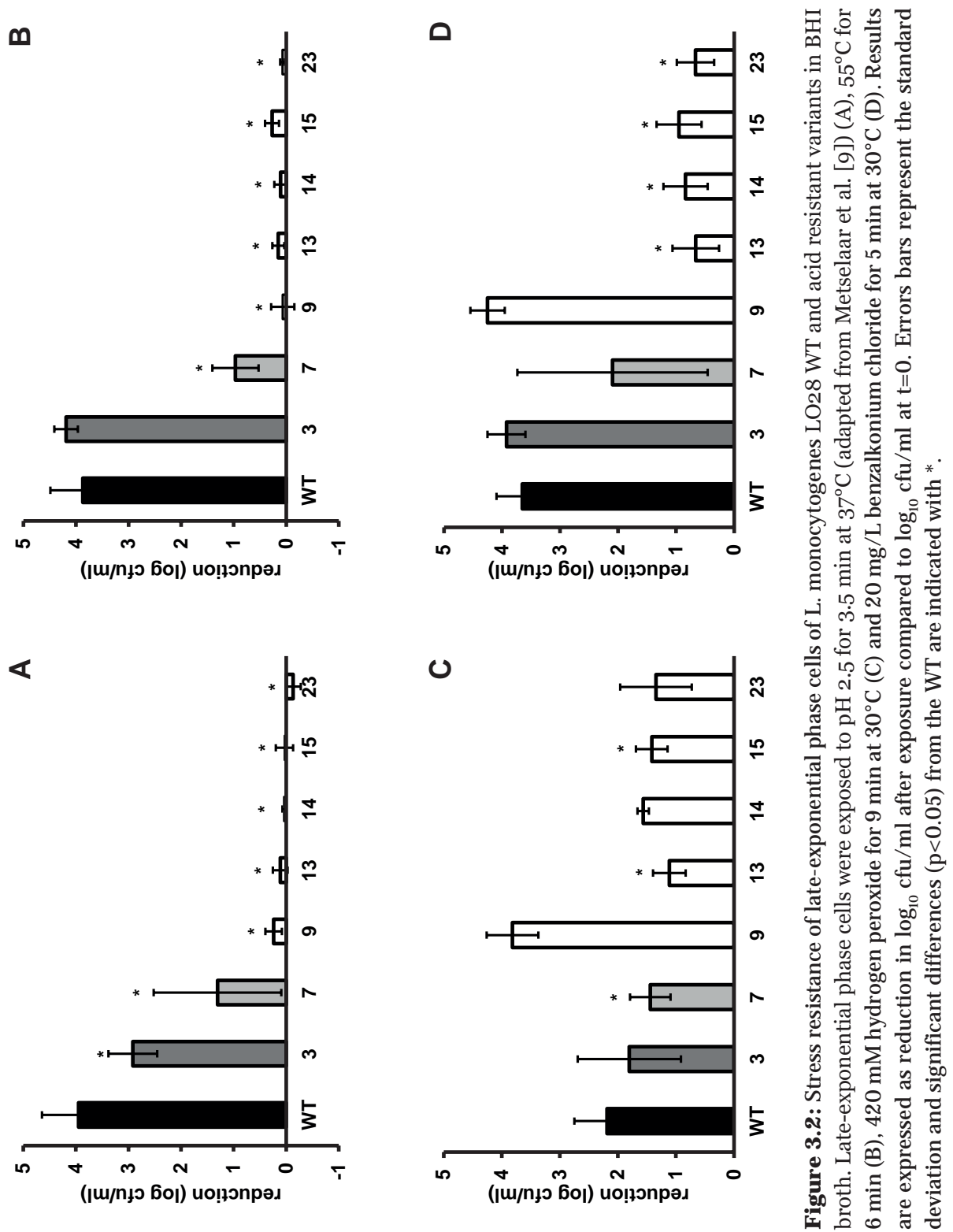

ष 1005

ญ

द्वि $\mathrm{m}$

त्త్ర त्ठ

屯류

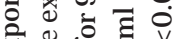

ख 4

들 잉 웅

0 으

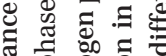

2 0

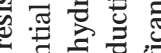

\& छ $\sum_{0}$

क क्षिठ ठठ

ले 范 है

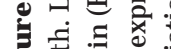
玨 0 잉 
peroxide and benzalkonium chloride showed a different trend (Figures $3.2 \mathrm{C}$ and 3.2D) and revealed diversity within the highly resistant group. Variant 9 showed a significantly increased sensitivity to $\mathrm{H}_{2} \mathrm{O}_{2}$ and a similar sensitivity as the WT towards BAC, whereas variants $13,14,15$ and 23 were significantly more resistant to BAC. Variant 7 showed a variable response towards BAC, as was also observed after acid exposure.

The GAD system is one of the most important systems in L. monocytogenes to overcome acid stress. Therefore, the activity of the GAD system in the WT and variants was evaluated, by measuring the amount of intracellular and extracellular GABA ( $\mathrm{GABA}_{\mathrm{i}}$ and $\mathrm{GABA}_{\mathrm{e}}$ respectively) before and after acid exposure. From Figure 3.3 it can be seen that the WT and all variants had a functional GAD system. It was also attempted to measure GABA levels, but in all cases the concentration was below the detection limit. In all cases the amount of GABA, was increased after an hour exposure to $\mathrm{pH} 4.0$. Variants 13, 14, 15 and 23 displayed higher levels of intracellular GABA, both before and after exposure to $\mathrm{pH} 4.0$ compared to the WT. Variant 7 showed a significantly higher GAD activity before acid exposure but was not significantly different ( $\mathrm{p}=0.052$ ) from the WT after $\mathrm{pH} 4.0$ exposure. Especially when comparing the GABA levels after acid exposure, it can be seen that variants 14 , 15 and 23 showed more than twice the GABA levels as those measured in the WT.

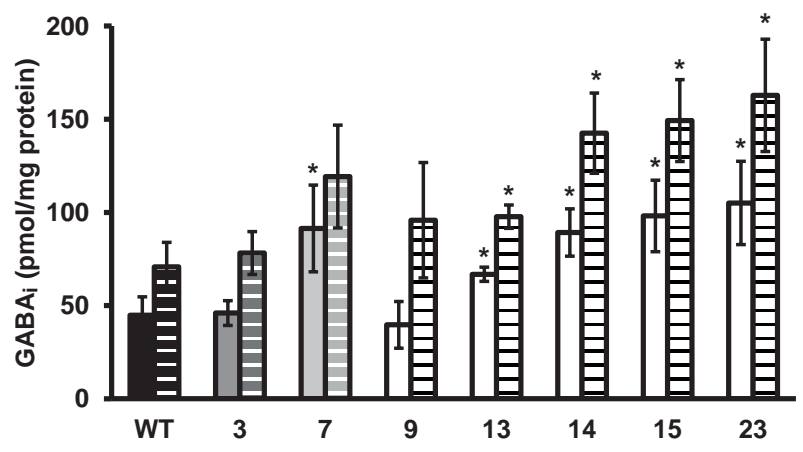

Figure 3.3: GABA concentration of stationary phase cells exposed to $\mathrm{pH} 4.0 \mathrm{in} \mathrm{BHI}$ for $60 \mathrm{~min}$. Solid bars are the $\mathrm{GABA}_{1}$ concentrations at $\mathrm{t}=0$ and dashed bars at $\mathrm{t}=60 \mathrm{~min}$. $\mathrm{GABA}_{1}$ concentrations are corrected for total amount of protein. Error bars include the standard deviation of the GABA and protein measurements and significant differences $(\mathrm{p}<0.05)$ from the WT are indicated with*

This indicated a possible role of the GAD system in the observed increased acid resistance. However, the increased acid resistance observed in variant 9 was not reflected by an increased GAD activity. On the other hand, the relative increase in GABA concentration after one hour exposure to $\mathrm{pH} 4.0$ compared to $\mathrm{t}=0$ was higher for this variant. The expression levels of selected GAD genes gadD2 and gadT2 in 
stationary-phase cells grown in BHI were determined for the WT and variants 3, 7, 9, 13, 14 and 23 by RT-PCR. These genes were chosen as they have been shown to be the major contributors in survival at severe $\mathrm{pH}$ stress [26]. No significant differences in the expression of these genes between WT and variants was observed.

\section{Motility, biofilm formation and virulence indicators}

Motility was tested for all 23 acid resistant variants and all variants were motile at $25^{\circ} \mathrm{C}$ and $30^{\circ} \mathrm{C}$ (Table 3.2) suggesting that all the variants possessed flagella, although variants 10 (data not shown) and 13 showed somewhat reduced motility. Some HHP resistant variants lost their motility completely [2], which was apparently not the case for the currently investigated variants. Biofilm formation was tested for the seven selected variants in $\mathrm{BHI}$ at $30^{\circ} \mathrm{C} .48 \mathrm{~h}$ biofilms were evaluated based on their viable counts (Table 3.2). All variants were capable of forming dense biofilms, with counts higher than $9 \log _{10} \mathrm{cfu} /$ well for all variants, and these counts were comparable to the WT. All the variants and the WT were positive for the virulence indicators hemolysis and phospholipase-C. However, both indicators were reduced in variant 13 compared to the WT (Table 3.2).

Table 3.2: Motility, biofilm formation, hemolysis, phospholipase-C activity of the variants

\begin{tabular}{|lllll|}
\hline & Motility & Biofilm formation & Phospholipase-C & Hemolysis \\
\hline LO28 WT & ++ & ++ & ++ & ++ \\
Variant 3 & ++ & ++ & ++ & ++ \\
Variant 7 & ++ & ++ & ++ & ++ \\
Variant 9 & ++ & ++ & ++ & ++ \\
Variant 13 & + & ++ & + & + \\
Variant 14 & ++ & ++ & ++ & ++ \\
Variant 15 & ++ & ++ & ++ & ++ \\
Variant 23 & ++ & ++ & ++ & ++ \\
\hline
\end{tabular}

For motility, biofilm formation and hemolysis: ++: strongly positive, +: positive. For phospholipase-C: +++: strongly positive, ++: positive, +: positive but weak.

\section{Evaluation of the genetic basis for increased stress resistance}

The ctsR and upstream region was sequenced for all the 23 variants. HHP resistant variant 6 (HHP6) [2] was included as a control, since this variant is known to have a GGT deletion in the glycine repeat region. The 3 bp deletion was confirmed in this variant. None of the acid resistant variants had a mutation in the sequenced ctsR 
region (data not shown). The sequencing data were confirmed by expression data of clpC and ctsR of late-exponential-phase cells. None of the seven model variants showed a significant up or down regulation of these genes compared to the WT (data not shown). This is in contrast to the observed upregulation of both ctsR and clpC of the HHP6 ctsR variant [2]. Both the sequencing and expression data showed that the increased resistance of the acid resistant variants is not caused by a ctsR mutation. Whole genome sequencing of 10 acid resistant variants $(3,7,9,10,12,14,15,17$, 21 and 22) and LO28 WT, followed by SV analysis indicated 3 single nucleotide polymorphisms (SNPs) in a total of 5 variants. Variant 3 and 17 had a T instead of a $\mathrm{G}$ at position 2933571. Due to the low quality of the sequence in this region it was not clear whether this SNP was present in an open reading frame (ORF) and therefore not further looked into. Variant 12 had a T instead of a C at position 1411623, which is located in a hypothetical protein. This SNP was confirmed in variant 12 by PCR and amplicon sequencing. This gene of the other variants was also amplified and sequenced, but found to be intact in all 22 other variants. The third SNP was the same mutation (C instead of $\mathrm{G}$ at position 1652445) in variant 15, 17 and 21. A nucleotide BLAST search of the ORF where this presumable SNP was present indicated that the annotated gene was rpsU, encoding $30 \mathrm{~S}$ ribosomal protein S21. Amplification of rpsU and its promoter region of all 23 variants, followed by sequencing of the resulting PCR products, confirmed an rpsU mutation to be present in these 3 and an additional 8 variants. The mutations are visualized in Figure 3.4.

Although all 11 variants had a mutation in the same region, 5 different mutations were identified amongst them. Variants 11, 15, 17, 20 and 21 had the same point mutation, which resulted in an arginine changing into a proline in the protein. Variant 23 had a point mutation in the ribosome binding site (RBS) of rpsU. Variants 16, 19 and 22 had a $4 \mathrm{bp}$ deletion, also in the RBS of rpsU. Variant 18 had $1 \mathrm{bp}$ deleted in the rpsU gene, resulting in a frameshift after 8 amino acids. Lastly, variant 14 did not give a PCR product with therpsU primers used (Table 3.1). Another primer pair (rpsU_14), aiming ata $1.6 \mathrm{~kb}$ product for theWT, resulted in a PCR product of $\sim 300 \mathrm{bp}$ for variant 14. A 1346 bp deletion, starting 144 bp upstream rpsU and covering rpsU, yqeY and half of phoH was confirmed in this variant after amplicon sequencing. Although different mutations appeared to be present, all these changes in nucleotide sequence affect the RBS or amino acid sequence in such a way that they potentially resulted in a reduction of active S21 protein. Expression of rpsU during late-exponential phase was determined for the seven model variants and confirmed that in the rpsU variants 14,15 and 23, expression of rpsU was significantly downregulated by $5.8 \log _{2} \pm 0.001$ and $3.9 \log _{2} \pm 0.010$ fold for variant 15 and 23 respectively. Variant 14 did not show any expression for rpsU in 2 out of 3 replicates and a $C_{t}>30$ for the third replicate, indicating a severe downregulation in this variant as well. The variants with an intact rpsU gene and upstream region showed rpsU expression similar to the WT. 

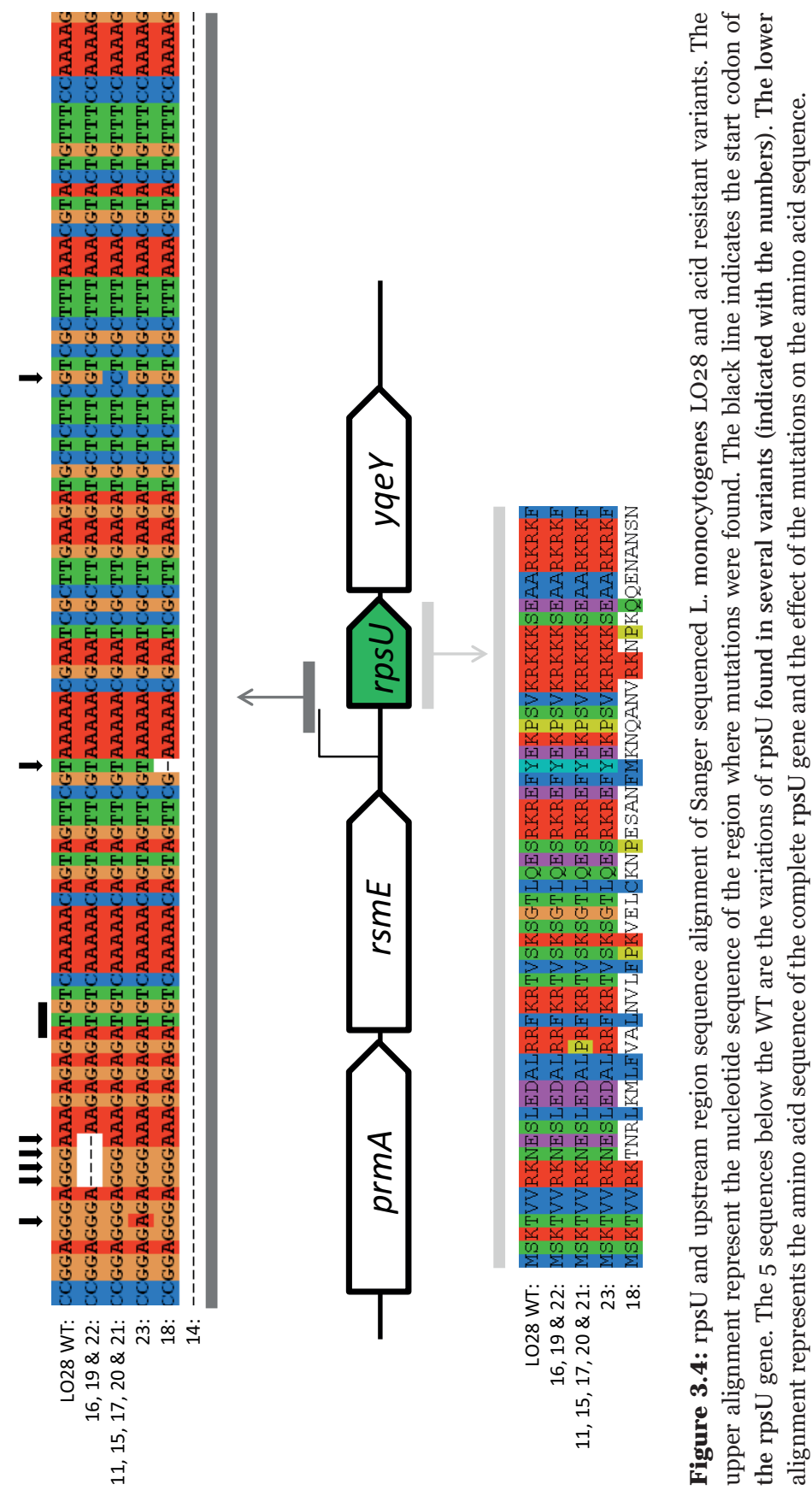


\section{Phenotypic clustering}

Hierarchical clustering was performed on the seven model variants and the WT (Figure 3.5) based on all the 14 measured phenotypic characteristics. The seven variants and WT were divided in 5 clusters. The first cluster included the WT and slightly resistant variant 3 (Cluster A). This variant only displayed slightly increased acid resistance, but for all the other phenotypes it showed similar behaviour to the WT. The second cluster contains variants 14, 15 and 23 (Cluster B). These variants were characterized by increased survival under acid, heat and BAC stress, increased $\mathrm{GAD}$ activity, reduced growth rate at $7^{\circ} \mathrm{C}$, normal motility, normal biofilm formation and normal hemolysis.

\section{A}

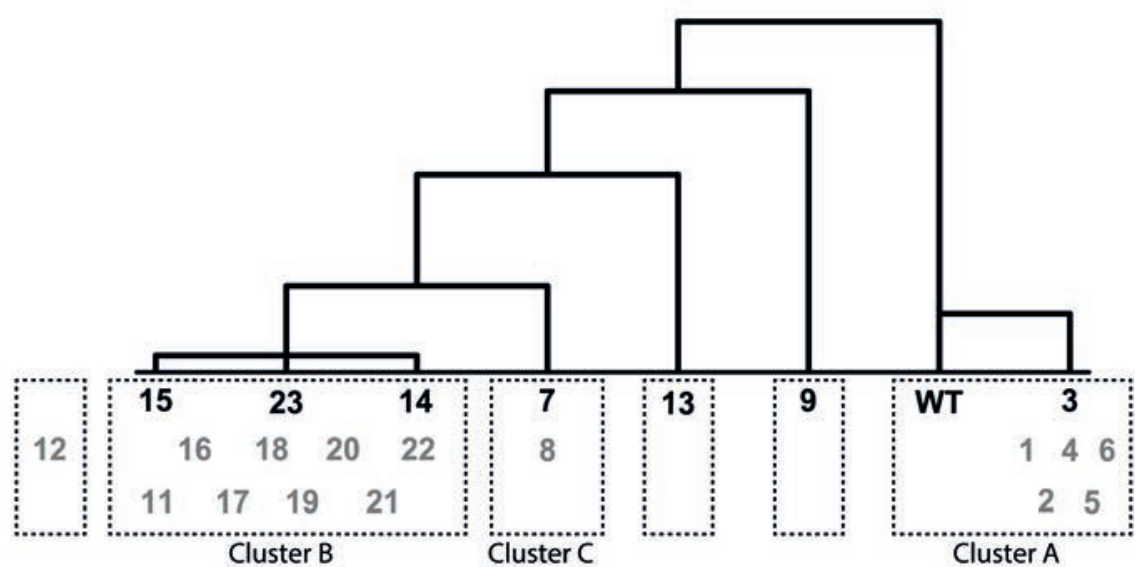

B

acid resistance growth $37^{\circ} \mathrm{C}$ growth $7^{\circ} \mathrm{C}$ phospholipase hemolysis motility
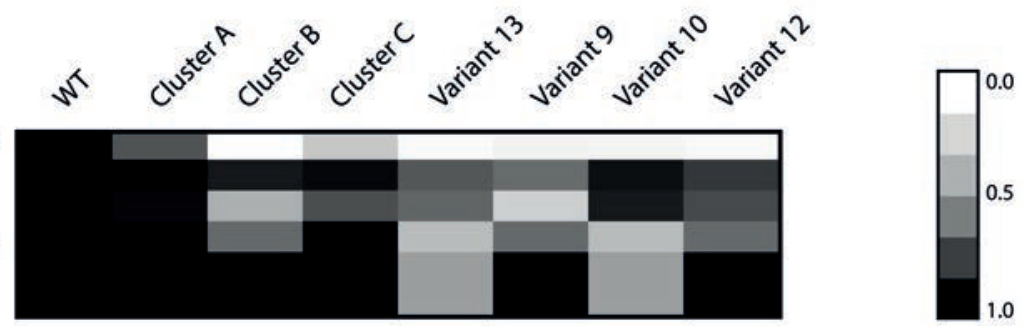

Figure 3.5: Hierarchical cluster analysis based on Euclidean distance of the phenotypes (A) and heat map of the 6 phenotypes for each cluster (B). The variants in black were the initial selection for cluster analysis and the grey variants were used to validate the resulting clusters. The heat map was made by taking the average of each phenotype within one cluster. Subsequently, the relative ratio of the cluster to the WT was calculated for each phenotype.

There were 3 variants which did not cluster with another variant. Variant 9 was characterized by reduced growth rate under all measured conditions, increased sensitivity to $\mathrm{H}_{2} \mathrm{O}_{2}$ but increased resistance to heat and acid. Variant 7 was very close to cluster $\mathrm{B}$, but with its more intermediate acid resistance and higher grouth 
rate, not part of this cluster. Variant 13 fell out of the larger cluster, mainly due to its reduced motility, hemolysis and phospholipase-C activity and the fact that it did not show reduced growth at $7^{\circ} \mathrm{C}$ compared to the $\mathrm{WT}$, which was characteristic for cluster B. In order to compare the remaining 16 variants to the seven model variants and to confirm that these seven are representative of the diversity within the population, the phenotypic clustering was extended with the remaining 16 variants. This was done based on the 6 phenotypic characteristics that were known for all 23 variants (acid resistance, growth characteristics, motility, hemolysis and phospholipase-C). All slightly acid resistant variants (1, 2, 4, 5 and 6) were grouped in cluster A. The largest cluster (B) consisted of the variants that showed highly increased acid resistance, combined with severely reduced growth rate at $7^{\circ} \mathrm{C}$ but unaffected growth rate at $37^{\circ} \mathrm{C}$. Eleven of the 23 variants fell within this cluster B. Interestingly, these are exactly the 11 variants in which a mutation in rpsU was identified. Variant 8 did not show significant differences with variant 7 on any of the 6 phenotypes, despite the fact that variant 7 was initially comprising its own group, due to its variable behaviour. These variants formed cluster C. Two variants could not be placed in any of the clusters, namely variants 10 and 12 , which mostly show the phenotypes of cluster $B$, apart from the fact that they do not show the dramatically reduced growth rate at $7^{\circ} \mathrm{C}$ which is typical for cluster B. On top of that, variant 12 showed a significantly lower growth rate at $37^{\circ} \mathrm{C}$ and variant 10 shows reduced motility, phospholipase and hemolysis (data not shown). Based on these phenotypes it can be concluded that 3 clusters and 4 individual variants were distinguished within the 23 acid resistant variants.

\section{Discussion}

During the transition from soil to human, L. monocytogenes encounters several adverse environmental conditions [1]. Genotypic diversity within the bacterial population may allow for elevated survival and growth in these different niches. The presence of several small, but highly resistant subpopulations increases the fitness of the population as a whole as it enables survival of several adverse conditions the population might encounter. Our current findings are an important step in unravelling the mechanisms of highly increased resistance of stress resistant variants. The phenotypic characterization indicated a wide diversity within the L. monocytogenes LO28 WT population and that different phenotypic characteristics are selected for by applying different types of stress. Based on acid resistance, the 23 variants could be divided in 3 groups; slightly increased acid resistance, variable acid resistance and highly increased acid resistance [9]. Although all variants were isolated after acid exposure ( $\mathrm{pH} 3.5$ for $90 \mathrm{~min}$ ), many of the variants were characterized by resistance towards other types of stress as well. The observed phenotypic diversity is in line with 
the variants isolated after HHP treatment, which also showed a large phenotypic diversity and multiple stress resistance [2]. Some of the phenotypic characteristics observed in the HHP resistant variants were observed in the acid resistant variants as well. Heat resistance and acid resistance were strongly correlated in both types of variants, as well as the impaired growth under a range of environmental conditions. However, also differences between HHP- and acid selected variants were observed. Comparison of thephenotypic clusters indicates that themajority of theHHP resistant variants cannot be classified in any of the phenotypic clusters of the acid resistant variants. The possibility of overlapping mechanisms of increased resistance in variants isolated after either HHP stress or acid stress cannot be completely excluded based on the phenotypic characterization alone, but it seems that the majority in both groups of variants is specific to the type of stress after which the variants have been isolated. This was also confirmed by the genotypic characterization. None of the acid resistant variants had a mutation in the ctsR gene or upstream region. On the other hand, all 11 members of cluster B were shown to have a mutation in or upstream of their rpsU gene, whereas this mutation was not found in the same region of any of the other 12 variants. The clear correlation of this mutation with one phenotypic cluster strongly suggests the mutations in this region to be related to the growth defects and increased stress resistance of this cluster of variants. The fact that these mutations alter the upstream region or affect the amino acid sequence of the gene itself, together with downregulation of rpsU in late-exponential phase cells of the rpsU variants, suggests a potential role of rpsU in stress resistance. The rpsU gene encodes the $30 \mathrm{~S}$ small subunit ribosomal protein S21. In L. monocytogenes rpsU is located between rsmE (a putative 16S rRNA methyltransferase) and yqeY (hypothetical protein). Interestingly, variant 14 had a large deletion, covering rpsU, yqeY and part of phoH but it clustered phenotypically with the other rpsU variants. Apparently, the loss of expression of yqeY and phoH encoded proteins did not have additional phenotypic effects in the tested conditions. There is not much known about the specific function of ribosomal protein S21 in L. monocytogenes or about its role in stress resistance in general, but in E. coli and Bacillus subtilis some more work has been done. A $\Delta$ rpsU mutant in B. subtilis showed unusual ribosome profiles, a reduced growth rate, and reduced motility [27]. Although motility was unaffected in the L. monocytogenes rpsU variants, the reduced growth rate was also observed. The fact that the ribosomal profiles were different in the B. subtilis mutants indicated that S21 is an important protein in correct functioning of the ribosomes [27] but the effect of knocking-out rpsU in B. subtilis on its stress resistance was not evaluated. Deletion of the genes encoding ribosomal proteins L31 and L25 in B. subtilis did result in phenotypes with increased stress resistance [28, 29]. Some recent proteome and transcriptome studies in L. monocytogenes found differential expression of ribosomal proteins upon stress 
exposure [30-33]. Also, a role in cold adaptation and cold stress response of L. monocytogenes has been suggested for specific ribosomal proteins [32-34]. Notably, in other microorganisms expression of S21 was suggested to be temperatureregulated. In the cyanobacterium Anabaena variabilis, the transcription of the rbpArpsU operon was upregulated at low temperature $\left(22^{\circ} \mathrm{C}\right)$ compared to high temperature $\left(38^{\circ} \mathrm{C}\right)[35]$ which was correlated with higher levels of protein $\mathrm{S} 21$ at low temperature [36]. In the soil bacterium Sinorhizobium meliloti rpsU is located in a cold shock operon with cspA. The authors suggest that increased synthesis of S21 might function to compensate for the reduced initiation of translation, caused by the low temperature [37]. Our data suggest that also in L. monocytogenes protein S21 plays a role in growth at low temperature, since all rpsU variants show a severely reduced growth rate in $\mathrm{BHI}$ at $7^{\circ} \mathrm{C}$ compared to the WT. This growth defect is still visible at $30^{\circ} \mathrm{C}[9]$ but restored at $37^{\circ} \mathrm{C}$. The link between rpsU and acid resistance or the effect of rpsU on the regulation of any of the stress response genes of $\mathrm{L}$. monocytogenes has not been studied to our knowledge. One possibility is that the stress resistance of the variants is a direct effect of the reduced growth rate, since it has been reported that reduced growth rate increases the stress resistance of $\mathrm{L}$. monocytogenes [38]. The inverse correlation between acid resistance of the variants and the growth rate under the conditions at which the cells are grown prior to acid exposure (BHI, $\left.30^{\circ} \mathrm{C}[9]\right)$ supports this hypothesis. The energy invested in increased stress resistance seems to be at the expense of the growth rate. Whereas in one environment the increased stress resistance might be a major advantage, the reduced growth rate might pose a disadvantage in other competitive environments [39]. A reduced growth rate was observed in the ctsR mutants [2] as well, most likely as a consequence of the constant activation of the chaperones under the regulation of ctsR which is a costly process for the cell. The reduced growth rate can therefore also be considered as a trade-off for the increased stress resistance. One clear phenotype that was observed in not only the three rpsU variants but also variants 7 and 13 is the increased glutamate decarboxylase activity. The glutamate decarboxylase system is one of the most important acid survival mechanisms in L. monocytogenes. Although the system, its function and its exact mechanism have been studied intensively over the last years, also many aspects are not clear yet. For L. monocytogenes $\mathrm{LO} 28$ it is known that the glutamate/ GABA antiporters are not active in BHI and that only the intracellular GAD system is responsible for the conversion of glutamate into GABA [15]. In general, increased levels of GABA have been correlated to increased acid resistance of several $\mathrm{L}$. monocytogenes strains. In five of the seven variants in which the $\mathrm{GAD}_{\mathrm{i}}$ activity was determined, an approximately 2-fold increase in activity compared to the WT was observed. This is in line with previous data reported by Karatzas et al. [12] who observed that the $2 \log _{10}$ higher survival upon acid exposure of the WT compared to the gad mutants corresponded with around 2-fold higher 
GAD activity. Although higher GABA, levels were observed in the variants, this was not reflected by an increased gene expression of the gad genes. One possibility is that earlier during growth these genes were transiently higher expressed in the selected acid resistant variants but showing no detectable differences in mRNA levels in stationary phase. Since the gad genes are regulated by sigB [40], expression levels of sigB were determined as well (data not shown) and were not found to be significantly different between WT and variants. Since the GAD activity under the measured conditions solely depends on the $\mathrm{GAD}_{\mathrm{i}}$ system, and therefore on intracellular pools of glutamate, it can be speculated that the increased GABA concentrations are caused by a higher concentration of intracellular glutamate in these five variants compared to the WT. Until recently, the L. monocytogenes ctsR gene appeared to be the main mutation hot spot in natural variants isolated after single stress exposure to heat and high pressure [8]. It was surprising that acid exposure did not lead to selection for ctsR variants, although according to van Boeijen et al. [2], the ctsR variants also show increased resistance to $\mathrm{pH} 2.5$ for $3 \mathrm{~min}$. Studying the inactivation kinetics of ctsR variant HHP6 at 3.5 showed that the ctsR variant is equally sensitive as the LO28 WT (data not shown). Considering the estimated fraction of ctsR mutants in the LO28 WT population of $6.5 \cdot 10^{-6}[8]$, there will be a probability of 1 in $\sim 100.000$ of isolating a ctsR mutant after exposure to $\mathrm{pH}$ 3.5. This could explain that no ctsR variants were isolated after $90 \mathrm{~min}$ at $\mathrm{pH} 3.5$ and strengthens the observation that different types of stress, as well as different levels of stress lead to the selection for different types of variants. The large variety of phenotypes that were isolated after randomly chosen durations of stress exposure highlights the large phenotypic diversity within the L. monocytogenes population. The combination of phenotypic characterization and whole genome sequencing proved to be a powerful tool in getting new insights in the population diversity of L. monocytogenes and possible mechanisms involved in the observed stress tolerance. However, the genetic background for the increased resistance of the other variants remains unclear. This could be due to insufficient read coverage of specific genomic regions or the presence of deletions or insertions larger than about 6 bp that were not taken into account in the SV analysis. To our knowledge, it has not been reported before that a mutation and subsequent down-regulation of one of the ribosomal proteins in L. monocytogenes can be advantageous for the bacterial cell under selected conditions by increasing its stress resistance. The tradeoff for this increased resistance seems to be the reduced growth ability, especially at low temperature. The impact of these mutations and the corresponding trade-offs on food safety should be studied in more detail. Also, further investigation on a molecular and transcriptome level will provide us with a better understanding of the role of ribosomal proteins in general stress resistance and their role in the generation of genotypic heterogeneity within bacterial populations. 


\section{References}

1. Freitag, N.E., G.C. Port, and M.D. Miner, Listeria monocytogenes - From saprophyte to intracellular pathogen. Nature Reviews Microbiology, 2009. 7(9): p. 623-628.

2. Van Boeijen, I.K.H., A.A.E. Chavaroche, W.B. Valderrama, R. Moezelaar, M.H. Zwietering, and T. Abee, Population diversity of Listeria monocytogenes LO28: Phenotypic and genotypic characterization of variants resistant to high hydrostatic pressure. Applied and Environmental Microbiology, 2010. 76(7): p. 2225-2233.

3. Van Boeijen, I.K.H., R. Moezelaar, T. Abee, and M.H. Zwietering, Inactivation kinetics of three Listeria monocytogenes strains under high hydrostatic pressure. J ournal of Food Protection, 2008. 71(10): p. 2007-2013.

4. Karatzas, K.A.G. and M.H.J. Bennik, Characterization of a Listeria monocytogenes Scott A isolate with high tolerance towards high hydrostatic pressure. Applied and Environmental Microbiology, 2002. 68(7): p. 3183-3189.

5. Hauben, K.J .A., D.H. Bartlett, C.C.F. Soontjens, K. Cornelis, E.Y. Wuytack, and C.W. Michiels, Escherichia coli mutants resistant to inactivation by high hydrostatic pressure. Applied and Environmental Microbiology, 1997. 63(3): p. 945-950.

6. Sagarzazu, N., G. Cebrián, R. Pagán, S. Condón, and P. Mañas, Emergence of pulsed electric fields resistance in Salmonella enterica serovar Typhimurium SL1344. International J ournal of Food Microbiology, 2013. 166(2): p. 219-225.

7. Karatzas, K.A.G., J.A. Wouters, C.G.M. Gahan, C. Hill, T. Abee, and M.H.J. Bennik, The CtsR regulator of Listeria monocytogenes contains a variant glycine repeat region that affects piezotolerance, stress resistance, motility and virulence. Molecular Microbiology, 2003. 49(5): p. 1227-1238.

8. Van Boeijen, I.K.H., C. Francke, R. Moezelaar, T. Abee, and M.H. Zwietering, Isolation of highly heat-resistant Listeria monocytogenes variants by use of a kinetic modeling-based sampling scheme. Applied and Environmental Microbiology, 2011. 77(8): p. 2617-2624.

9. Metselaar, K.I., H.M.W. Den Besten, T. Abee, R. Moezelaar, and M.H. Zwietering, Isolation and quantification of highly acid resistant variants of Listeria monocytogenes. International J ournal of Food Microbiology, 2013. 166(3): p. 508-514.

10. Cotter, P.D., K. O'Reilly, and C. Hill, Role of the glutamate decarboxylase acid resistance system in the survival of Listeria monocytogenes LO28 in low pH foods. J ournal of Food Protection, 2001. 64(9): p. 1362-1368.

11. Feehily, C. and K.A.G. Karatzas, Role of glutamate metabolism in bacterial responses towards acid and other stresses. J ournal of Applied Microbiology, 2013. 114(1): p. 11-24.

12. Karatzas, K.A.G., L. Suur, and C.P. O'Byrne, Characterization of the intracellular glutamate decarboxylase system: Analysis of its function, transcription, and role in the acid resistance of various strains of Listeria monocytogenes. Applied and Environmental Microbiology, 2012. 78(10): p. 35713579.

13. Biesta-Peters, E.G., M.W. Reij, H. Joosten, L.G.M. Gorris, and M.H. Zwietering, Comparison of two optical-density-based methods and a plate count method for estimation of growth parameters of Bacillus cereus. Applied and Environmental Microbiology, 2010. 76(5): p. 1399-1405.

14. Tsukatani, T., T. Higuchi, and K. Matsumoto, Enzyme-based microtiter plate assay for $\gamma$-aminobutyric acid: Application to the screening of $\gamma$-aminobutyric acid-producing lactic acid bacteria. Analytica Chimica Acta, 2005. 540(2): p. 293-297.

15. Karatzas, K.A.G., O. Brennan, S. Heavin, J. Morrissey, and C.P. O’Byrne, Intracellular accumulation of high levels of $\gamma$-aminobutyrate by Listeria monocytogenes 10403S in response to low $\mathrm{pH}$ : Uncoupling of $\gamma$-aminobutyrate synthesis from efflux in a chemically defined medium. Applied and Environmental Microbiology, 2010. 76(11): p. 3529-3537. 
16. O'Byrne, C.P., C. Feehily, R. Ham, and K.A.G. Karatzas, A modified rapid enzymatic microtiter plate assay for the quantification of intracellular $\gamma$-aminobutyric acid and succinate semialdehyde in bacterial cells. J ournal of Microbiological Methods, 2011. 84(1): p. 137-139.

17. Gründling, A., L.S. Burrack, H.G.A. Bouwer, and D.E. Higgins, Listeria monocytogenes regulates flagellar motility gene expression through MogR, a transcriptional repressor required for virulence. Proceedings of the National Academy of Sciences of the United States of America, 2004. 101(33): p. 12318-12323.

18. Rabinovich, L., N. Sigal, I. Borovok, R. Nir-Paz, and A.A. Herskovits, Prophage excision activates Listeria competence genes that promote phagosomal escape and virulence. Cell, 2012. 150(4): p. 792-802.

19. Vazquez-Boland, J.A., C. Kocks, S. Dramsi, H. Ohayon, C. Geoffroy, J. Mengaud, and P. Cossart, Nucleotide sequence of the lecithinase operon of Listeria monocytogenes and possible role of lecithinase in cell-to-cell spread. Infection and Immunity, 1992. 60(1): p. 219-230.

20. Coffey, A., F.M. Rombouts, and T. Abee, Influence of environmental parameters on phosphatidylcholine phospholipase C production in Listeria monocytogenes: A convenient method to differentiate L. monocytogenes from other Listeria species. Applied and Environmental Microbiology, 1996. 62(4): p. 1252-1256.

21. Pfaffl, M.W., A. Tichopad, C. Prgomet, and T.P. Neuvians, Determination of stable housekeeping genes, differentially regulated target genes and sample integrity: BestKeeper - Excelbased tool using pair-wise correlations. Biotechnology Letters, 2004. 26(6): p. 509-515.

22. Pfaffl, M.W., G.W. Horgan, and L. Dempfle, Relative expression software tool (REST) for group-wise comparison and statistical analysis of relative expression results in real-time PCR. Nucleic Acids Research, 2002. 30(9).

23. Boisvert, S., F. Laviolette, and J. Corbeil, Ray: simultaneous assembly of reads from a mix of high-throughput sequencing technologies. Journal of computational biology : A Journal Of Computational Molecular Cell Biology, 2010. 17(11): p. 1519-1533.

24. Aziz, R.K., D. Bartels, A.A. Best, M. DeJ ongh, T. Disz, R.A. Edwards, . . O O. Zagnitko, The RAST Server: rapid annotations using subsystems technology. BMC genomics, 2008. 9: p. 75.

25. Kent, W.J., BLAT--the BLAST-like alignment tool. Genome Research, 2002. 12(4): p. 656-64.

26. Cotter, P.D., S. Ryan, C.G.M. Gahan, and C. Hill, Presence of GadD1 glutamate decarboxylase in selected Listeria monocytogenes strains is associated with an ability to grow at low pH. Applied and Environmental Microbiology, 2005. 71(6): p. 2832-2839.

27. Akanuma, G., H. Nanamiya, Y. Natori, K. Yano, S. Suzuki, S. Omata, . . . F. Kawamura, Inactivation of ribosomal protein genes in Bacillus subtilis reveals importance of each ribosomal protein for cell proliferation and cell differentiation. J ournal of Bacteriology, 2012. 194(22): p. 62826291.

28. Höper, D., U. Völker, and M. Hecker, Comprehensive characterization of the contribution of individual SigB-dependent general stress genes to stress resistance of Bacillus subtilis. J ournal of Bacteriology, 2005. 187(8): p. 2810-2826.

29. Reder, A., D. Höper, U. Gerth, and M. Hecker, Contributions of individual $\sigma B$-dependent general stress genes to oxidative stress resistance of Bacillus subtilis. J ournal of Bacteriology, 2012. 194(14): p. 3601-3610.

30. Melo, J., P.W. Andrew, and M.L. Faleiro, Different assembly of acid and salt tolerance response in two dairy Listeria monocytogenes wild strains. Archives of Microbiology, 2013. 195(5): p. 339348.

31. Pleitner, A.M., V. Trinetta, M.T. Morgan, R.L. Linton, and H.F. Oliver, Transcriptional and phenotypic responses of Listeria monocytogenes to chlorine dioxide. Applied and Environmental Microbiology, 2014. 80(9): p. 2951-2963.

32. Durack, J., T. Ross, and J.P. Bowman, Characterisation of the transcriptomes of genetically diverse Listeria monocytogenes exposed to hyperosmotic and low temperature conditions reveal global stress-adaptation mechanisms. PLoS ONE, 2013. 8(9): p. e73603. 
33. Ivy, R.A., M. Wiedmann, and K.J . Boor, Listeria monocytogenes grown at $7^{\circ} \mathrm{C}$ shows reduced acid survival and an altered transcriptional response to acid shock compared to L. monocytogenes grown at $37^{\circ} \mathrm{C}$. Applied and Environmental Microbiology, 2012. 78(11): p. 3824-3836.

34. Bayles, D.O., M.H. Tunick, T.A. Foglia, and A.J . Miller, Cold shock and its effect on ribosomes and thermal tolerance in Listeria monocytogenes. Applied and Environmental Microbiology, 2000. 66(10): p. 4351-4355.

35. Sato, N., A cold-regulated cyanobacterial gene cluster encodes RNA-binding protein and ribosomal protein S21. Plant Molecular Biology, 1994. 24(5): p. 819-823.

36. Sato, N., T. Tachikawa, A. Wada, and A. Tanaka, Temperature-dependent regulation of the ribosomal small-subunit protein S21 in the cyanobacterium Anabaena variabilis M3. J ournal of Bacteriology, 1997. 179(22): p. 7063-7071.

37. O'Connell, K.P. and M.F. Thomashow, Transcriptional organization and regulation of a polycistronic cold shock operon in Sinorhizobium meliloti RM1021 encoding homologs of the Escherichia coli major cold shock gene cspA and ribosomal protein gene rpsU. Applied and Environmental Microbiology, 2000. 66(1): p. 392-400.

38. Patchett, R.A., Watson, N., Fernandez, P.S., Kroll, R.G., The effect of temperature and growth rate on the susceptibility of Listeria monocytogenes to environmental stress conditions. Letters in Applied Microbiology, 1996. 22: p. 121-124.

39. Phan, K. and T. Ferenci, A design-constraint trade-off underpins the diversity in ecologically important traits in species Escherichia coli. ISME J ournal, 2013. 7(10): p. 2034-2043.

40. Wemekamp-Kamphuis, H.H., J.A. Wouters, P.P.L.A. De Leeuw, T. Hain, T. Chakraborty, and T. Abee, Identification of sigma factor oB-controlled genes and their impact on acid stress, high hydrostatic pressure, and freeze survival in Listeria monocytogenes EGD-e. Applied and Environmental Microbiology, 2004. 70(6): p. 3457-3466. 



\title{
4
}

\section{Performance of stress resistant variants of Listeria monocytogenes in mixed species biofilms with Lactobacillus plantarum}

\author{
K.I. Metselaar, P. Saá Ibusquiza, A.R. Ortiz Camargo, \\ M. Krieg, M.H. Zwietering, H.M.W. den Besten and T. Abee
}

Published in: International Journal of Food Microbiology (2015) 213: p. 24-30. 


\section{Abstract}

Population diversity and the ability to adapt to changing environments allows Listeria monocytogenes to grow and survive under a wide range of environmental conditions. In this study, we aimed to evaluate the performance of a set of acid resistant L. monocytogenes variants in mixed-species biofilms with Lactobacillus plantarum as well as their benzalkonium chloride (BAC) resistance in these biofilms. L. monocytogenes $\mathrm{LO}_{28}$ wild type and acid resistant variants were capable of forming mixed biofilms with L. plantarum at $20^{\circ} \mathrm{C}$ and $30^{\circ} \mathrm{C}$ in BHI supplemented with manganese and glucose. Homolactic fermentation of glucose by L. plantarum created an acidic environment with $\mathrm{pH}$ values below the growth boundary of $\mathrm{L}$. monocytogenes. Some of the variants were able to withstand the low $\mathrm{pH}$ in the mixed biofilms for a longer time than the WT and there were clear differences in survival between the variants which could not be correlated to (lactic) acid resistance alone. Adaptation to mild $\mathrm{pH}$ of liquid cultures during growth to stationary phase increased the acid resistance of some variants to a greater extent than of others, indicating differences in adaptive behaviour between the variants. Two variants that showed a high level of acid adaptation when grown in liquid cultures, showed also better performance in mixed species biofilms. There were no clear differences in BAC resistance between the wild type and variants in mixed biofilms. It can be concluded that acid resistant variants of $\mathrm{L}$. monocytogenes show diversity in their adaptation to acidic conditions and their capacity to survive in mixed cultures and biofilms with L. plantarum. 


\section{Introduction}

Contamination of food products with the human pathogen Listeria monocytogenes can occur through detachment and transfer of cells from biofilms present in food production lines [1]. Biofilms are complex structures and several environmental conditions, like temperature and nutrient availability, can affect the composition of these biofilms [2]. L. monocytogenes can be found as part of mixed species biofilms, making the biofilm structure even more complex than those of single biofilms [1, 3]. The spoilage organism Lactobacillus plantarum favours similar niches as L. monocytogenes, mostly related to similar preferences in carbohydrate utilization [4]. L. monocytogenes has been recovered from fermented food products where high levels of L. plantarum were present $[5,6]$. Furthermore, these two species have been shown to be able to form mixed biofilms in a range of conditions [7]. During L. plantarum growth on glucose, homolacticfermentation resultsin high concentrations of lactic acid [8]. Van der Veen and Abee [7] showed that the $\mathrm{pH}$ in mixed species biofilms of L. monocytogenes with L. plantarum in a glucose-rich medium can drop as low as $\mathrm{pH}$ 3.4, which is below the growth boundary of L. monocytogenes [9]. Even though L. monocytogenes normally does not survive well in such acidic conditions, it has been demonstrated that heterogeneity exists within bacterial populations and variants of L. monocytogenes have been isolated which are highly acid resistant [10]. These variants, which are stable acid resistant fractions of the WT population, are phenotypically and genotypically diverse. It has been shown that a large fraction of these variants, besides being highly acid resistant, display a multiple stress-resistant phenotype, which allows them to survive under harsh environmental conditions. Some of the variants were capable of forming dense biofilms in BHI and showed also increased survival to the quaternary ammonium compound (QAC) benzalkonium chloride (BAC) in late-exponential phase liquid cultures [11]. BAC is a disinfectant which acts on the bacterial cell membrane, causing leakage of the cytoplasm [12]. Because $\mathrm{BAC}$ is active against a wide range of spoilage organisms and pathogens it is widely used in food industry [12]. In general, cells in biofilms are more resistant to BAC than planktonic cells and the composition of the biofilm is an important determinant in biofilm resistance to BAC treatments [13]. It was shown that mixed species biofilms are even more resistant to disinfection treatments than single species biofilms [7]. The exact mechanisms underlying this increased resistance of mixed species biofilms is not exactly understood but seems to be dependent on the combination of species, the environmental conditions under which the biofilm was formed and the disinfection treatment used [14, 15]. The diversity as encountered within L. monocytogenes populations allows small resistant fractions to survive and grow under a wide range of environmental conditions. When these resistant 
populations are present in food production lines, they may form or get incorporated into biofilms. Survival and BAC resistance of L. monocytogenes variants might be differently affected than the WT in an acidic biofilm with L. plantarum. It is commonly known that growth history and acid adaptation of L. monocytogenes can affect its subsequent biofilm formation and acid resistance, which may increase robustness of cells when dispersed [16-19]. Information on behaviour of stressresistant L. monocytogenes variants in biofilms helps to evaluate the potential role of the L. monocytogenes population diversity in surface attachment and the risk of persistence in food processing facilities. The aim of this study therefore was to evaluate the performance of L. monocytogenes LO28 WT and acid resistant variants in mixed species biofilms with L. plantarum WCFS1 and to determine their BAC resistance.

\section{Materials and methods}

\section{Bacterial strains and culturing conditions}

Lactobacillus plantarum WCFS1 wild type [8] (Laboratory of Food Microbiology, Wageningen University, the Netherlands), Listeria monocytogenes LO28 wild type (Wageningen UR Food \& Biobased Research, the Netherlands) and eight Listeria monocytogenes $\mathrm{LO} 28$ acid resistant variants [10] were used in this study. The stock cultures were kept in $15 \%(\mathrm{v} / \mathrm{v})$ glycerol (Fluka) at $-80^{\circ} \mathrm{C}$, and before the experiments cells from the stock were grown for two days at $30^{\circ} \mathrm{C}$ on brain heart infusion (BHI) agar (Becton Dickinson (BD)) or De Man, Rogosa and Sharpe (MRS) agar (Merck), for L. monocytogenes and L. plantarum respectively.

\section{Mixed biofilms of L. monocytogenes and L. plantarum}

Overnight (ON) cultures of L. monocytogenes and L. plantarum were made by inoculating a single colony in $10 \mathrm{ml}$ BHI (BD) supplemented with $0.005 \%(\mathrm{w} / \mathrm{v})$ manganese sulphate and $2 \%(\mathrm{w} / \mathrm{v})$ glucose (BHI-Mn-Glu; both from Merck) as described by Van der Veen and Abee [7] and grown at $30^{\circ} \mathrm{C}(18-22 \mathrm{~h}) .3 \mathrm{ml}$ fresh BHI-Mn-Glu was added to each well of a 12-well plate (Greiner Bio-One) and each well was inoculated with $30 \mu \mathrm{L}$. monocytogenes $\mathrm{ON}$ culture and $30 \mu \mathrm{L}$. plantarum ON culture, corresponding to an initial concentration of $\sim 7.5 \log _{10} \mathrm{cfu} / \mathrm{ml}$ for each species. One well with only medium was used as a negative control. The 12-well plates were incubated at $20^{\circ} \mathrm{C}$ for $12,24,36$ and $48 \mathrm{~h}$ and at $30^{\circ} \mathrm{C}$ for 12,18 and $24 \mathrm{~h}$. $20^{\circ} \mathrm{C}$ was included because it represents ambient temperature and because $30^{\circ} \mathrm{C}$ is commonly used in literature this temperature was included for comparison reasons. 
The time points were chosen based on the different acidification rates at the two temperatures. After incubation, the biofilm supernatant was pipetted from the wells. Biofilm supernatants were used for $\mathrm{pH}$ measurements and cells were enumerated as described below. The biofilm was washed three times with $3 \mathrm{ml}$ phosphate buffered saline (PBS) and after the third wash, the biofilm cells were resuspended in $1 \mathrm{ml}$ PBS by rigorous pipetting as described by Van der Veen and Abee [7]. Serial dilutions were made in PBS for the biofilm cells and the biofilm supernatant cells and the appropriate dilutions were spread plated on MRSA for L. plantarum enumeration and ALOA (bioMérieux) for L. monocytogenes enumeration. MRSA plates were incubated in anaerobic jars and all plates were incubated for two days at $30^{\circ} \mathrm{C}$. Experiments were performed in independent triplicates.

\section{Benzalkonium chloride (BAC) resistance of mixed biofilms}

Biofilms cultured for $24 \mathrm{~h}$ at $20^{\circ} \mathrm{C}$ and biofilms cultured for $12 \mathrm{~h}$ at $30^{\circ} \mathrm{C}$ were made as described above by inoculation of $1 \mathrm{ml}$ BHI-Mn-Glu with $10 \mu \mathrm{l}$ ON culture of both L. monocytogenes and L. plantarum in duplicate wells. These time points were chosen since the L. monocytogenes cell counts and $\mathrm{pH}$ in the biofilms were similar at these time-temperature combinations. Resistance to BAC of the intact biofilms, biofilm cells that were resuspended in PBS and biofilm supernatant cells was determined as described below. BAC (Acros Organics, Belgium) solutions were made by diluting a stock solution of $50 \mathrm{~g} / \mathrm{L}$ in water to the appropriate concentration (200 mg/L for biofilms, $40 \mathrm{mg} / \mathrm{L}$ for resuspended biofilm cells and $20 \mathrm{mg} / \mathrm{L}$ for biofilm supernatant cells). The different cell types have a different BAC susceptibility [20] and concentrations were chosen to achieve a significant reduction in culturable counts after $15 \mathrm{~min}$ BAC exposure however with final numbers reached well above the detection limit. To test the BAC resistance of the intact biofilms, the supernatant was removed and the biofilm was washed three times with $1 \mathrm{ml}$ PBS. $1 \mathrm{ml}$ BAC solution was added to one well and $1 \mathrm{ml}$ PBS was added to the other well as a control. After 15 minutes BAC or PBS exposure, $1 \mathrm{ml}$ Difco D/E Neutralizing agent (BD, Le Point de Claix, France) was added and the cells were resuspended by pipetting rigorously. To test the BAC resistance of the resuspended biofilm cells the procedure for washing was similar as for washing the intact biofilms, but after 2 times washing with PBS, the biofilm cells were resuspended in $1 \mathrm{ml}$ of PBS by rigorous pipetting, spun down (Hereaus, Biofuge Pico) at $4000 \cdot \mathrm{g}$ and resuspended in $1 \mathrm{ml}$ BAC (exposure) or PBS (control). After $15 \mathrm{~min}, 500 \mu \mathrm{l}$ was added to $500 \mu \mathrm{l} \mathrm{D} / \mathrm{E}$ neutralizing agent. For the BAC resistance of the biofilm supernatant cells, the supernatants were separated from the biofilms, centrifuged (Hereaus, Biofuge Pico) at $4000 \cdot \mathrm{g}$ and washed in PBS three times (similar as three times washing with PBS for the biofilms) and after the last washing step resuspended in $1 \mathrm{ml}$ BAC or PBS. After $15 \mathrm{~min}, 500 \mu \mathrm{l}$ of this 
cell suspension was added to $500 \mu \mathrm{l}$ / E neutralizing agent. From all the neutralized cell suspensions (biofilms, resuspended biofilm cells and biofilm supernatant cells) serial dilutions were made in PBS and $100 \mu$ of the appropriate dilutions were plated on ALOA and MRSA. Plates were incubated and enumerated as described above. Experiments were performed in independent triplicates.

\section{Lactic acid survival of $L$. monocytogenes}

A single colony was used to inoculate $10 \mathrm{ml}$ BHI broth (Oxoid, Basingstoke, Engeland). After ON growth (18-22 h) at $30^{\circ} \mathrm{C}$ (Innova 4335; New Brunswick Scientific) with aeration at $200 \mathrm{rpm}, 0.5 \%$ (v/v) inoculum was added to $50 \mathrm{ml}$ fresh BHI broth in an Erlenmeyer flask. Cells were grown in $\mathrm{BHI}$ at $20^{\circ} \mathrm{C}$ or $30^{\circ} \mathrm{C}$ with aeration until stationary phase. Stationary phase was defined as 3-6 hours after reaching $\mathrm{N}_{\max }$ $\left(\sim 9.5 \log _{10} \mathrm{cfu} / \mathrm{ml}, \mathrm{OD}_{600} \sim 2.0\right)$. Acid inactivation experiments were performed as described by Metselaar et al. [10], but BHI was adjusted to $\mathrm{pH} 3.4$ [7] with 85\% lactic acid syrup (Sigma-Aldrich, the Netherlands). Briefly, $10 \mathrm{ml}$ stationary phase culture was centrifuged $(5 \mathrm{~min}, 2880 \cdot \mathrm{g}$ ) and resuspended in $0.5 \mathrm{ml}$ peptone physiological saline (PPS). This cell suspension was added to $4.5 \mathrm{ml} \mathrm{BHI}$ with lactic acid, prewarmed at $30^{\circ} \mathrm{C}$. Samples were taken after $\mathrm{t}=0 \mathrm{~min}$ (directly after adding the cells to BHI with lactic acid) and $t=25 \mathrm{~min}\left(20^{\circ} \mathrm{C}\right)$ or $\mathrm{t}=90 \mathrm{~min}\left(30^{\circ} \mathrm{C}\right)$ of lactic acid exposure. Serial dilutions were made in BHI broth and $50 \mu \mathrm{l}$ was plated in duplicate using a spiral plater (Eddy J et, IUL Instruments). Plates were incubated at $30^{\circ} \mathrm{C}$ and enumerated after 4-6 days. Results were expressed as reduction in $\log _{10} \mathrm{cfu} / \mathrm{ml}$ by taking the difference in $\log _{10} \mathrm{cfu} / \mathrm{ml}$ at $\mathrm{t}_{0}$ and $\mathrm{t}_{25}\left(20^{\circ} \mathrm{C}\right)$ or $\mathrm{t}_{90}\left(30^{\circ} \mathrm{C}\right)$. Experiments were performed in independent triplicates.

\section{The effect of growth history on acid resistance of $L$. monocytogenes}

A single colony was used to inoculate $10 \mathrm{ml}$ BHI broth (Oxoid, Basingstoke, England). After $\mathrm{ON}$ growth at $30^{\circ} \mathrm{C}$ with shaking at $200 \mathrm{rpm}, 0.5 \%(\mathrm{v} / \mathrm{v})$ inoculum was added into fresh BHI broth. Cells were grown with shaking in either $\mathrm{BHI}$ at $20^{\circ} \mathrm{C}$, BHI set to $\mathrm{pH} 5.0$ by $10 \mathrm{M} \mathrm{HCl}$ at $20^{\circ} \mathrm{C}$ and $30^{\circ} \mathrm{C}$ or in BHI-Mn-Glu at $20^{\circ} \mathrm{C}$ and $30^{\circ} \mathrm{C}$ until stationary phase. Stationary phase was defined as 3-6 hours after reaching $\mathrm{N}_{\max }$ $\left(\sim 9.5 \log _{10} \mathrm{cfu} / \mathrm{ml}, \mathrm{OD}_{600} \sim 2.0\right)$. Acid inactivation experiments were performed as described above but using BHI set at $\mathrm{pH} 2.5$ by $10 \mathrm{M} \mathrm{HCl}$ at $37^{\circ} \mathrm{C}$. Samples were taken at $\mathrm{t}=0 \mathrm{~min}$ and $\mathrm{t}=\mathrm{x}$ min, where $\mathrm{t}_{\mathrm{x}}$ was chosen for each growth condition aiming for a 3-5 $\log _{10}$ reduction for the WT. Serial dilutions were made in BHI broth and $50 \mathrm{\mu l}$ was plated in duplicate using a spiral plater. Plates were incubated at $30^{\circ} \mathrm{C}$ and enumerated after 4-6 days. Results were expressed as reduction in $\log _{10} \mathrm{cfu} / \mathrm{ml}$ by taking the difference in $\log _{10} \mathrm{cfu} / \mathrm{ml}$ at $\mathrm{t}_{0}$ and $\mathrm{t}_{\mathrm{x}}$. Experiments were performed in independent triplicates. 


\section{Statistical analysis}

Differences between WT and variants were evaluated by a Student t-test using Microsoft Excel. Differences were considered significantly different when $\mathrm{p}<0.05$.

\section{Results and discussion}

Heterogeneity exists within L. monocytogenes populations and different environmental conditions can drive selection for different types of stress resistant variants which subsequently can grow and survive under a wide range of environmental conditions [21-23]. In this study, we aimed to evaluate L. monocytogenes variants biofilm formation and survival, with and without added BAC in mixed species biofilms with L. plantarum. Behaviour of the different variants in biofilms was compared to behaviour in liquid cultures in order to get more insight in parameters that affect behaviour of the different variants in a biofilm lifecycle.

\section{Performance of $L$. monocytogenes WT and variants in mixed biofilms with $L$. plantarum}

The set of L. monocytogenes variants used in this study were highly acid resistant compared to the WT, both in late-exponential and stationary phase [10]. It was therefore hypothesized that this set of variants might also survive better in acidic environments, like the combination of low $\mathrm{pH}$ and high lactic acid concentrations encountered in mixed cultures and biofilms with L. plantarum in BHI-Mn-Glu [7]. Firstly, single species biofilm formation of L. monocytogenes WT and variants was determined in BHI-Mn-Gluat $20^{\circ} \mathrm{Cand} 30^{\circ} \mathrm{C}$. All thevariantswerecapable of forming dense single species biofilms at both temperatures in $24 \mathrm{~h}$. There was no significant difference between WT and variants with respect to single biofilm formation and cell counts of $8.8 \pm 0.3$ and $9.2 \pm 0.3$ were determined at $20^{\circ} \mathrm{C}$ and $30^{\circ} \mathrm{C}$ respectively (data not shown). When L. monocytogenes and L. plantarum WCFS1 were cultured together in BHI-Mn-Glu, acidification was accelerated and the $\mathrm{pH}$ dropped to $\sim 3.7$ after $48 \mathrm{~h}$ at $20^{\circ} \mathrm{C}$ (Figure 4.1) and after $24 \mathrm{~h}$ at $30^{\circ} \mathrm{C}$ (Figure 4.2). In Figure 4.1 it can be seen that after $12 \mathrm{~h}$ at $20^{\circ} \mathrm{C}$ the $\mathrm{pH}$ is 5.3 and that the WT and all the variants have formed a biofilm with L. plantarum. After $24 \mathrm{~h}$ at $20^{\circ} \mathrm{C}$, the L. plantarum numbers were increased to more than $9 \log _{10} \mathrm{cfu} /$ well, with a concomitant $\mathrm{pH}$ drop to $\mathrm{pH} 4.1$.

Despite a $\mathrm{pH}$ below the growth boundary in planktonic conditions [10], the L. monocytogenes numbers were still comparable to the numbers at $12 \mathrm{~h}$ for the WT and all variants. However, after $36 \mathrm{~h}$, at $\mathrm{pH} 3.8$, the WT and variant 3 counts in the biofilm significantly reduced to $\sim 5 \log _{10} \mathrm{cfu} /$ well, while all the other variants were 

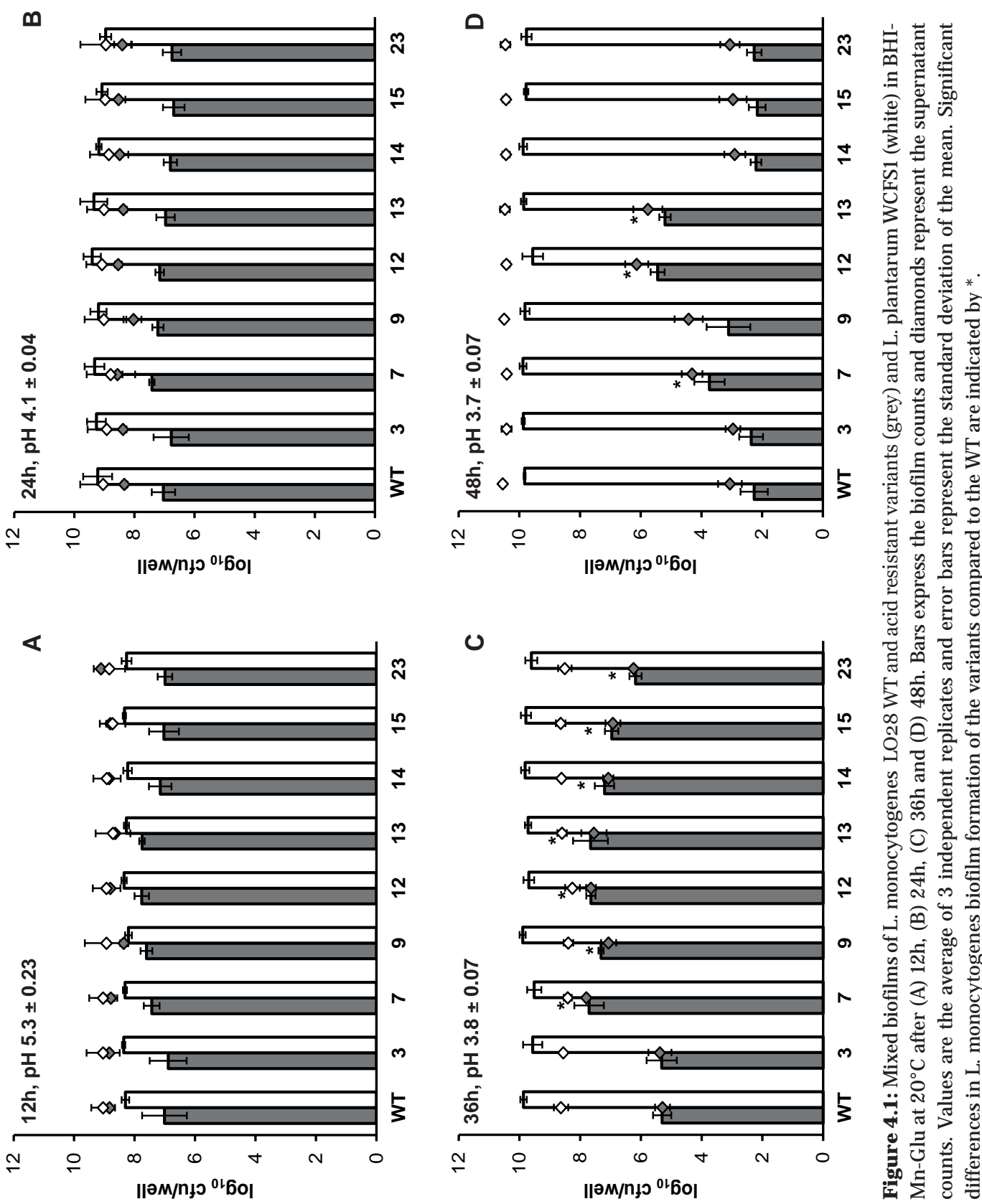
still present in high numbers ( $\sim 7 \log _{10}$ cfu/ well) comparable to 12 and $24 \mathrm{~h}$. After $48 \mathrm{~h}$, the $\mathrm{pH}$ was further reduced to 3.7 and a reduction in cell counts was noted for all L. monocytogenes variants. However, at this time point, variants 7, 12 and 13 showed significant higher culturable cell numbers than the WT and the other variants. At $30^{\circ} \mathrm{C}$ a similar trend was observed, but since the acidification was faster, the reduction in L. monocytogenes counts was also faster (Figure 4.2). After $12 \mathrm{~h}$, the $\mathrm{pH}$ was already 3.9, but the L. monocytogenes counts were still around $7 \log _{10}$ cfu/ well. After $18 \mathrm{~h}$, there is only $\sim 2 \log _{10}$ cfu/ well left for the WT and variant 3, whereas all the other variants still showed numbers around $6 \log _{10}$ cfu/ well, clearly indicating that these variants again show increased acid resistance in mixed species biofilms.

After 24h, the counts for the WT, variant 3, 7 and 9 have dropped below the detection limit ( $2 \log _{10} \mathrm{cfu} /$ well) while the other variants were still detectable. Interestingly, the supernatant counts were below the detection limit ( $1.6 \log _{10} \mathrm{cfu} /$ $\mathrm{ml}$ ) for all the variants at this time point, while the biofilm counts were still in the range of 2-4 $\log _{10}$ cfu/well. It was previously shown that even though the variants are more resistant to severe $\mathrm{pH}$ stress, they do not show better growth at mild $\mathrm{pH}$ stress ( $\mathrm{pH}$ 5.0) or a lower $\mathrm{pH}$ growth boundary [10].

It can therefore be concluded that the higher numbers after $24 \mathrm{~h}$ at $20^{\circ} \mathrm{C}$ and $12 \mathrm{~h}$ at $30^{\circ} \mathrm{C}$ are most likely not due to a lower growth boundary or better growth at low $\mathrm{pH}$ of the variants but to higher culturability after acid exposure. These data indicate that temperature plays a role in the performance of the different variants in biofilms. From these data it is not clear if the temperature has a direct effect on culturability of L. monocytogenes after acid stress exposure or if the differences in survival are related to the slower acidification rate of L. plantarum at lower temperature. Where at $20^{\circ} \mathrm{C}$ only variants 7,12 and 13 had an advantage over the WT in the mixed biofilm, at $30^{\circ} \mathrm{C}$ this was the case for variants $12,13,14,15$ and 23. It can therefore be concluded that the survival advantage of the variants is dependent on the environmental conditions. It can also be speculated that $\mathrm{L}$. monocytogenes enters a viable but not culturable (VNBC) state, as it has been shown previously that $\mathrm{L}$.monocytogenes can use this survival strategy under adverse environmental conditions [24-26]. L. monocytogenes VBNC cells have also been reported in biofilms for strain $\mathrm{NCTC} 13372$ at temperatures ranging from $4^{\circ} \mathrm{C}$ to $37^{\circ} \mathrm{C}$ [27]. However, in most studies on VBNC state of L. monocytogenes prolonged exposure to mild stress up to several days or weeks or starvation conditions (like biofilm formation in tap water) were used. Whether conditions in mixed species biofilms containing L. plantarum as secondary species induce VBNC state in L. monocytogenes remains to be elucidated. 

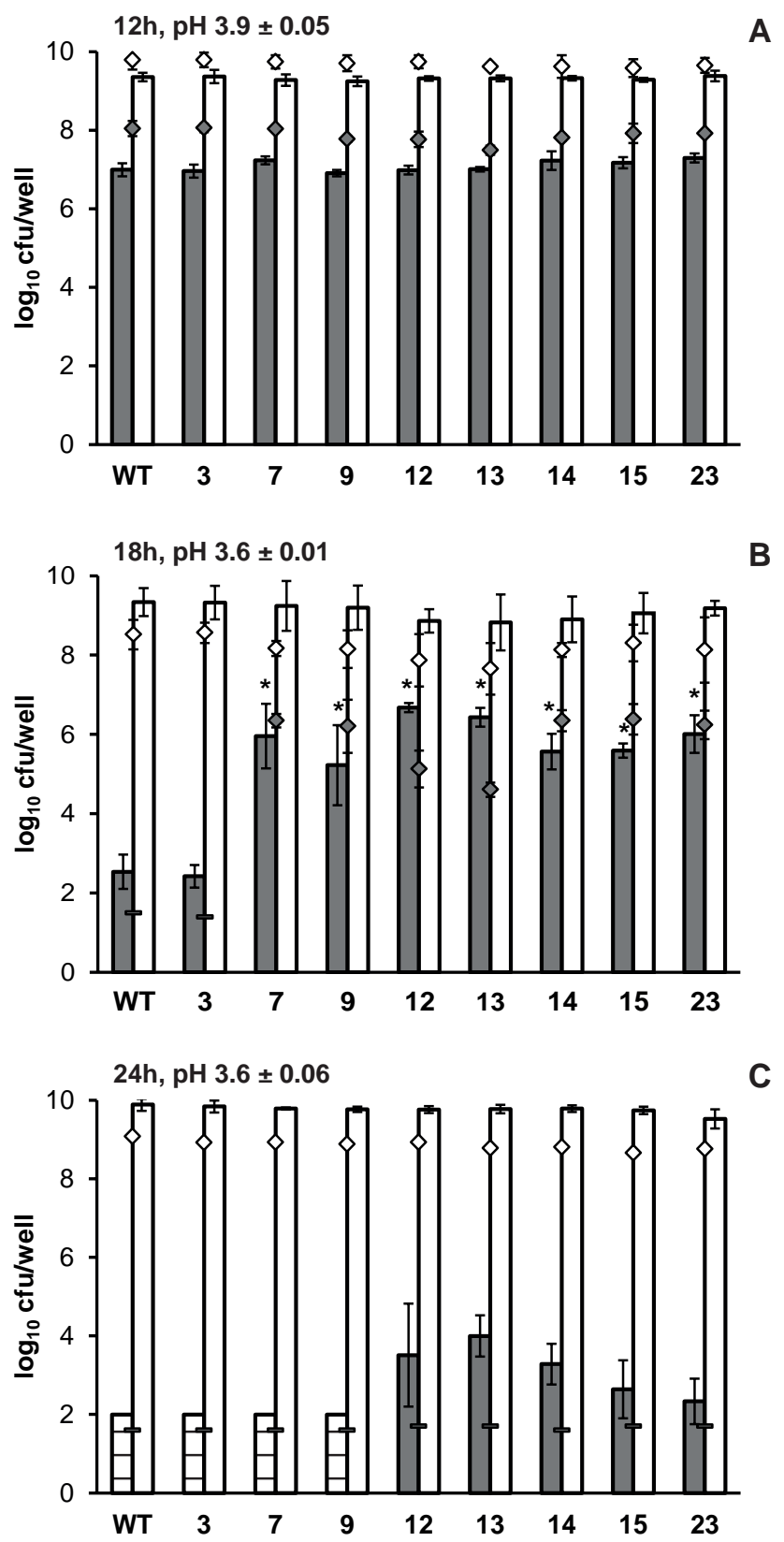

Figure 4.2: Mixed biofilms of L. monocytogenes LO28 WT and acid resistant variants (grey) and L. plantarum WCFS1 (white) in BHI-Mn-Glu at $30^{\circ} \mathrm{C}$ after (A) $12 \mathrm{~h}$, (B) $18 \mathrm{~h}$ and (C) $24 \mathrm{~h}$. Bars represent the biofilm counts and diamonds represent the supernatant counts. Significant differences in L. monocytogenes biofilm formation of the variants compared to the WT are indicated by*. Dashed bars indicate biofilm values below the detection limit $\left(2 \log _{10} \mathrm{cfu} / \mathrm{ml}\right.$ ) and dashes indicate values below the detection limit in supernatant counts $\left(1.6 \log _{10} \mathrm{cfu} / \mathrm{ml}\right)$. 


\section{Resistance to BAC of L. monocytogenes WT and variants in mixed biofilms with L. plantarum}

It was previously shown that planktonic late-exponential phase cells of variants 13, 14, 15 and 23 showed increased resistance towards BAC [23]. Therefore, the resistance of L. monocytogenes to BAC in the mixed biofilms was also evaluated and compared to that of resuspended biofilm cells and the biofilm supernatant cells. Cells from mixed species biofilms of $24 \mathrm{~h}$ at $20^{\circ} \mathrm{C}$ (Figure $4.3 \mathrm{~A}-\mathrm{C}$ ) and $12 \mathrm{~h}$ at $30^{\circ} \mathrm{C}$ (Figure 4.3D-F) were evaluated for BAC resistance, since the L. monocytogenes counts for the WT and all variants were comparable at these time points (Figure 4.1 and Figure 4.2). At both temperatures, the $\mathrm{pH}$ of the biofilms was around $\mathrm{pH} 4$, indicating that the cells were already stressed. In general, the resistance of the intact biofilms was significantly higher than the resistance of the resuspended biofilm cells, which was higher than the resistance of the supernatant cells, indicated by the higher BAC concentration needed to get a reduction in cell numbers (Figure 4.3). This is in line with previous studies and most likely related to the protective effect of the biofilm matrix, preventing BAC to penetrate into the deeper layers of the biofilm $[1,20,28]$. As can be seen in Figure 4.3, there was no obvious difference in BAC resistance between the WT and variants neither in biofilms, in resuspended biofilm cells or in supernatant cells.

In contrast to the previously observed BAC resistance of the late-exponential phase liquid culture cells of the variants compared to WT [23], this phenomenon was not observed in mixed biofilms with L. plantarum. One possible explanation for the different pattern of BAC resistance in biofilms compared to late-exponential phase planktonic cells might be the growth phase of the cells. The physiological state of cells embedded in a biofilm is different from planktonic exponential phase cells and this was shown to affect susceptibility to QACs $[1,13,20]$. It could be that this growth phase effect is stronger than the intrinsic BAC resistance of the variants. Another reason might be the effect of L. plantarum. Several authors have highlighted the importance of bacterial interactions within mixed-species biofilms of L. monocytogenes, not only with L. plantarum but also with Pseudomonas species for example $[14,29]$. The combination of species together with environmental factors has a large impact on the resistance of the cells within or detached from the biofilm when exposed to QAC and other disinfectants [7, 14]. Belessi et al. [28] found no difference in QAC survival between acid adapted and non-adapted L. monocytogenes cells, suggesting that acid adaptation is not a factor in BAC resistance of the biofilm cells or supernatant cells. It should be noted that, although BAC resistance of the variants is similar to WT, some variants have a higher probability of being present in significant numbers in biofilms with L. plantarum (Figure 4.1 and Figure 4.2) and therefore need more stringent disinfection. 

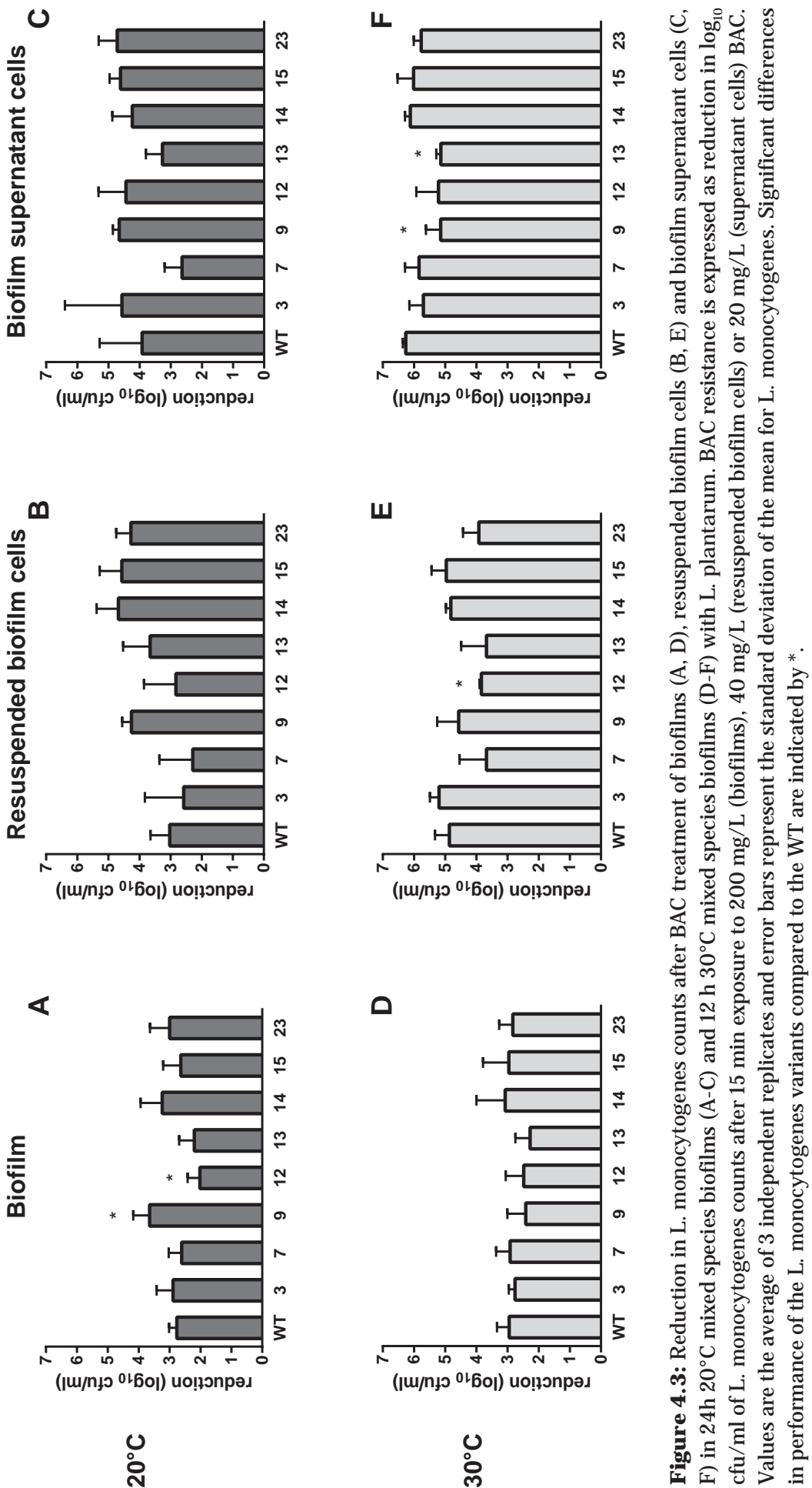

ำ
8 


\section{The effect of temperature and growth history on acid survival of L. monocytogenes variants}

To evaluate whether the increased survival of some variants in mixed species biofilms with L. plantarum can be attributed to acid resistance of the variants, the survival in biofilms was compared to the (lactic) acid shock survival at $20^{\circ} \mathrm{C}$ and $30^{\circ} \mathrm{C}$. In Figure 4.4A and Bit can be seen that at both temperatures, all the variants, except for variant 3 , showed increased resistance towards $\mathrm{BHI}$ set at $\mathrm{pH} 2.5$ by $\mathrm{HCl}$. The trend between $20^{\circ} \mathrm{C}$ and $30^{\circ} \mathrm{C}$ was comparable. The low pH that $\mathrm{L}$. monocytogenes encounters in the mixed species biofilms with L. plantarum is due to high concentration of lactic acid produced by glucose fermentation [30]. Therefore, the lactic acid resistance of cells grown at both temperatures was evaluated as well at low pH. In Figure S4.1 (supplementary data) it can be seen that most acid resistant variants also show increased resistance to low $\mathrm{pH}$ in the presence of lactic acid. When the acid resistance data of Figure 4.4A and B and S4.1 is compared to the survival in biofilms (Figure 4.1 and Figure 4.2) it can be concluded that survival in low $\mathrm{pH}$ biofilms cannot solely be predicted based on the acid resistance of a variant at a certain temperature. This raised the question if acid adaptation plays a role in the survival of the different variants in biofilms with L. plantarum. The gradual acidification of the environment by L. plantarum in the biofilms, which is temperature dependent, may allow for acid adaptation of L. monocytogenes. In several planktonic systems, the effect of acid adaptation on subsequent stress resistance has been studied extensively. Exponential-phase cells can induce an acid tolerance response (ATR) when shortly exposed to mild $\mathrm{pH}$ stress before lethal $\mathrm{pH}$ exposure. The ATR involves de novo protein synthesis and provides a protective effect towards lethal acid exposure [31, 32]. In general, at similar $\mathrm{pH}$ values $\mathrm{HCl}$ is less effective in inducing an ATR than lactic acid or acetic acid [31]. The WT and all variants were capable of inducing an ATR when grown until late-exponential phase at $30^{\circ} \mathrm{C}$, followed by 30 min exposure to $\mathrm{pH} 5.0$ set with $\mathrm{HCl}$ before lethal acid exposure of $\mathrm{pH} 2.5$ set with $\mathrm{HCl}$ (data not shown). Also stationary phase cells can acquire a level of protection to lethal $\mathrm{pH}$ stress by mild acid adaptation. L. monocytogenes grown under mild $\mathrm{pH}$ stress until stationary phase displays a higher level of acid resistance than cells grown under optimal $\mathrm{pH}[33,34]$. Also the medium and nutrient availability can affect subsequent stress resistance [35]. Figure 4.4 shows the effect of growth in different media on subsequent acid shock survival of planktonic L. monocytogenes WT and variants.

When grown in $\mathrm{BHI}$ set to $\mathrm{pH} 5.0$ by $\mathrm{HCl}$ at $30^{\circ} \mathrm{C}$ (Figure 4.4D) until stationary phase, especially variants 12 and 13 were more resistant than the WT. This might direct to a better acid adaptation behaviour of these three variants compared to the others. In BHI-Mn-Glu, the mild acid stress was also encountered, but the $\mathrm{pH}$ in the growth medium is gradually reduced to around $\mathrm{pH} 5.0$ by lactic acid production, allowing the microorganisms to slowly adapt to the mild $\mathrm{pH}$ stress. 


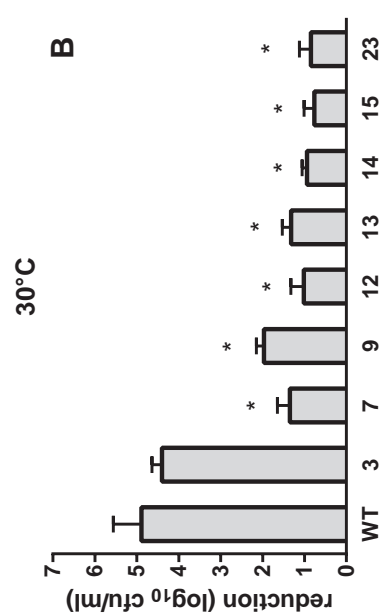

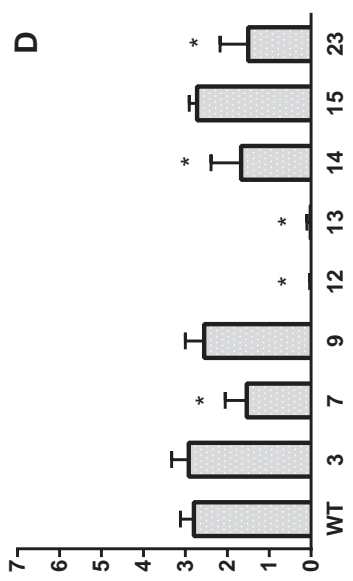

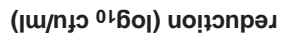
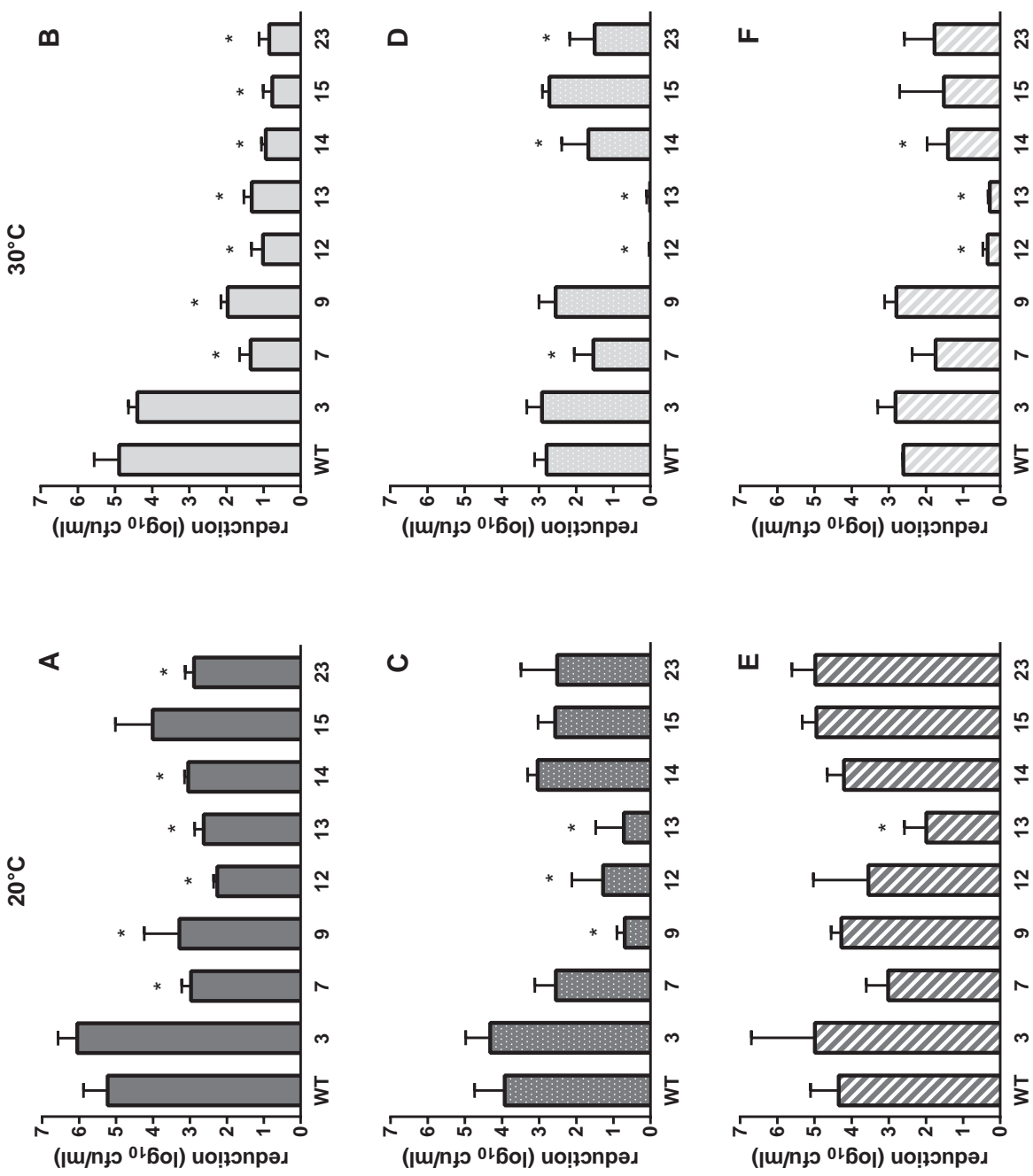

폼

포ํ 웁 
The overall pattern between cells grown in BHI at pH 5.0 and in BHI-Mn-Glu is very similar at $30^{\circ} \mathrm{C}$. However, when the WT and variants were grown at $\mathrm{pH} 5.0$ and in BHI-Mn-Glu at $20^{\circ} \mathrm{C}$, there is a clear difference between the two media. All the variants were more sensitive to $\mathrm{pH} 2.5$ when grown in BHI-Mn-Glu than when grown at $\mathrm{pH} 5.0$ at $20^{\circ} \mathrm{C}$. Interestingly, none of the variants, except for variant 13 , showed significantly higher culturability after acid shock than the WT when grown in BHI-Mn-Glu at $20^{\circ} \mathrm{C}$. Also when grown in $\mathrm{pH} 5.0$ at $20^{\circ} \mathrm{C}$ only variant 9,12 and 13 were significantly more acid resistant than the WT. At both temperatures, variants 12 and 13 showed higher culturability than the WT. These variants showed a similar acid resistance to the other highly resistant variants when grown in $\mathrm{BHI}$ at $30^{\circ} \mathrm{C}$ (both towards $\mathrm{HCl}$ and lactic acid), but when grown in either BHI-Mn-Glu or BHI set to $\mathrm{pH}$ 5.0, these two variants have an advantage over the other variants with respect to acid resistance. Interestingly, this advantage is also reflected in the higher culturability of these two variants in the biofilms.

\section{Population heterogeneity in biofilms and the potential role in persistence}

From the combination of the data from planktonic cells and that in mixed biofilms, it appears that especially variants 12 and 13 more efficiently adapt to mild acid stress than the other variants and that this protects them against acid shock due to the low $\mathrm{pH}$ in the mixed biofilm with L. plantarum. The other variants showed highly increased acid resistance when precultured under optimal conditions but this might be at the cost of their adaptation behaviour, resulting in comparable acid shock resistance as the WT when grown under mild stress conditions. These data underline the importance of diversity for the L. monocytogenes population. Whereas some variants (14, 15 and 23) are well suited to deal with rapid changes in the environment, other variants (12 and 13) seem to be better capable of adaptive behaviour. Especially the combination of all these different variants within the L. monocytogenes population should be considered when studying its behaviour and biofilm formation since in food processing environments a diverse mix of L. monocytogenes populations and strains may be present [36]. The increased survival capacities of the tested variants in this study, combined with their ability to form biofilms, may allow for persistence in food production lines. The acid resistant variants used in this study were isolated from short single acid exposure (90 min $\mathrm{pH}$ 3.5) but prolonged acid exposure has also been shown to lead to the isolation of acid resistant variants [31]. These variants seem to be phenotypically different from the ones used in our study, underlining the great diversity within the L. monocytogenes population. Notably, high hydrostatic pressure (HHP) resistant variants described by Van Boeijen et al. [21] also showed to be highly resistant to multiple types of stress, but many of the variants were not capable of forming dense 
biofilms. These variants might therefore have less potential of persistence in biofilms than the currently described acid resistant variants. Furthermore, acid adapted acid resistant variants might have a higher chance on stomach survival when they get detached from the biofilm and end up in food products. Overall, it can be concluded that heterogeneity in the L. monocytogenes population allows it to cope with several adverse conditions, not only when grown under optimal condition in planktonic state but also under less favorable culturing conditions and in biofilm growth mode. The higher stress resistance and adaptive behavior of some variants are potential risk factors in persistence. 


\section{References}

1. Møretrø, T. and S. Langsrud, Listeria monocytogenes: biofilm formation and persistence in foodprocessing environments. Biofilms, 2004. 1(02): p. 107-121.

2. Donlan, R.M. and J.W. Costerton, Biofilms: Survival mechanisms of clinically relevant microorganisms. Clinical Microbiology Reviews, 2002. 15(2): p. 167-193.

3. Carpentier, B. and D. Chassaing, Interactions in biofilms between Listeria monocytogenes and resident microorganisms from food industry premises. International J ournal of Food Microbiology, 2004. 97(2): p. 111-122.

4. Siezen, R., J . Boekhorst, L. Muscariello, D. Molenaar, B. Renckens, and M. Kleerebezem, Lactobacillus plantarum gene clusters encoding putative cell-surface protein complexes for carbohydrate utilization are conserved in specific gram-positive bacteria. BMC Genomics, 2006. 7: p. article number 126.

5. Caggia, C., C.L. Randazzo, M.D.I. Salvo, F. Romeo, and P. Giudici, Occurrence of Listeria monocytogenes in green table olives. J ournal of Food Protection, 2004. 67(10): p. 2189-2194.

6. Campanini, M., I. Pedrazzoni, S. Barbuti, and P. Baldini, Behaviour of Listeria monocytogenes during the maturation of naturally and artificially contaminated salami: effect of lactic-acid bacteria starter cultures. International J ournal of Food Microbiology, 1993. 20(3): p. 169-175.

7. Van der Veen, S. and T. Abee, Mixed species biofilms of Listeria monocytogenes and Lactobacillus plantarum show enhanced resistance to benzalkonium chloride and peracetic acid. International J ournal of Food Microbiology, 2011. 144(3): p. 421-431.

8. Kleerebezem, M., J . Boekhorst, R. van Kranenburg, D. Molenaar, O.P. Kuipers, R. Leer, ... R.J. Siezen, Complete genome sequence of Lactobacillus plantarum WCFS1. Proceedings of the National Academy of Sciences, 2003. 100(4): p. 1990-1995.

9. Van der Veen, S., R. Moezelaar, T. Abee, and M.H.J. Wells-Bennik, The growth limits of a large number of Listeria monocytogenes strains at combinations of stresses show serotype- and nichespecific traits. J ournal of Applied Microbiology, 2008. 105(5): p. 1246-1258.

10. Metselaar, K.I., H.M.W. Den Besten, T. Abee, R. Moezelaar, and M.H. Zwietering, Isolation and quantification of highly acid resistant variants of Listeria monocytogenes. International J ournal of Food Microbiology, 2013. 166(3): p. 508-514.

11. Metselaar, K.I., H.M.W. den Besten, J. Boekhorst, S.A.F.T. van Hijum, M.H. Zwietering, and T. Abee, Diversity of acid stress resistant variants of Listeria monocytogenes and the potential role of ribosomal protein S21 encoded by rpsU. Frontiers in Microbiology, 2015. 6:422.

12. McDonnell, G. and A.D. Russell, Antiseptics and disinfectants: Activity, action, and resistance. Clinical Microbiology Reviews, 1999. 12(1): p. 147-179.

13. Chavant, P., B. Gaillard-Martinie, and M. Hébraud, Antimicrobial effects of sanitizers against planktonic and sessile Listeria monocytogenes cells according to the growth phase. FEMSMicrobiology Letters, 2004. 236(2): p. 241-248.

14. Giaouris, E., N. Chorianopoulos, A. Doulgeraki, and G.J. Nychas, Co-culture with Listeria monocytogenes within a dual-species biofilm community strongly increases resistance of Pseudomonas putida to benzalkonium chloride. PLoS ONE, 2013. 8(10): p. e77276.

15. Pan, Y., F. BreidtJ r, and S. Kathariou, Resistance of Listeria monocytogenes biofilms to sanitizing agents in a simulated food processing environment. Applied and Environmental Microbiology, 2006. 72(12): p. 7711-7717.

16. Skandamis, P.N., A.S. Gounadaki, I. Geornaras, and J.N. Sofos, Adaptive acid tolerance response of Listeria monocytogenes strains under planktonic and immobilized growth conditions. International J ournal of Food Microbiology, 2012. 159(2): p. 160-166.

17. Giaouris, E., N. Chorianopoulos, and G.-J. Nychas, Acquired acid adaptation of Listeria monocytogenes during its planktonic growth enhances subsequent survival of its sessile population to disinfection with natural organic compounds. Food Research International, 2014. 64(0): p. 896-900.

18. Chorianopoulos, N., E. Giaouris, I. Grigoraki, P. Skandamis, and G.-J. Nychas, Effect of acid tolerance response (ATR) on attachment of Listeria monocytogenes Scott A to stainless steel under extended exposure to acid or/and salt stress and resistance of sessile cells to subsequent strong acid challenge. International J ournal of Food Microbiology, 2011. 145(2- 3): p. 400-406. 
19. Giaouris, E., N. Chorianopoulos, and G.-J. Nychas, Impact of acid adaptation on attachment of Listeria monocytogenes to stainless steel during long-term incubation under low or moderate temperature conditions and on subsequent recalcitrance of attached cells to lethal acid treatments. International J ournal of Food Microbiology, 2014. 171(0): p. 1-7.

20. Stopforth, J.D., J. Samelis, J.N. Sofos, P.A. Kendall, and G.C. Smith, Biofilm formation by acid-adapted and nonadapted Listeria monocytogenes in fresh beef decontamination washings and its subsequent inactivation with sanitizers. J ournal of Food Protection, 2002. 65(11): p. 1717-1727.

21. Van Boeijen, I.K.H., A.A.E. Chavaroche, W.B. Valderrama, R. Moezelaar, M.H. Zwietering, and T. Abee, Population diversity of Listeria monocytogenes LO28: Phenotypic and genotypic characterization of variants resistant to high hydrostatic pressure. Applied and Environmental Microbiology, 2010. 76(7): p. 2225-2233.

22. Van Boeijen, I.K.H., C. Francke, R. Moezelaar, T. Abee, and M.H. Zwietering, Isolation of highly heat-resistant Listeria monocytogenes variants by use of a kinetic modeling-based sampling scheme. Applied and Environmental Microbiology, 2011. 77(8): p. 2617-2624.

23. Metselaar, K.I., H.M.W. Den Besten, J . Boekhorst, S.A.F.T. Van Hijum, M.H. Zwietering, and T. Abee, Diversity of acid stress resistant variants of Listeria monocytogenes and the potential role of rpsU, encoding ribosomal protein S21. Frontiers in Microbiology, 2015. 6:422

24. Besnard, V., M. Federighi, E. Declerq, F. J ugiau, and J.M. Cappelier, Environmental and physico-chemical factors induce VBNC state in Listeria monocytogenes. Veterinary Research, 2002. 33(4): p. 359-370.

25. Li, L., N. Mendis, H. Trigui, J.D. Oliver, and S.P. Faucher, The importance of the viable but non-culturable state in human bacterial pathogens. Frontiers in Microbiology, 2014. 5(J UN): p. article number 258.

26. Lindbäck, T., M.E. Rottenberg, S.M. Roche, and L.M. Rørvik, The ability to enter into an avirulent viable but non-culturable (VBNC) form is widespread among Listeria monocytogenes isolates from salmon, patients and environment. Veterinary Research, 2010. 41(1).

27. Gião, M.S. and C.W. Keevil, Listeria monocytogenes can form biofilms in tap water and enter into the viable but non-cultivable state. Microbial ecology, 2014. 67(3): p. 603-611.

28. Belessi, C.E.A., A.S. Gounadaki, A.N. Psomas, and P.N. Skandamis, Efficiency of different sanitation methods on Listeria monocytogenes biofilms formed under various environmental conditions. International J ournal of Food Microbiology, 2011. 145(SUPPL. 1): p. S46-S52.

29. Saá Ibusquiza, P., J J .R. Herrera, D. Vázquez-Sánchez, and M.L. Cabo, Adherence kinetics, resistance to benzalkonium chloride and microscopic analysis of mixed biofilms formed by Listeria monocytogenes and Pseudomonas putida. Food Control, 2012. 25(1): p. 202-210.

30. Saá Ibusquiza, P., M. Nierop Groot, A. Debán-Valles, T. Abee, and H.M.W. den Besten, Impact of growth conditions and role of sigB on Listeria monocytogenes fitness in single and mixed biofilms cultured with Lactobacillus plantarum. Food Research International, 2015. 71(0): p. 140-145.

31. O'Driscoll, B., C.G.M. Gahan, and C. Hill, Adaptive acid tolerance response in Listeria monocytogenes: Isolation of an acid-tolerant mutant which demonstrates increased virulence. Applied and Environmental Microbiology, 1996. 62(5): p. 1693-1698.

32. Davis, M.J., P.J. Coote, and C.P. O'Byrne, Acid tolerance in Listeria monocytogenes: The adaptive acid tolerance response (ATR) and growth-phase-dependent acid resistance. Microbiology, 1996. 142(10): p. 2975-2982.

33. Kroll, R.G. and R.A. Patchett, Induced acid tolerance in Listeria monocytogenes. Letters in Applied Microbiology, 1992. 14(5): p. 224-227.

34. Samelis, J., J.S. Ikeda, and J.N. Sofos, Evaluation of the pH-dependent, stationary-phase acid tolerance in Listeria monocytogenes and Salmonella Typhimurium DT104 induced by culturing in media with 1\% glucose: a comparative study with Escherichia coli O157:H7. Journal of Applied Microbiology, 2003. 95(3): p. 563-575.

35. Herbert, K.C. and S.J . Foster, Starvation survival in Listeria monocytogenes: Characterization of the response and the role of known and novel components. Microbiology, 2001. 147(8): p. 2275-2284.

36. Ferreira, V., M. Wiedmann, P. Teixeira, and M.J. Stasiewicz, Listeria monocytogenes persistence in food-associated environments: Epidemiology, strain characteristics, and implications for public health. J ournal of Food Protection, 2014. 77(1): p. 150-170. 


\section{Supplemental data}
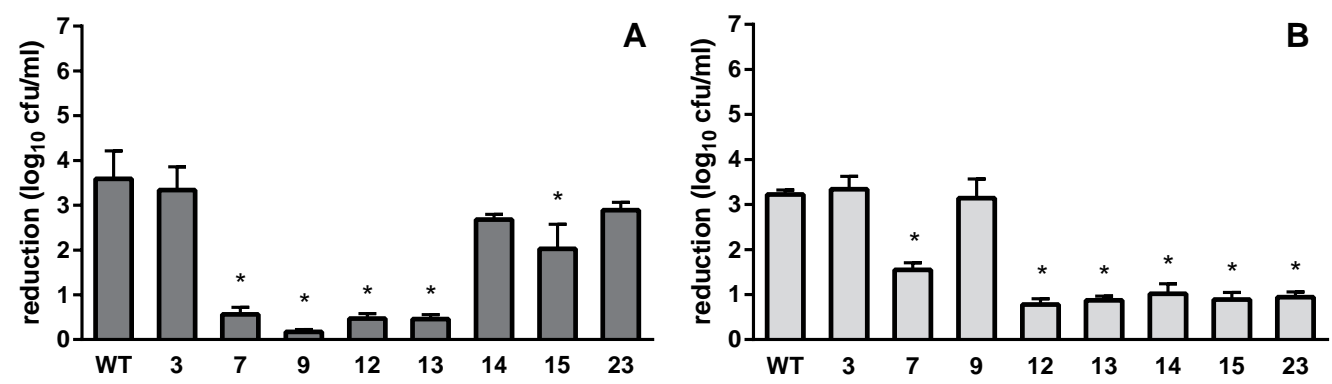

Figure S4.1: Reduction of stationary-phase cells of L. monocytogenes LO28 WT and acid resistant variants after exposure to $\mathrm{BHI}$ set at $\mathrm{pH} 3.4$ by $85 \%$ lactic acid syrup at $30^{\circ} \mathrm{C}$. Cells were grown to stationary phase in $\mathrm{BHI}$ at $20^{\circ} \mathrm{C}(\mathrm{A})$ or $30^{\circ} \mathrm{C}(\mathrm{B})$. Resistance is expressed as the reduction in viable counts after $25 \mathrm{~min}$ (A) and $90 \mathrm{~min}$ (B) of lactic acid exposure. Values are the average of 3 independent replicates. Error bars represent the standard deviation of the mean and statistically significant differences from the WT $(\mathrm{p}<0.05)$ are indicated by*. 


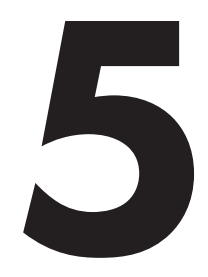

\section{Modeling and validation of ecological behaviour of Listeria monocytogenes wild type and stress resistant variants}

K.I. Metselaar, T. Abee, M.H. Zwietering and H.M.W. den Besten

Submitted for publication 


\section{Abstract}

Listeria monocytogenes exhibits a heterogeneous response upon stress exposure which can be partially attributed to the presence of stable stress resistant variants. A heterogeneous population allows for growth and survival under a wide range of environmental conditions with specific growth fitness and stress robustness parameters considered a trade-off. A variant may have an advantage in one condition, while this might be a disadvantage in other conditions. This study aims to evaluate the impact of the presence of stress resistant variants and their corresponding tradeoffs on population composition under different environmental conditions. A set of robustness and fitness parameters of WT and variants was obtained and used to model their growth behaviour under combined mild stress conditions and to model their kinetics in a simulated food chain. Predictions of performance were validated in single and mixed cultures by plate counts and by qPCR by which L. monocytogenes WT and an rpsU deletion variant could be distinguished with specific primers. Growth predictions for the $\mathrm{WT}$ and the rpsU deletion variant matced the experimental data generally well. Validation of performance in the simulated food chain confirmed the trend of higher fitness and lower robustness for the WT compared to the rpsU variant. This study provides insights into the conditions which can select for stress resistant variants in industrial settings and discusses their potential persistence in food processing environments. 


\section{Introduction}

Diversity exists within bacterial populations which can for example be observed by tailing of inactivation curves upon stress exposure. This tail can be caused by either phenotypic or genotypic heterogeneity. Phenotypic heterogeneity refers to transiently increased resistance with a physiological or epigenetic background. Reasons for phenotypic heterogeneity can be amongst others the presence of persisters [1], bistability caused by noise in stochastic gene expression [2-4] or epigenetic phenotype switching [5]. Genotypic heterogeneity refers to the presence of stable stress resistant variants, with an inheritable stress resistant phenotype caused by genomic alterations [6]. These stable stress resistant variants have been repeatedly isolated from the foodborne pathogen Listeria monocytogenes upon exposure to different types of stress, e.g. heat, high hydrostatic pressure (HHP) and low $\mathrm{pH}$ [7-11]. L. monocytogenes stress resistant variants have been shown to comprise a wide range of phenotypic features that are different from the main WT population. Apart from resistance towards the selection stress, numerous variants showed a multiple stress resistant phenotype [12, 13]. It was shown that different types of stress lead to selection for different types of variants. Although many phenotypic features are overlapping, the genetic basis for the increased resistance was shown to be partially selection-stress dependent [12]. A large fraction of the heat and HHP selected variants had a mutation in the class III heat shock repressor gene ctsR $[11,13]$, while this mutation was not found in the acid stress selected variants. In theacid stress selected variants a mutation in rpsU, encoding ribosomal protein S21, appeared to be responsible for the stress resistant phenotype in a large fraction of these variants [12]. An interesting observation in all these variants is that increased stress resistance seems to be at the cost of another feature. The growth rate for many of the variants was lower than for the WT, although in somecases it was only impaired under specific conditions. Generally, the most resistant variants showed a significant growth defect and a clear correlation between acid resistance and growth rate was established [9]. The increased stress resistance of the variants can be considered a trade-off; a variant may have an advantage under a certain condition, while having a disadvantage under another condition. The concept of stress resistance as a trade-off for growth has been well described in literature [14-16]. Specialization on survival often comes at the cost of slower multiplication. The underlying mechanism can be found in resource allocation; energy expenditure has to be divided over vegetative growth and general stress response [14]. This resource allocation is affected by the environmental conditions, butalso mutations have been shown to cause a shift in the 
distribution of resources between reproduction and maintenance [15]. The ctsR and rpsU mutations in the stress resistant $\mathrm{L}$. monocytogenes variants seem to result in a shift away from rapid reproduction and towards increased stress response. During food processing and storage, bacterial cells encounter different environments which can affect the behavior of the cells. Heat resistance has been shown to be affected by environmental conditions like growth temperature, medium composition and growth stage [17]. Growth at mild $\mathrm{pH}$ stress can induce increased resistance towards lethal acid stress but also towards other stress responses like thermal stress [18]. It was also shown that the different variants have a different degree of mild stress adaptive behavior, affecting their stress resistance [19]. The differences in behavior and resource allocation between WT and variants might have consequences for their growth and survival under processing conditions. Information on whether these stress resistant variants are a potential threat for food industry or if the disadvantages, combined with theirlow prevalencein thepopulation, counterbalance the increased resistance is currently lacking. It would be of relevance to evaluate the impact of the presence of stress resistance variants and their corresponding trade-offs on population composition. Therefore, this study aimed to quantify the growth and inactivation kinetics of L. monocytogenes acid resistant variants and WT under different environmental conditions and to use obtained quantitative data to predict the behavior along a simulated model food chain. It was hypothesized that the WT has an advantage during growth but that the variants have an advantage during inactivation steps. A modelling approach was used to predict the behavior of WT and variants under different combinations of mild and severe stress conditions, and the genetic background of one rpsU variant was used to validate predictions in mixtures in which WT and an rpsU deletion variant were distinguished by specific primers. This study highlights the potential impact of the environmental conditions on population dynamics of L. monocytogenes WT and its acid resistant rpsU variant in sequential niches.

\section{Materials and Methods}

\section{Bacterial strains and culturing conditions}

Listeria monocytogenes LO28 wild type (Wageningen UR Food \& Biobased Research, the Netherlands) and eight acid resistant variants isolated from the tail of this WT after acid exposure [9] were used in this study. The selected variants were 3, 7, 9, 12, $13,14,15 \& 23$, based on their genotypic and phenotypic characteristics representing different clusters $[12,19]$. The stock cultures were kept in $15 \%$ (v/v) glycerol (Fluka) at $-80^{\circ} \mathrm{C}$, and before the experiments cells from stock were grown for 2 days at $30^{\circ} \mathrm{C}$ 
on brain heart infusion (BHI; BD) agar plates. A single colony was used to inoculate $20 \mathrm{ml} \mathrm{BHI} \mathrm{broth} \mathrm{(Oxoid)} \mathrm{in} \mathrm{an} \mathrm{Erlenmeyer} \mathrm{flask.} \mathrm{After} \mathrm{overnight} \mathrm{(ON)} \mathrm{growth} \mathrm{(18-22}$ h) at $30^{\circ} \mathrm{C}$ (Innova 4335; New Brunswick Scientific, Edison, NJ) with shaking at 160 $\mathrm{rpm}, 0.5 \%(\mathrm{v} / \mathrm{v})$ inoculum was added to fresh $\mathrm{BHI}$ broth. Cells were grown in BHI at $30^{\circ} \mathrm{C}$ until late-exponential growth phase $\left(\mathrm{OD}_{600}=0.4-0.5\right.$ reached after $\left.4-5 \mathrm{~h}\right)$ or stationary phase (18-22 h of growth, $\mathrm{OD}_{600} \sim 2.0$ ). Bacteria were spiral plated (Eddy J et, IUL Instruments) or spread plated on BHI agar. For inactivation experiments, plates were incubated for $4-6$ days at $30^{\circ} \mathrm{C}$ to allow recovery of the cells. For all other experiments, plates were incubated for 2 days at $30^{\circ} \mathrm{C}$.

\section{Determination of the maximum specific growth rate}

The maximum specific growth rate $\left(\mu_{\max }\right)$ was determined under various mild stress conditions, namely at different $\mathrm{pH}$ conditions, water activities $\left(\mathrm{a}_{\mathrm{w}}\right)$ and temperatures (T). BHI broth (Oxoid; $\mathrm{a}_{\mathrm{w}} 0.997 ; \mathrm{pH} 7.3$ ) was used as a plain medium for all growth experiments with $30^{\circ} \mathrm{C}$ as the reference temperature. The effect of mild $\mathrm{pH}$ was determined by adjusting the $\mathrm{pH}$ of sterile $\mathrm{BHI}$ by $10 \mathrm{M} \mathrm{HCl}$ to $\mathrm{pH}$ 6.0, 5.0 and 4.7, followed by filter sterilization $(0.22 \mu \mathrm{m}$ filter). The water activity was lowered by adding $\mathrm{NaCl}$ (Merck) to $\mathrm{BHI}$ broth, resulting in $\mathrm{a}_{\mathrm{w}}$ values of $0.971,0.957,0.946$ and 0.940. Values for $\mu_{\max }$ at $7^{\circ} \mathrm{C}$ and $37^{\circ} \mathrm{C}$ were available from our previous study [9] and were extended in the current study at $20^{\circ} \mathrm{C}$ and $30^{\circ} \mathrm{C}$ to determine the effect of temperature on $\mu_{\max }$. The $\mu_{\max }$ for the WT and each variant was determined by the 2-fold dilution (2FD) method as described by Biesta-Peters et al. [20]. Briefly, $\mathrm{ON}$ cultures were used for this experiment and diluted in BHI broth to an initial concentration of $\sim 5 \cdot 10^{5} \mathrm{cfu} / \mathrm{ml}$ which was confirmed by plating on BHI agar plates. From this culture, five sequential 2-fold dilutions in BHI broth were made in duplicate in a 100 well honeycomb plate and the final volume in each well was $200 \mu$ l. The plate was incubated in the Bioscreen C (Oy Growth Curves AB Ltd). The Bioscreen $\mathrm{C}$ was set at the appropriate temperature with medium constant shaking and the $\mathrm{OD}_{600}$ was measured every 10 or 30 min depending on the conditions. This was done up to 2 weeks, or until all wells reached an $\mathrm{OD}_{600}$ of at least 0.2 (time to detection (TTD)). The $\mu_{\max }$ was determined for the WT and each variant for each condition by taking the negative reciprocal of the slope between TTD and $\ln \left(\mathrm{N}_{0}\right)$. For more stressful conditions in which an $\mathrm{OD}_{600}$ of 0.2 was barely reached, a TTD of 0.12 was used since 0.12 was in the linear part of the $\mathrm{OD}_{600}$ growth curve for all conditions and variants. Wells not showing an increase in $\mathrm{OD}_{600}$ during the time of the experiments were plated on BHI plates to confirm no growth. Experiments were repeated on different days using fresh cultures to obtain biologically independent duplicates or triplicates. 


\section{Estimating cardinal growth parameters}

Secondary growth models to estimate cardinal growth parameters were chosen based on the selection described by Aryani et al. [21]. The square root model [22] was used to describe the $\mu_{\max }$ as a function of temperature (T):

$$
\sqrt{\mu_{\max }}=b\left(T-T_{\min }\right)
$$

In which $\mathrm{b}$ is the slope parameter and $\mathrm{T}_{\min }\left({ }^{\circ} \mathrm{C}\right)$ is the temperature at which no growth is observed anymore. The $\mu_{\max }$ as a function of $\mathrm{pH}$ was described by the reparameterized model of Aryani et al. [21]:

$$
\mu_{\text {max }}=\mu_{\text {opt }}\left[1-2^{\frac{\left(p H-p H_{\min }\right)}{\left(p H_{\min }-p H_{1 / 2}\right)}}\right]
$$

In which $\mu_{\mathrm{opt}}\left(\mathrm{h}^{-1}\right)$ is the $\mu_{\max }$ at optimum $\mathrm{pH}, \mathrm{pH}_{\min }$ is the $\mathrm{pH}$ at which no growth is observed anymore and $\mathrm{pH}_{1 / 2}$ is the $\mathrm{pH}$ at which the $\mu_{\max }$ is half of the $\mu_{\mathrm{opt}}$. The $\mu_{\max }$ as a function of $\mathrm{a}_{\mathrm{w}}$ was described by the model of Luong et al. [23]:

$$
\mu_{\max }=\mu_{\text {opt }}\left[1-\left(\frac{1-a_{w}}{1-a_{w \min }}\right)^{d}\right]
$$

In which $\mu_{\text {opt }}\left(\mathrm{h}^{-1}\right)$ is the $\mu_{\max }$ at optimum $\mathrm{a}_{\mathrm{w}^{\prime}} \mathrm{a}_{\mathrm{w}, \min }$ is the $\mathrm{a}_{\mathrm{w}}$ at which no growth is observed anymore and $d$ is the shape parameter. The models were fitted to all independent replicate $\mu_{\max }$ data together using TableCurve 2D v5.01. For the $a_{w}$ model, a lack-of-fit test [24] was performed, in case 1 was in the confidence interval of $\mathrm{d}$, in order to get a more simple, linear model. The lack-of-fit test evaluates if the shape parameter can be removed by comparing the residual sum of squares (RSS) of the full model (with d) to the RSS of the reduced model (without d).

\section{Growth modeling}

Cardinal growth parameters obtained were used as input in the gamma model [25] to predict the growth of the WT and variants under combined mild stress conditions. The gamma model without inclusion of an interaction factor was used and therefore only a multiplicative effect of the combined mild stress conditions was assumed [26]. Predictions of $\mu_{\max }$ were made by:

$$
\mu_{\max }=\mu_{r e f} \cdot \gamma(T) \cdot \gamma(p H) \cdot \gamma\left(a_{w}\right)
$$

In which $\mu_{\text {ref }}\left(\mathrm{h}^{-1}\right)$ is the average of the estimated $\mu_{\max }$ in $\mathrm{BHI}$ at $30^{\circ} \mathrm{C}, \mathrm{pH} 7.3$ and $\mathrm{a}_{\mathrm{w}}$ 0.997 according to equation 1-3 and $\gamma(\mathrm{T}), \gamma(\mathrm{pH})$ and $\gamma\left(\mathrm{a}_{\mathrm{w}}\right)$ are the gamma factors for each individual condition $(\mathrm{x})$, which is determined by: 


$$
\gamma(x)=\frac{\mu_{\max }(x)}{\mu_{\text {ref }}}
$$

In which $\mu_{\max }(\mathrm{x})$ is calculated by equations 6-8 [21]:

$$
\begin{aligned}
& \mu_{\text {max }}(T)=\mu_{\text {ref }} \frac{\left(T-T_{\text {min }}\right)^{2}}{\left(T_{\text {ref }}-T_{\text {min }}\right)^{2}} \\
& \mu_{\text {max }}(p H)=\mu_{\text {ref }} \frac{\left(1-2^{\frac{\left(p H-p H_{\min }\right)}{\left(p H_{\text {min }}-p H_{1 / 2}\right)}}\right)}{\left(1-2^{\frac{\left(p H_{\text {ref }}-p H_{\min }\right)}{\left(p H_{\text {min }}\right)}}\right)} \\
& \mu_{\text {max }}\left(a_{w}\right)=\mu_{\text {ref }} \frac{\left(1-\left(\frac{1-a_{w}}{1-a_{w \min }}\right)^{d}\right)}{\left(1-\left(\frac{1-a_{w r e f}}{1-a_{w \min }}\right)^{d}\right)}
\end{aligned}
$$

The $\mu_{\max }$ values for combined stress conditions resulting from equation 4 were used as input into the widely used three-phase linear model [27] to predict the growth of the WT and variants under the defined conditions. The lag time $(\lambda)$ was assumed to be reciprocally proportional to $\mu_{\max }[24,28]$. The product $\mu_{\max } \cdot \lambda$ generally ranges between 0 and 4 [24] and therefore these values were considered to predict the growth intervals. Average $\mathrm{N}_{\max }$ was set at $9.5 \log _{10} \mathrm{cfu} / \mathrm{ml}$, based on counts of fully grown (ON) cultures. For the growth intervals, a range from 9.25 to $9.75 \log _{10} \mathrm{cfu} /$ $\mathrm{ml}$ was assumed.

\section{Heat inactivation}

Heat inactivation experiments were performed for the WT and rpsU variant 14 as described in [12]. This variant was chosen as a representative rpsU variant, based on the genetic background that allowed for validation as described below. Briefly, 400 $\mathrm{ll}$ late-exponential or stationary phase culture was transferred into $40 \mathrm{ml}$ preheated BHI broth in a $250 \mathrm{ml}$ Erlenmeyer flask, in a shaking (16o rpm) water bath (Julabo SW 23, J ulabo) at 55, 60 or $62^{\circ} \mathrm{C}$. At appropriate time intervals, a $1 \mathrm{ml}$ sample was taken and decimally diluted in PPS, or transferred into a sterile cup which was placed on ice in case the undiluted sample was to be plated. A separate Erlenmeyer 
containing $\mathrm{BHI}$ broth at room temperature was used for the $\mathrm{t}=0$ measurement. 50 $\mu \mathrm{l}$ of appropriate dilutions were spiral plated in duplicate on BHI agar or, in case of the undiluted samples, $1 \mathrm{ml}$ was spread plated (divided over 3 plates) resulting in a detection limit of $1 \mathrm{cfu} / \mathrm{ml}$. Subsequently, the resulting inactivation curves were described with any of the following models to determine the D-value. In the case of non-linear inactivation and the presence of a shoulder and tail, the reparameterized model of Geeraerd et al. [9, 29] (equation 9) was fitted to the data:

$$
\begin{aligned}
& \log _{10}\left(N_{t}\right)=\log _{10}\left(N_{0}\right)+\log _{10}\left(\left(1-10^{\log _{10}(f)}\right) \cdot \exp \left(-k_{\text {sens }} \cdot t\right) \cdot\right. \\
& \frac{\exp \left(k_{\text {sens }} \cdot S_{l}\right)}{1+\left(\exp \left(k_{\text {sens }} \cdot S_{l}\right)-1\right) \cdot \exp \left(-k_{\text {sens }} \cdot t\right)}+10^{\log _{10}(f)} \cdot \exp \left(-k_{\text {res }} \cdot t\right) \cdot \\
& \left.\left(\frac{\exp \left(k_{\text {sens }} \cdot S_{l}\right)}{1+\left(\exp \left(k_{\text {sens }} \cdot S_{l}\right)-1\right) \cdot \exp \left(-k_{\text {sens }} \cdot t\right)}\right)^{\frac{k_{\text {ess }}}{k_{\text {sens }}}}\right)
\end{aligned}
$$

In which $\mathrm{N}_{\mathrm{t}}$ is the number of cells at time (in min), $\mathrm{N}_{0}$ is the number of cells at $t=0$, $\mathrm{k}_{\text {sens }}$ and $\mathrm{k}_{\mathrm{res}}$ are the specific inactivation rates of the sensitive population and the resistant fraction, respectively $\left(\mathrm{min}^{-1}\right)$. $\mathrm{S}_{1}$ is the shoulder length (in $\mathrm{min}$ ) and $\mathrm{f}$ is the stress resistant fraction.

When the shoulder length $\left(\mathrm{S}_{1}\right)$ was not significant, equation 10 was fitted to the data:

$$
\begin{aligned}
& \log _{10}\left(N_{t}\right)=\log _{10}\left(N_{0}\right)+\log _{10}\left(\left(1-10^{\log _{10}(f)}\right) \cdot \exp \left(-k_{\text {sens }} \cdot t\right)+10^{\log _{10}(f)} \cdot \exp \right. \\
& \left.\exp \left(-k_{\text {res }} \cdot t\right)\right)
\end{aligned}
$$

When also the tail was not significant, the model was further reduced to a linear inactivation model:

$$
\log _{10}\left(N_{t}\right)=\log _{10}\left(N_{0}\right)+\exp (-k \cdot t)
$$

The D-value of the sensitive and resistant fractions for equation 9-11 was calculated by equation 12 :

$$
D=\frac{\ln (10)}{k}
$$

In the case that no tailing was present, a linear model with shoulder and the reparameterized Weibull model (equation 13) [9] was fitted to the data. The Weibull model was preferred as this model also considers the shoulder in D-value 
determination, whereas the linear model with shoulder does not.

$$
\log _{10}\left(N_{t}\right)=\log _{10}\left(N_{0}\right)-\Delta \cdot\left(\frac{t}{t_{\Delta D}}\right)^{\beta}
$$

in which $\Delta$ is the number of decimal reductions; $\mathrm{t}_{\Delta \mathrm{D}}$, the time needed to reduce the initial number of microorganism with $\Delta$ decimals (min); and $\beta$, a fitting parameter that defines the shape of the curve. $\Delta$ was set at 5 , since the measured data covered a range of at least $5 \log _{10}$ reductions, and the other parameters were estimated. All models were fitted to the data using TableCurve 2D v5.01. An F-test was performed to test if the fitting performance of the model was statistically accepted. The f value was calculated using the following equation [30]:

$$
f=\frac{M S E_{\text {model }}}{M S E_{\text {data }}}
$$

In which $\mathrm{MSE}_{\text {model }}$ is the mean square error of the model and $\mathrm{MSE}_{\text {data }}$ is the mean square error of the data for replicate values. The resulting f value was tested against a critical $\mathrm{F}$ table value with a 95\% confidence level. For the inactivation curves showing tailing and therefore described by equation 9 or 10, the reparameterized Weibull model (Equation 13) was also fitted to the data without taking the tail into consideration in order to be able to compare the WT and variant 14 at different temperatures and growth phases. The $\mathrm{D}$-values were calculated by dividing the $t_{5 \mathrm{D}}$ by 5 . From these D-values, the z-value was calculated for WT and variant 14 in lateexponential and stationary phase.

\section{Acid inactivation}

Acid inactivation experiments were performed as described in [9] for lateexponential and stationary phase cultures. Briefly, $10 \mathrm{ml}$ (stationary phase) or 50 $\mathrm{ml}$ (late-exponential phase cultures were centrifuged, resuspended in $1 \mathrm{ml}$ PPS and this cell suspension was added to $9 \mathrm{ml} \mathrm{BHI}$ set to $\mathrm{pH} 2.5$ by $10 \mathrm{M} \mathrm{HCl}$, prewarmed at $37^{\circ} \mathrm{C}$. The tube was placed in a shaking waterbath $(160 \mathrm{rpm})$ at $37^{\circ} \mathrm{C}$ and samples were taken at appropriate time intervals, directly diluted in BHI broth and spiral plated on BHI agar. Inactivation kinetics were described as was done for the heat inactivation data described above by Equations 9-12.

\section{DNA extraction and RT-PCR quantification}

DNA of the WT and variant 14, or mixtures of them was extracted from cell cultures by the Qiagen DNeasy Blood \&Tissue Kit, using thepre-treatment protocol for Grampositive bacteria with some modifications. Lysozyme and proteinase $\mathrm{K}$ incubations 
were done for $60 \mathrm{~min}$ and washing steps were repeated twice. Elution was done in $50 \mathrm{\mu l} 10 \mathrm{mM}$ Tris- $\mathrm{HCl} \mathrm{pH} 8.3$ and the eluate was diluted 10 times in nuclease-free water. DNA was stored at $4^{\circ} \mathrm{C}$ for up to 5 days until PCR quantification. Primers were designed in the rpsU region to specifically amplify only the WT or only the variant DNA. The WT specific primers (fw: 5'-CGCGCTTTCTGGATTCTTGC-3' rv: 5'-ACGAATCGCTTGAAGATGCTC-3') were designed within the rpsU gene, since variant 14 is lacking this gene completely. For variant 14 specific primers (fw: 5'-CGATGCCCGATGATTAAAA-3' rv 5'-GCGTCAACTGCCATAACAAC-3') were designed in such a way that the fw primer was bridging the 1300bp deletion of this variant and therefore this primer could only bind to variant 14 [12]. The primers were validated and confirmed to be specific. Quantification was done using a qPCR machine (Bio-Rad CFX96), at an annealing temperature of $60^{\circ} \mathrm{C}$ using Power SYBRgreen mastermix (Applied Biosystems). $\mathrm{C}_{\mathrm{t}}$ values were determined with automatic baseline settings. Standard curves were obtained from a series of decimally diluted DNA to determine PCR efficiency of the primers, and from DNA extractions from a series of decimally diluted $\mathrm{ON}$ cultures. The highest concentration was plated and a standard curve, correlating $\log _{10} \mathrm{cfu} / \mathrm{ml}$ to $\mathrm{c}_{\mathrm{t}}$ values was obtained in this way. This standard curve was used to quantify the number of viable cells in mixtures of WT and variant 14. The dynamic range of quantification was determined to be $\sim 4.5-9.5 \log _{10} \mathrm{cfu} / \mathrm{ml}$.

\section{Growth validations}

Growth predictions were validated for the WT and variant 14 in BHI for 3 conditions: $7^{\circ} \mathrm{C}, \mathrm{pH}$ 6.6, $\mathrm{a}_{\mathrm{w}} 0.997$ mimicking milk (BHI-M), $7^{\circ} \mathrm{C}, \mathrm{pH} 6.0, \mathrm{a}_{\mathrm{w}} 0.965$ mimicking ham (BHI-H) and $37^{\circ} \mathrm{C}, \mathrm{pH} 5.2, \mathrm{a}_{\mathrm{w}} 0.997$ providing conditions with similar expected growth for WT and variant 14 (BHI-S). Milk and ham conditions were taken from Aryani et al. [21]. BHI was prepared and the $\mathrm{a}_{\mathrm{w}}$ was set by adding $\mathrm{NaCl}$ before autoclaving. The $\mathrm{pH}$ was set after autoclaving and the medium was filter sterilized using a $0.22 \mu \mathrm{m}$ filter. Validations were done in Erlenmeyer flasks in a shaking incubator (200 rpm) set at the correct temperature. ON cultures (cultured in BHI at $30^{\circ} \mathrm{C}$ ) were diluted in the corresponding medium and inoculated in the Erlenmeyer flasks with a starting concentration of $\sim 4 \log _{10} \mathrm{cfu} / \mathrm{ml}$ as this was just below the detection limit of the PCR quantification method. This was done for the WT and variant 14 in single cultures and for WT and variant 14 mixed in equal amounts. For BHI-M and BHI-H, samples were taken once per day and for BHI-S this was done every 2 hours. Samples were decimally diluted in PPS and appropriate dilutions were plated on BHI agar. For the mixed cultures, an additional $1 \mathrm{ml}$ sample was taken at each time point, centrifuged for $10 \mathrm{~min}$ at $5000 \mathrm{xg}$ and the pellet was frozen at $-20^{\circ} \mathrm{C}$ until DNA extraction and PCR quantification of WT and variant 14 as described above. Validations were done in independent duplicates. Model performance was evaluated by determining the accuracy factors $\left(\mathrm{A}_{\mathrm{f}}\right)$ and bias factors $\left(\mathrm{B}_{\mathrm{f}}\right)$ as introduced 
by Ross et al. [31]. Although originally developed to assess the performance of models predicting generation times, in this study we used $A_{\mathrm{f}}$ and $\mathrm{B}_{\mathrm{f}}$ to evaluate predicted counts against observed counts as done previously [32-34]:

$$
\begin{aligned}
& A_{f}=10^{\sum \log \left(\log \left(N_{t}\right)_{\text {predicted }} / \log \left(N_{t}\right)_{\text {observed }}\right) / n} \\
& B_{f}=10^{\left.\sum \log \left(\log \left(N_{t}\right)\right)_{\text {redicticed }} / \log \left(N_{t}\right)_{\text {observed }}\right) / n}
\end{aligned}
$$

Bias factors $>1$ are caused by predicted values higher than the observed values and bias factors $<1$ are caused by predicted values lower than the observed values [31]. In the case of evaluating predicted $\log _{10}\left(\mathrm{~N}_{\mathrm{t}}\right)$ against measured $\log _{10}\left(\mathrm{~N}_{\mathrm{t}}\right)$, bias factors $<1$ give a fail-dangerous prediction. $A_{\mathrm{f}}$ and $\mathrm{B}_{\mathrm{f}}$ were calculated on the average growth prediction $\left(\mu_{\max } \cdot \lambda=2\right.$ and $\left.\mathrm{N}_{\max }=9.5\right)$.

\section{Chain analysis}

Behavior of the WT and variant 14 was also evaluated in a chain of growth and inactivation conditions. The principle was to simulate a milk pasteurization chain, followed by cooled storage and low $\mathrm{pH}$ exposure, simulating stomach passage, adjusted in such a way that the complete chain could be validated under laboratory conditions, in singleand mixed cultures, takinginto account the detection limitations. The chain therefore consisted of the following steps: growth at $7^{\circ} \mathrm{C}$ for 4 days (step 1), a heat inactivation step of $10 \mathrm{sec}$ at $61^{\circ} \mathrm{C}$ (step 2), growth at $7^{\circ} \mathrm{C}$ for 3 days (step 3 ), growth at $10^{\circ} \mathrm{C}$ for 4 days (step 4) and an acid inactivation step of 4 min at $\mathrm{pH}$ 2.5 (step 5). Predictions for growth were made using the gamma model, based on the estimated cardinal growth parameters of WT and variant 14 in Table 5.1. Three scenarios were chosen, namely no lag time, a theoretical maximum lag time $\left(\mu_{\max } \cdot \lambda=\right.$ 4) and an average lag time $\left(\mu_{\max } \cdot \lambda=2\right)$. Predictions for inactivation were made using the D- and z-values and inactivation kinetics as described in Table 5.2. Only lateexponential phase heat resistance was considered, since the predictions indicated all cells to be still in exponential phase at the moment of heat inactivation. For the acid inactivation step both late-exponential and stationary phase were included in the model prediction (Table 5.3). Growth steps (1, 3 and 4) were all performed in Erlenmeyer flasks containing $50 \mathrm{ml}$ BHI-M in a shaking incubator (200 rpm), set at the correct temperature. Samples were taken once per day for plating and RTPCR quantification as described above. From step 3 on (after heat inactivation) the samples for DNA extraction were treated with Propidium monoazide (PMA) before freezing to prevent amplification of DNA of dead cells. PMA treatment was done as described by Pan et al. [35]. Briefly, $2.5 \mu \mathrm{l} 20 \mathrm{mM}$ PMA solution was added to $1 \mathrm{ml}$ cell culture. This mixture was incubated in the dark at room temperature for $5 \mathrm{~min}$. Subsequently, the tubes were exposed to light in a PMA-Lite LED Photolysis Device 
(Biotium) for 15 min to cross-link the PMA to the DNA. The PMA treatment was repeated another time since it was shown that double PMA treatment gave optimal inhibition of the PCR amplification [35]. After the second PMA treatment the cells were centrifuged for $10 \mathrm{~min}$ at $5000 \mathrm{xg}$ and the pellet was stored at $-20^{\circ} \mathrm{Cuntil}$ DNA extraction. Heat inactivation was done as follows; the complete culture $(50 \mathrm{ml})$ was centrifuged ( $5 \mathrm{~min} 2880 \mathrm{xg}$ ), resuspended in $1 \mathrm{ml} \mathrm{BHI-M}$ and centrifuged again (5 min $2880 \mathrm{x} \mathrm{g}$ ). The pellet was resuspended in $100 \mathrm{\mu l}$ BHI-M and transferred into a $200 \mu \mathrm{l} \mathrm{PCR}$ tube. The heat inactivation was done in a PCR machine set at $61^{\circ} \mathrm{C}$ for $10 \mathrm{sec}$, after which the sample was cooled to $7^{\circ} \mathrm{C}$. When the tubes reached $7^{\circ} \mathrm{C}$ the content was transferred to $50 \mathrm{ml} \mathrm{BHI}$, precooled to $7^{\circ} \mathrm{C}$, a sample was taken for plating and the flask was placed in a $7^{\circ} \mathrm{C}$ shaking incubator (step 3). Acid inactivation (step 5) was done as the acid inactivation experiments described above. Samples were taken after $2 \mathrm{~min}$ and $4 \mathrm{~min}$ exposure to $\mathrm{pH} 2.5$ and corrected for volume. Validations were done in independent duplicates. The limitations for this method were that the counts could only be quantified within the dynamic range of the qPCR $\left(\sim 4.5-9.5 \log _{10} \mathrm{cfu} / \mathrm{ml}\right)$ and the PMA treatment only allowed a maximum of $6 \log _{10}$ $\mathrm{cfu} / \mathrm{ml}$ of dead cells to be present within at least $4 \log _{10} \mathrm{cfu} / \mathrm{ml}$ of viable cells [35]. These limits were experimentally confirmed (data not shown). Model performance for each step and the overall chain was evaluated by determining the accuracy factors $\left(\mathrm{A}_{\mathrm{f}}\right)$ and bias factors $\left(\mathrm{B}_{\mathrm{f}}\right)$ as described above.

\section{Variant fractions within WT population}

The heat and acid inactivation kinetics of the WT and variant 14 were used to calculate the theoretical fraction of rpsU variants within the WT population during stress exposure. This was done as described by van Boeijen et al. [11]. The fraction of rpsU variants was then calculated at each time point (t) using equation 17 :

$$
f_{t, r p s U}=\frac{N_{t, r p s U}}{N_{t, t o t a l}}
$$

The fraction $\left(f_{t, r p s U}\right)$ is equal to the probability to isolate an rpsU variant at this time point. Due to their phenotypic clustering [12], it was assumed that the $11 \mathrm{rpsU}$ variants all show the same inactivation kinetics. The initial fraction of rpsU variants in the first step of the chain was assumed to be $5 \cdot 10^{-7}[36]$. 


\section{Results}

\section{Growth under mild stress conditions}

Cardinal growth parameters were estimated based on the $\mu_{\max }$ values obtained at different temperatures, $\mathrm{pH}$ values and water activity values. The data and model fittings are shown in Figure 5.1 for the WT and variant 14 and for the other variants in Figure S5.1. From these figures and the parameter estimates in Table 5.1, it can be seen that the different variants showed distinct behavior under different environmental conditions. TherpsU variants (14, 15 and 23) showed a growth defect, which was more pronounced closer to the lower temperature growth limits, whereas the same variants have similar growth rates as the WT at the lower limits of growth for $\mathrm{pH}$ and $\mathrm{a}_{\mathrm{w}}$ at $30^{\circ} \mathrm{C}$. Variant 9, 12 and 13 showed a lower $\mu_{\max }$ over the complete temperature range tested, with the most dramatic shift observed for variant 9 .
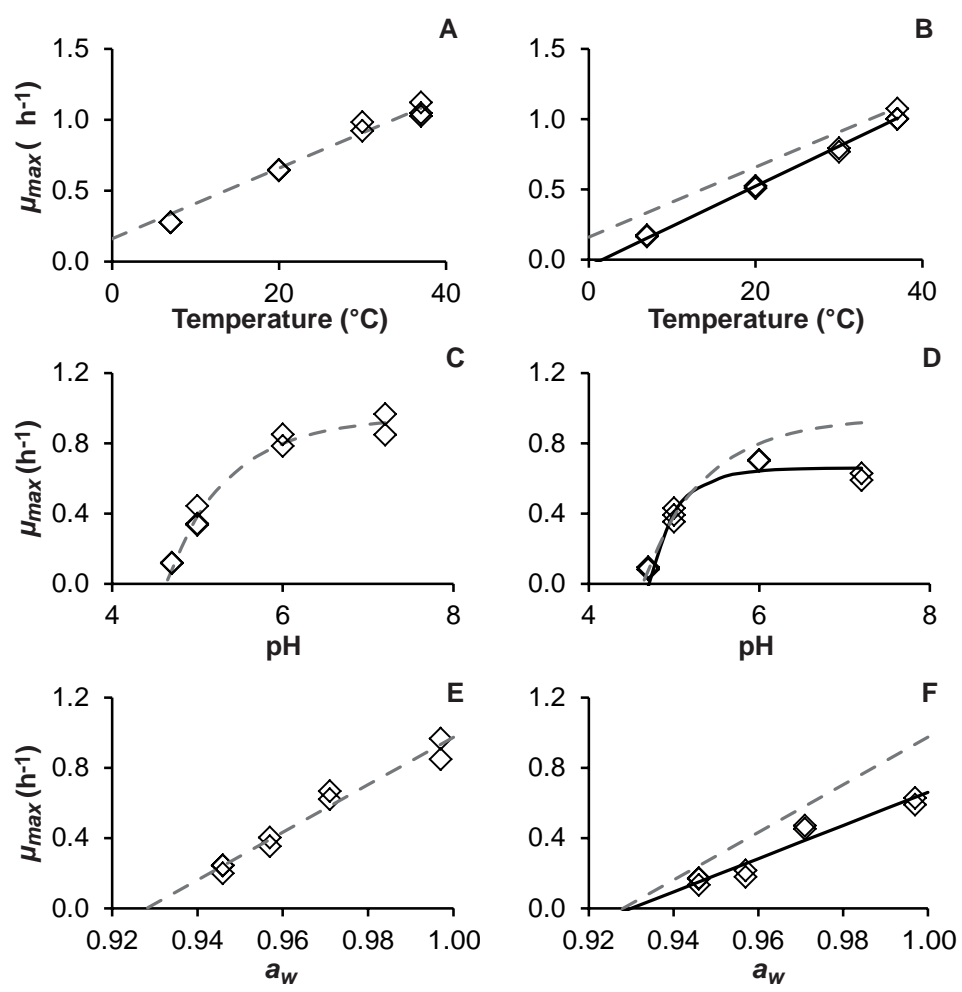

Figure 5.1: Maximum specific growth rate $\left(\mathrm{h}^{-1}\right)$ of $\mathrm{WT}(\mathrm{A}, \mathrm{C}$ and $\mathrm{E})$ and acid resistant variant 14 (B, D and $\mathrm{F}$ ) under different temperature $(\mathrm{A}, \mathrm{B}), \mathrm{pH}(\mathrm{C}, \mathrm{D})$ and $\mathrm{a}_{\mathrm{w}}(\mathrm{E}, \mathrm{F})$ conditions in $\mathrm{BHI}$ (reference condition: $30^{\circ} \mathrm{C}, \mathrm{pH} 7.3, \mathrm{a}_{\mathrm{w}}$ 0.997). Cardinal growth parameters were estimated with the secondary growth models in equation 1-3 (--- WT, - variant 14). 


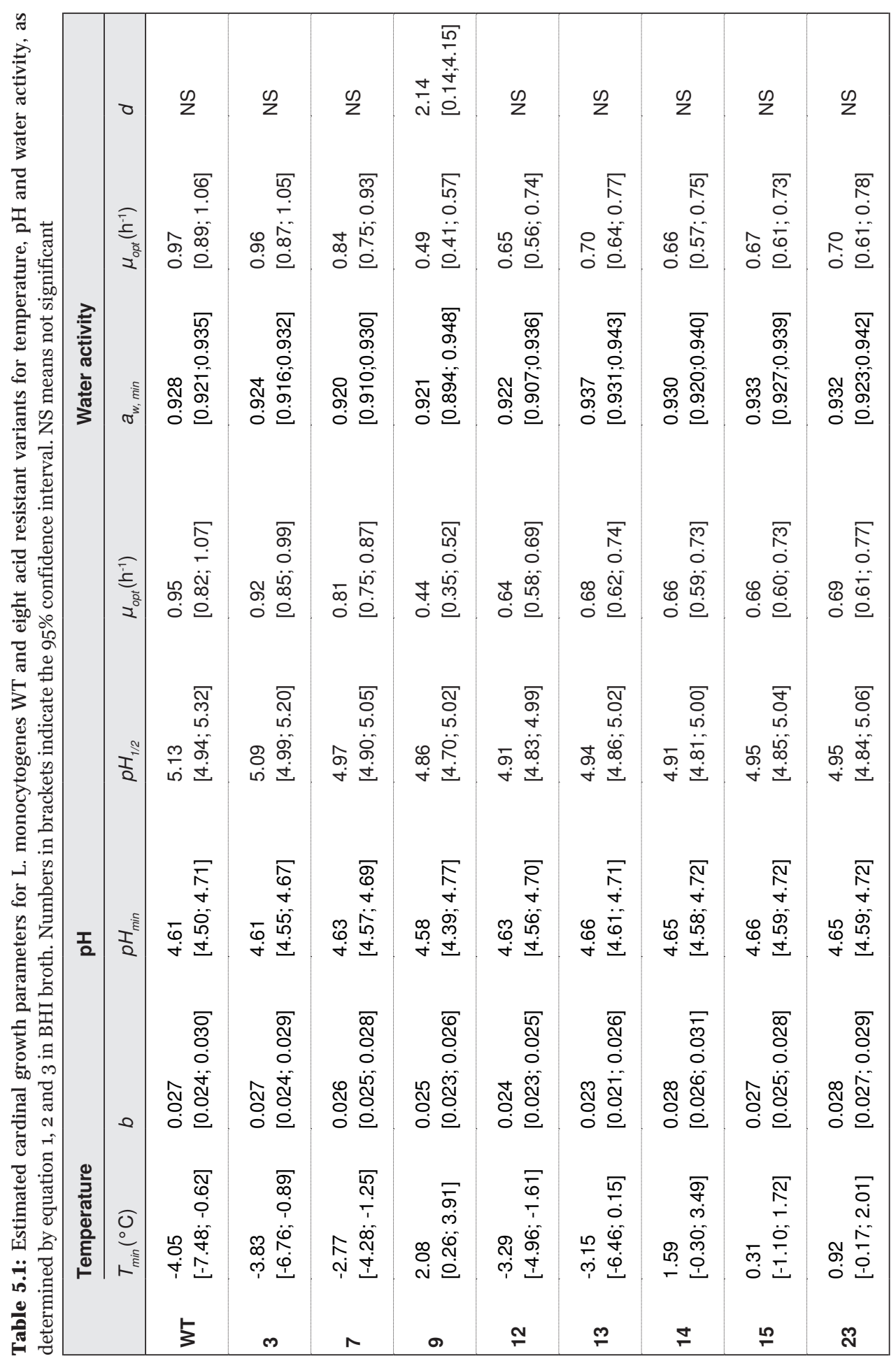


However, also this variant, while growing extremely slow under optimal $\mathrm{pH}$ conditions, had a similar $\mathrm{pH}_{\min }$ to the WT and an even lower minimal estimated $\mathrm{a}_{\mathrm{w}, \min }$ than the WT. For all variants the shape parameter (d) in the $\mathrm{a}_{\mathrm{w}}$ model was not significant and could be excluded according to the lack-of-fit test. Variant 9 was the only variant for which the shape parameter could not be excluded since this would have led to an unrealistic $\mathrm{a}_{\mathrm{w} \text {, min }}$ estimate of $\sim 0.88$. It was experimentally confirmed that variant 9 was not able to grow at such low $\mathrm{a}_{\mathrm{w}}$ and therefore the $\mathrm{d}$ parameter was included, despite not being significant. Variant 3 showed similar behavior to the WT under all growth conditions tested. Variant 7 has a slight growth defect under optimal conditions compared to the WT, but became similar to the WT when the conditions were more stressful.

\section{Growth predictions and validations}

The cardinal growth parameters were used to predict the growth of the WT and eight variants under combinations of mild stresses. From Figure S5.2 it can be seen that depending on the combination of environmental conditions, the variants performed differently. In the milk conditions (Figure S5.2A) the rpsU variants and variant 9 were the slowest growers, with the low temperature contributing mostly to the gamma factor. In the ham condition, the temperature is still the most growth limiting factor, however, the low $\mathrm{a}_{\mathrm{w}}$ reduced the grouth rate even further in all cases. The gamma factor for $\mathrm{pH}(\mathrm{pH}$ 6.0) had almost no influence on the predicted growth rate of the variants. Figure $\mathrm{S} 5.2 \mathrm{C}$ shows that when grown at $37^{\circ} \mathrm{C}$ at $\mathrm{pH} 5.2$ there is almost no difference in $\mu_{\max }$ between the variants and WT. This is due to the fact that the $\mathrm{pH}$ is the growth limiting factor, and at this $\mathrm{pH}$ of 5.2 there is no dramatic difference in predicted $\mu_{\max }$ between the WT and variants. The three growth scenarios displayed in Figure S5.2 were validated, both in single and in mixed cultures of WT and variant 14.

As can be seen from Figure 5.2A, the counts in single and mixed cultures for the WT in BHI-M were well in accordance with the predictions in BHI-M, which was confirmed by a $B_{f}$ of 1.02 . Variant 14 however, showed to have a slightly higher $\mu_{\max }$ than predicted resultingin a relatively low $\mathrm{B}_{\mathrm{f}}$ of 0.80 and an high $\mathrm{A}_{\mathrm{f}}$ of 1.25 , indicating a systematic deviation from the prediction. Both variant and WT did not seem to have an obvious lag time. In mixed cultures of WT and variant, the WT PCR counts were similar to the total plate counts, indicating that the WT was the dominating population. Variant 14 followed the same growth as in singleculture up to 150 hours, after which growth was arrested. This growth inhibition coincides with the entry into stationary phase of the WT. In BHI-H both WT and variant counts matched the prediction well in single culture (Figure $5.2 \mathrm{~B}$ ) and $\mathrm{B}_{\mathrm{f}}$ was 1.04 and 0.94 respectively. 


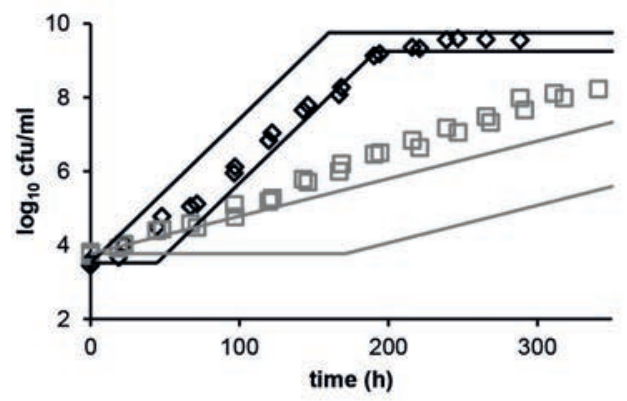

A1

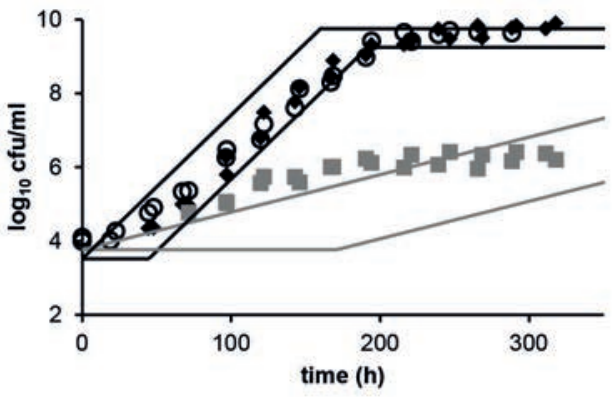

A2

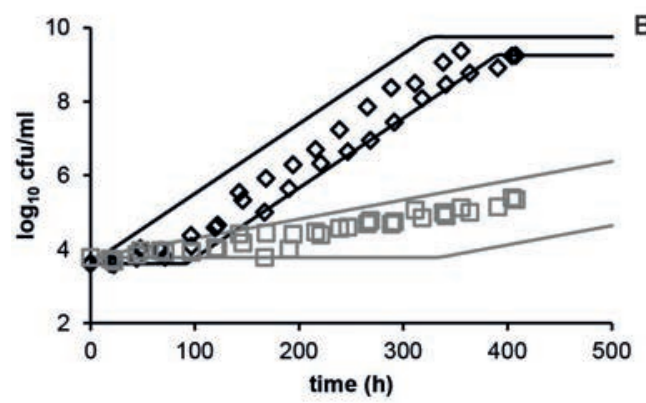

B1
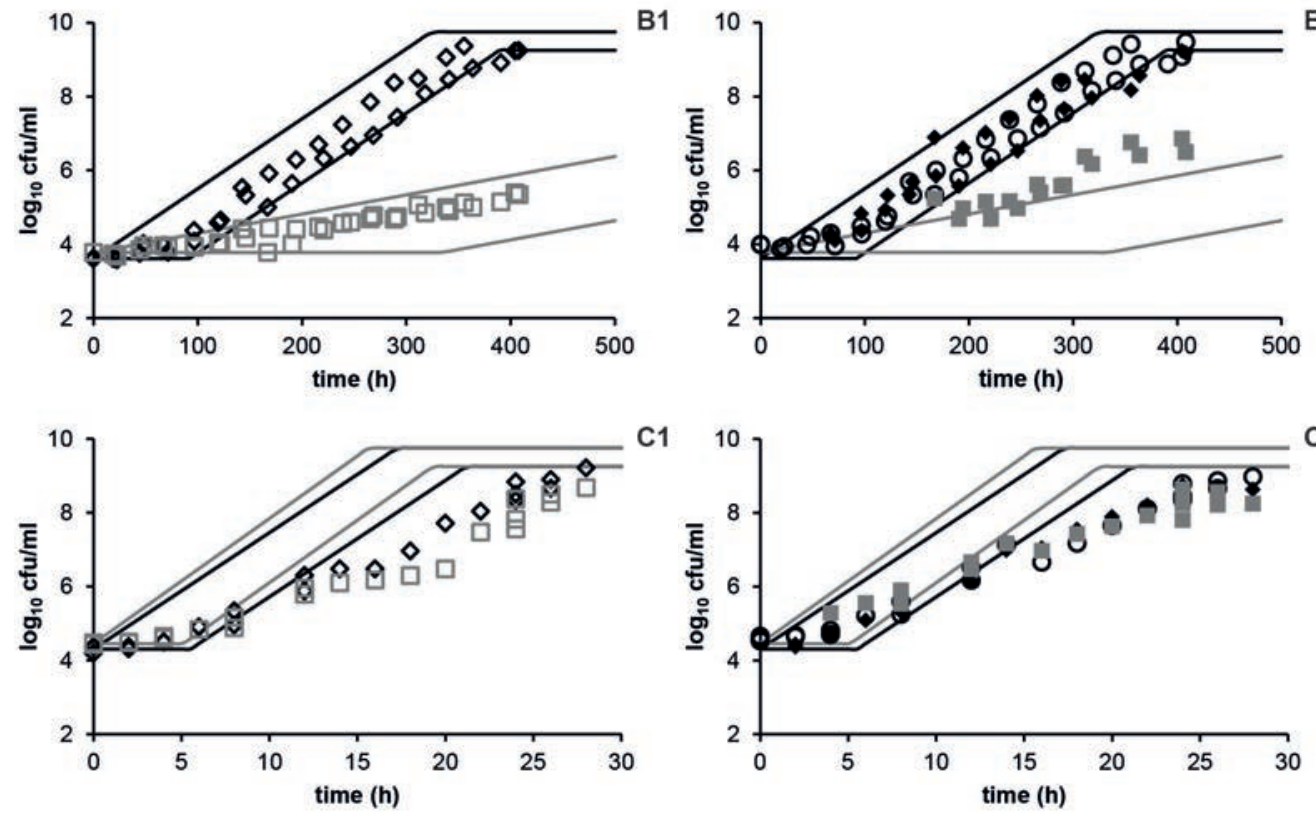

C1

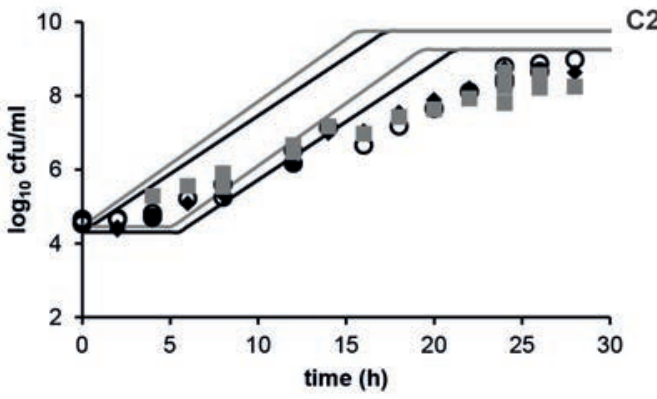

Figure 5.2: Growth predictions and validations for L. monocytogenes LO28 WT (black, counts diamonds) and acid resistant variant 14 (grey, counts squares). Growth predictions according to the three-phase linear model were based on cardinal growth parameters as shown in Table 5.1 and the gamma model. 3 scenarios were considered: milk (BHI-M, pH 6.6; $\left.\mathrm{a}_{\mathrm{w}} 0.997 ; 7^{\circ} \mathrm{C}\right)(\mathrm{A})$, ham (BHI-B, $\mathrm{pH} 6.0 ; \mathrm{a}_{\mathrm{w}} 0.965 ; 7^{\circ} \mathrm{C}$ ) (B) and $\mathrm{BHI}$ at $\mathrm{pH} 5.2$ at $37^{\circ} \mathrm{C}$ (BHI-S) (C). For each scenario two situations were considered, namely no lag phase (upper line) and a maximum lag phase of $\mu \cdot \lambda=4$ (lower line). Validations were done by plate counts in single cultures (1) and mixed cultures (2). For the mixed cultures, total plate counts (circles) are displayed by the open symbols and individual contributions of WT and variant 14 in the mixture were deducted from $\mathrm{c}_{\mathrm{t}}$ values and the standard curves correlating $\mathrm{c}_{\mathrm{t}}$ values to $\log \mathrm{cfu} / \mathrm{ml}$ counts (closed symbols).

Interestingly, the growth of the WT matched the prediction with the maximum lag time, whereas the variant did not seem to have a lag time at all. Another interesting observation is that when mixed with the WT, variant 14 seemed to perform better than when being cultured alone, as indicated by higher counts in mixed cultures than in single culture. In BHI-S cultures, the predictions suggested the variant and WT to 
grow at similar speed (Figure 5.2C). This was confirmed by the growth validations, both in single and mixed cultures, although both grew slower than predicted. Both WT and variant seemed to have a lag phase, most likely caused by the transition from $\mathrm{pH} 7.3$ of the ON culture to $\mathrm{pH} 5.2$ of the BHI-S. Bias factors of 1.11 for the WT and 1.18 for the variant also indicated that both predictions are a slight overestimation.

\section{Stress resistance}

Heat resistance was evaluated in late-exponential and stationary phase for the WT and variant 14 at 55, 60 and $62{ }^{\circ} \mathrm{C}$. As can be seen from Figure 5.3, the inactivation curvatures were different, depending on the temperature and growth phase tested. This resulted in different inactivation models giving the best fit, making comparison and especially the calculation of D- and z-values difficult. Because the major part of the reduction range is most important for the validation experiments, it was decided to also fit an inactivation model to the data without considering the tail. Either the 5D-Weibull model (Eq. 13) or the linear inactivation model (Eq. 11) could be fitted to all the inactivation curves, allowing for D-value estimation based on the major part of the reduction range (Table 5.2). Corresponding z-values were 5.7 and 4.0 for WT and variant respectively in late-exponential phase and 4.4 and 5.7 for WT and variant respectively in stationary phase.

Table 5.2: Inactivation parameter estimates $\mathrm{D}(\mathrm{min})$ and $\beta$ upon heat exposure by fitting the reparametrized 5D-Weibull model to the data without taking the tail into consideration. For the Weibull model the values of $\mathrm{D}$ were obtained by dividing $\mathrm{t}_{\mathrm{SD}}$ by 5 . The linear model was used when the shape parameter was not significantly different from 1 , the F-test confirmed a statistically acceptable fit of the linear model and the lack-of-fit test confirmed that the shape parameter could be removed. In all other cases the Weibull model was used. NS indicates that the linear model was fitted and therefore an estimate for b was not applicable. The $95 \%$ confidence interval of the parameter estimates is indicated in brackets.

\begin{tabular}{|c|c|c|c|c|c|c|c|}
\hline & & \multicolumn{3}{|c|}{ Late-exponential phase } & \multicolumn{3}{|c|}{ Stationary phase } \\
\hline & & $55^{\circ} \mathrm{C}$ & $60^{\circ} \mathrm{C}$ & $62^{\circ} \mathrm{C}$ & $55^{\circ} \mathrm{C}$ & $60^{\circ} \mathrm{C}$ & $62^{\circ} \mathrm{C}$ \\
\hline WT & $D$ & $\begin{array}{l}1.92 \\
{[1.73 ; 2.15]}\end{array}$ & $\begin{array}{l}0.28 \\
{[0.24 ; 0.33]}\end{array}$ & $\begin{array}{l}0.11 \\
{[0.08 ; 0.17]}\end{array}$ & $\begin{array}{l}24.0 \\
{[21.4 ; 27.3]}\end{array}$ & $\begin{array}{l}1.17 \\
{[1.04 ; 1.33]}\end{array}$ & $\begin{array}{l}0.71 \\
{[0.63 ; 0.79]}\end{array}$ \\
\hline & $\beta$ & NS & NS & NS & NS & NS & $\begin{array}{l}1.59 \\
{[0.98 ; 2.21]}\end{array}$ \\
\hline 14 & $D$ & $\begin{array}{l}15.7 \\
{[14.6 ; 16.9]}\end{array}$ & $\begin{array}{l}0.65 \\
{[0.63 ; 0.68]}\end{array}$ & $\begin{array}{l}0.32 \\
{[0.28 ; 0.39]}\end{array}$ & $\begin{array}{l}29.9 \\
{[28.8 ; 30.9]}\end{array}$ & $\begin{array}{l}2.70 \\
{[2.42 ; 3.05]}\end{array}$ & $\begin{array}{l}1.97 \\
{[1.92 ; 2.02]}\end{array}$ \\
\hline & $\beta$ & $\begin{array}{l}2.06 \\
{[1.49 ; 2.63]}\end{array}$ & $\begin{array}{l}1.73 \\
{[1.45 ; 2.00]}\end{array}$ & NS & $\begin{array}{l}2.43 \\
{[1.96 ; 2.90]}\end{array}$ & NS & $\begin{array}{l}1.46 \\
{[1.35 ; 1.58]}\end{array}$ \\
\hline
\end{tabular}



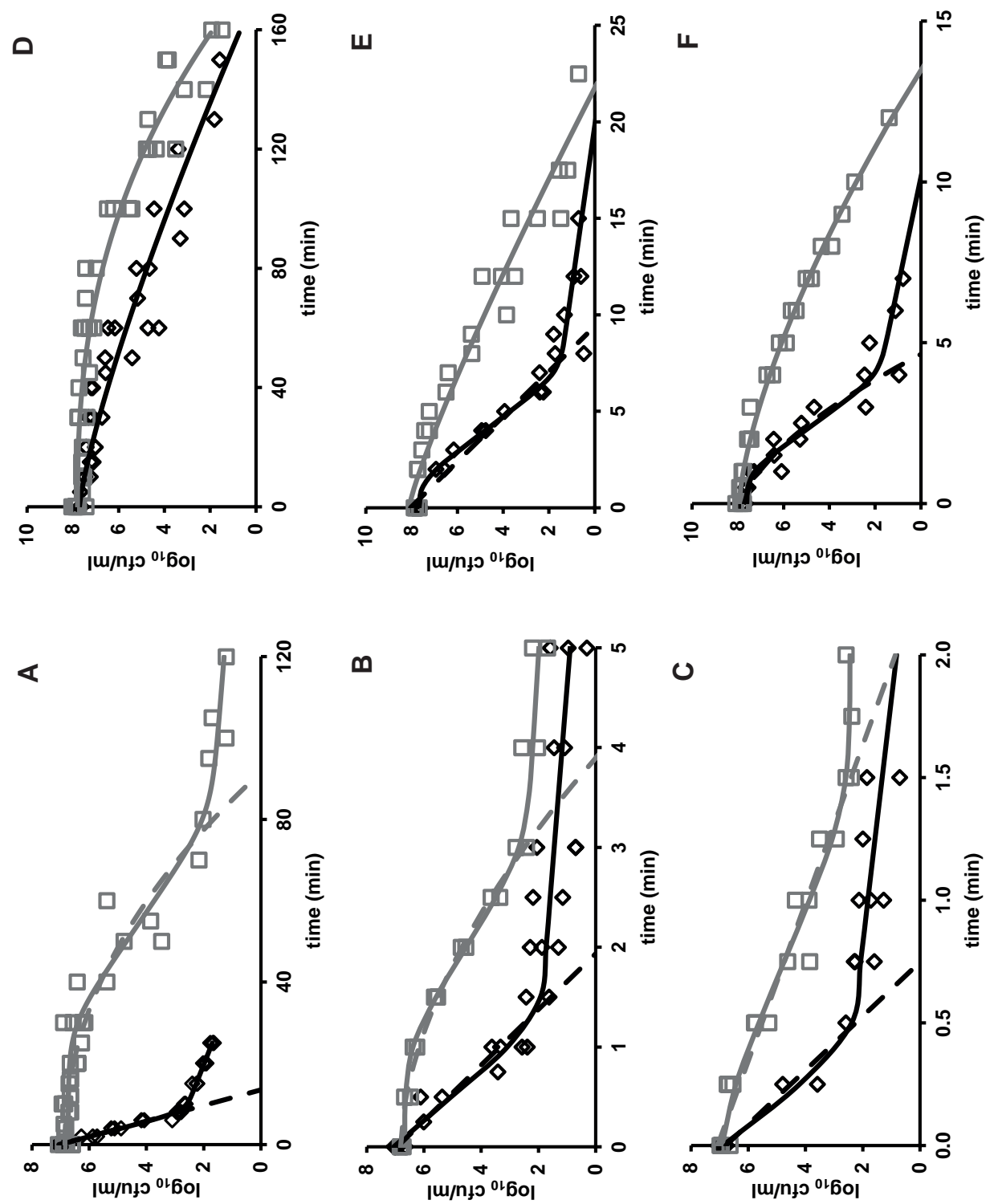

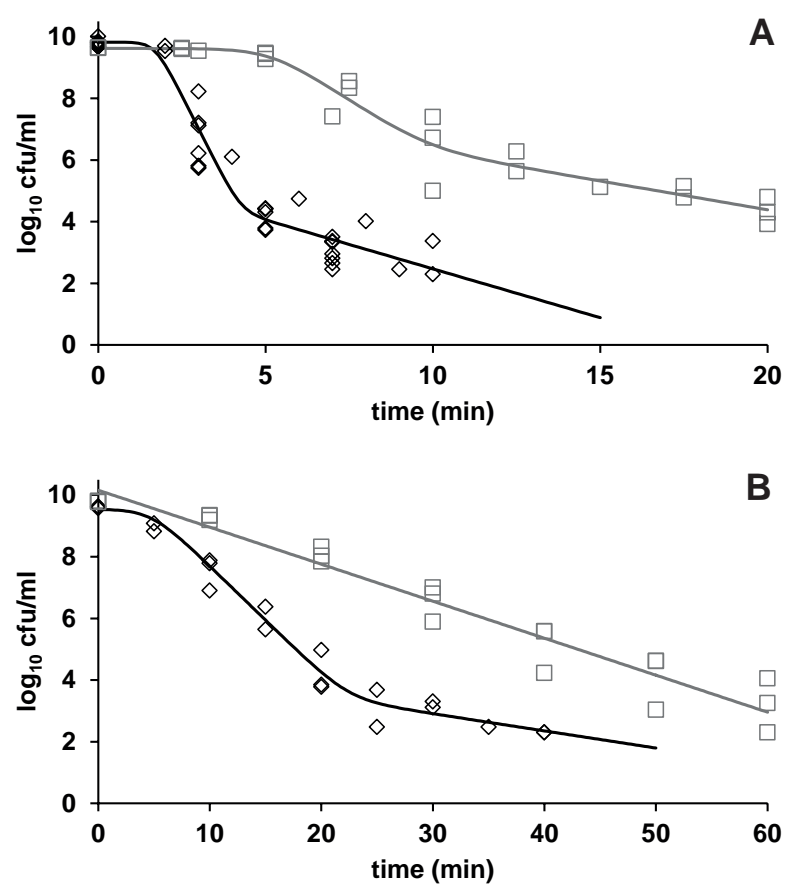

Figure 5.4: Acid inactivation kinetics of L. monocytogenes LO28 WT (black) and acid resistant variant 14 (grey) in $\mathrm{BHI}$ set at $\mathrm{pH} 2.5$ by $10 \mathrm{M} \mathrm{HCl}$ in late-exponential phase (A) and stationary phase (B) at $37^{\circ} \mathrm{C}$. Biphasic, linear model with shoulder or linear model have been fitted to the inactivation data (solid line) depending on the best fitting model.

Both fits, with and without tail, can be seen in Figure 5.3. Whereas in late-exponential phase the difference in heat resistance between WT and variants became smaller with higher temperatures, the difference between WT and variant was smaller at lower temperatures in stationary phase. This was also reflected by a higher z-value in stationary phase for the variant compared to the WT and a higher z-value for the WT in late-exponential phase. Also for acid inactivation (Figure 5.4) there was a clear difference between WT and variant 14, as well as a clear difference between lateexponential and stationary phase. Both in late-exponential and stationary phase, the increased resistance of the variant was characterized by a longer shoulder period and a lower inactivation rate (see Table 5.3). 
Table 5.3: Inactivation parameter estimates of $\mathrm{LO} 28 \mathrm{WT}$ and variant 14 upon exposure to BHI set to $\mathrm{pH}$ 2.5 by $10 \mathrm{M} \mathrm{HCl}$ at $37^{\circ} \mathrm{C}$ in late-exponential or stationary phase by fitting the biphasic model with shoulder or linear model to the data

\begin{tabular}{|llll|}
\hline & Parameter & Late-exponential phase & Stationary phase \\
\hline WT & $N_{o}\left(\log _{10} \mathrm{cfu} / \mathrm{ml}\right)$ & $9.8[9.4 ; 10.3]$ & $9.5[9.1 ; 10.0]$ \\
& $k_{\text {sens }}\left(\mathrm{min}^{-1}\right)$ & $4.98[2.66 ; 7.30]$ & $0.81[0.64 ; 0.98]$ \\
& $k_{\text {res }}\left(\mathrm{min}^{-1}\right)$ & $0.73[0.25 ; 1.21]$ & $0.13[-0.03 ; 0.29]$ \\
& Shoulder $(\mathrm{min})$ & $1.7[1.0 ; 2.4]$ & $4.8[2.2 ; 7.4]$ \\
& Resistant fraction & $10^{-4.72}\left[10^{-5.92} ; 10^{-3.52}\right]$ & $10^{-5.22}\left[10^{-7.22} ; 10^{-3.22}\right]$ \\
& $N_{o}\left(\log _{10} \mathrm{cfu} / \mathrm{ml}\right)$ & $9.6[9.1 ; 10.1]$ & $10.2[9.7 ; 10.6]$ \\
& $k_{\text {sens }}\left(\mathrm{min}^{-1}\right)^{\mathrm{a}}$ & $1.66[0.35 ; 2.97]$ & $0.27[0.25 ; 0.31]$ \\
& $k_{\text {res }}\left(\mathrm{min}^{-1}\right)$ & $0.43[0.12 ; 0.74]$ & \\
& Shoulder $(\mathrm{min})$ & $5.2[3.0 ; 7.4]$ & \\
& Resistant fraction & $10^{-2.46}\left[10^{-4.10} ; 10^{-0.82}\right]$ & \\
\hline
\end{tabular}

\section{Probability predictions}

The inactivation kinetics were used to estimate the fraction of the rpsU variant within the WT population at any given time during stress exposure. The probability of isolating an rpsU variant was evaluated under different stress conditions. To do so, the inactivation kinetics displayed in Figure 5.3 and 5.4 were used. The initial rpsU variant fraction in an exponentially growing culture was determined as described by van Boeijen et al. [11] and estimated to be $\sim 5 \cdot 10^{-7}$ [36]. Two different scenarios are shown in Figure 5.5. Figure 5A shows the probability that a heat treatment of $55^{\circ} \mathrm{C}$ would have resulted in isolating an rpsU variant. This probability increases over time and has a maximum probability of $\sim 11 \%$ after $30 \mathrm{~min}$. At this time point however, the total population is already quite small with around $100 \mathrm{cfu} / \mathrm{ml}$. Exposure to $\mathrm{pH}$ 2.5 (Figure $5.5 \mathrm{~B}$ ) gave a different distribution and it can be seen that the probability of isolating variants at $\mathrm{pH} 2.5$ had a peak distribution. These data showed that the probability of isolating a specific variant highly depends on the inactivation kinetics of both variant and WT, the specific process conditions, as well as the initial variant fraction within the WT population. 

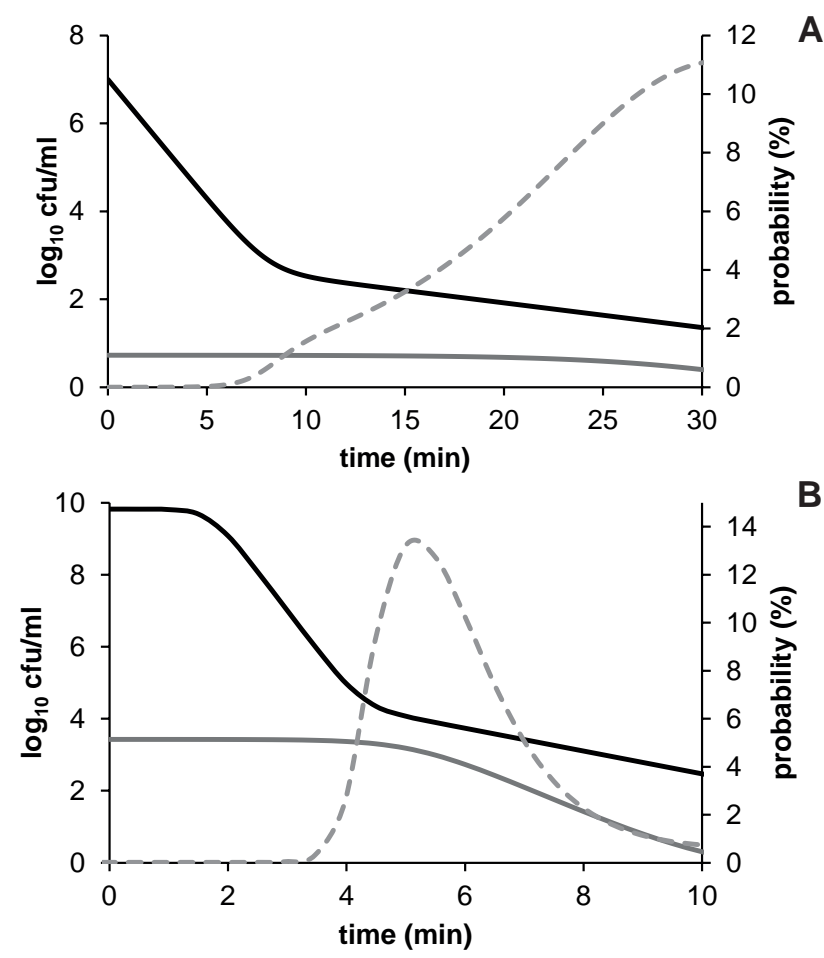

Figure 5.5: Probability of isolating rpsU variants upon heat and acid inactivation of late-exponential phase cells at $55^{\circ} \mathrm{C}(\mathrm{A})$ and $\mathrm{pH} 2.5$ (B). The initial fraction of rpsU variants within a late-exponential phase culture was estimated to be $5.2 \cdot 10^{-7}[36]$. This fraction, combined with the inactivation kinetics of the WT (black line) and rpsU variant 14 (grey line), was used to determine the rpsU variant fraction (dashed line) at each time point during the inactivation curve according to the method of Van Boeijen et al. [11].

\section{Chain analysis and validation}

The growth and inactivation data were combined to make predictions about the behavior of the WT and variant 14 along a simulated model food chain. Due to technical limitations of the experimental set-up, the different processing steps were adjusted according to the detection limitations. After each step in the chain, the predicted $\mathrm{N}_{0}$ was adjusted to the measured $\mathrm{N}_{0}$ for visualization purposes. From Figure 5.6 it can be seen that generally higher growth rates and higher inactivation rates were predicted for the WT compared to variant 14. In the first growth step, the WT data match very well with the prediction, resulting in $\mathrm{B}_{\mathrm{f}}$ of 1.00 . The numbers for the variant however, are higher than predicted, which leads to a $\mathrm{B}_{\mathrm{f}}$ of 0.85 in the first step. This is mostly due to the predicted lag time which is not apparent. In Figure S5.4, also the prediction without lag time is considered and it can be seen that in this case also the variant counts are in accordance to the prediction. The first heat inactivation step gave a much higher inactivation than predicted for the WT. 

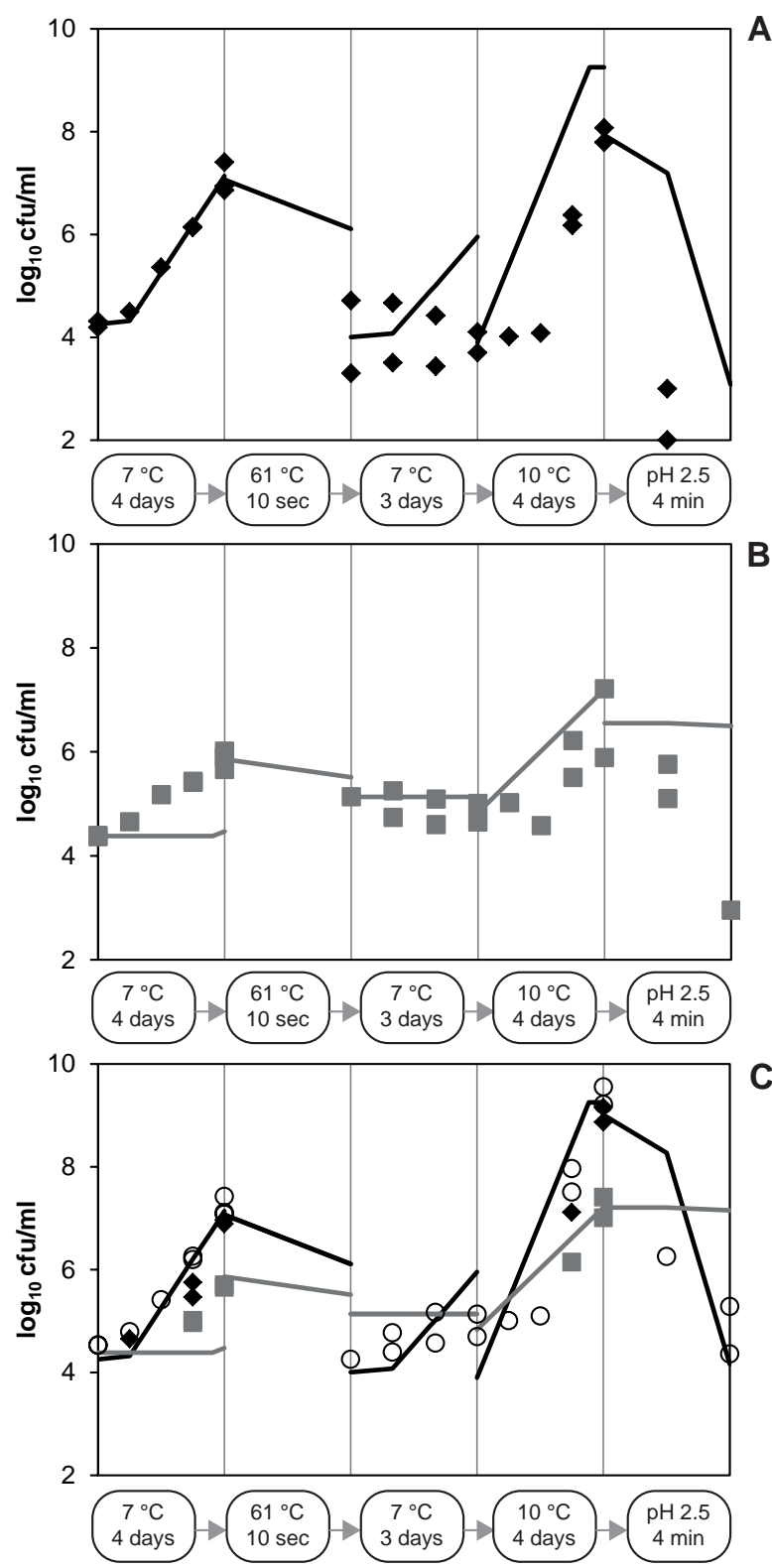

Figure 5.6: Performance of WT (black) and variant 14 (grey) along a model food chain. The chain was simulated in BHI-M (pH 6.6). Growth and inactivation behavior was predicted (solid lines) based on growth and inactivation kinetics as shown in Table 5.1-5.3 and Figure 5.1, 5.3 and 5.4. Predictions were validated in $\mathrm{BHI}$ set to $\mathrm{pH} 6.6$ by $10 \mathrm{M} \mathrm{HCl}$, simulating milk characteristics. In the first growth step an average lag time of $\mu \cdot \lambda=2$ was chosen and in the growth step after heat treatment a maximum lag time of $\mu \cdot \lambda=4$. Stress resistance predictions were based on late-exponential phase cells as stationary phase was not reached before. Validations were done in single cultures (A: WT, B: variant 14) and in mixed cultures (C). Open symbols represent the plate counts and closed symbols the individual contributions of WT (black) and variant 14 (grey) in the mixture as deducted from $\mathrm{c}_{\mathrm{t}}$ values and the standard curves correlating $\mathrm{C}_{\mathrm{t}}$ values to $\log \mathrm{cfu} / \mathrm{ml}$ counts. 
Also the subsequent predicted lag time $(\lambda \cdot \mu=4)$ did not seem sufficiently long as both WT and variant 14 needed a longer recovery time to resume grouth after the heat treatment. The acid inactivation step gave a larger reduction in viable numbers than predicted, especially for the WT but also for the variant.

Overall, the general trend of better growth for the WT and higher stress resistance for the variant was clearly confirmed along this model food chain, although the plate counts did not match the predictions very well for every step along the chain model. The bias factors indicated a better overall fit for variant 14 than for the WT (Table 5.4). In the mixed culture (Figure 5.6C), the trend was generally the same as in single cultures, but also some differences were observed. Due to the technical limitations of the qPCR quantification and PMA method, the counts for WT and variant could only be obtained when plate counts were higher than $6 \log _{10} \mathrm{cfu} / \mathrm{ml}$ after heat treatment.

Table 5.4: Bias factors $\left(B_{\mathrm{f}}\right)$ and accuracy factors $\left(A_{\mathrm{f}}\right)$ for WT and variant 14 along a model food chain. The chain was simulated in BHI-M (see Figure 5.6). Validations for each step were done in BHI-M for WT and variant 14. Bias factors $\left(\mathrm{B}_{\mathrm{f}}\right)$ and accuracy factors $\left(\mathrm{A}_{\mathrm{f}}\right)$ were calculated for each step separately and for the complete chain based on daily measurements

\begin{tabular}{|lcccc|}
\hline & \multicolumn{2}{c}{ WT } & \multicolumn{3}{c|}{ Variant 14 } \\
\cline { 2 - 5 } Step & $A_{f}$ & $B_{f}$ & $A_{f}$ & $B_{f}$ \\
\hline $7^{\circ} \mathrm{C}, 4$ days & 1.02 & 1.00 & 1.18 & 0.85 \\
$61^{\circ} \mathrm{C}, 10 \mathrm{sec}$ & 1.55 & 1.55 & 1.07 & 1.07 \\
$7^{\circ} \mathrm{C}, 3$ days & 1.20 & 1.11 & 1.06 & 1.05 \\
$10^{\circ} \mathrm{C}, 4$ days & 1.35 & 1.35 & 1.14 & 1.14 \\
$\mathrm{pH} 2.5,2$ min & 2.94 & 2.94 & & \\
$\mathrm{pH} 2.5,4$ min & & & 1.48 & 1.48 \\
total & 1.28 & 1.24 & 1.17 & 1.04 \\
\hline
\end{tabular}

Despite this limitation, faster growth for the WT than variant 14 could be confirmed. When the cells started growing again after the heat treatment, the WT in the mixed culture reached higher numbers than the WT in single culture. This indicated that either the WT was still present in higher numbers after the heat treatment in mixed culture compared to single culture, or the WT cells grew faster in mixed culture than in single culture. At the end of the 'single culture' chain, more cells were present of variant 14 than the WT. At the end of the chain in the mixed culture, there was a significant amount of cells left and the reduction was similar to the reduction observed for variant 14 . However, the amount of dead cells in the culture was too high ( $>6 \log _{10} \mathrm{cfu} / \mathrm{ml}$ ) to confirm that the surviving cells were either WT or variant 14, as the PMA method does not correctly distinguish dead cells from living cells when more than $6 \log _{10} \mathrm{cfu} / \mathrm{ml}$ of dead cells are present. 


\section{Discussion}

This study aimed to quantify the behavior of L. monocytogenes WT and acid resistant variants under different environmental conditions, in order to get more insight into the potential behavior of these variants in a food chain and during stomach passage. The obtained set of fitness and robustness parameters provided more insight in the behavior of the different variants and highlighted the diversity within the L. monocytogenes population. This diversity was already established qualitatively by Metselaar et al. [12], but the current set of parameters provided quantitative data on this behavior, which was subsequently used for prediction of population dynamics under different environmental conditions. Predictions based on cardinal growth parameters and the gamma model confirmed the disadvantage variant 14 has under most growth conditions, but also indicated that there are combinations of mild stress conditions where the growth rate of the variant and the WT were rather similar. The gamma model allowed for prediction of growth under combined mild stress conditions, assuming no interaction between the different growth limiting factors [37]. The gamma theory is, however, not always pertinent and its applicability seems to depend on the organism, as well as on the combination of mild stress factors [26, $38,39]$. Especially around the growth boundaries, the gamma model has shown poor agreement with experimental observations when no synergy factor was included [40]. However, for L. monocytogenes amongst others it was shown that further away from the growth boundaries, the gamma model without synergy generally performs well to describe the combined effect of water activity, $\mathrm{pH}$ and temperature on growth [41]. Also from the growth validations in Figure 5.2 the gamma theory without synergy seems to be valid since the prediction described that data generally well. In BHI-M, the variant even grew faster than predicted (Figure 5.2B). A possible explanation for this deviation from the model could be that the variant has a slightly lower $\mathrm{pH}_{\mathrm{opt}}$ than the WT, which can be observed in Figure S5.1B. pH 7.3 was considered for the WT and all variants and a lower $\mathrm{pH}_{\text {opt }}$ can lead to underestimation of the growth rate around the actual $\mathrm{pH}_{\mathrm{opt}}$. A possibility is that only the mean cardinal parameter estimates were included in the prediction. When the lower and upper $95 \%$ confidence interval of the cardinal parameter estimates were taken into account, the data points fell within the prediction (see supplemental data Figure S5.3). Including this experimental and biological variability, as well as best- and worst case scenarios for the lag time duration, resulted in a very wide prediction interval, but using the upper 95\% limit with no lag time, the prediction can be considered fail-safe. The growth rate at $37^{\circ} \mathrm{C}$ at $\mathrm{pH} 5.2$ was lower than predicted both for the WT and variant, and even when taking the $95 \%$ confidence interval into account the counts were on the lower limit of the prediction. This could be due to the fact that the $\mathrm{pH}$ of 5.2, which is the most growth limiting factor in this scenario, is in the steep part of the curvature 
of the cardinal pH model (see Figure 5.1) and accurate prediction is therefore more proneto errors. In themixed cultures, theWT is generally thedominating population. As expected, the variant reached stationary phase as soon as the WT reached stationary phase, which is most likely due to nutrient limitation. Another interesting observation is that variant 14 performed better in BHI- $\mathrm{H}$ when mixed with the WT than in single culture. This would indicate an interaction between WT and variant in mixed cultures and this is a complicating factor in making accurate predictions of the population dynamics during growth. Prediction of microbial behavior in a sequential chain of growth and inactivation conditions, remains to be a challenge in predictive microbiology. The use of accuracy and bias factors on $\log _{10} \mathrm{cfu} / \mathrm{ml}$ counts instead of generation times does not allow for using the criteria for acceptable or unacceptable model performance, which are commonly used for generation time validations [42]. Nonetheless, these performance indices are useful for getting a quantitative measure of model performance and for comparison of performance of different steps along a model food chain. In this study, the use of predictive modelling, combined with a validation method based on the genetic background of the rpsU variants, proved to providevaluableinformation on the population dynamics of L. monocytogenes under changing environmental conditions, despite the sometimes poor agreement between model predictions and validation. Growth phase and growth history are well-known factors affecting stress resistance of bacterial cells. Many researchers have shown the effect of culturing cells at low temperatures on thermal resistance and the general agreement is that $\mathrm{D}$-values decrease with decreasing preculturing temperatures $[17,18,43,44]$, which is in accordance to our results. Not only temperature, but also other environmental factors, as well as age and growth phase of the culture are shown to affect stress resistance [17, 45]. Aryani et al. [46] showed that the growth phase is the growth history factor affecting the heat resistance to the greatest extent, compared to different temperatures, $\mathrm{pH}$ and salt levels during preculturing. In the chain model predictions, late-exponential phase cells were used, which were even more heat sensitive than the prediction for late-exponential phase cells. This indicates a multiplicative effect on heat sensitivity of growth at low temperature and late-exponential grouth phase. Also the acid inactivation step resulted in a higher reduction than predicted. This is most likely also due to the growth at $7^{\circ} \mathrm{C}$ as Ivy et al. showed that L. monocytogenes is more sensitive to low $\mathrm{pH}$ when grown at $7^{\circ} \mathrm{C}$ compared to 30 or $37^{\circ} \mathrm{C}$, both in exponential and stationary phase [47]. Another factor that was not taken into account in the model, but seems to be a major factor influencing the performance of the model, is the recovery time that is needed after the heat inactivation step. Even the maximum theoretical lag phase (i.e. $\mu \cdot \lambda=4$ ) was not sufficient to describe the recovery time after heat treatment, especially for the WT. It has been well documented that heat treatment leads to sublethal damage of cells and several studies have shown that lag times can vary widely between individual cells 
in a population, and that the variability in the lag time of single cells increases with severity of treatment $[48,49]$. With low cell numbers and severe stress treatments, the lag phase can increase well above the maximum lag time of $\mu \cdot \lambda=4$ that was used in the model chain [50]. Also, the recovery conditions play an important role in the number of viable cells that can be retrieved after lethal stress treatment [51] and the $7^{\circ} \mathrm{C} \mathrm{BHI}-\mathrm{M}$ which was the recovery conditions in the model food chain is not optimal for the cells. The extended time needed for recovery is more pronounced for the WT than for the variant since the lag time was predicted to be shorter for the WT after heat treatment than for the variant, but the validations showed that the recovery times were similar for both types of cells. Like for the growth predictions, only the mean values of the parameter estimations were considered in the chain model, as this was sufficient to get more insight in the behavior along a series of growth and inactivation events. When the aim is to make more realistic and fail-safe predictions, the biological variability, reflected in the $95 \%$ confidence intervals of the cardinal parameter estimates, should also be taken into account.

Many factors are known to decrease thermal resistance of $\mathrm{L}$. monocytogenes, which also seems to be a cause of the overestimation of the numbers of culturable cells in the chain model compared to the prediction. Many food products on the other hand, are known to increase heat resistance of microorganisms [45, 52]. Validating the current chain model in laboratory medium and to take all the different variability factors into account was already challenging; translating such a model to actual food matrices will be even more demanding. Nonetheless, the current chain model gives a good indication of the possible population dynamics along a food chain. For experimental reasons the amount of variant and WT were chosen to be the same in the first step but the actual rpsU variant fraction in a WT population is very small and was shown to be $\sim 5 \cdot 10^{-7}[36]$. The probability that an rpsU variant is present in a batch of food product therefore depends not only on the environmental conditions encountered, but also on the initial contamination level and the batch size. Low contamination levels and small batch size will not likely result in selection for stress resistant variants, simply because the fraction is too low. The variant fraction can also change upon different processing conditions as illustrated in Figure 5.5. On the other hand, small changes in variant fraction and in inactivation kinetics, which are also affected by the growth history, can dramatically affect the chances that certain processing conditions lead to selection of stable resistant variants. Multiple stress cycles may also affect the fraction of variants in the population. Van Boeijen et al. [11] reported that heat resistant variants were not found after single heat treatment due to low probability of finding them, but after a few cycles, the fraction of heat resistant variants increased significantly. Also the presence of L. monocytogenes in biofilms was shown to affect 
the stress resistance of the cells [19]. It is not unlikely that under more stringent heat treatment, the variant can become the dominating population and combined with its slow growth becomes a persister in industrial settings. In the current study, only an rpsU mutant was quantitatively characterized in detail, since this was the only variant for which a mutation was known that allowed for discrimination from the WT by qPCR. As can be seen from Table 5.1, the other types of acid resistant variants show different behavior and are also known to display different degrees of resistance [12]. All these different factors need to be taken into consideration when making realistic predictions on the population composition of L. monocytogenes and the conditions that can select for stress resistant variants. Altogether it can be concluded that the increased stress resistance as observed in the variants can be considered a trade-off. In the simulated model food chain, the WT clearly has a higher fitness, while the rpsU variant is more robust. On the other hand, there are combinations of mild stress conditions allowing for equal growth of WT and variant. Translation to actual food matrices and inclusion of more factors, like multiplicative effects of history on stress resistance and the effect of recovery conditions on stress survival, would be the next challenging step in making more realistic predictions on the effect of stress resistant variants on population composition in food products and processing environments. The current data illustrate the effect that environmental factors can have on population dynamics of L. monocytogenes and are a first step in evaluating the potential impact of population diversity on food safety. 


\section{References}

1. Ferreira, V., M. Wiedmann, P. Teixeira, and M.J. Stasiewicz, Listeria monocytogenes persistence in food-associated environments: Epidemiology, strain characteristics, and implications for public health. J ournal of Food Protection, 2014. 77(1): p. 150-170.

2. Dubnau, D. and R. Losick, Bistability in bacteria. Molecular Microbiology, 2006. 61(3): p. 564572.

3. Graumann, P.L., Different genetic programmes within identical bacteria under identical conditions: The phenomenon of bistability greatly modifies our view on bacterial populations. Molecular Microbiology, 2006. 61(3): p. 560-563.

4. Veening, J.W., W.K. Smits, and O.P. Kuipers, Bistability, epigenetics, and bet-hedging in bacteria. Annual Review of Microbiology, 2008. 62: p. 193-210.

5. Avery, S.V., Microbial cell individuality and the underlying sources of heterogeneity. Nature Reviews Microbiology, 2006. 4(8): p. 577-587.

6. Van Boeijen, I.K.H., R. Moezelaar, T. Abee, and M.H. Zwietering, Inactivation kinetics of three Listeria monocytogenes strains under high hydrostatic pressure. J ournal of Food Protection, 2008. 71(10): p. 2007-2013.

7. Karatzas, K.A.G. and M.H.J. Bennik, Characterization of a Listeria monocytogenes Scott A isolate with high tolerance towards high hydrostatic pressure. Applied and Environmental Microbiology, 2002. 68(7): p. 3183-3189.

8. Karatzas, K.A.G., A. Zervos, C.C. Tassou, C.G. Mallidis, and T.J . Humphrey, Piezotolerant small-colony variants with increased thermotolerance, antibiotic susceptibility, and lowinvasiveness in a clonal Staphylococcus aureus population. Applied and Environmental Microbiology, 2007. 73(6): p. 1873-1881.

9. Metselaar, K.I., H.M.W. Den Besten, T. Abee, R. Moezelaar, and M.H. Zwietering, Isolation and quantification of highly acid resistant variants of Listeria monocytogenes. International J ournal of Food Microbiology, 2013. 166(3): p. 508-514.

10. O'Driscoll, B., C.G.M. Gahan, and C. Hill, Adaptive acid tolerance response in Listeria monocytogenes: Isolation of an acid-tolerant mutant which demonstrates increased virulence. Applied and Environmental Microbiology, 1996. 62(5): p. 1693-1698.

11. Van Boeijen, I.K.H., C. Francke, R. Moezelaar, T. Abee, and M.H. Zwietering, Isolation of highly heat-resistant Listeria monocytogenes variants by use of a kinetic modeling-based sampling scheme. Applied and Environmental Microbiology, 2011. 77(8): p. 2617-2624.

12. Metselaar, K.I., H.M.W. den Besten, J . Boekhorst, S.A.F.T. van Hijum, M.H. Zwietering, and T. Abee, Diversity of acid stress resistant variants of Listeria monocytogenes and the potential role of ribosomal protein S21 encoded by rpsU. Frontiers in Microbiology, 2015. 6:422.

13. Van Boeijen, I.K.H., A.A.E. Chavaroche, W.B. Valderrama, R. Moezelaar, M.H. Zwietering, and T. Abee, Population diversity of Listeria monocytogenes LO28: Phenotypic and genotypic characterization of variants resistant to high hydrostatic pressure. Applied and Environmental Microbiology, 2010. 76(7): p. 2225-2233.

14. Maharjan, R., S. Nilsson, J . Sung, K. Haynes, R.E. Beardmore, L.D. Hurst, . . I. Gudelj, The form of a trade-off determines the response to competition. Ecology Letters, 2013. 16(10): p. 1267-1276.

15. Nyström, T., Growth versus maintenance: A trade-off dictated by RNA polymerase availability and sigma factor competition? Molecular Microbiology, 2004. 54(4): p. 855-862.

16. Phan, K. and T. Ferenci, A design-constraint trade-off underpins the diversity in ecologically important traits in species Escherichia coli. ISME J ournal, 2013. 7(10): p. 2034-2043.

17. Doyle, M.E., A.S. Mazzotta, T. Wang, D.W. Wiseman, and V.N. Scott, Heat resistance of Listeria monocytogenes. J ournal of Food Protection, 2001. 64(3): p. 410-429. 
18. Juneja, V.K., T.A. Foglia, and B.S. Marmer, Heat resistance and fatty acid composition of Listeria monocytogenes: Effect of $\mathrm{pH}$, acidulant, and growth temperature. Journal of Food Protection, 1998. 61(6): p. 683-687.

19. Metselaar, K.I., P. Saá Ibusquiza, A.R. Ortiz Camargo, M. Krieg, M.H. Zwietering, H.M.W. den Besten, and T. Abee, Performance of stress resistant variants of Listeria monocytogenes in mixed species biofilms with Lactobacillus plantarum. International J ournal of Food Microbiology, 2015. 213: p. 24-30.

20. Biesta-Peters, E.G., M.W. Reij, H. Joosten, L.G.M. Gorris, and M.H. Zwietering, Comparison of two optical-density-based methods and a plate count method for estimation of grouth parameters of Bacillus cereus. Applied and Environmental Microbiology, 2010. 76(5): p. 1399-1405.

21. Aryani, D.C., H.M.W. den Besten, W.C. Hazeleger, and M.H. Zwietering, Quantifying strain variability in modeling growth of Listeria monocytogenes. International J ournal of Food Microbiology, 2015. 208: p. 19-29.

22. Ratkowsky, D.A., J. Olley, T.A. McMeekin, and A. Ball, Relationship between temperature and growth rate of bacterial cultures. J ournal of Bacteriology, 1982. 149(1): p. 1-5.

23. Luong, J.H.T., Kinetics of ethanol inhibition in alcohol fermentation. Biotechnology and Bioengineering, 1985. 27(3): p. 280-285.

24. Zwietering, M.H., H.G.A.M. Cuppers, J.C. de Wit, and K. van't Riet, Evaluation of data transformations and validation of a model for the effect of temperature on bacterial growth. Applied and Environmental Microbiology, 1994. 60(1): p. 195-203.

25. Zwietering, M.H., T. Wijtzes, F.M. Rombouts, and K. van't Riet, A decision support system for prediction of microbial spoilage in foods. Journal of Industrial Microbiology, 1993. 12(3-5): p. 324-329.

26. Biesta-Peters, E.G., M.W. Reij, L.G.M. Gorris, and M.H. Zwietering, Comparing nonsynergistic gamma models with interaction models to predict growth of emetic Bacillus cereus when using combinations of $\mathrm{pH}$ and individual undissociated acids as growth-limiting factors. Applied and Environmental Microbiology, 2010. 76(17): p. 5791-5801.

27. Buchanan, R.L., R.C. Whiting, and W.C. Damert, When is simple good enough: A comparison of the Gompertz, Baranyi, and three-phase linear models for fitting bacterial growth curves. Food Microbiology, 1997. 14(4): p. 313-326.

28. Koutsoumanis, K., A. Stamatiou, P. Skandamis, and G.J.E. Nychas, Development of a microbial model for the combined effect of temperature and $\mathrm{pH}$ on spoilage of ground meat, and validation of the model under dynamic temperature conditions. Applied and Environmental Microbiology, 2006. 72(1): p. 124-134.

29. Geeraerd, A.H., V. Valdramidis, and J.F. Van Impe, GInaFiT, a freeware tool to assess nonlog-linear microbial survivor curves. International J ournal of Food Microbiology, 2005. 102(1): p. 95-105.

30. Den Besten, H.M.W., M. Mataragas, R. Moezelaar, T. Abee, and M.H. Zwietering, Quantification of the effects of salt stress and physiological state on thermotolerance of Bacillus cereus ATCC 10987 and ATCC 14579. Applied and Environmental Microbiology, 2006. 72(9): p. 5884-5894.

31. Ross, T., Indices for performance evaluation of predictive models in food microbiology. J ournal of Applied Bacteriology. 81(5): p. 501-8.

32. Zilelidou, E.A., V. Tsourou, S. Poimenidou, A. Loukou, and P.N. Skandamis, Modeling transfer of Escherichia coli O157:H7 and Listeria monocytogenes during preparation of fresh-cut salads: Impact of cutting and shredding practices. Food Microbiology, 2015. 45, Part B: p. 254-265.

33. Skandamis, P.N. and G.J.E. Nychas, Development and evaluation of a model predicting the survival of Escherichia coli O157:H7 NCTC 12900 in homemade eggplant salad at various temperatures, pHs, and oregano essential oil concentrations. Applied and Environmental Microbiology, 2000. 66(4): p. 1646-1653. 
34. Wang, W., M. Li, W. Fang, A.K. Pradhan, and Y. Li, A predictive model for assessment of decontamination effects of lactic acid and chitosan used in combination on Vibrio parahaemolyticus in shrimps. International J ournal of Food Microbiology, 2013. 167(2): p. 124-130.

35. Pan, Y. and F. Breidt Jr, Enumeration of viable Listeria monocytogenes cells by real-time PCR with propidium monoazide and ethidium monoazide in the presence of dead cells. Applied and Environmental Microbiology, 2007. 73(24): p. 8028-8031.

36. Abee, T., J. Koomen, K.I. Metselaar, M.H. Zwietering, and H.M. den Besten, Impact of pathogen population heterogeneity and stress-resistant variants on food safety. Annual Review in Food Science and Technology, 2016. 7:439-456.

37. Zwietering, M.H., J.C. de Wit, and S. Notermans, Application-of predictive microbiology to estimate the number of Bacillus cereus in pasteurised milk at the point of consumption. International J ournal of Food Microbiology, 1996. 30(1-2): p. 55-70.

38. Te Giffel, M.C. and M.H. Zwietering, Validation of predictive models describing the growth of Listeria monocytogenes. International J ournal of Food Microbiology, 1999. 46(2): p. 135-149.

39. Augustin, J.C. and V. Carlier, Modelling the growth rate of Listeria monocytogenes with a multiplicative type model including interactions between environmental factors. International J ournal of Food Microbiology, 2000. 56(1): p. 53-70.

40. Biesta-Peters, E.G., M.W. Reij, M.H. Zwietering, and L.G.M. Gorris, Comparing nonsynergy gamma models and interaction models to predict growth of emetic Bacillus cereus for combinations of $\mathrm{pH}$ and water activity values. Applied and Environmental Microbiology, 2011. 77(16): p. 5707-5715.

41. Le Marc, Y., V. Huchet, C.M. Bourgeois, J.P. Guyonnet, P. Mafart, and D. Thuault, Modelling the growth kinetics of Listeria as a function of temperature, $\mathrm{pH}$ and organic acid concentration. International J ournal of Food Microbiology, 2002. 73(2-3): p. 219-237.

42. Dalgaard, P., Fresh and lightly preserved seafoods, in Shelf-life evaluation of food. 2000, Aspen Publishing. p. 110-139.

43. Álvarez-Ordóñez, A., A. Fernández, A. Bernardo, and M. López, Heat-resistance prediction of Listeria innocua grown at different temperatures. J ournal of Food Safety, 2009. 29(3): p. 474-483.

44. Miller, F.A., B. Ramos, M.M. Gil, T.R.S. Brandão, P. Teixeira, and C.L.M. Silva, Influence of $\mathrm{pH}$, type of acid and recovery media on the thermal inactivation of Listeria innocua. International J ournal of Food Microbiology, 2009. 133(1-2): p. 121-128.

45. Jørgensen, F., T.B. Hansen, and S. Knøchel, Heat shock-induced thermotolerance in Listeria monocytogenes 13-249 is dependent on growth phase, $\mathrm{pH}$ and lactic acid. Food Microbiology, 1999. 16(2): p. 185-194.

46. Aryani, D.C., H.M.W. den Besten, W.C. Hazeleger, and M.H. Zwietering, Quantifying variability on thermal resistance of Listeria monocytogenes. International Journal of Food Microbiology, 2015. 193(0): p. 130-138.

47. Ivy, R.A., M. Wiedmann, and K.J. Boor, Listeria monocytogenes grown at $7^{\circ} \mathrm{C}$ shows reduced acid survival and an altered transcriptional response to acid shock compared to L. monocytogenes grown at $37^{\circ} \mathrm{C}$. Applied and Environmental Microbiology, 2012. 78(11): p. 3824-3836.

48. Guillier, L., P. Pardon, and J.-C. Augustin, Influence of stress on individual lag time distributions of Listeria monocytogenes. Applied and Environmental Microbiology, 2005. 71(6): p. 2940-2948.

49. Muñoz, M., L. Guevara, A. Palop, and P.S. Fernández, Prediction of time to grouth of Listeria monocytogenes using Monte Carlo simulation or regression analysis, influenced by sublethal heat and recovery conditions. Food Microbiology, 2010. 27(4): p. 468-475.

50. Aguirre, J.S., A. González, N. Özçelik, M.R. Rodríguez, and G.D. García de Fernando, Modeling the Listeria innocua micropopulation lag phase and its variability. International J ournal of Food Microbiology, 2013. 164(1): p. 60-69. 
51. Leguérinel, I., I. Spegagne, O. Couvert, L. Coroller, and P. Mafart, Quantifying the effects of heating temperature, and combined effects of heating medium $\mathrm{pH}$ and recovery medium $\mathrm{pH}$ on the heat resistance of Salmonella typhimurium. International J ournal of Food Microbiology, 2007. 116(1): p. 88-95.

52. Casadei, M.A., R.E. De Matos, S.T. Harrison, and J.E. Gaze, Heat resistance of Listeria monocytogenesin dairy products as affected by the growth medium.J ournal of Applied Microbiology, 1998. 84(2): p. 234-239. 


\section{Supplemental data}
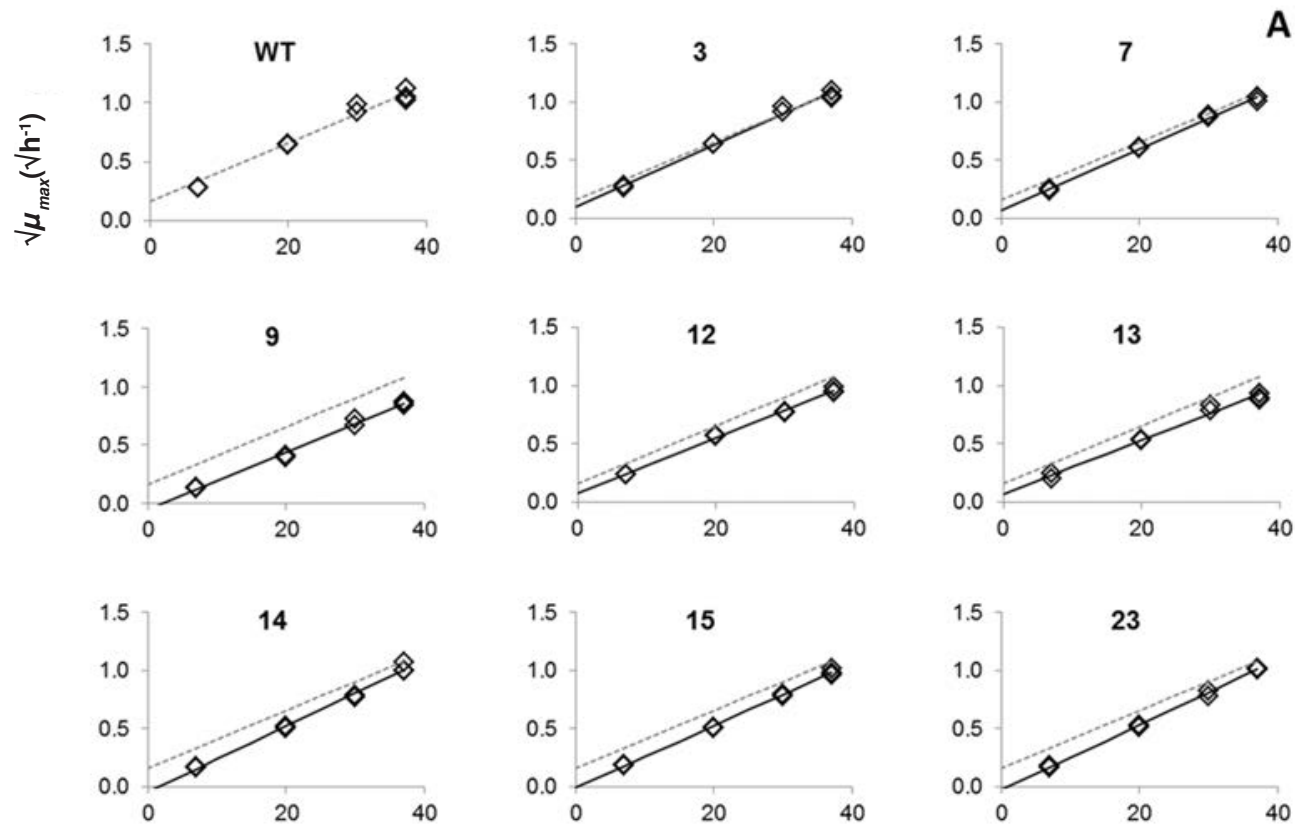

Temperature $\left({ }^{\circ} \mathrm{C}\right)$
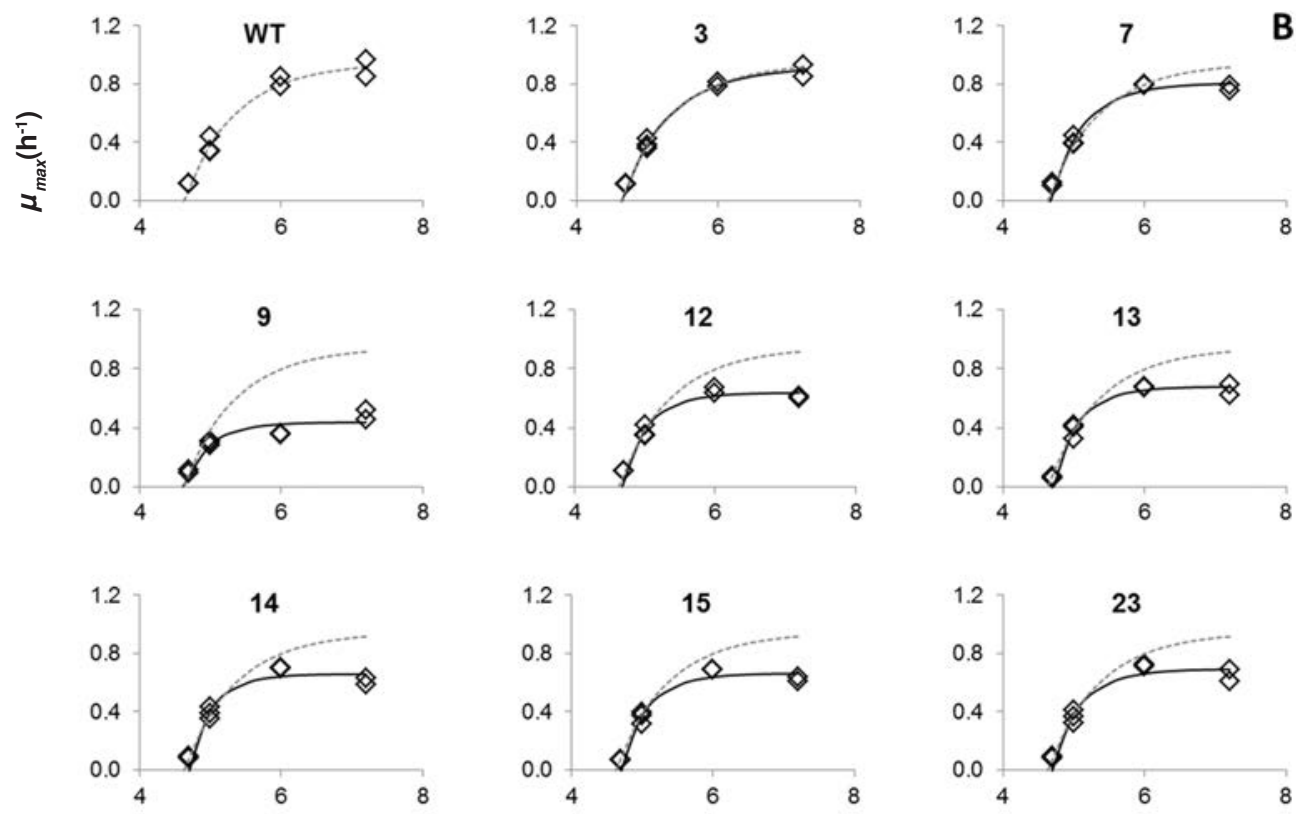

$\mathrm{pH}$ 

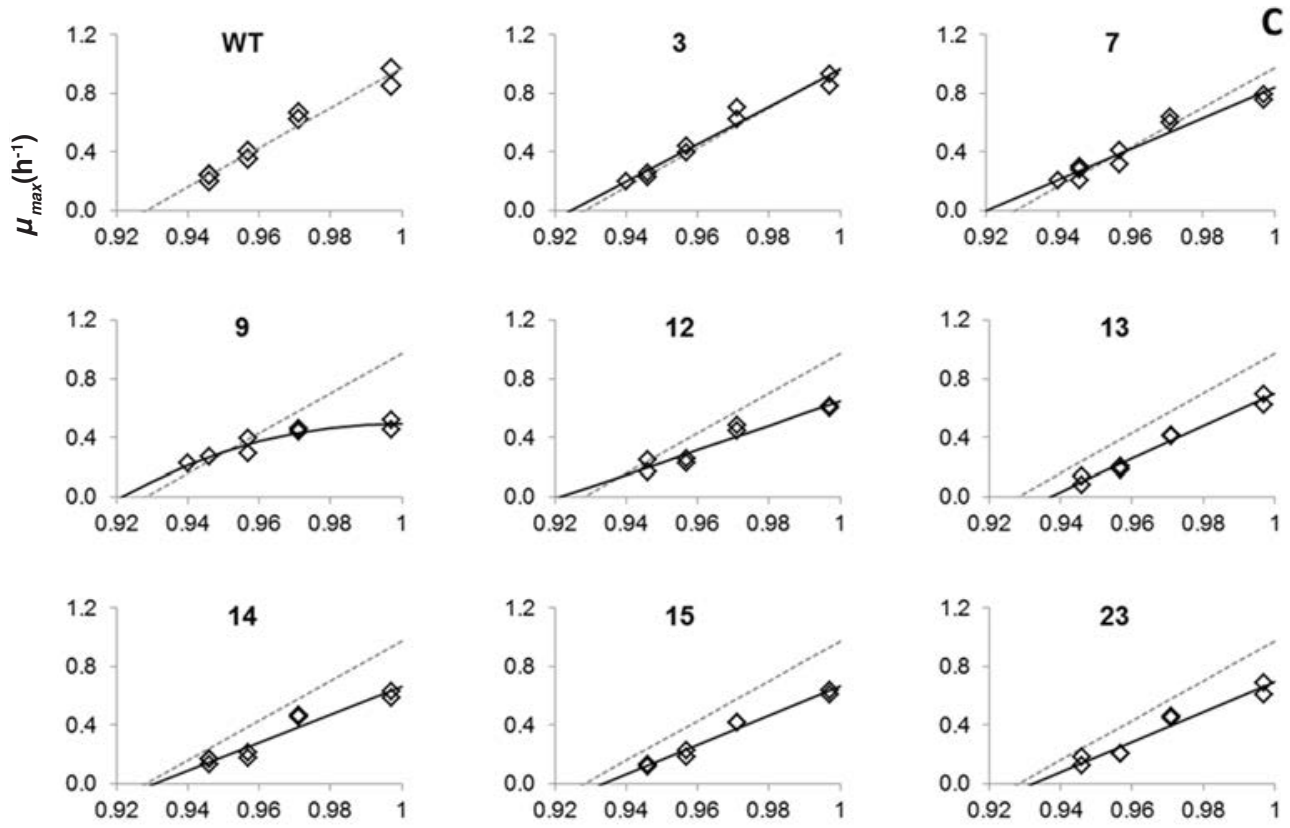

\section{$a_{w}$}

Figure S5.1: Maximum specific growth rate $\left(\mathrm{h}^{-1}\right)$ of $\mathrm{WT}$ and eight acid resistant variants under different temperature, $\mathrm{pH}$ and $\mathrm{a}_{\mathrm{w}}$ conditions in $\mathrm{BHI}$ (reference condition: $30^{\circ} \mathrm{C}, \mathrm{pH} 7.3, \mathrm{a}_{\mathrm{w}}$ 0.997). Cardinal growth parameters were estimated with the secondary growth models in equation 1-3 (--- WT, - variants). 

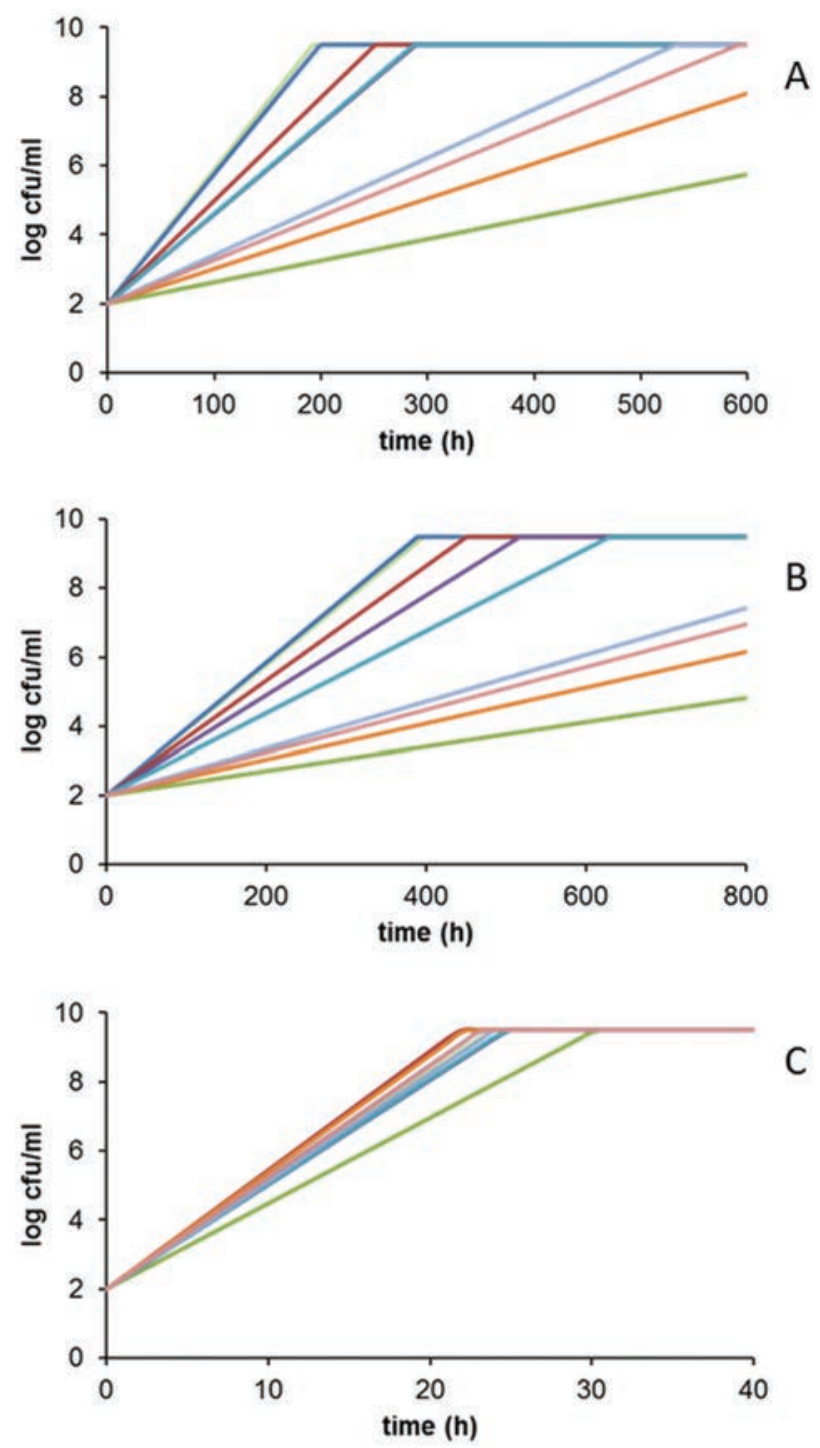

Figure S5.2: Growth predictions of L. monocytogenes LO28 WT and eight acid resistant variants according to the three-phase linear model. Growth predictions were based on cardinal growth parameters as shown in Table 1 and the gamma model (Equation 4). Three scenarios were considered: milk (pH 6.6; $\left.\mathrm{a}_{\mathrm{w}} 0.997 ; 7^{\circ} \mathrm{C}\right)(\mathrm{A})$, ham (pH 6.0; $\left.\mathrm{a}_{\mathrm{w}} 0.965 ; 7^{\circ} \mathrm{C}\right)(\mathrm{B})$ and $\mathrm{BHI}$ at $\mathrm{pH} 5.2$ at $37^{\circ} \mathrm{C}(\mathrm{C})$.

$-\mathrm{WT}-3-7-9-12-13-14-15-23$. 


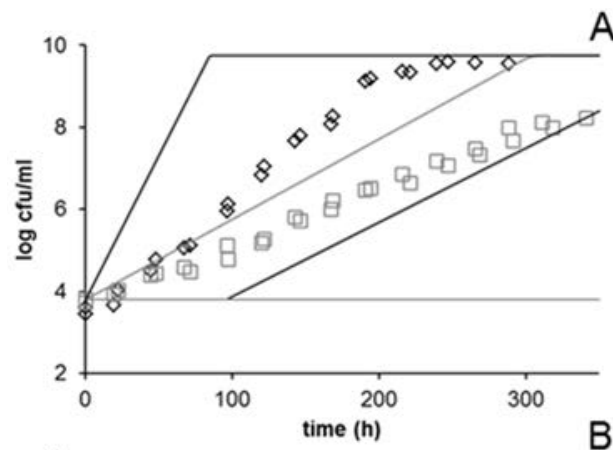

A1

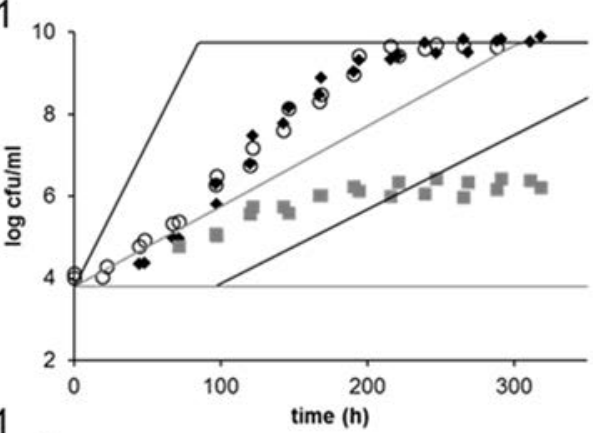

$\mathrm{A} 2$
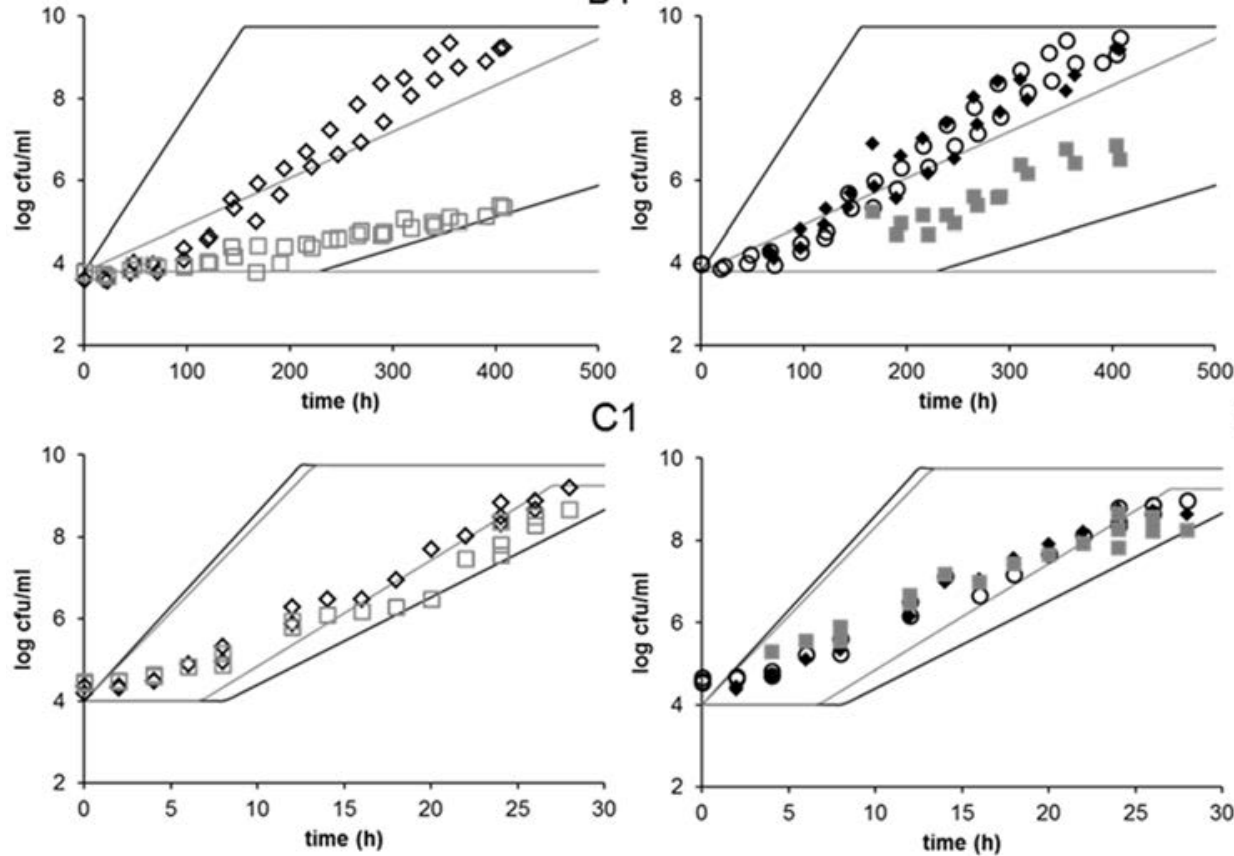

Figure S5.3: 95\% prediction interval and validations for L. monocytogenes LO28 WT (black, counts diamonds) and acid resistant variant 14 (grey, counts squares). Growth predictions according to the three-phase linear model were based on upper and lower 95\% confidence interval of cardinal growth parameters as shown in Table 5.1 and the gamma model. 3 scenarios were considered: milk (BHI-M, pH 6.6; $\left.\mathrm{a}_{\mathrm{w}} 0.997 ; 7^{\circ} \mathrm{C}\right)(\mathrm{A})$, ham (BHI-B, pH 6.0; $\left.\mathrm{a}_{\mathrm{w}} 0.965 ; 7^{\circ} \mathrm{C}\right)(\mathrm{B})$ and $\mathrm{BHI}$ at pH 5.2 at $37^{\circ} \mathrm{C}(\mathrm{BHI}-\mathrm{S})(\mathrm{C})$.For each scenario two situations were considered, namely no lag phase (upper line) and a maximum lag phase of $\mu \cdot \lambda=4$ (lower line). Validations were done by plate counts in single cultures (1) and mixed cultures (2). For the mixed cultures, total plate counts (circles) are displayed by the open symbols and individual contributions of WT and variant 14 in the mixture were deducted from $\mathrm{c}_{\mathrm{t}}$ values and the standard curves correlating $\mathrm{c}_{\mathrm{t}}$ values to $\log \mathrm{cfu} / \mathrm{ml}$ counts (closed symbols). 

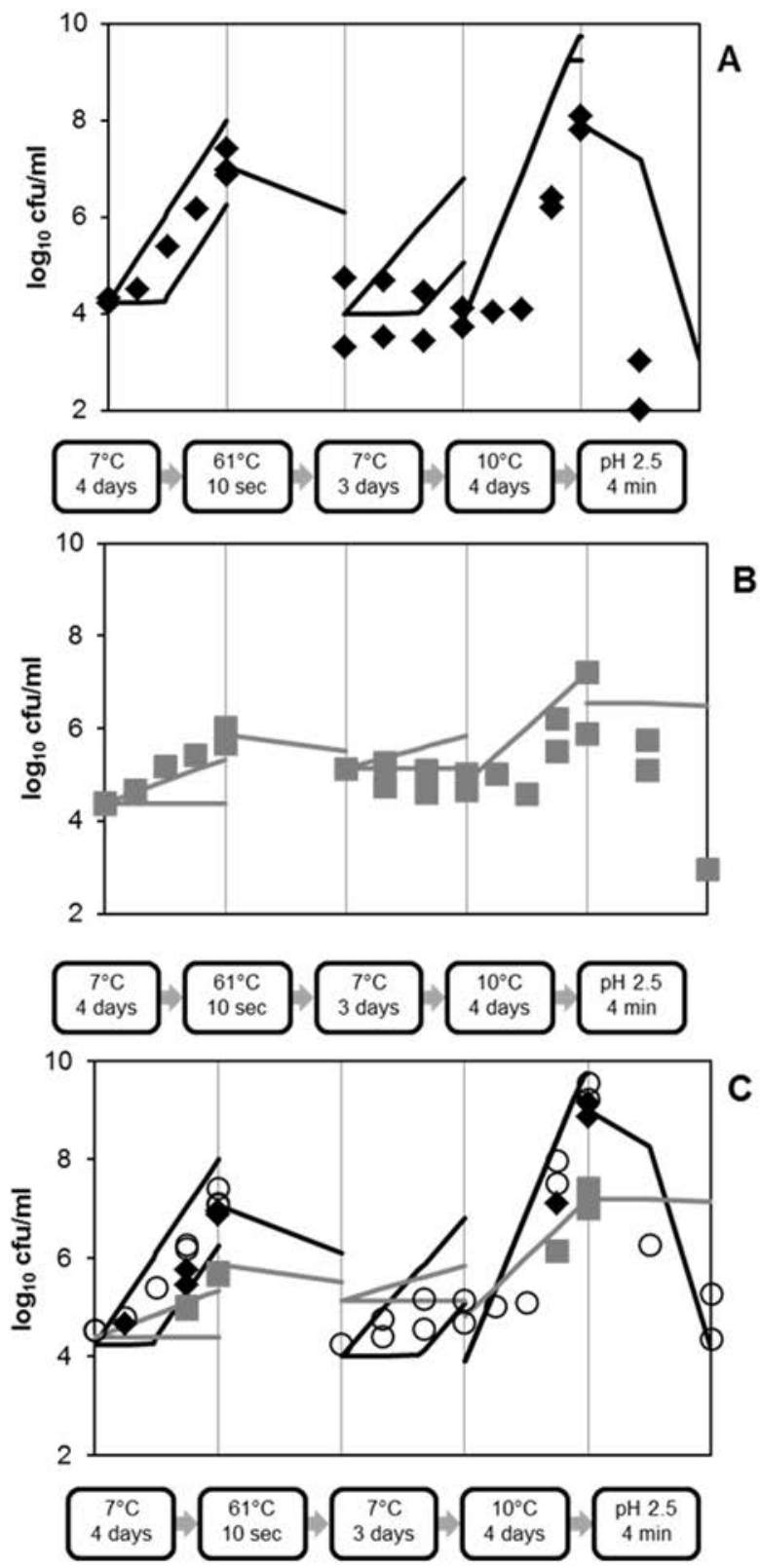

Figure S5.4: Performance of WT (black) and variant 14 (grey) along a model food chain. The chain was simulated in BHI-M (pH 6.6). Growth and inactivation behavior was predicted (solid lines) based on growth and inactivation kinetics as shown in Table 5.1-5.3 and Figure 5.1, 5.3 and 5.4. Predictions were validated in $\mathrm{BHI}$ set to $\mathrm{pH} 6.6$ by $10 \mathrm{M} \mathrm{HCl}$, simulating milk characteristics. Two scenarios were considered for the growth steps, namely no lag time (upper line) and a maximum lag time of $\mu \cdot \lambda=4$ (lower line). Validations were done in single cultures (A: WT, B: variant 14) and in mixed cultures (C). Open symbols represent the plate counts and closed symbols the individual contributions of WT (black) and variant 14 (grey) in the mixture as deducted from $c_{t}$ values and the standard curves correlating $c_{t}$ values to $\log \mathrm{cfu} / \mathrm{ml}$ counts. 


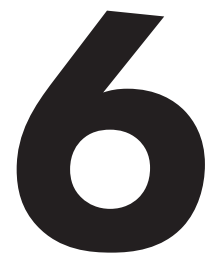

General discussion, conclusion and future perspectives 
Listeria monocytogenes is a robust pathogen and well equipped to adapt to different environmental niches. Its robustness and adaptive behaviour make it a difficult pathogen to eliminate from food processing environments. L. monocytogenes contamination of food products has resulted in numerous listeriosis outbreaks, often with severe consequences [1-5]. The public health as well as the economic burden of listeriosis outbreaks are high and therefore a lot of research in the last decades has focussed on better understanding of the behaviour of this pathogen, which ultimately has to lead to better control measures. One of the potential challenges in L. monocytogenes control is the presence of stress resistant subpopulations. The presence of such subpopulations can lead to tailing of inactivation curves upon stress exposure, which can be a major concern in food industry, especially with the recent trend towards minimally processed foods. In previous research, inactivation of $\mathrm{L}$. monocytogenes by high hydrostatic pressure (HHP) and heat revealed considerable tailing of inactivation curves and stable resistant variants were isolated from the tail $[6,7]$. Phenotyping and genomic analysis of L. monocytogenes LO28 variants revealed great population diversity, including a subpopulation typified by mutations and deletions in the ctsR gene. Although alteration of ctsR has been shown to contribute largely to the occurrence of stress resistant variants of L. monocytogenes, this is thought to be only one of the mutations underlying population heterogeneity, and additional genetic variations which lead to increased stress tolerance remain to be elucidated. The objective of this research was to extend the knowledge on the generation and occurrence of stress resistant variants in populations of L. monocytogenes and the mechanisms leading to increased resistance, and to investigate the potential impact of the occurrence of these mutations and corresponding phenotypes on population dynamics and food safety. The first step in this research was to extend the available set of variants obtained after exposure to heat and HHP with a set of variants isolated after exposure to acid stress. Also acid stress was shown to lead to tailing of inactivation curves and to selection for stable stress resistant variants. These acid resistant variants showed both unique and overlapping features when comparing them to the previously isolated stress resistant variants obtained from heat and HHP treated cultures. Novel information obtained in this study includes mechanistic, qualitative and quantitative knowledge on L. monocytogenes population heterogeneity which are discussed in more detail in this chapter together with the potential impact on food safety. 


\section{Mechanisms of increased resistance}

\section{Genetic background of stress resistant variants}

At the start of this research, the only identified mutation in stable resistant L. monocytogenes variants was a mutation in the class III heat shock repressor ctsR [6, 8-11]. Both heat and HHP exposure were shown to lead to selection for, amongst others, these ctsR variants. The ctsR variants showed a multiple stress resistant phenotype and a reduced growth rate, which was linked to the increased expression of genes encoding Clp proteases, as a result of the defect in repressor function of CtsR [11]. Also, the ctsR variants were shown to have a reduced virulence potential in a mouse model [12]. The set of stable resistant variants isolated after exposure to HHP and heat was extended by variants isolated after acid stress (Chapter 2). Acid stress was chosen as it is an important hurdle both in food preservation, as well as in stomach passage [13]. In Chapter 2 it was shown that indeed also acid stress leads to selection for stable stress resistant variants. This was an important finding as it indicated that multiple types of stress can select for stable stress resistant variants. The newly isolated acid resistant variants were also resistant to heat and showed reduced growth rates, but none of the 23 variants had a mutation in ctsR or upstream region. This indicated that, despite similarities with the phenotype caused by a ctsR alteration, other mutations are responsible for the observed phenotypes of the acid isolated variants. Whole genome sequencing, followed by SNP analysis revealed a mutation in rpsU or its upstream region in a large subset of variants. This gene, encoding ribosomal protein S21, does not have the obvious link to increased stress resistance such as the ctsR mutation has, but the strong correlation between the phenotypic cluster of 11 variants and the mutation in the rpsU region of the same 11 variants indicated that reduction or loss of production of this ribosomal protein is linked to the increased resistance of these variants. In Chapter 3, the potential role of rpsU mutations on stress resistance was discussed. One of the possibilities is that the reduced growth rate of the rpsU variants plays a rolein theincreased stress resistance [14]. From Chapter 2 and 4 it is clear that also in stationary phase, when the cells are not growing anymore, the rpsU variants show increased resistance, indicating that if growth rate plays a role in the increased resistance of the variants, this is not the sole explanation for the observed phenotype. Another possibility discussed in Chapter 3 is linked to the increased GAD activity which was observed in the rpsU variants and some of the other variants as well. It was suggested that the increased GAD activity was associated with higher levels of intracellular glutamate supporting increased conversion from glutamate to GABA and thereby consuming more intracellular protons resulting in better homeostasis of the internal $\mathrm{pH}$ [15]. Increased levels of 
intracellular glutamate would direct to changes in metabolism [16], which is a likely scenario, given the phenotypes we observed. On the other hand, the environmental conditions also play an important role in the stress resistance of the variants, as was demonstrated in Chapters 4 and 5. It was shown that the rpsU variants show highly increased acid resistance when grown under optimal conditions, but when grown at lower temperature and/ or in a low $\mathrm{pH}$ environment, they showed only little to no increased resistance compared to the WT. Other variants, with unknown mutations, were less affected by the environmental conditions. The fact that the environmental conditions play an important role in the stress resistance of the rspU variants supports the hypothesis that the increased resistance is caused by a metabolic shift, which is highly affected by environmental conditions. A microarray analysis was performed to get more insight in the mechanisms underlying the role of ribosomal proteins in the increased stress resistance. In late-exponential phase cultures grown in $\mathrm{BHI}$ at $30^{\circ} \mathrm{C}$, around 500 genes were significantly up- or downregulated in two rpsU variants (variant 14 and 15) compared to the WT, of which about half were upregulated and half downregulated. In Figure 6.1 the distribution of the clusters of orthologous groups (COGs) that are up- or downregulated in rpsU variant 14 are shown. Notably, a similar pattern was observed for variant 15.

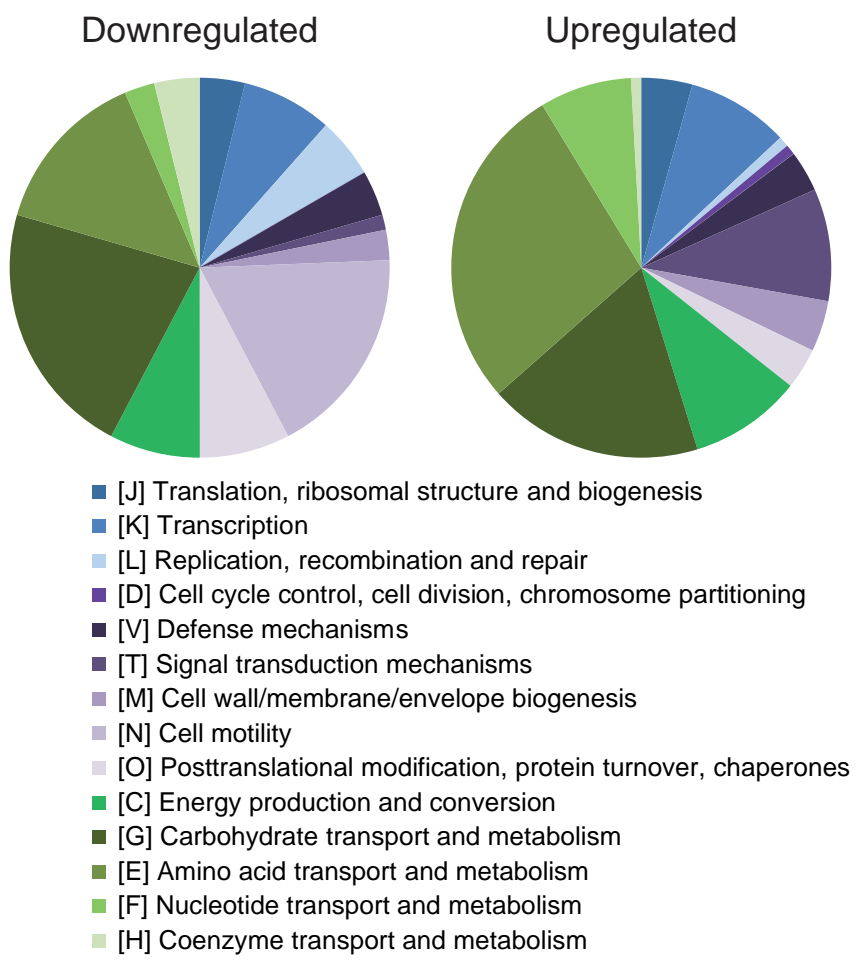

Figure 6.1: Up- and downregulated COG categories in rpsU variant 14 in late-exponential phase in BHI at $30^{\circ} \mathrm{C}$ compared to the WT. In total 203 genes were significantly downregulated and 236 significantly upregulated. 
An initial analysis of the transcriptome data showed clearly that carbohydrate metabolism and amino acid metabolism are affected in the rpsU variants, supporting the hypothesis that the increased stress resistance of the rpsU variants is linked to metabolic changes. Another interesting observation is the downregulation of all the genes involved in cell motility. This was somehow surprising as all the rpsU variants were shown to be motile in Chapter 3. On the other hand, the conditions in the assay used in Chapter 3 were different from the late-exponential phase liquid cultures that were used for the transcriptome analysis. Also, the reduced expression of motilityrelated genes is in line with observations in a $\Delta \mathrm{rps} U$ mutant of Bacillus subtilis which showed to be immotile [17], indicating a role of rpsU in bacterial motility. Further analysis of the transcriptome data and pathway analysis combined with metabolite analysis will give more insights in the mechanisms of increased resistance.

\section{Presence of variants in the population}

It was surprising that none of the acid isolated variants had a ctsR mutation, as quite some overlapping features between HHP and acid isolated variants were observed. Amongst the variants isolated after both types of stress, a large fraction showed a multiple stress resistant phenotype (heat, acid, benzalkonium chloride), a reduced growth rate and smaller colonies. Since the mutation underlying the increased resistance of the majority of the HHP and heat selected variants was not known yet, these variants were evaluated on the presence of rpsU mutations as well. In Figure 6.2 it can be seen that the rpsU mutation was predominantly present in the acid isolated variants, but they were also found in two HHP isolated variants and in one variant isolated after heat exposure.

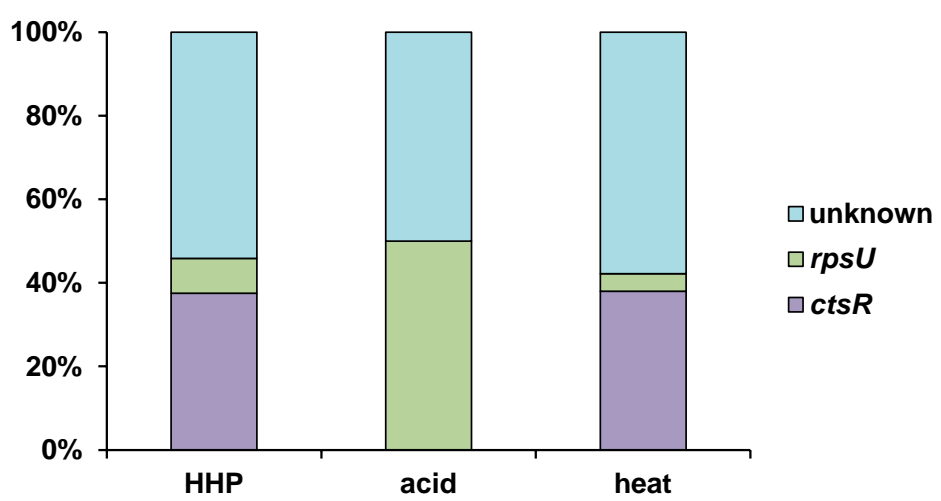

Figure 6.2: Overview of ctsR and rpsU mutations in L. monocytogenes $\mathrm{LO} 28$ stress resistant variants, isolated after HHP $(n=24)$, acid $(n=23)$ and heat $(n=24)$ exposure. The conceivable mutations in variants with intact rpsU and ctsR genes are classified as 'unknown'. 
The fact that the rpsU variants were also found after heat and HHP stress suggests that these variants are widely present in the population and that different types of stress may lead to selection for rpsU variants. Notably, in Chapter 5 (Figure 5.5) it was calculated that the probability of isolating an rpsU variant after $8 \mathrm{~min}$ at $55^{\circ} \mathrm{C}$ is around $1 \%$, which corresponds with the low number of rpsU variants amongst the variants described by Van Boeijen at al. [6] isolated after heat exposure. The data in Figure 6.2 and the calculations of the probability to find variants after certain stress exposures presented in Chapter 5 suggest that different types of stress can lead to selection for the same type of variant and also that the same type of stress leads to selection for different types of variants. It remains a pivotal question whether these variants are present in the population or if the mutations are induced by the stress exposure. In every growing bacterial culture, mutations are introduced with a spontaneous mutation rate of around 0.0033 mutations per genome [18]. Some of these mutations will not have an effect on the robustness and/or fitness of the bacterial cell, simply because they are in non-coding regions of the genome, because they do not result in an amino acid change or because they do not affect protein function. Some mutations might be deleterious and by the rules of evolution these will be generally outcompeted by the majority of the population showing a higher degree of fitness $[19,20]$. In the case of the stress resistant variants, the introduced mutations can be advantageous for the population and prepares the population for less favourable conditions. However, these types of mutations are generally a tradeoff and it depends on the environmental conditions if a mutation poses an advantage or a disadvantage [21]. One common characteristic of a significant number of the rpsU and ctsR mutations is that they are present in a region with a high number of short tandem repeats. Many of the ctsR mutations are in the three glycine (GGT) repeats and the rpsU mutations in the ribosome binding site (RBS) with many A and $\mathrm{G}$ repeats (Chapter 3). Various studies indicate that regions with short tandem repeats show increased rates of spontaneous mutations through strand slippage [22] and that tandem repeats are very common in stress response genes [23]. It is possible that this mechanism of strategically located hyper mutable regions for the generation of population variability is widespread among several species of bacteria [24]. However, not all of the rpsU and ctsR mutations were located in the sequences with many repeats, suggesting that the mutations could also be a random event. Van Boeijen et al. [6] calculated the chance of random mutations in the ctsR gene and this value was very close to the ctsR fraction that was calculated based on the inactivation kinetics of the WT and ctsR variant. The chance of rpsU mutations is slightly lower due to its smaller gene size, which also corresponds to a slightly lower rpsU fraction than ctsR fraction in the LO28 WT population. It is also well known that stress can induce mutations and genetic variation which is often referred to as adaptive mutagenesis [25]. This process of adaptive mutagenesis has been defined 
as a process that produces advantageous mutations during exposure to non-lethal stress. Non-lethal stress is also frequently encountered in food products and during food processing. The SOS response is thought to play an important role in this adaptive mutagenesis in L. monocytogenes [26]. The SOS response is activated upon stress exposure and thereby a translesion DNA polymerase with a low specificity is activated. This polymerase is prone to introducing errors during DNA damage repair, generally resulting in point mutations [27]. This adaptive mutagenesis introduces both beneficial and deleterious mutations at the same time, but is thought to increase the overall fitness of the population during stress by generating genetic variation [19, 27]. Although random mutations can occur during growth under optimal conditions, adaptive mutagenesis is not very likely to happen during growth to late-exponential phase, since no significant stress is imposed on the cells and thus no trigger to activate the SOS response. It cannot be excluded that the relatively short lethal acid exposures used in our research to isolate the variants causes DNA damage and subsequent activation of the SOS response. Altogether, taken into account the abovementioned random mutation rates that correspond to the variant fractions, it is conceivable that the mutations found in the variants result from random mutations introduced during growth that are selected for during stress exposure. It is apparent that next to rpsU and ctsR mutations, other mutations, which are yet to be elucidated, can also pose an advantage for L. monocytogenes subpopulations under specific conditions.

\section{Quantifying population heterogeneity}

\section{Non-linear inactivation}

The work described in this research, as well as from previously published research $[6,7]$, clearly show that non-linear inactivation kinetics are often observed upon stress exposure. No matter the underlying mechanisms of the observed tailing, for risk assessors and food producers using predictive microbiology to estimate the effect of a certain processing step on the microbial stability of a food product this non-linear behaviour is of importance and should be taken into consideration. Neglecting the tail can lead to serious overestimation of processing efficiency when the tailing effect is bigger than the biological error, and can cause serious problems in minimal processing with a narrow margin of safety. It is therefore important that accurate models are available to describe non-linear behaviour providing reliable estimates of the different model parameters. The model found to best describe biphasic behaviour with a shoulder period and which has parameters with a biological interpretation is the model of Geeraerd et al. [28]. One drawback of this model was that the parameter f, i.e. the resistant fraction, was difficult to estimate, as 
it gave a wide confidence interval in many cases. Even in cases where visually there was clearly a significant two-phase behaviour, the parameter $f$ was found to be not significant. Reparameterization of the model is described in Chapter 2 and resulted in increased performance of the model and mainly better statistical estimation of the resistant fraction parameter. Model reparameterization is often done when a certain parameter is not in a similar order of magnitude as the other parameters [29]. The reparameterization of the biphasic model is a significant step in better estimation of the resistant fraction within bacterial populations.

\section{Resistant fraction and population composition}

From Chapter 5 it is clear that a good estimation of the resistant fraction is very valuable when estimating the probability of isolating stable resistant variants upon different environmental conditions and when evaluating how different environmental conditions can affect the population composition. The kinetic modelling based sampling scheme method by Van Boeijen et al. [6] used in Chapter 5 highly depends on a good estimate of the initial fraction of variants in the population. Also, themethod is sensitive to small deviations in the kinetics, which is illustrated in Figure 6.3.

Theinactivation kinetics at $\mathrm{pH} 2.5$ for two rpsUvariants (variant 14 and 15) are rather similar, but in the case of variant 14 the biphasic model with shoulder gave the best statistical fit, and in the case of variant 15 the linear model gave the best statistical fit, as evaluated by the F-test and lack-of-fit test. From the data points in Figure 6.3 it is clear that this difference in best fitting model is mostly due to one outlier of variant 15 at $20 \mathrm{~min}$, and the otherwise limited number of data points at the end of the curve. When these kinetic parameters are used to estimate the fraction of rpsU variants within the WT population, two different patterns are observed. Although both of them have a steep peak up after 5 min (which coincides with the transition from sensitive to resistant fraction in the WT) the order of magnitude is $\sim 10$-fold different and the probability curve has a different shape. Figure 6.3 illustrates that specific stress and exposure times exist allowing for more efficient selection of stable resistant variants. However, care should be taken in the interpretation and factors like biological and experimental variability should be taken into account. Large variability is often observed upon stress exposure and therefore the probability of selecting for certain variants may change from one experiment to the other. This is also illustrated by the observation that the probability of finding an rpsU variant after exposure to $55^{\circ} \mathrm{C}$ for $25 \mathrm{~min}$ is around 9\% according to Figure 5.5, whereas Van Boeijen et al. [6] found no stable heat resistant variants under the same conditions. This is most likely due to the different method of heat inactivation and the different initial level of cells, which highlights the difficulty in predicting the probability that stable resistant variants will be selected for. The knowledge on the fraction of 

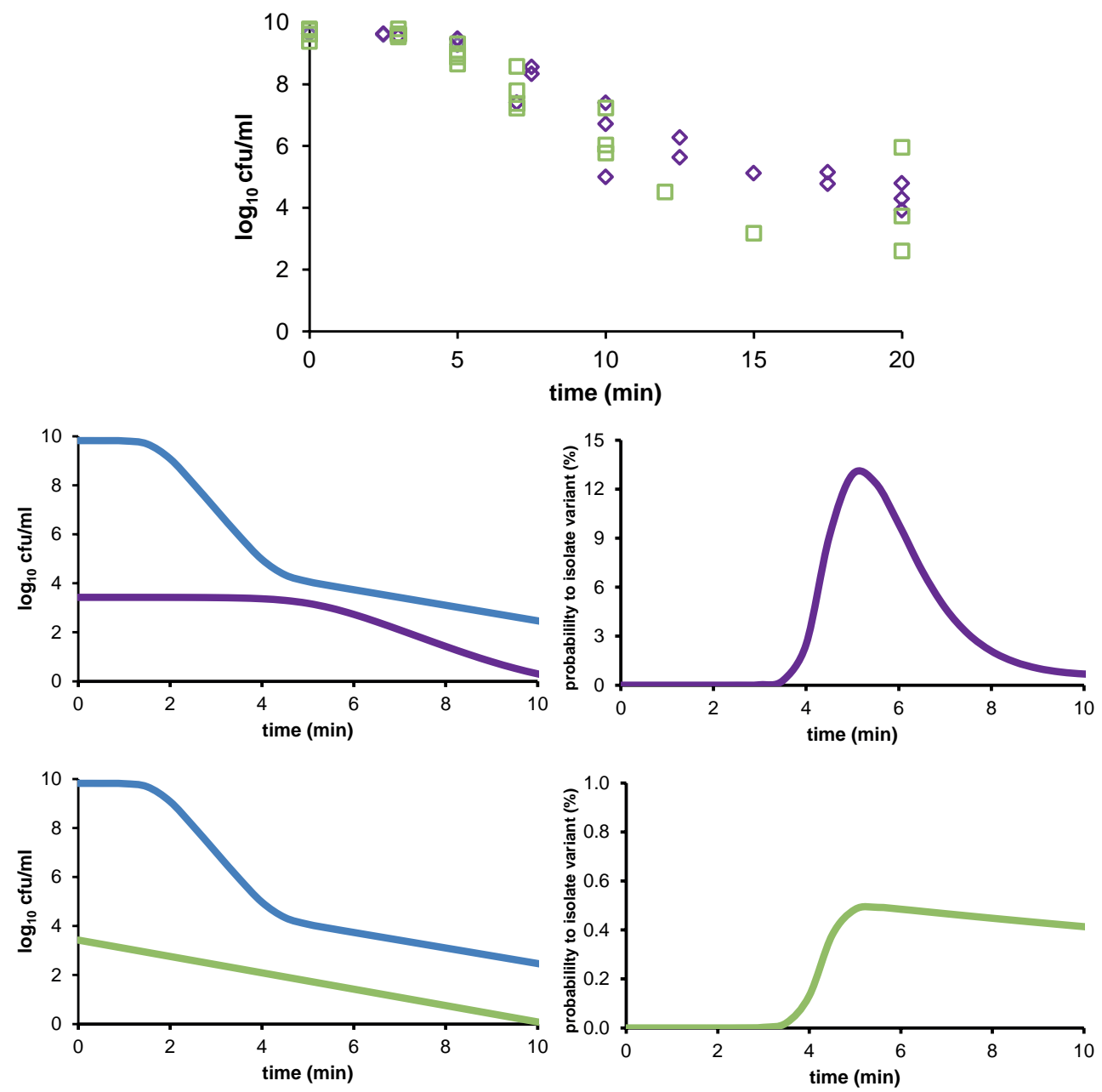

Figure 6.3: Probability calculations affected by inactivation kinetics and resistant fraction estimate. The upper panel shows the inactivation kinetics of variant 14 (purple, diamonds) and variant 15 (green, squares) upon exposure to $\mathrm{pH}$ 2.5. The left panels show the model fit of both variants and the WT (blue) considering the rpsU fraction to be $5 \cdot 10^{-7}$. The right panel shows the probability of finding an rpsU variant based on the respective inactivation kinetics.

variants with the same genetic background or within the same phenotypic cluster within the tail (the total resistant fraction) of the inactivation curve is very valuable for making predictions on the population dynamics under industrially relevant conditions. From the predictions and validations in Chapter 5, it is clear that not only the variability should be taken into account when making predictions on the fraction of variants in a population. Also the history and recovery conditions have a large impact on the growth and inactivation kinetics and thus on the probability that variants are selected for. 


\section{Heterogeneity as variability factor}

Knowledge on variability factors is needed to make realistic estimates of growth and inactivation parameters which can be used in exposure assessments or models for shelf-life prediction. Many researchers have evaluated and/or quantified different variability factors for several microorganisms [30-36]. Population heterogeneity and the presence of variants can be considered a variability factor as well, and quantification of non-linear inactivation behaviour is therefore of relevance in predictive microbiology. Deterministic models, as used in this study, provide a mean estimate with a prediction interval of the reduction in microbial load achieved by a certain processing step. Naturally, food processors will rather stay on the safe side of this prediction but also do not wish to over-process their products. The impact of variability factors on model predictions of L. monocytogenes has been quantified by Aryani et al. [30, 31]. The authors quantified and compared the effect of strain variability, biological variability and experimental variability on growth and inactivation kinetics of L. monocytogenes. Also the effect of growth history on stress resistance was evaluated. A series of 20 different strains were included in both studies and this provided a range of $\mathrm{D}$-values and cardinal growth parameters and the corresponding variability factors, which allowed for prioritization of the different variability factors. Growth and inactivation parameters were also benchmarked with an extensive set of literature data on cardinal growth parameters [37-39] and D-values [40] of L. monocytogenes. This data collection extracted from literature includes many different variability factors since the data was obtained from different studies, using different strains and different experimental conditions. When the upper limit in the range of D-values or growth rates is used, the predictions are conservative but can be assumed to be realistic. Quantification of different variability factors is of importance to evaluate which variability factors are most important to be taken into account when making predictions. To compare population heterogeneity to other variability factors, the growth and inactivation parameters obtained in Chapter 5 were included in the range of the literature values and the $20 \mathrm{~L}$. monocytogenes strains used by Aryani et al. [30, 31] (Figure 6.4 and 6.5). 

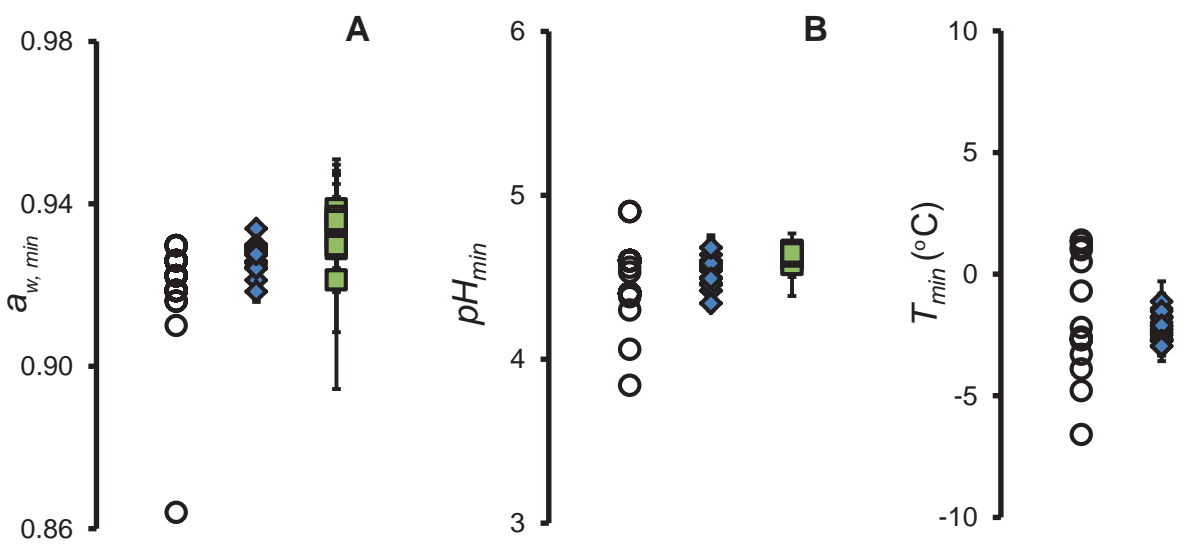

Figure 6.4: Variability in the cardinal growth parameters $\mathrm{a}_{\mathrm{w} \text {, min }}(\mathrm{A}), \mathrm{pH}_{\min }(\mathrm{B})$ and $\mathrm{T}_{\min }$ (C) caused by population heterogeneity (white: literature data [37-39], blue: 20 strains Aryani et al. [31], green: LO28 WT and eight acid resistant variants). The error bars represent the $95 \%$ confidence interval of the parameter estimation.

From Figure 6.4 it is clear that the cardinal growth parameters of the variants mostly fall within the range of the literature data with a slight bias upward. This indicates that when predicting growth for L. monocytogenes under different processing conditions and combinations of hurdles, as was done in Chapter 5, the range of literature parameters will provide a safe range. However, when looking at the range of $20 \mathrm{~L}$. monocytogenes strains compared to the range of the eight acid resistant LO28 variants, it can be seen that the 20 strains comprise a larger variability than thevariants for $\mathrm{pH}_{\min }$. The variability is comparable for $\mathrm{a}_{\mathrm{wmin}}$ but is split in two groups for $\mathrm{T}_{\min }$ giving the variants a larger variability than the 20 strains. Interestingly, the group with the higher $\mathrm{T}_{\min }$ mostly consists of the rpsU variants. Also the $95 \%$ confidence interval for the parameter estimates is larger in the variants, most likely due to less data points to which the secondary growth models were fitted compared to Aryani et al. [31]. The D-values obtained in Chapter 5 for variant 14 and the WT were benchmarked with literature data and the $20 \mathrm{~L}$. monocytogenes strains as well. The $20 \mathrm{~L}$. monocytogenes strains were shown to already explain most of the variability reported in literature. The bandwidth of D-values for the 20 strains, including growth history was the same as for the literature data. When comparing the D-values of the WT and variant 14 to the 20 strains and literature strains, it can be seen that they fall within the range of the literature but are in the upper range of the 20 strains (Figure 6.5). 


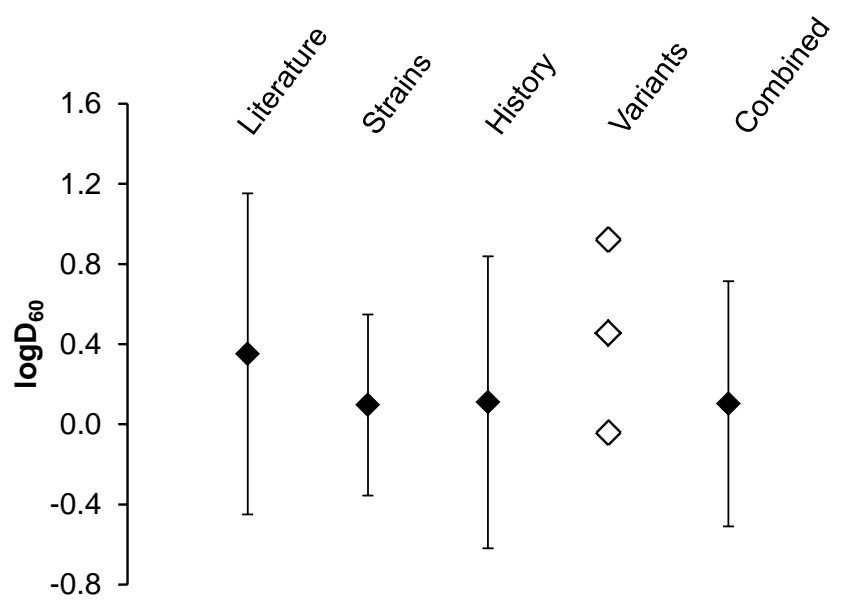

Figure 6.5: Variability in heat resistance, represented by $\log _{60}\left(\log _{10}\right.$ min)values from literature, 20 strains, three strains including growth history, variant 14 and the sensitive and resistant fraction of $\mathrm{LO} 28$ WT, and the combination of strain, history and variants. Error bars represent the 95\% prediction interval.

The upper data point in Figure 6.5 represents the resistant fraction of the WT, the lower data point the sensitive fraction of the WT and the middle point variant 14. The variability introduced by population heterogeneity of one strain is similar to the variability of 20 strains and therefore population heterogeneity is quantitatively clearlyanimportantvariability factor as well, which should betaken into consideration when it comes to stress survival predictions. Including individual cell heterogeneity into predictive models has received increasing attention in the last years, although most studies focus on growth rather than inactivation. Individual cell heterogeneity has been shown to contribute to variability in growth and inactivation, thereby affecting the outcome of predictive models [41]. It was shown that especially for small populations of less than $100 \mathrm{cfu}$, as is often the case in food products, the variability in D-value significantly increases as a result of individual cell heterogeneity [41]. One way of including variability into predictive models is to evaluate and prioritize different variability factors, as done in Figure 6.4 and Figure 6.5. Subsequently, a deterministic approach can be used in which the upper $95 \%$ confidence interval of the factors contributing most to variability are taken into consideration to make failsafe predictions. Another approach, as proposed by Aspridou and Koutsoumanis [41] is especially useful for predicting the behaviour of small populations. This involves a stochastic modelling approach in which different variability factors are included in the probability distribution. 


\section{Translation to other strains and species}

\section{Diversity in other strains}

L. monocytogenes LO28 is not amongst the most resistant L. monocytogenes strains [30] but its stable stress resistant variants show resistance levels that are comparable to those of strains displaying high heat resistance (see Fig 6.5). Therefore it is an interesting point of discussion if this phenomenon is only observed in domesticated labstrains orifthesamecan beobservedin strains thatarefound in food products, food processing environments or clinical isolates. The rpsU variant generally displayed between 2-3 times higher D-values than the WT, but the D-value of late-exponential phase cells at $55^{\circ} \mathrm{C}$ was 8 times higher. Although not evaluated in this thesis, it is a possibility that naturally higher heat resistant strains, for example strain L6 [30] also display a similar population diversity and presence of stable stress resistant variants. In case that the D-values of such variants are in this order of magnitude higher than the L6 WT D-values, they would be outside the 95\% prediction interval based on literature data of Figure 6.5. The presence of stable resistant variants in populations of such intrinsic resistant strains would increase the range that would need to be taken into account in predictive models and risk assessments. Although most detailed information on stable stress resistant variants is available for strain LO28, also some other L. monocytogenes strains were shown to display population diversity. HHP resistant variants were isolated from strain LO28, EGDe and Scott A [7, 8, 42], heat resistant variants from LO28 and EGDe [6] and also acid resistant variants were isolated from LO28 (this thesis) and EGDe (unpublished data). Both ctsR and rpsU mutations were shown to be present in stress resistant variants of strain LO28 (Figure 6.1) and EGDe (unpublished data) and ctsR mutations were present in Scott A HHP resistant variants [10, 42]. The presence of these different variants across different types of stress and different L. monocytogenes strains suggests that the generation of stress resistant variants, through introduction of mutations, could be a general strategy of the organism to prepare for survival under different adverse conditions. Especially with the potentially hyper-mutable regions in ctsR and rpsU, which are also present in other L. monocytogenes strains, it is not unlikely that the same strategy of survival is deployed by other strains as well. Given the low frequency of the mutations found $\left(5 \cdot 10^{-7}\right.$ for the rpsU variants), this strategy would only be effective at very high population density. On the other hand, it has been suggested that mutation rates can be affected by culture history and environmental conditions [43]. It can also be argued that laboratory strains and food isolates or clinical isolates have a different genetic elasticity and that strains that have survived the complete transmission cycle or are present in very specific niches in food production lines, are already genetically adapted to more stressful environments and 
therefore show less population diversity. We have attempted to get more insight in the relative magnitude of population diversity and the presence of stress resistant variants in other L. monocytogenes strains in several ways. Firstly, it was attempted to construct a $\Delta$ rpsU deletion mutant in four different L. monocytogenes strains to evaluate the impact of loss of RpsU function on other strains as well and to verify the role of rpsU in stress resistance. However, multiple attempts with different strategies, constructs and vectors, did not result in successful mutant construction. Also, the presence of stress resistant variants in other L. monocy togenes strains was evaluated in the same way as was done for $\mathrm{LO} 28$ in Chapter 2. Next to assessment of inactivation parameters, the presence of acid resistant variants was evaluated for late-exponential phase cells of strains F2365 and H7764 upon exposure to pH 3.0. Both strains showed significant tailing and strain $\mathrm{H} 7764$ had a significantly higher resistant fraction than strain $\mathrm{F} 2365$ and $\mathrm{LO} 28$, which by itself already can have an impact on food safety. For strain F2365, one stable highly acid resistant variant was isolated after 50 min exposure to $\mathrm{pH} 3.0$ out of the 60 tested survivors from the tail. This variant showed a multiple stress resistant phenotype, as previously observed for the ctsR and rpsU variants of LO28 and EGDe, but genomic analysis of the rpsU and ctsR regions revealed no mutations in these regions. Also some variants with a slightly increased stress resistant phenotype were isolated for both strains ( 5 out of 60 for F2365 and 2 out of 50 for $\mathrm{H7764}$ ), some of which also displayed a reduced growth rate, like observed for the ctsR and rpsU variants as well. However, these variants neither had a mutation in any of these two genes. The fact that also L. monocytogenes food isolates seem to display phenotypic and genotypic heterogeneity contributes to the concept that this is a general strategy for this pathogen to survive in different environments. The fact that only a limited number of variants was found in these other strains and that no ctsR and rpsU variants were found, does not necessarily imply that there is less diversity within these strains. The method applied here was based on isolation of survivors from a random time point in the tail of theinactivation curve. However, as is clear from Chapter 5 and Figure 6.3, the probability of isolating variants highly depends on theinactivation kinetics and variants can easily be missed when the 'wrong' sampling point is chosen. To learn more about the abundance of these mutations and the effect on population dynamics, a different approach might be appropriate. The current method is very labour intense and development of a high-throughput screening method would be very beneficial to better evaluate the diversity within different $\mathrm{L}$. monocytogenes strains. 


\section{Diversity in other species}

The presence of stress resistant variants is not limited to L. monocytogenes but also reported for other microbial species. Most of these other stress resistant variants have been isolated upon HHP exposure and have been reported for Escherichia coli [44], Saccharomyces cerevisiae [45] and Staphylococcus aureus [24]. In addition, Salmonella stress resistant variants have been isolated after repeated cycles of pulsed electric field (PEF) exposure [46] and acid exposure [47]. Interestingly, none of these variants were reported to bectsR variants, whereas in most cases it was evaluated if ctsR was intact or not. The presence of rpsU mutants was not evaluated since the knowledge on the potential role of rpsU in L. monocytogenes population diversity was not reported before our study and was therefore not specifically tested for. However, some information is available on the role of ribosomal protein S21 in other species. A Bacillus subtilis rpsU mutant has been characterized in detail by Akanuma et al. [17] and in our laboratory (unpublished data). The B. subtilis $\Delta r p s U$ mutant showed a reduced growth rate, which was more pronounced at lower temperatures, as was also observed for the L. monocytogenes rpsU variants. Motility was affected in the B. subtilis $\Delta$ rpsU mutant, but not in the L. monocytogenes variants. However, the microarray analysis showed downregulation of all the motility and flagella related genes in the L. monocytogenes rpsU variants compared to the WT (Figure 6.1), suggesting a potential role of rpsU in motility in L. monocytogenes as well. The B. subtilis $\Delta$ rpsU mutant showed a defect in cell separation, resulting in the formation of long chains [48] which was not observed in the L. monocytogenes variants. The role of $\mathrm{rpsU}$ in stress resistance in $\mathrm{B}$. subtilis was not described in literature. We therefore evaluated the heat and acid resistance of the $\Delta$ rpsU mutant of Akanuma et al. [17] and found no significant difference in stress resistance between the mutant and the WT, suggesting no significant role of rpsU in stress resistance in B. subtilis. Thus, it seems that mutations in rpsU are not a general mechanism of bacteria to generate diversity and a small stress resistant subpopulation but it would be interesting to evaluate the effect of rpsU mutations in other species as well. If the population diversity is caused by random mutations it is of course possible that also in B. subtilis these mutations occur in rpsU, as the similarities are very high between the rpsU sequences of the two organisms, including the repeats in which the mutations in L. monocytogenes are observed. On the other hand, the surrounding genes are different for L. monocytogenes and B. subtilis, which could explain the different effect on cellular level and the differences between the role of rpsU in L. monocytogenes and B. subtilis. The reduced growth rate, combined with an equal level of stress resistance as the WT makes that this mutation does not give a competitive advantage in B. subtilis and it is highly conceivable that thesemutations will notbecome predominantly present in the population. The occurrence of other mutations that do lead to stress resistance and possible competitive advantage cannot be excluded, but this remains a topic for future research. 


\section{Population heterogeneity: Survival strategy and impact on food safety}

Several survival and adaptation strategies of L. monocytogenes to facilitate its transmission from soil to humans have been discussed in Chapter 1. From the work presented in this thesis, as well as previously performed research on this topic, it can be concluded that also generation of population diversity can be considered a survival strategy. This may have implications for food safety, mainly in minimally processed foods. Chapter 5 clearly shows that the population composition might change following sequential growth and inactivation events, with resistant variants eventually constituting a larger fraction of the population. Therefore, it is not unlikely that variants become the dominant population in food production lines. As suggested by the results in Chapter 5, this highly depends on the number and order of environmental niches encountered. This is illustrated by the large impact of growth temperature on subsequent stress survival and the effect of stress duration and intensity on recovery time and subsequent outgrowth. It is well described in literature that all these different factors play a role in stress survival and recovery $[49,50]$. These factors may be different for different types of variants and therefore the environmental conditions determine if a certain type of variant has the potential to dominate a population in food production lines. From the work described in this thesis it is clear that the benefit of the increased resistance can be considered a trade-off, which is in most cases reflected by a reduced growth rate. Also, as can be seen from Chapter 4, in contrast to the WT some acid resistant variants do not show a strong acid adaptation anymore, resulting in similar acid stress survival capacity of WT and variants after pre-incubation at mildly acidic conditions prior to lethal acid stress exposure. Most likely the equal survival of the variants as the WT in mixed biofilms with Lactobacillus plantarum was a consequence of this lack of acid adaptation in these variants. On the other hand, the variants which showed an acid adaptation response in planktonic state also showed increased survival in these acidic biofilms. The highly increased survival of some variants in these biofilms can be considered a potential risk for persistence (Chapter 4) and thus a potential threat for food safety. Interestingly, the variants showing the best survival in the biofilms were the ones that were shown to score lower on the virulence indicators in Chapter 3, highlighting again the trade-off of these variants and the impact of the environmental conditions on the food safety risk. Another factor to be taken into consideration is that these variants are equally capable of forming single species biofilms as the WT. Conditions within a biofilm are often stressful and therefore it is not only a risk that these variants are capable of forming biofilms, but also that they might be generated within biofilms. It was shown by van der Veen et al. that continuous-flow biofilms induced the generation of rifampicin-resistant variants by 
activation of the SOS-response [51]. Such conditions may also lead to a higher fraction of food preservation stress-resistant variants, which in turn pose an additional food safety risk upon dispersal from biofilms. However, in assessing the food safety risk of stress resistant variants, it is also important to consider their virulence potential. It was shown by van Boeijen et al. [12] that none of the variants showed increased virulence in a mouse model and that some even showed a lower virulence. As shown in Figure 6.2, two of the previously isolated HHP resistant variants appeared to be rpsU variants. One of these variants was included in the assay of Van Boeijen et al. and showed reduced virulence potential, suggesting that the rpsU variants pose a lower public health hazard than the WT. However, the increased acid resistance may lead to increased stomach survival can also be considered as virulence factor, which was not taken into consideration in the mouse model. In the current work, the variants have been isolated after a single stress exposure, but also multiple cycles of stress exposure have been shown to result in the isolation of stress resistant variants [6]. Both, single and repeated stress exposure can be encountered in food industry, and it is therefore important to realise that such conditions can lead to selection for stress resistant variants. Another challenge that remains topic for future research is the prediction of variant generation within populations. The complexity of most natural and food processing environments, providing bacterial cells with different degrees of intrinsic stress resistance, and the fact that some conditions may select for variants while others might induce generation of variants, make this a very challenging topic of research. With the knowledge generated in this thesis, literature data and principles of evolution, the model in Figure 6.6 is proposed.

The model illustrates that mutations are either randomly generated, potentially with a higher probability in hyper-mutable regions, or induced by stress exposure for example by activation of the SOS response. The population thus exists of mainly WT cells, but also some cells with a potentially beneficial mutation are present. If a mutation is beneficial depends on the species and potentially also on the strain and the conditions that are subsequently encountered by the cells. The cells with a potentially beneficial mutation will either become the dominant population (rpsU under severe acid stress, ctsR under heat stress), stay present in the low fraction

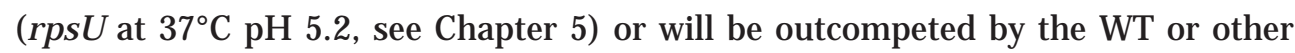
subpopulations (rpsU at low temperature). The proposed model considers the presence of stress resistant variants a survival strategy for L. monocytogenes as the genetic diversity prepares the population for many environmental conditions that can be encountered during the transmission from soil to the human gastro-intestinal tract and all the niches in between. 


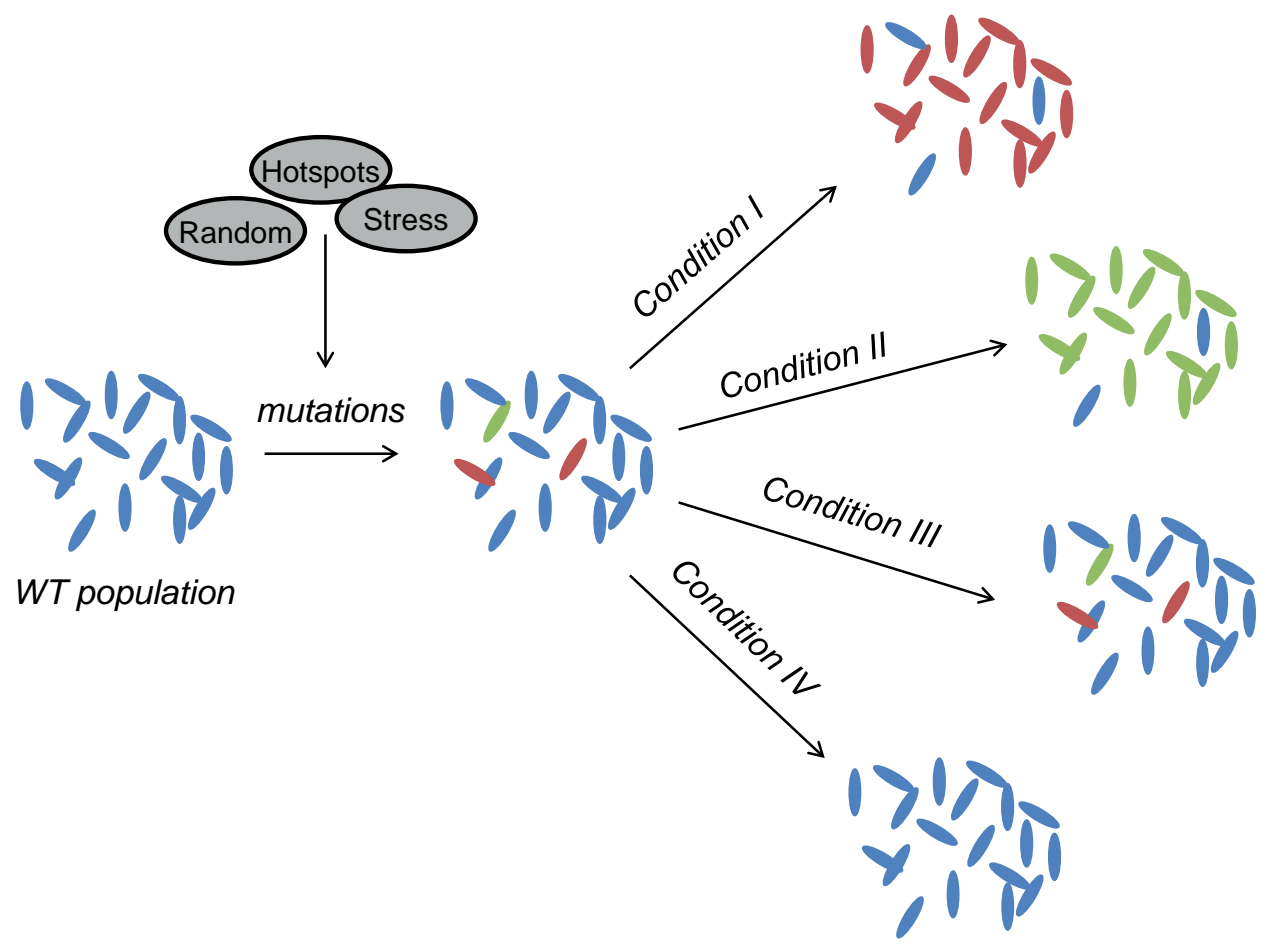

Figure 6.6: Proposed model on diversity within bacterial populations affected by environmental conditions. Diversity is generated within the population by the introduction of mutations, which can have several causes. The environmental conditions subsequently encountered by the population determine if a certain mutation is selected for or if variants are outcompeted by the main population or any of the other sub-populations.

\section{Conclusions and future perspectives}

This thesis has provided insight in the behaviour of stress resistant variants of $\mathrm{L}$. monocytogenes and quantified the potential impact of the presence of stress resistant variants on food safety using a food chain modelling approach. It was shown that also acid stress leads to selection for stable stress resistant variants of L. monocytogenes LO28. This finding, combined with a phenotypic and genotypic characterization, and the knowledge on previously isolated stress resistant variants after heat and HHP exposure, highlighted the population diversity of L. monocytogenes. It can be concluded that the environmental conditions affect the population composition and determine if stable resistant variants are selected for. The approach that was followed in this thesis was a combination of predictive modelling and acquisition of mechanistic knowledge, which proved to be a powerful combination to evaluate the potential impact of stress resistant variants on food safety. The inactivation kinetics combined with the knowledge on the fraction harbouring the same mutation, and 
therefore displaying the same kinetic behaviour, helped to make predictions on the processing conditions that select for this specific type of variant. Although a significant amount of mechanistic knowledge was generated during the course of this research, there is still a number of variants for which the underlying mechanisms of their stress resistant phenotypes are unknown. Future research will focus on unravelling the mechanisms behind these variants, in order to get a complete picture of the different mutations leading to a stress resistant phenotype and which conditions select for these variants. Another topic for future research is to evaluate if the mutations found in the variants are random or if they are directed by the organism through mutation hotspots or other mechanisms. More mechanistic knowledge on the generation of population diversity might allow for prediction of presence of certain stress resistant variants. At the moment it is still challenging to link outbreak strains to specific food products or niches in food production lines. Although the phenotypes of ctsR and rpsU variants are very different from the WT, they can be genetically very similar to the WT and more similar than can be discriminated by most of the currently used molecular typing methods. Recent advances and increased accuracy in whole genome sequencing (WGS) will undoubtedly lead to further unravelling of the genetic diversity within L. monocytogenes populations [52]. Deeper sequencing with higher resolution and linking genomic information to phenotypic information will allow for faster screening for potentially stress resistant phenotypes within processing plants [53]. Broader knowledge on genotypes and the corresponding phenotypes will also give moreinsight in the potential of strains and corresponding variants to become persistently present in food production lines. Another point of interest for future research is the translation of the knowledge available for L. monocytogenes LO28 to other strains. A more general and quantitative picture of population diversity within L. monocytogenes will allow for better decision making if population diversity and the presence of tailing is to be taken into account as variability factor upon implementation of modified or new food processing regimes and technologies. Realistic predictions that allow for integration of population diversity and the effect of environmental conditions on the population composition still poses challenges that need to be addressed in future research.

Overall, it can be concluded that genetic diversity is widely present in L. monocytogenes populations and that the environmental conditions determine if variants are selected for, and their subsequent fate in the food chain. Better understanding of the mechanisms underlying increased resistance might provide tools for better control of L. monocytogenes. Quantitative knowledge on behaviour and generation of this genetic diversity allows for incorporation in predictive models and risk assessments. Both quantitative and mechanistic knowledge on stress resistant variants of L. monocytogenes are of great importance in minimal processing which aims at producing microbiologically stable food products with maintenance of nutritional value and better sensory properties. 


\section{References}

1. Currie, A., J.M. Farber, C. Nadon, D. Sharma, Y. Whitfield, C. Gaulin, . . . U. Sierpinska, Multi-province listeriosis outbreak linked to contaminated deli meat consumed primarily in institutional settings, Canada, 2008. Foodborne Pathogens and Disease, 2015. 12(8): p. 645-652.

2. Johnsen, B.O., E. Lingaas, D. Torfoss, E.H. Strøm, and I. Nordøy, A large outbreak of Listeria monocytogenes infection with short incubation period in a tertiary care hospital. J ournal of Infection, 2010. 61(6): p. 465-470.

3. Koch, J., R. Dworak, R. Prager, B. Becker, S. Brockmann, A. Wicke, . . . K. Stark, Large listeriosis outbreak linked to cheese made from pasteurized milk, Germany, 2006-2007. Foodborne Pathogens and Disease, 2010. 7(12): p. 1581-1584.

4. McCollum, J.T., A.B. Cronquist, B.J . Silk, K.A. J ackson, K.A. O'Connor, S. Cosgrove, . . . B.E. Mahon, Multistate outbreak of listeriosis associated with cantaloupe. New England J ournal of Medicine, 2013. 369(10): p. 944-953.

5. Mead, P.S., E.F. Dunne, L. Graves, M. Wiedmann, M. Patrick, S. Hunter, . . . S. Zansky, Nationwide outbreak of listeriosis due to contaminated meat. Epidemiology and Infection, 2006. 134(4): p. 744-751.

6. Van Boeijen, I.K.H., C. Francke, R. Moezelaar, T. Abee, and M.H. Zwietering, Isolation of highly heat-resistant Listeria monocytogenes variants by use of a kinetic modeling-based sampling scheme. Applied and Environmental Microbiology, 2011. 77(8): p. 2617-2624.

7. Van Boeijen, I.K.H., R. Moezelaar, T. Abee, and M.H. Zwietering, Inactivation kinetics of three Listeria monocytogenes strains under high hydrostatic pressure. J ournal of Food Protection, 2008. 71(10): p. 2007-2013.

8. Karatzas, K.A.G. and M.H.J. Bennik, Characterization of a Listeria monocytogenes Scott A isolate with high tolerance towards high hydrostatic pressure. Applied and Environmental Microbiology, 2002. 68(7): p. 3183-3189.

9. Karatzas, K.A.G., V.P. Valdramidis, and M.H.J . Wells-Bennik, Contingency locus in ctsR of Listeria monocytogenes Scott A: A strategy for occurrence of abundant piezotolerant isolates within clonal populations. Applied and Environmental Microbiology, 2005. 71(12): p. 8390-8396.

10. Karatzas, K.A.G., J.A. Wouters, C.G.M. Gahan, C. Hill, T. Abee, and M.H.J. Bennik, The CtsR regulator of Listeria monocytogenes contains a variant glycine repeat region that affects piezotolerance, stress resistance, motility and virulence. Molecular Microbiology, 2003. 49(5): p. 1227-1238.

11. Van Boeijen, I.K.H., A.A.E. Chavaroche, W.B. Valderrama, R. Moezelaar, M.H. Zwietering, and T. Abee, Population diversity of Listeria monocytogenes LO28: Phenotypic and genotypic characterization of variants resistant to high hydrostatic pressure. Applied and Environmental Microbiology, 2010. 76(7): p. 2225-2233.

12. Van Boeijen, I.K.H., P.G. Casey, C. Hill, R. Moezelaar, M.H. Zwietering, C.G.M. Gahan, and T. Abee, Virulence aspects of Listeria monocytogenes LO28 high pressure-resistant variants. Microbial Pathogenesis, 2013. 59-60: p. 48-51.

13. Cotter, P.D. and C. Hill, Surviving the acid test: Responses of Gram-positive bacteria to low $\mathrm{pH}$. Microbiology and Molecular Biology Reviews, 2003. 67(3): p. 429-453.

14. Patchett, R.A., Watson, N., Fernandez, P.S., Kroll, R.G., The effect of temperature and growth rate on the susceptibility of Listeria monocytogenes to environmental stress conditions. Letters in Applied Microbiology, 1996. 22: p. 121-124.

15. Karatzas, K.A.G., L. Suur, and C.P. O'Byrne, Characterization of the intracellular glutamate decarboxylase system: Analysis of its function, transcription, and role in the acid resistance of various strains of Listeria monocytogenes. Applied and Environmental Microbiology, 2012. 78(10): p. 35713579. 
16. Feehily, C. and K.A.G. Karatzas, Role of glutamate metabolism in bacterial responses towards acid and other stresses. J ournal of Applied Microbiology, 2013. 114(1): p. 11-24.

17. Akanuma, G., H. Nanamiya, Y. Natori, K. Yano, S. Suzuki, S. Omata, . . . F. Kawamura, Inactivation of ribosomal protein genes in Bacillus subtilis reveals importance of each ribosomal protein for cell proliferation and cell differentiation. J ournal of Bacteriology, 2012. 194(22): p. 62826291.

18. Drake, J.W., Chaos and order in spontaneous mutation. Genetics, 2006. 173(1): p. 1-8.

19. Galhardo, R.S., P.J. Hastings, and S.M. Rosenberg, Mutation as a stress response and the regulation of evolvability. Critical Reviews in Biochemistry and Molecular Biology, 2007. 42(5): p. 399-435.

20. Rando, O.J . and K.J . Verstrepen, Timescales of genetic and epigenetic inheritance. Cell, 2007. 128(4): p. 655-668.

21. Maharjan, R., S. Nilsson, J . Sung, K. Haynes, R.E. Beardmore, L.D. Hurst, . . . I. Gudelj, The form of a trade-off determines the response to competition. Ecology Letters, 2013. 16(10): p. 1267-1276.

22. Van Belkum, A., S. Scherer, L. Van Alphen, and H. Verbrugh, Short-sequence DNA repeats in prokaryotic genomes. Microbiology and Molecular Biology Reviews, 1998. 62(2): p. 275-293.

23. Rocha, E.P.C., I. Matic, and F. Taddei, Over-representation of repeats in stress response genes: A strategy to increase versatility under stressful conditions? Nucleic Acids Research, 2002. 30(9): p. 1886-1894.

24. Karatzas, K.A.G., A. Zervos, C.C. Tassou, C.G. Mallidis, and T.J . Humphrey, Piezotolerant small-colony variants with increased thermotolerance, antibiotic susceptibility, and lowinvasiveness in a clonal Staphylococcus aureus population. Applied and Environmental Microbiology, 2007. 73(6): p. 1873-1881.

25. van der Veen, S. and T. Abee, Bacterial SOS response: A food safety perspective. Current Opinion in Biotechnology, 2011. 22(2): p. 136-142.

26. Van der Veen, S., S. van Schalkwijk, D. Molenaar, W.M. de Vos, T. Abee, and M.H.J . Wells-Bennik, The SOS response of Listeria monocytogenes is involved in stress resistance and mutagenesis. Microbiology, 2010. 156(2): p. 374-384.

27. Schlacher, K. and M.F. Goodman, Lessons from 50 years of SOS DNA-damage-induced mutagenesis. 2007. 8(7): p. 587-594.

28. Geeraerd, A.H., V. Valdramidis, and J.F. Van Impe, GInaFiT, a freeware tool to assess nonlog-linear microbial survivor curves. International J ournal of Food Microbiology, 2005. 102(1): p. 95-105.

29. van Boekel, M.A.J .S., Kinetic modeling of reactions in foods. 2009: CRC Press.

30. Aryani, D.C., H.M.W. den Besten, W.C. Hazeleger, and M.H. Zwietering, Quantifying variability on thermal resistance of Listeria monocytogenes. International Journal of Food Microbiology, 2015. 193(0): p. 130-138.

31. Aryani, D.C., H.M.W. den Besten, W.C. Hazeleger, and M.H. Zwietering, Quantifying strain variability in modeling growth of Listeria monocytogenes. International J ournal of Food Microbiology, 2015. 208: p. 19-29.

32. De J esús, A.J . and R.C. Whiting, Thermal inactivation, growth, and survival studies of Listeria monocytogenes strains belonging to three distinct genotypic lineages. J ournal of Food Protection, 2003. 66(9): p. 1611-1617.

33. Lianou, A. and K.P. Koutsoumanis, Evaluation of the strain variability of Salmonella enterica acid and heat resistance. Food Microbiology, 2013. 34(2): p. 259-267.

34. Mackey, B.M., C. Pritchet, A. Norris, and G.C. Mead, Heat resistance of Listeria: Strain differences and effects of meat type and curing salts. Letters in Applied Microbiology, 1990. 10(6): p. 251-255.

35. Sorqvist, S., Heat resistance of different serovars of Listeria monocytogenes. J ournal of Applied Bacteriology, 1994. 76(4): p. 383-388. 
36. Whiting, R.C. and M.H. Golden, Variation among Escherichia coli O157:H7 strains relative to their growth, survival, thermal inactivation, and toxin production in broth. International J ournal of Food Microbiology, 2002. 75(1-2): p. 127-133.

37. Augustin, J.C. and V. Carlier, Mathematical modelling of the grouth rate and lag time for Listeria monocytogenes. International J ournal of Food Microbiology, 2000. 56(1): p. 29-51.

38. Coroller, L., D. Kan-King-Yu, I. Leguerinel, P. Mafart, and J.M. Membré, Modelling of growth, growth/no-growth interface and nonthermal inactivation areas of Listeria in foods. International J ournal of Food Microbiology, 2012. 152(3): p. 139-152.

39. Van der Veen, S., R. Moezelaar, T. Abee, and M.H.J. Wells-Bennik, The grouth limits of a large number of Listeria monocytogenes strains at combinations of stresses show serotype- and niche-specific traits. J ournal of Applied Microbiology, 2008. 105(5): p. 1246-1258.

40. Van Asselt, E.D. and M.H. Zwietering, A systematic approach to determine global thermal inactivation parameters for various food pathogens. International J ournal of Food Microbiology, 2006. 107(1): p. 73-82.

41. Aspridou, Z. and K.P. Koutsoumanis, Individual cell heterogeneity as variability source in population dynamics of microbial inactivation. Food Microbiology, 2015. 45, Part B: p. 216-221.

42. J oerger, R.D., H. Chen, and K.E. Kniel, Characterization of a spontaneous, pressure-tolerant Listeria monocytogenes Scott A ctsR deletion mutant. Foodborne Pathogens and Disease, 2006. 3(2): p. 196-202.

43. Ryall, B., G. Eydallin, and T. Ferenci, Culture history and population heterogeneity as determinants of bacterial adaptation: the adaptomics of a single environmental transition. Microbiology and Molecular Biology Reviews, 2012. 76(3): p. 597-625.

44. Hauben, K.J .A., D.H. Bartlett, C.C.F. Soontjens, K. Cornelis, E.Y. Wuytack, and C.W. Michiels, Escherichia coli mutants resistant to inactivation by high hydrostatic pressure. Applied and Environmental Microbiology, 1997. 63(3): p. 945-950.

45. Fujii, S., H. Iwahashi, K. Obuchi, T. Fujii, and Y. Komatsu, Characterization of a barotolerant mutant of the yeast Saccharomyces cerevisiae: importance of trehalose content and membrane fluidity. FEMS Microbiology Letters, 1996. 141(1): p. 97-101.

46. Sagarzazu, N., G. Cebrián, R. Pagán, S. Condón, and P. Mañas, Emergence of pulsed electric fields resistance in Salmonella enterica serovar Typhimurium SL1344. International J ournal of Food Microbiology, 2013. 166(2): p. 219-225.

47. Karatzas, K.A.G., P.M. Hocking, F. J ørgensen, K. Mattick, S. Leach, and T.J . Humphrey, Effects of repeated cycles of acid challenge and growth on the phenotype and virulence of Salmonella enterica. J ournal of Applied Microbiology, 2008. 105(5): p. 1640-1648.

48. Takada, H., M. Morita, Y. Shiwa, R. Sugimoto, S. Suzuki, F. Kawamura, and H. Yoshikawa, Cell motility and biofilm formation in Bacillus subtilis are affected by the ribosomal proteins, S11 and S21. Bioscience, Biotechnology, and Biochemistry, 2014. 78(5): p. 898-907.

49. Leguérinel, I., I. Spegagne, O. Couvert, L. Coroller, and P. Mafart, Quantifying the effects of heating temperature, and combined effects of heating medium $\mathrm{pH}$ and recovery medium $\mathrm{pH}$ on the heat resistance of Salmonella typhimurium. International J ournal of Food Microbiology, 2007. 116(1): p. 88-95.

50. Mañas, P., R. Pagán, J. Raso, and S. Condón, Predicting thermal inactivation in media of different $\mathrm{pH}$ of Salmonella grown at different temperatures. International Journal of Food Microbiology, 2003. 87(1- 2): p. 45-53.

51. van der Veen, S. and T. Abee, Generation of variants in Listeria monocytogenes continuous-flow biofilms is dependent on radical-induced DNA damage and RecA-mediated repair. PLoS One, 2011. 6(12): p. e28590.

52. Nyarko, E.B. and C.W. Donnelly, Listeria monocytogenes: Strain heterogeneity, methods, and challenges of subtyping. J Food Sci, 2015. 80(12): p. M2868-78. 
53. Ortiz, S., V. López-Alonso, P. Rodríguez, and J.V. Martínez-Suárez, The connection between persistent, disinfectant-resistant Listeria monocytogenes strains from two geographically separate Iberian pork processing plants: Evidence from comparative genome analysis. Applied and Environmental Microbiology, 2016. 82(1): p. 308-317. 
Summary

Samenvatting Acknowledgements About the author List of publications Overview of training activities 



\section{Summary}

The increased consumer demand for fresh and healthy products has led to advances in minimal processing, which aims at finding the balance between microbiologically stable foods while maintaining the characteristics of fresh products. Bacterial stress response and heterogeneity therein is one of the biggest challenges posed by minimal processing. A heterogeneous stress response can be observed for example as tailing of inactivation curves upon stress exposure. The first, steep part of the inactivation curve represents the main, stress sensitive part of the population and the second, flatter part of the curve represents the small, resistant fraction of the population. Heterogeneity and resulting tailing can have several causes and can roughly be divided in transient and stably increased stress resistance. Transient increased resistance often has a phenotypic or epigenetic background and is lost when the cells are cultured and exposed to stress again. Stable increased stress resistance on the other hand is caused by alterations in the genome and therefore inheritable and stable in nature. The latter type of cells we refer to as stable stress resistant variants. Listeria monocytogenes is one of the most robust non-sporeforming foodborne pathogens. It can grow and survive under a wide range of environmental and foodprocessing conditions. The incidence of L. monocytogenes infections is low, but the severity of the disease and the high mortality rate make it one of the top three causes of death by foodborne disease. Also L. monocytogenes exhibits a heterogeneous response upon stress exposure which can be partially attributed to the presence of stable stress resistant variants. Adverse environments were shown to select for stable stress resistant variants. Tailing of L. monocytogenes inactivation curves upon stress exposure and conceivable roles of stress resistant variants has been well established and qualitative data is available for a number of variants isolated upon high hydrostatic pressure (HHP) and heat exposure. The objective of the research described in this thesis was to evaluate if L. monocytogenes population diversity and the presence of stable resistant variants is a general phenomenon that is observed upon different types of stress exposure, to get more insight in the mechanisms leading to increased resistance and to evaluate the ecological behaviour and potential impact on food safety of these stable resistant variants. The approach followed to reach the objective was to get more qualitative, quantitative and mechanistic knowledge on the behaviour of stable stress resistant variants and to extent the knowledge to another type of stress, namely acid stress. Acid stress was chosen as it is an important hurdle both in food preservation, as well as in stomach survival.

In Chapter 2, the non-linear inactivation kinetics of L. monocytogenes upon acid exposure were quantitatively described. A commonly used biphasic inactivation model was reparameterized, which improved the statistical performance of the 
model and resulted in more accurate estimation of the resistant fraction within L. monocytogenes WT populations. The observed tailing suggested that stable stress resistant variants might also be found upon acid exposure. Indeed, 23 stable acid resistant variants of $\mathrm{L}$. monocytogenes $\mathrm{LO}_{2} 8$ were isolated from the tail after exposure of late-exponential phase cells to $\mathrm{pH} 3.5$ for $90 \mathrm{~min}$, with different degrees of acid resistance amongst them. Increased acid resistance showed to be significantly correlated to reduced growth rate. Studying the growth boundaries of the WT and a representative set of variants indicated that the increased resistance of the variants was only related to survival of severe $\mathrm{pH}$ stress but did not allow for better growth or survival at mild $\mathrm{pH}$ stress.

In Chapter 3, these variants were further characterized phenotypically and cluster analysis was performed. This resulted in three clusters and four individual variants and revealed multiple-stress resistance, with both unique and overlapping features related to stress resistance, growth, motility, biofilm formation and virulence indicators. A higher glutamate decarboxylase (GAD) activity correlated with increased acid resistance. Whole genome sequencing of a set of variants was performed and revealed mutations in rpsU, encoding ribosomal protein S21. This rpsU mutation was found in all 11 variants comprising the largest phenotypic cluster, indicating a potential role of this ribosomal protein in stress resistance. Mutations in ctsR, which were previously shown to be responsible for increased resistance of heat and HHP resistant variants, were not found in the acid resistant variants. This underlined that large population diversity exists within one L. monocytogenes strain and that different adverse conditions drive selection for different variants.

Chapter 4 and 5 focussed on the ecological behaviour and potential impact of stress resistant variants on food safety. In Chapter 4, the performance in mixed species biofilms with Lactobacillus plantarum was evaluated, as well as their benzalkonium chloride (BAC) resistance in these biofilms. It was hypothesized that the acid resistant variants might also show better survival in biofilms with L. plantarum, which provide an acidic environment by lactose fermentation with $\mathrm{pH}$ values below the growth boundary of L. monocytogenes when biofilms mature. L. monocytogenes LO28 WT and eight acid resistant variants were capable of forming mixed biofilms with L. plantarum at $20^{\circ} \mathrm{C}$ and $30^{\circ} \mathrm{C}$ in $\mathrm{BHI}$ supplemented with manganese and glucose. Some of the variants were able to withstand the low $\mathrm{pH}$ in the mixed biofilms for a longer time than the WT and there were clear differences in survival between the variants which could not be correlated to (lactic) acid resistance alone. Adaptation to mild $\mathrm{pH}$ of liquid cultures during growth to stationary phase increased theacid resistance of some variants to a greater extent than of others, which could be correlated to increased survival in the mixed biofilms. There were no clear differences in BAC resistance between the wild type and variants in mixed biofilms. 
In Chapter 5, a set of robustness and fitness parameters of WT and variants was obtained and used to model their growth behaviour under combined mild stress conditions and to model their performancein a simulated food chain. This gave more insight in the trade-off between increased stress resistance and growth capacity. Predictions of performance were validated in single and mixed cultures by plate counts and by qPCR in which WT and an rpsU deletion variant were distinguished by specific primers. Growth predictions for WT and rpsU deletion variant were matching the experimental data generally well. Globally, thevariants are more robust than the WT but the WT grows faster than most variants. Validation of performance in a simulated food chain consisting of subsequent growth and inactivation steps, confirmed the trend of higher growth fitness and lower stress robustness for the WT compared to the rpsU variant. This quantitative data set provides insights into the conditions which can select for stress resistant variants in industrial settings and their potential persistence in food processing environments.

In conclusion, the work presented in this thesis highlights the population diversity of L. monocytogenes and the impact of environmental conditions on the population composition, which is of great importance for minimal processing. The work of this thesis resulted in more insight in the mechanisms underlying increased resistance of stress resistant variants and quantitative data on the behaviour of stress resistant variants which can be implemented in predictive microbiology and quantitative risk assessments aiming at finding the balance between food safety and food quality. 



\section{Samenvatting}

De laatste jaren is de vraag naar verse en gezonde levensmiddelen sterk toegenomen. Dit heeft geleid tot de ontwikkeling van diverse milde conserveringstechnieken die als doel hebben om te zorgen voor microbiologisch stabiele levensmiddelen, waarbij tegelijkertijd de karakteristieken van verse producten behouden blijven. Een grote uitdaging binnen de milde conservering is de stress respons van bacteriën en heterogeniteit in deze stress respons. Heterogeniteit in stress respons kan waargenomen worden als een 'staart' in deinactivatiecurvevan een populatiebacteriën als deze wordt blootgesteld aan stress. Het eerste, steile gedeelte van de inactivatie curve bestaat uit het grootste deel van de populatie, die gevoelig is voor de stress en het tweede, minder steile deel van de curve, bestaat uit een kleine sub-populatie die resistenter is tegen stress dan de rest van de populatie. Heterogeniteit en deze nietlineaire inactivatie kinetiek kan verschillende oorzaken hebben, en grofweg worden onderverdeeld in stabiele en niet-stabiele resistentie. Niet-stabiele resistentie heeft vaakeen fenotypische of epigenetischeachtergrond en gaatverloren op het moment dat de bacteriën opnieuw aan stress worden blootgesteld. Stabiele resistentie daarentegen wordt veroorzaakt door veranderingen in het DNA dieleiden tot verhoogde resistentie en daardoor is deze vorm van resistentie erfelijk en stabiel. Dit laatste type bacteriën noemen we stabiele stress resistente varianten.

Listeria monocytogenes is een van de meest robuuste, niet sporenvormende ziekteverwekkende bacteriën die we kunnen tegenkomen in ons voedsel. Deze bacterie kan groeien en overleven onder veel verschillende condities die voorkomen in zowel de natuurlijke omgeving, als ook in omgevingen waar levensmiddelen worden geproduceerd. L. monocytogenes infecties komen niet vaak voor, maar de ernst van de ziekte en de hoge mortaliteit die ermee samengaat, zorgen ervoor dat L. monocytogenes in de top drie van levensmiddelen gerelateerde doodsoorzaken staat. L. monocytogenes vertoont ook een heterogene stress respons, die voor een deel toegeschreven kan worden aan de aanwezigheid van stabiele stress resistente varianten. Het is aangetoond dat nadelige condities kunnen leiden tot selectie voor stress resistente varianten. Niet-lineaire inactivatie kinetiek als gevolg van blootstelling aan stress en de rol van de aanwezigheid van stress resistente varianten in L. monocytogenes populaties is vastgesteld in eerder onderzoek. Kwalitatieve data zijn beschikbaar voor een aantal varianten die geïsoleerd zijn uit de populatie na blootstelling aan hitte en aan hoge druk. Het doel van het onderzoek dat beschreven is in dit proefschrift was om te evalueren of populatie heterogeniteit en de aanwezigheid van stress resistente varianten een algemeen fenomeen is binnen L. monocytogenes populaties dat kan worden waargenomen bij blootstelling aan verschillende typen stress. Tegelijkertijd was het doel om meer inzicht tekrijgen in de mechanismen dieten grondslagliggen aan deverhoogdestress resistentievan devarianten en hetecologische 
gedrag en the potentiele impact op voedselveiligheid van deze stabiele varianten te evalueren. Deaanpak om dit doel te bereiken was om meer kwalitatieve, kwantitatieve en mechanistische kennis te vergaren over deze stress resistente varianten en om de bestaande kennis uit te breiden met een andere vorm van stress, namelijk zuurstress. Er is voor zuurstress gekozen omdat dit een belangrijke belemmerende factor is voor bacteriën, zowel als conserveringsstap in de levensmiddelenindustrie, als tijdens overleving in de lage $\mathrm{pH}$ van de maag.

In hoofdstuk 2 van dit proefschrift is de niet-lineare inactivatie kinetiek van L. monocytogenes tijdens zuurblootstelling kwantitatief beschreven. Een algemeen gebruikt bifasisch inactivatiemodel dat inactivatie van bacteriën kwantitatief beschrijft, is opnieuw geparameteriseerd en dit heeft de statistische prestatie van het model verbeterd. Hierdoor kan de resistente fractie binnen de L. monocytogenes wildtype (WT) populatie nauwkeuriger worden geschat. De waargenomen 'staart' in de inactivatiecurve suggereerde dat ook na zuurblootstelling stabiele resistente varianten geïsoleerd zouden kunnen worden. Dit bleek inderdaad het geval te zijn, en 23 stabiele resistente varianten zijn geïsoleerd na 90 minuten blootstelling vaneen laat-exponentiele cultuur aan $\mathrm{pH}$ 3.5. Onder de varianten waren verschillende niveaus van zuurresistentie. De verhoogde zuurresistentie was significant gecorreleerd aan verlaagde groeisnelheid. Na het bestuderen van de groeilimieten van het wildtype en een representatieve set varianten kon geconcludeerd worden dat de verhoogde zuurresistentie alleen gerelateerd was aan zuuroverleving en niet aan betere groei of overleving bij milde zuurstress.

In hoofdstuk 3 zijn de varianten verder fenotypisch gekarakteriseerd en een clusteranalyse is uitgevoerd. Dit resulteerde in drie clusters en vier individuele varianten. De fenotypering en clusteranalyse lieten zien dat de varianten meervoudig stress resistent zijn met zowel unieke als overlappende kenmerken op het gebied van stress resistentie, groei, motiliteit, biofilm vorming, en virulentie indicatoren. Een hogere glutamaat decarboxylase (GAD) activiteit was gecorreleerd met verhoogde zuurresistentie. DNA sequencing van het complete genoom van devarianten resulteerde in identificatie van mutaties in rpsU, een gen dat het ribosomale eiwit S21 codeert. Deze rpsU mutatie werd gevonden in alle 11 de varianten die in hetzelfde fenotypische cluster ingedeeld waren, wat een aanwijzing is dat dit ribosomale eiwit een rol speelt in stress resistentie. Mutaties in ctsR, die eerder gevonden waren en verantwoordelijk zijn voor verhoogde resistentie in hitte en hoge druk resistente varianten, werden niet gevonden in de zuurresistente varianten. Dit benadrukt dat een grote populatie diversiteit bestaat binnen een $\mathrm{L}$. monocytogenes stam en dat verschillende nadelige condities tot selectie van verschillende varianten kan leiden.

In hoofdstuk 4 en 5 ligt de focus op het ecologische gedrag van de varianten en de mogelijke impact van deze varianten op voedselveiligheid. In hoofdstuk 4 is gekeken 
naar deprestatievan devarianten ingemengdebiofilms samen methet bederforganisme Lactobacillus plantarum. Ook is gekeken naar de benzalkonium chloride (BAC) resistentie van de varianten in deze biofilms. De hypothese was dat de zuurresistente varianten wellicht beter zouden overleven in de biofilms met L. plantarum, dievoor een zureomgeving zorgen door de fermentatievan glucose tot melkzuur en daarmeezorgen voor een $\mathrm{pH}$ die onder de groeilimiet van L. monocytogenes ligt. L. monoctytogenes WT en acht zuurresistente varianten waren in staat om biofilms te vormen samen met L. plantarum bij $20^{\circ} \mathrm{C}$ en $30^{\circ} \mathrm{C}$ in $\mathrm{BHI}$ gesupplementeerd met mangaan en glucose. Een aantal varianten was in staat om de lage $\mathrm{pH}$ in de biofilms langer te weerstaan dan het WT en er waren duidelijke verschillen in overleving tussen de verschillende varianten die niet gecorreleerd konden worden aan enkel zuur resistentie of melkzuur resistentie. Aanpassing aan lage $\mathrm{pH}$ in vloeibare culturen tijdens de groei naar stationaire fase verhoogde de zuur resistentie van sommige varianten meer dan van anderen. Dit kon wel gecorreleerd worden aan verhoogde overleving in de gemengde biofilms. Er waren geen duidelijke verschillen in BAC resistentie tussen het WT en de varianten in de gemengde biofilms.

In hoofdstuk 5 is een serie groei en inactivatie parameters van het WT en de varianten verzameld en deze zijn vervolgens gebruikt om hun gedrag te modelleren onder gecombineerde milde stress condities, en om hun gedrag in een gesimuleerde voedselketen te voorspellen. Dit heeft meer inzicht gegeven in de 'trade-off' tussen verhoogde stress resistentie en groei capaciteit. Voorspellingen van het gedrag zijn gevalideerd in monoculturen en gemengde culturen van het WT en een variant door middel van plaattellingen en met qPCR waarbij het WT en een rpsU deletie variant onderscheiden werden door middel van specifieke primers. Groei voorspellingen van het WT en de rpsU variant beschreven de experimentele data relatief goed. Over het algemeen was de variant robuuster dan het WT maar het WT groeide sneller dan de varianten. Validatie van het gedrag in een gesimuleende voedselketen, bestaande uit opeenvolgende groei- en inactivatiestappen bevestigde de trend van hogere groeisnelheid maar verlaagde stress resistentie van het WT ten opzichte van de rpsU variant. Deze kwantitatieve dataset heeft meer inzicht gegeven in de condities die zouden kunnen leiden tot selectievoor stress resistentevarianten in delevensmiddelen industrie en de persistentie in de productieomgeving van levensmiddelen.

Het werk dat in dit proefschrift beschreven staat benadrukt depopulatie diversiteit van L. monocytogenes en de invloed van omgevingsfactoren op de samenstelling van de populatie, wat van groot belang is voor milde conserveringstechnieken. Dit onderzoek heeft geresulteerd in meer inzichten in de mechanismen die ten grondslag liggen aan de verhoogde stressresistentie van de varianten. Ook is er meer kwantitatieve data verkregen die het gedrag van de varianten beschrijft en kan worden gebruikt in voorspellende modellen en kwantitatieve risico analyses die tot doel hebben om de balans tussen voedselveiligheid en voedselkwaliteit te vinden. 



\section{Acknowledgements}

My work of the past five years has focussed on heterogeneity of bacterial populations and the advantage this can have in stressful environments. Also for me it was important to be part of a heterogeneous population during this $\mathrm{PhD}$ research and I am grateful for the motivation, inspiration and support I received from each individual the past five years to help me deal with sometimes stressful environments!

Als eerste wil ik mijn promotoren en co-promotor bedanken: Marcel, Tjakko and Heidy. Jullie expertise, kennis, ideeën en aanpak vullen elkaar op een geweldige manier aan. Marcel, ik heb ontzettend veel van je geleerd op het gebied van predictive microbiology. J ouw kritische blik, praktische aanpak en enthousiasme in het delen van je kennis zijn van ontschatbare waarde geweest voor mijn onderzoek. Tjakko, jouw explosies van ideeën hebben me altijd weten te inspireren en hebben tot veel interessante experimenten en resultaten geleid. Heidy, bedankt dat je de rol als co-promotor na 1.5 jaar hebt overgenomen. J ouw kritische commentaren op mijn manuscripten en experimentele set-up hebben enorm bijgedragen aan de kwaliteit ervan. In het rijtje van begeleiders hoort ook Roy thuis. Dit was jouw project en je enthousiasme werkte heel aanstekelijk op mij. Bedankt voor je hulp, inspiratie en steun tijdens de eerste 1.5 jaar!

Ik wil TI Food and Nutrition en de industriële partners bedanken voor het financieel mogelijk maken van dit project. Alle leden van het Food Safety \&Preservation team, bedankt voor de waardevolle discussies tijdens de expert meetings, gezelligheid tijdens WE days en andere team building activiteiten en leerzame momenten tijdens partners visits. Gerard, bedankt voor demanier waaropjehet modelling project geleid hebt, ik heb veel van je geleerd over project management en je steeds terugkerende vraag 'wat wil je worden als je groot bent?' heeft me geholpen om kritisch na te denken over wat ik wil en waar ik blij van word. Masja en Marjon, bedankt datjullie de rol als projectleider hebben overgenomen voor het laatste deel van het project.

Without excellent technical support, many experiments would not have been possible. Sacha en J os, bedankt voor de Bio-IT ondersteuning en meepubliceren van het genome sequencing werk. Mijn speciale dank gaat uit naar Jos voor het altijd geduldig beantwoorden van mijn Bio-IT vragen. Soraya, many thanks for all your efforts in the mutant construction work. It is a pity it did not work out, but you are a great teacher and I've learned a lot from you! Gerrieke, bedankt voor je bijdrage aan het mutanten werk. Marcel, bedankt voor je hulp bij het transcriptoom werk en alle overige trouble shooting op het gebied van moleculair werk tijdens de afgelopen jaren. Paula, thank you for working together on the mixed biofilms and for co-authoring the resulting paper. Mariëtte en Louise, bedankt dat jullie het FBR lab met mij en m'n studenten gedeeld hebben en bedankt dat jullie me op weg hebben 
geholpen op het lab tijdens de eerste maanden van mijn onderzoek. Ineke, bedankt voor het geweldige werk dat jij hebt gedaan voorlopend op mijn project en bedankt dat ik altijd bij je terecht kon met mijn inhoudelijke en praktische vragen.

A lot of work has been done by students that did a thesis on my project. Eva, Thomas, Kartika, Daan, Emel, Astrisia, Angela, Feyissa, Myriam and Roberto, thank you so much for your endless efforts and enthusiasm. It was a pleasure to work with each of you and I enjoyed seeing you grow. I wish you all the best for the future!

I am very grateful to Prof. Michiel Kleerebezem, Prof. Arie Havelaar, Dr. Kostas Koutsoumanis and Dr. J an-Willem Sanders for taking the time to critically review my thesis. Thank you for accepting the invitation to be part of my thesis committee.

Iliana, thank you for the beautiful cover design and for the lay-out of my thesis. You made our lives these last weeks a lot easier!

I also want to thank all the current and former colleagues of the Food Microbiology department for the wonderful time I've had these last years. I will miss the lunch breaks, interruptions, fun in the labs, $\mathrm{PhD}$ trips, lab outings, pubquiz evenings and all other moments we shared. Some extra words for my 'future PhD girls': Alicja, Monica and Hasmik, thank you for your friendship and sharing both happy and difficult PhD moments together. Diah, thank you for being my partner in crime in the Modelling project. It is great how our projects came together in the end. Gerda, bedankt voor je administratieve ondersteuning. Ingrid, bedankt voor het op orde houden van de keuken, de voorraden en de studenten. Ook bedankt aan Ben en het hele FTC team voor de fijne tijd tijdens de eerste 1.5 jaar van mijn project.

A special thank you goes to my paranimphs. Alicja, it totally makes sense that you are sitting next to me today. We have started our $\mathrm{PhD}$ 's together and I've really seen you grow during the past years. You are a great scientist and wonderful person. Jeroen, bedankt datjij ook naast me op het podium wil zitten vandaag. Ik ben ontzettend blij dat jij verder gaat met dit project. Bedankt voor jouw frisse blik op mijn werk, ik weet zeker dat je iets heel moois gaat maken van je eigen promotie onderzoek!

A very big thank you to all my friends, mijn familie, meine Swiegerfamilie. I feel very lucky to be surrounded by so many wonderful people that have supported me during this $\mathrm{PhD}$. All the dinners, drinks, weekends, game nights, sports, gardening and brewing sessions have helped me to stay with two legs on the ground and the moments to laugh and relax have been very important to me. Thank you, Danke schön, dankjulliewel!

Pap en mam, bedankt voor jullie vertrouwen in mijn kunnen en bedankt dat jullie achter mijn keuzes staan. J ullie hebben me geleerd om mijn ambities na te streven zolang die me gelukkig maken en er alles voor gedaan om te zorgen dat we een goede 
opleiding zouden kunnen volgen. Ellie, jouw heerlijke directe manier van dingen verwoorden maken me altijd aan het lachen en hebben me op de juste momenten geholpen te relativeren.

My dear Karsten, your love, support, and patience gave me the confidence to write this thesis. Along the road we have learned valuable lessons about finding a balance between ambitions and dreams. Your perseverance and never giving up have not only been important for your thesis, but definitely also for mine. We did it and I am extremely proud of us! 



\section{About the author}

Karin Inge Metselaar was born on 24 February 1985 in Amsterdam, the Netherlands. In 2003 she graduated from secondary school at Keizer Karel College in Amstelveen and continued with an Applied BSc Laboratory techniques at Hogeschool Larenstein in Velp. After completing the first year, she continued with a BSc in Nutrition and Health at Wageningen University and upon completion in 2008 she started the MSc program Food Safety at Wageningen University. Her major thesis was conducted at theLaboratory of food Microbiology and focused on thegermination and outgrowth of Bacillus cereus spores in reconstituted powdered infant formula. The internship that was part of the MSc program, was conducted at Unilever's Safety and Environmental Assurance Center (SEAC) in Colworth Science Park, UK. During this internship, she was working on growth and survival of vegetative pathogens in intermediate moisture foods. After graduating the MSc program in 2010, she has worked for 9 months at the Microbial Genomics group of TNO in Zeist. During this period she was working on a project about biofilm formation of thermophilic sporeformers in dairy environments. In 2011, Karin started her PhD program entitled, 'Quantitative and ecological aspects of Listeria monocytogenes population heterogeneity', at the Laboratory of Food Microbiology of Wageningen University. The results of this work are described in this thesis. Currently, Karin is working as Scientist Microbiology and Modelling at Corbion in Gorinchem. 



\section{List of Publications}

K.I. Metselaar, T. Abee, M.H. Zwietering, H.M.W. den Besten. Modeling and validation of ecological behaviour of Listeria monocytogenes wild type and stress resistant variants. Submitted for publication.

H.M.W. den Besten, D.C. Aryani, K.I. Metselaar, M.H. Zwietering. Microbial variability in growth and heat resistance of a pathogen and a spoiler: all variabilities are equal but some are more equal than others. Submitted for publication.

T. Abee, J . Koomen, K.I. Metselaar, M.H. Zietering, H.W.W. den Besten (2016). Impact of pathogen population heterogeneity and stress-resistant variants on food safety. Annual Review of Food Science and Technology 7: 439-456.

K.I. Metselaar, P. Saá Ibusquiza, A.R. Ortiz Camargo, M. Krieg, M.H. Zwietering, H.M.W. den Besten, T. Abee (2015). Performance of stress resistant variants of Listeria monocytogenes in mixed species biofilms with Lactobacillus plantarum. International J ournal of Food Microbiology 213:24-30.

K.I. Metselaar, H.M.W. den Besten, J. Boekhorst, S.A.F.T. van Hijum, M.H. Zwietering, T. Abee (2015). Diversity of acid stress resistant variants of Listeria monocytogenes and the potential role of ribosomal protein S21 encoded by rpsU. Frontiers in Microbiology 6:422.

K.I. Metselaar, H.M.W. den Besten, T. Abee, R. Moezelaar, M.H. Zwietering (2013). Isolation and quantification of highly acid resistant variants of Listeria monocytogenes. International Journal of Food Microbiology 166(3):508-14.

Y. Zhao, M.P.M. Caspers, K.I. Metselaar, P. de Boer, G. Roeselers, R. Moezelaar, M.N. Nierop Groot, R.C. Montijn, T. Abee, R. Kort (2013). Abiotic and microbiotic factors controlling biofilm formation by thermophilic sporeformers. Applied and Environmental Microbiology 79(18):5652-60. 



\section{Overview of completed training activities}

\section{Discipline specific activities}

\section{Courses}

Genetics and physiology of food associated microorganisms

VLAG, Wageningen

2010

Management of microbial hazards in foods

VLAG, Wageningen

2013

Reaction kinetics in food science

VLAG, Wageningen

2012

Advances Proteomics

VLAG, Wageningen

2013

Introduction Bioinformatics

TIFN, Wageningen

2012

Introduction Phenolink

TIFN, Wageningen

2013

\section{Meetings and conferences}

ICPMF8, oral presentation

Paris, France

2013

Workshop on Advances on Predictive Modeling and Quantitative

ESPCA, São Paulo, Brazil

2013

Microbial Risk Assessment of Foods

FoodMicro2014, oral presentation

Nantes, France

2014

ICPMF9, oral presentation

Rio de J aneiro, Brazil

2015

\section{General courses}

VLAG $\mathrm{PhD}$ week

VLAG, Wageningen

2011

Project and time management

WGS, Wageningen

2012

Philosophy and ethics in food science

VLAG, Wageningen

2013

Supervising thesis students

WGS, Wageningen

2014

Career perspectives

WGS, Wageningen

2015

\section{Optional courses}

Preparation of research proposal

WUR,Wageningen

2011

PhD trip 2012 J apan

FHM, Wageningen

2012

Organizing PhD trip 2012 Japan

FHM, Wageningen

2012

PhD trip 2014 Ireland

FHM, Wageningen

2014

Food Microbiology department seminars

FHM, Wageningen

TIFN expert meetings

TIFN, Wageningen 
The studies presented in this thesis were performed within the framework of TI Food and Nutrition.

Financial support from Wageningen University for printing this thesis is gratefully acknowledged.

Cover \& Layout design: AgileColor Design Studio/Atelier || AgileColor.com Printed by: Ridderprint, Ridderkerk (NL) || Ridderprint.nl 

